\title{
Turbulent Convection in an Anelastic Rotating Sphere: A Model for the Circulation on the Giant Planets
}

\author{
by \\ Yohai Kaspi \\ B.Sc., Hebrew University, 2000 \\ M.Sc., Weizmann Institute of Science, 2002 \\ Submitted in partial fulfillment of the requirements for the degree of \\ Doctor of Philosophy \\ at the \\ MASSACHUSETTS INSTITUTE OF TECHNOLOGY \\ and the \\ WOODS HOLE OCEANOGRAPHIC INSTITUTION \\ June 2008 \\ (c)2008, Yohai Kaspi. All rights reserved.
}

The author hereby grants to MIT and WHOI permission to reproduce and distribute publicly paper and electronic copies of this thesis document in whole or in part in any medium now known or hereafter created.

Author.

Joint Program in Physical Oceanography

Massachusetts Institute of Technology and Woods Hole Oceanographic Institution May 7 th 2008

Certified by.

Glenn R. Flierl

Professor

Thesis Supervisor

Accepted by

Raffaele Ferrari

Chair, Joint Committee for Physical Oceanography Massachusetts Institute of Technology and Woods Hole Oceanographic Institution 


\title{
Turbulent Convection in an Anelastic Rotating Sphere: A Model for the Circulation on the Giant Planets
}

\author{
by \\ Yohai Kaspi \\ Submitted to the Massachusetts Institute of Technology and Woods Hole \\ Oceanographic Institution on May 2008 in partial fulfillment of the requirements for \\ the degree of Doctor of Philosophy
}

\begin{abstract}
This thesis studies the dynamics of a rotating compressible gas sphere, driven by internal convection, as a model for the dynamics on the giant planets. We develop a new general circulation model for the Jovian atmosphere, based on the MITgcm dynamical core augmenting the nonhydrostatic model. The grid extends deep into the planet's interior allowing the model to compute the dynamics of a whole sphere of gas rather than a spherical shell (including the strong variations in gravity and the equation of state). Different from most previous 3D convection models, this model is anelastic rather than Boussinesq and thereby incorporates the full density variation of the planet.

We show that the density gradients caused by convection drive the system away from an isentropic and therefore barotropic state as previously assumed, leading to significant baroclinic shear. This shear is concentrated mainly in the upper levels and associated with baroclinic compressibility effects. The interior flow organizes in large cyclonically rotating columnar eddies parallel to the rotation axis, which drive upgradient angular momentum eddy fluxes, generating the observed equatorial superrotation. Heat fluxes align with the axis of rotation, contributing to the observed flat meridional emission. We show the transition from weak convection cases with symmetric spiraling columnar modes similar to those found in previous analytic linear theory, to more turbulent cases which exhibit similar, though less regular and solely cyclonic, convection columns which manifest on the surface in the form of waves embedded within the superrotation. We develop a mechanical understanding of this system and scaling laws by studying simpler configurations and the dependence on physical properties such as the rotation period, bottom boundary location and forcing structure.

These columnar cyclonic structures propagate eastward, driven by dynamics similar to that of a Rossby wave except that the restoring planetary vorticity gradient
\end{abstract}


is in the opposite direction, due to the spherical geometry in the interior. We further study these interior dynamics using a simplified barotropic annulus model, which shows that the planetary vorticity radial variation causes the eddy angular momentum flux divergence, which drives the superrotating equatorial flow. In addition we study the interaction of the interior dynamics with a stable exterior weather layer, using a quasigeostrophic two layer channel model on a beta plane, where the columnar interior is therefore represented by a negative beta effect. We find that baroclinic instability of even a weak shear can drive strong, stable multiple zonal jets. For this model we find an analytic nonlinear solution, truncated to one growing mode, that exhibits a multiple jet meridional structure, driven by the nonlinear interaction between the eddies. Finally, given the density field from our 3D convection model we derive the high order gravitational spectra of Jupiter, which is a measurable quantity for the upcoming JUNO mission to Jupiter.

Thesis Supervisor: Glenn R. Flierl

Title: Professor, Massachusetts Institute of Technology 
In memory of my father 


\section{Acknowledgments}

This thesis is dedicated to the memory of my father, Amiram Kaspi, whom I lost at a young age, but who ignited my interest in many of the things that led me to do this work.

This has been a long journey. Beyond the academics, this has been a journey of coming to a new country and becoming parents. We have lived at MIT for this whole period and often work and home became one. Above all, my biggest thanks goes to Anat, for sharing with me every step, and growing us as a family. This would not have been possible without your dedication, love and support. I thank my lovely daughters, Yuval and Hadar, for their patience with their daddy's constant work. One day "daddy's drawings" may make some sense, in the mean time as you have already discovered they make great scraps for your own creative drawings. I also thank my mother, Haya, who came here to share some time with us, and has always been a tremendous source of support and confidence.

Glenn Flierl, my advisor, has been an incredible source of inspiration. I thank Glenn for his great generosity, for spending with me all those many hours next to his blackboard, always finding new ways to approach problems, and letting me feel that things are at reach. Glenn's creativity, depth and humbleness will always be an inspiration for me. Glenn's broadness made it seem that working on the dynamics of Jupiter in an oceanographic program is ever so natural. I feel privileged to have had Glenn as my advisor.

I thank my thesis committee Joe Pedlosky, Alan Plumb and Sara Seager for their advice and suggestions over the years. I was always amazed by their ability to get so quickly and precisely to the points I was struggling with, and often they have put both Glenn and I on track when we would diverge in random directions. I thank Joe for taking me as his T.A., this has been a great privilege and one of my best experiences in graduate school.

I owe thanks to many others who have given me advice and suggestions during this work, among which are: Jean-Michel Campin, Eli Tziperman, Raffaele Ferrari, Adam Showman, Dick Lindzen, Baylor Fox-Kemper, Jonathan Lilly, Tapio Schneider, Carl Wunsch and Bill Hubbard. Particularly I thank Jean-Michel for his immense 
help with applying the MITgem to this project. I can not imagine approaching this modeling task without his close support and knowledge.

This project required significant computer resources, and I thank Eli Tziperman and Zhiming Kuang for providing me computer resources on the swell cluster at Harvard, and the aces group at MIT for providing me with computer time on their cluster.

I sat at the same desk for six years. Our office at 54-1417 was always a vibrant and enriching place to come to work at. I thank my officemates over the years, Beatriz Pena-Molino, Greg Gerbi, Holly Dail, Matt Mazloff and Stephanie Waterman for creating this atmosphere and being great friends. Specifically I thank the "aesthetics committee" of 1417 for the creative graphic advice and setting high Matlab standards. I thank my other PO classmates Ariane Verdy, Dave Sutherland and Maxim Nikurashin for their friendship and advice.

Funding was provided by the MIT Presidential Fellowship, the Charney Fellowship, WHOI Academics Programs, NSF grants OPP-9910052, OCE-0137023, AST0708106 and NASA grant NN-6066GC286. This support is gratefully acknowledged. 


\section{Contents}

1 Introduction $\quad 19$

1.1 Motivation .......................... 19

1.2 The Atmospheres of the Giant Planets of the Solar System . . . . . . 19

1.2.1 The Wind Structure . . . . . . . . . . . . . 20

1.2.2 The Thermal Structure . . . . . . . . . . . . 25

1.2 .3 Clouds ...................... 27

1.3 Previous Dynamical Models . . . . . . . . . . . . 28

1.3.1 Shallow Models ................ 28

1.3.2 Deep Models . . . . . . . . . . . . . . 31

1.3.3 Discussion: Shallow vs. Deep Approaches . . . . . . . . . . . 34

1.4 Fundamental Questions . . . . . . . . . . . . . . . 35

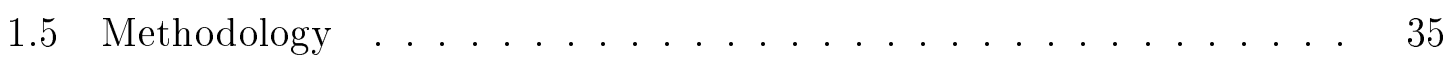

1.6 Thesis Overview . . . . . . . . . . . . . . 37

2 A Deep Anelastic General Circulation Model 41

2.1 Model Overview . . . . . . . . . . . . . . . . . . . 41

2.2 The Anelastic System . . . . . . . . . . . . . . . . . 42

2.2.1 The Anelastic Momentum Equations . . . . . . . . . . . 44

2.2.1.1 The Anelastic Equations for an Ideal Gas . . . . . 45

2.2.1.2 The Anelastic Equations for a General Equation of State 46

2.2.2 The Anelastic Thermodynamic Equation . . . . . . . . . . 48

2.2.3 Energetics of the Anelastic System with a General Equation of State ...................... 48

2.3 The Equation of State . . . . . . . . . . . . . . . 49

2.3.1 Electron Degeneracy ............... 50

2.3.2 Pressure Ionization . . . . . . . . . . . . . 53 
2.3.3 Coulomb Interactions . . . . . . . . . . . . . . . . 54

2.3.4 The SCVH Equation of State . . . . . . . . . 55

2.4 The Reference State . . . . . . . . . . . . . . . 57

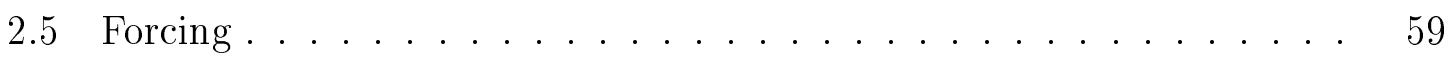

2.6 Model Summary . . . . . . . . . . . . . . . . . . . . . 61

3 Numerical Results $\quad 63$

3.1 Axisymmetric Results . . . . . . . . . . . . . . . 63

3.1.1 The Effect of Rotation on Convective Plumes . . . . . . . . 64

3.1.2 The Critical Rayleigh Number for a Rotating Fluid on a Sphere 67

3.2 From the $2 \mathrm{D}$ to the $3 \mathrm{D}$ Model $\ldots \ldots \ldots \ldots \ldots \ldots$

3.3 The 3D model . . . . . . . . . . . . . . . . . . . . . . 72

4 Basic Balances and the Vertical Wind Structure 85

4.1 Introduction . . . . . . . . . . . . . . 85

4.2 Basic Balances . . . . . . . . . . . . . . . 87

4.3 The Vertical Structure of the Zonal Velocity . . . . . . . . . . 90

4.3.1 Thermal Wind for a Deep Anelastic Setting . . . . . . . . . 90

4.3.2 The Role of Compressibility in the Baroclinic Vorticity Production ....................... 91

4.3.3 Anelastic versus Boussinesq Cases . . . . . . . . . . . . 96

4.4 The Angular Momentum Balance . . . . . . . . . . . . . 96

4.5 The Effect of Rotation . . . . . . . . . . . . . . . 100

4.6 Properties in the Zonally Asymmetric Circulation . . . . . . . . . 103

4.6.1 Formation of Columnar Convection . . . . . . . . . . . 103

4.7 Scaling Estimates for the Vertical Profile of the Zonal Wind . . . . 106

5 Weakly Nonlinear Analysis of Column Formation and Superrotation113

5.1 The Weakly Nonlinear Limit . . . . . . . . . . . . . . . . . . 114

5.2 Single Column Barotropic Model . . . . . . . . . . . . . . . 121

5.3 The Annulus Model . . . . . . . . . . . . . . . . . . . . 127

5.3 .1 Solving for the Eigenmodes $\ldots \ldots \ldots \ldots$

6 Model Sensitivity Analysis $\quad 135$

6.1 From a Spherical Shell to a Full Sphere . . . . . . . . . . . 136 
6.2 Multiple Column Layers . . . . . . . . . . . . . . . . . 140

6.3 Model Sensitivity to Nondimensional Parameters . . . . . . . . . 141

6.3.1 The Rayleigh Number . . . . . . . . . . . . . . . . . 143

6.3 .2 The Prandtl Number . . . . . . . . . . . . . . . . . . 147

6.3.3 The Ekman Number . . . . . . . . . . . . . . . . . . . 148

6.4 The Effect of the Forcing Profile . . . . . . . . . . . 150

6.5 Summary . . . . . . . . . . . . . . . . 151

7 Formation of Multiple Zonal Jets by Baroclinic Instability 153

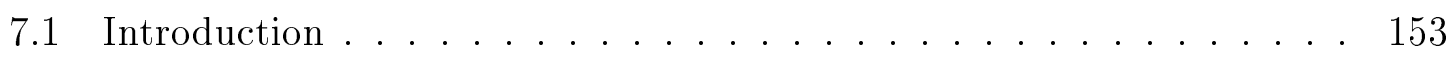

7.2 The Two-Beta Model . . . . . . . . . . . . . . . . 157

7.3 Linear Stability Analysis . . . . . . . . . . . . . . . 158

7.4 The Nonlinear Truncated Model . . . . . . . . . . . . . . . . . 161

7.5 The Weakly Supercritical Limit . . . . . . . . . . . . . 167

7.6 Fully Nonlinear Model and the Generation of Multiple Zonal Jets . . 168

7.7 Discussion . . . . . . . . . . . . . . . . . . . . . 178

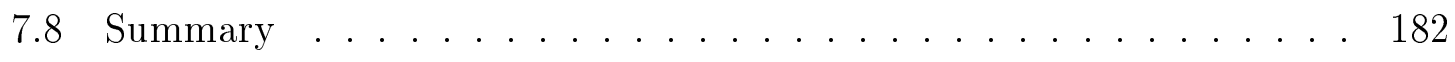

8 Conclusion $\quad 183$

8.1 Thesis Summary . . . . . . . . . . . . . . . . . . . 183

8.1.1 Convection in a Rotating Anelastic Sphere . . . . . . . . 183

8.1.2 Application to the Atmospheres of the Giant Planets . . . . 185

8.2 Future Work . . . . . . . . . . . . . . . . . . . 188

8.2 .1 Solar Forcing . . . . . . . . . . . . . . . 188

8.2 .2 Gravitational Moments . . . . . . . . . . . . . . . . 189

A Applying the Deep Anelastic System to the MITgcm 193

B Nonlinear Truncated Solutions for the Two-Beta Model 199

B.1 The Derivation of the Truncated Model . . . . . . . . . . . . . . 199

B.2 The Weakly Nonlinear Limit to the Truncated Model . . . . . . . 202

$\begin{array}{ll}\text { Bibliography } & 207\end{array}$ 


\section{List of Figures}

1.1 Mean zonal velocity as function of latitude for Jupiter and Saturn. . . 24

2.1 The Boltzmann distribution and Pauli's exclusion principle for both planetary and stellar characteristic values. . . . . . . . . 51

2.2 The effect of electron pressure on the equation of state. . . . . . . 53

2.3 The effect of pressure ionization, thermal ionization, Coulomb interactions and electron degeneracy on the equation of state. . . . . . . 56

2.4 The adiabatic reference state of the model. . . . . . . . . . . 58

2.5 Isobars in $\log \rho-\log T$ space for the SCVH EOS and an ideal gas. . . 59

2.6 The vertical heating profile of the model. . . . . . . . . . . 61

3.1 The meridional streamfunction for axisymmetric experiments with different rotation periods. . . . . . . . . . . . 65

3.2 The ratio $\frac{\Omega^{2}}{\left|N^{2}\right|}$ and the streamfunction intensity as a function of the rotation period for axisymmetric runs. . . . . . . . . 66

3.3 The critical Rayleigh number as function of latitude. . . . . . . . 69

3.4 A comparison of 2D and 3D Boussinesq and anelastic models. . . . . 71

3.5 The 1 bar surface fields averaged over 1 day for a high Rayleigh number simulation. . . . . . . . . . . . . . . 75

3.6 The 1 Mbar surface fields averaged over 1 day for a high Rayleigh number simulation. . . . . . . . . . . . 76

3.7 The 10 Mbar surface fields averaged over 1 day for a high Rayleigh number simulation. . . . . . . . . . . . . 77

3.8 Equatorial plane slices averaged over 1 day for a high Rayleigh number simulation. . . . . . . . . . . . . . . 78

3.9 Snapshots of zonally averaged fields on a meridional section for a moderate Rayleigh number simulation. . . . . . . . . . . . . 79 
3.10 Snapshots of fields at the 1 bar surface for a moderate Rayleigh number simulation. . . . . . . . . . . . . . .

3.11 Snapshots of fields on an equatorial section for a moderate Rayleigh number simulation. . . . . . . . . . . . .

3.12 The 1 bar surface fields for a run with a moderate Rayleigh number time averaged over 12 days. . . . . . . . . . . . . 82

3.13 Equatorial sections for a run with a moderate Rayleigh number time averaged over 12days . . . . . . . . . . . . 83

4.1 Meridional sections showing geostrophic balance. . . . . . . . . 88

4.2 Meridional sections showing hydrostatic balance including the vertical Coriolis component. . . . . . . . . . . . 89

4.3 The contributions of entropy and pressure to the density anomaly and

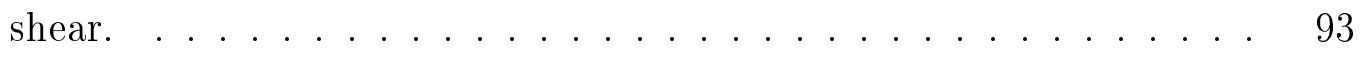

4.4 The vorticity equation balance. . . . . . . . . . . . 94

4.5 The zonally averaged zonal velocity for an Anelastic run and a Boussinesq run. . . . . . . . . . . . . . . . 95

4.6 Zonally averaged zonal velocity for Anelastic and Boussinesq runs along slices parallel to the axis of rotation. . . . . . . . . . . . 97

4.7 The radial and latitudinal contributions to the zonal momentum flux divergence. . . . . . . . . . . . . . . . 98

4.8 Angular momentum and heat (mean and eddy) fluxes in a meridional cross section. . . . . . . . . . . . . . . . . 99

4.9 Surface zonally averaged velocity components as a function of latitude. 100

4.10 The ratio $\frac{\Omega^{2}}{\left|N^{2}\right|}$ and the value of $\left|N^{2}\right|$ as a function of the rotation period.101

4.11 The nondimensional number $\chi=\left(\frac{T a \cdot P r}{R a}\right)^{\frac{1}{2}}$ as function of the rotation period and the mean zonal velocity at the surface averaged around the equator as function of the rotation period for a set of experiments with equal parameters but varying rotation period $R a=3 E 6, \operatorname{Pr}=10$. . 102

4.12 The effect of rotation: Zonally averaged velocity fields for a fast and slow rotating planet on a meridional section. . . . . . . . . . . . 104

4.13 The 2D streamfunction on radius-longitude slices oriented toward the center of the planet. . . . . . . . . . . . 105 
4.14 Velocity anomalies estimated from scaling arguments and from model results. . . . . . . . . . . . . . . . . . 107

4.15 Comparison of the scaling estimate for zonal velocity with model results for a range of Rayleigh numbers. . . . . . . . . . . . . . . . 109

4.16 Zonally averaged zonal velocity along slices parallel to the axis of rotation.111

5.1 Snapshots of the equatorial streamfunction for the weakly nonlinear run.115

5.2 The eddy momentum flux divergence and the viscous terms at statistical steady state for the weakly nonlinear run. . . . . . . . . 116

5.3 The balance between eddy momentum flux divergence and the viscous term from fluxes perpendicular to the axis of rotation. . . . . . . . 116

5.4 The evolution of the $u^{\prime}$ field in the weakly nonlinear run. . . . . . 117

5.5 The zonal mean zonal velocity on the equatorial plane in steady state for the weakly nonlinear run. . . . . . . . . . . . . . . . 119

5.6 The 2D streamfunction on conic surfaces of constant latitudinal angle for the weakly nonlinear run. . . . . . . . . . . . 120

5.7 Waves embedded in the equatorial surface zonal velocity for the weakly nonlinear and turbulent cases. . . . . . . . . . . . . . . . . 121

5.8 The phase speed of the columns for the weakly nonlinear run. . . . . 123

5.9 The effective $\beta$ as a function of radius for both Anelastic and Boussinesq cases. . . . . . . . . . . . . . . . . 125

5.10 The phase speed of the columns for the fully turbulent run. . . . . . 126

5.11 The mapping from a Cartesian grid to an annulus grid. . . . . . . 127

5.12 Propagation of vortices in the annulus model. . . . . . . . . 128

5.13 Hovmoller diagrams for cases of a positive and negative linear slope in the annulus model. . . . . . . . . . . . . . . . . 130

5.14 Eigenmodes for cases of linear and curving slope in the annulus model. 131

5.15 The angle of the spiraling of the eigenmodes for varying slopes in the annulus model. . . . . . . . . . . . . . . . . 133

6.1 The zonal mean zonal velocity as function of model depth $\ldots . . .9137$

6.2 Location of columns and meridional extent of equatorial superrotation as function of model depth . . . . . . . . . . . . . . 138

6.3 Number of columns and zonal velocity intensity as function of model depth . . . . . . . . . . . . . . . . . 139 
6.4 Solutions with multiple column layers. . . . . . . . . . . . 140

6.5 The model sensitivity to Rayleigh number. . . . . . . . . . . . . 144

6.6 The model sensitivity to the $\chi$ parameter. . . . . . . . . . 146

6.7 The model sensitivity to Prandtl number. . . . . . . . . . . . . 147

6.8 The model sensitivity to viscosity. . . . . . . . . . . . . . . 148

6.9 The model sensitivity to Ekman number. . . . . . . . . . . . . . 149

6.10 Comparison of the vertical cooling profile forcing to forcing by a bottom heat flux for the $2 \mathrm{D}$ and $3 \mathrm{D}$ cases. . . . . . . . . 150

7.1 Linear stability curves for the two $\beta$ model . . . . . . . . . . . 159

7.2 Linear growth rates in horizontal wave number space $\ldots \ldots \ldots . . .160$

7.3 The analytical baroclinic induced zonal velocity $\overline{U_{C}} \ldots \ldots$. . . . 164

7.4 Enstrophy as a function of time for the linear, truncated and nonlinear two- $\beta$ models . . . . . . . . . . . . . . 166

7.5 The induced zonal velocity meridional profile. . . . . . . . . 170

7.6 Instantaneous snapshots of total zonal velocity field for the top layer. 172

7.7 Zonally averaged fields for the top layer at steady state. . . . . . . 173

7.8 The transition from baroclinic instability to multiple jets. . . . . . . 174

7.9 The jet width as a function of the applied shear. . . . . . . . . 175

7.10 Steady state total velocity fields for runs with different applied shears. 176

7.11 The evolution of the induced zonal flow of the top layer $\overline{U_{1}}$ in time. . 177

7.12 The kinetic energy for the induced mean velocity and the eddies as a

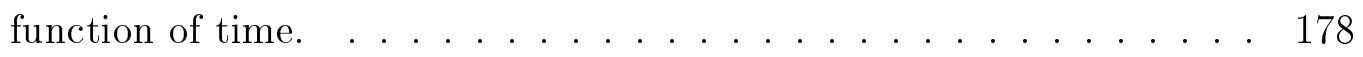

7.13 Comparison of instantaneous and time mean steady state total zonal velocity fields. . . . . . . . . . . . . . . 180

7.14 Comparison of $u_{y y}$ and $\beta$ across the channel in steady state. . . . . 181

8.1 The effect of solar forcing. . . . . . . . . . . . . . . . . . . 189

8.2 Gravitational moments for different interior velocity structures. . . . 191 


\section{List of Tables}

1.1 Properties of the giant planets of the solar system. . . . . . . . . 21

6.1 Table of parameters for numerical experiments in chapter 6 . . . . . 142

7.1 Table of numerical experiments for the two-beta model. . . . . . . . 171 


\section{Chapter 1}

\section{Introduction}

\subsection{Motivation}

The study of Geophysical Fluid dynamics (GFD) has evolved tremendously over the past 60 years. Although not complete, we have today a good basic understanding of many of the physical processes governing the dynamics of Earth's oceans and atmosphere. Many of the unresolved complexities come from the complicated interactions with continental boundaries, ice, topography, ocean bathymetry, and air-sea interactions. The giant planets which are mainly homogeneous fluid objects do not have many of these complexities and, due to their fast rotation and large scales, could be considered as "ideal" GFD objects. Yet, much of the dynamics on these objects are still poorly understood. These planets reveal some of the most striking dynamical phenomena in the solar system such as intense multiple jet streams and long-lived Earth-sized storms. Therefore studying of the dynamics of the giant planets brings opportunity for understanding how such deep atmosphere may work and gives a critical insight to our understanding of basic GFD phenomena.

\subsection{The Atmospheres of the Giant Planets of the Solar System}

The four outer planets of the solar system are mainly fluid objects. Due to the light elements constituting these planets they do not condense at solar system temperatures and therefore do not have a solid surface; rather their atmospheres are deep and merge 
smoothly with the planet's fluid interiors. Despite their size they all rotate faster than Earth, and all have latitudinal banding and high-speed jet streams. Weather patterns have a time-scale ranging from weeks to centuries, and internal heat sources, due to gravitational contraction, are big and comparable in strength to the external heating from the sun. Even Uranus, whose rotation axis is tipped in $98^{\circ}$ relative to its orbital axis, still exhibits many of the same phenomena. Here we review the characteristics obtained by observations of the atmospheres of Jupiter, Saturn, Uranus and Neptune.

\subsubsection{The Wind Structure}

Zonal Jets: The dominant feature on all the outer planets are strong zonal jets. Both Jupiter and Saturn have strong prograde eastward equatorial jets around the equator with weaker multiple east-west zonal jets away from the equator in each hemisphere. On Jupiter the equatorial superrotating region extends $17^{\circ}$ in latitude north and south of the equator (Figure 1.1), with a maximum wind speed of $140 \mathrm{~m} / \mathrm{s}$, whereas on the Saturn wind speed of the equatorial eastward jet reaches $400 \mathrm{~m} / \mathrm{s}$ near the equator, and the equatorial superrotation extends roughly $30^{\circ}$ north and south of the equator. The Jupiter superrotating equatorial jet has two peaks located $8^{\circ}$ off the equator in both hemispheres with a $30 \%$ dip in zonal velocity from maximum values at the equator itself. Wind speed measurements are made in reference to system III, a uniform rotation rate which is defined by radio emission measurements that are tied to the magnetic field which is presumably aligned with the bulk interior.

Beyond the equatorial eastward jet, Jupiter has at least six more pairs of east-west zonal jets in each hemisphere with winds with a maximum of $30-50 \mathrm{~m} / \mathrm{s}$, including one stronger jet at $24 \mathrm{~N}$ with an eastward wind reaching $130 \mathrm{~m} / \mathrm{s}$. Most jets on Jupiter have the character of a sharper eastward than westward jet, which may be a consequence of the barotropic stability limit and associated with the positive planetary vorticity gradient (see discussion in section 7.6). Until the Cassini spacecraft observations (Porco et al., 2003) it was thought that Jupiter's jets extend only up to midlatitudes, but these observations confirmed that the jets extend (though weaker than in the low latitudes), all the way to latitude $80^{\circ}$ in both hemispheres. In the high latitudes however, the zonal jets are not associated with cloud bands.

Wind velocities are deduced from cloud tracking and therefore the assumption that clouds are passive tracers of the wind is inherent to these wind measurements. 
The lack of topography and the longevity of the cloud features on Jupiter are factors that should reduce the uncertainty of these measurements. Nevertheless, if the cloud brightness or contrast is correlated to the dynamics, results might be biased (Vasavada and Showman, 2005). Observed streamlines seem not to overlap (Ingersoll, 1990) so the observed features seem to represent a single layer near the top of the clouds. Over the past 40 years of modern measurements, Jupiter's zonal wind profile has remained nearly constant. The only significant change was a decrease of $40-50 \mathrm{~m} / \mathrm{s}$ in the eastward jet near 24N (Simon, 1999). Smaller changes have been detected near the equatorial region and near the jet at $50 \mathrm{~N}$.

\begin{tabular}{|lcccc|}
\hline & Jupiter & Saturn & Uranus & Neptune \\
\hline \hline Equatorial radius $\left(10^{3} \mathrm{~km}\right)$ & 71.74 & 60.27 & 25.56 & 24.76 \\
Oblateness $\left(\%\left(R_{e}-R_{p}\right) / R_{e}\right)$ & 6.5 & 9.8 & 2.3 & 1.7 \\
Mass $\left(10^{26} \mathrm{Kg}\right)$ & 18.99 & 5.68 & 0.86 & 1.03 \\
Mean density $\left(\mathrm{Kg} \mathrm{m}^{-3}\right)$ & 1330 & 700 & 1270 & 1760 \\
Sidereal day $(\mathrm{hr}: \mathrm{min})$ & $9: 55$ & $10: 39$ & $17: 14$ & $16: 06$ \\
Solar distance $(\mathrm{AU})$ & 5.2 & 9.5 & 19.2 & 30.1 \\
Sidereal period $($ years $)$ & 11.9 & 29.5 & 84 & 165 \\
Obliquity & $3^{\circ}$ & $27^{\circ}$ & $98^{\circ}$ & $29^{\circ}$ \\
H-He fraction of mass & 99.99 & 99.8 & 98.4 & 97.9 \\
Equilibrium radiating temperature $(\mathrm{K})$ & 110 & 82 & 58 & 47 \\
Solar Flux $W \mathrm{~m}^{-2}$ & 50.66 & 14.99 & 3.71 & 1.51 \\
Emitted/absorbed flux ratio & 1.67 & 1.78 & 1.06 & 2.52 \\
Tropopause height $(\mathrm{mb})$ & 140 & 60 & 100 & 50 \\
Equatorial jet velocity $(\mathrm{m} / \mathrm{s})$ & 140 & $275-400$ & -200 & -400 \\
Number eastward jets over $10 \%$ of eq. jet & 13 & 6 & 2 & 2 \\
\hline
\end{tabular}

Table 1.1: Properties of the giant planets of the solar system (Irwin (2003), or given in text).

Saturn has a much more subdued appearance than Jupiter due to being masked by tropospheric and stratospheric haze associated with ammonia condensation. Yet, its atmosphere is even more energetic than Jupiter's. The wind structure is dominated by the wide equatorial jet which unlike the Jupiter case has gone through some significant variations between the Voyager (1981) and the Cassini (2005) areas. Voyager measurements (Ingersoll et al., 1984) have found the equatorial jet to reach $470 \mathrm{~m} / \mathrm{s}$, while more recent cloud tracking by Hubble space telescope during the period 1996-2004 showed a decrease in the intensity of the equatorial jet to $275 \mathrm{~m} / \mathrm{s}$ (Sánchez-Lavega et al., 2003). Measurements by the Cassini spacecraft in 2004 (Porco 
et al., 2005) have esstimated the equatorial jet to be between $250 \mathrm{~m} / \mathrm{s}$ to $400 \mathrm{~m} / \mathrm{s}$. A possible reason for the variation over time is the fact that the obliquity on Saturn is $26.7^{\circ}$, and therefore seasonal changes, including the significant variation in insolation due to the shadow of the rings, may have caused these changes. The high latitude jets, however, have been persistent over this period with three distinct eastward jets (center latitudes higher than $45^{\circ}$ ) in each hemisphere, all with maximum wind speed over $100 \mathrm{~m} / \mathrm{s}$ (Figure 1.1).

The ice giants Uranus and Neptune are different than Jupiter and Saturn. Their hydrogen-helium atmosphere is only a small component of their mass which is mostly composed of a large ice-rock interior. The denser ice-rock interior is estimated to occur roughly $5000 \mathrm{~km}$ below the cloud level (Irwin, 2003). Unlike the gas giants the ice giants have retrograde winds at the equator. The mean wind profiles of both planets are smoother than the gas giants with a westward broad jet at low latitudes, and an eastward jets at high latitudes. The equatorial subrotating jet on Uranus reaches $200 \mathrm{~m} / \mathrm{s}$ at the equator and spans $25^{\circ}$ degrees in latitude in each hemisphere, and the southern eastward jet peaks at $60 \mathrm{~S}$ with winds of $200 \mathrm{~m} / \mathrm{s}$ (Smith et al., 1986) as well. Because of Uranus's large obliquity and length of year, we still have not had a chance to observe its northern hemisphere with modern technology. Neptune has a stronger and wider subrotating jet reaching a zonal velocity of $400 \mathrm{~m} / \mathrm{s}$ and two high latitude jets $\left(250 \mathrm{~m} / \mathrm{s}\right.$ at $\left.70^{\circ}\right)$ (Conrath et al., 1989). The significant difference in the equatorial jet between the ice giants and gas giants may indicate a relation between the interior structure to the zonal winds. In fact in some ways it is easier to see how a retrograde jet is driven rather than a prograde jet: Hot air rising initially at rest from the interior has less angular momentum due to being closer to the rotation axis and therefore will tend to go westward at the surface. Similarly fast rotating equatorial air at the equator will acquire additional eastward momentum as it moves poleward. As we discuss in this work, a mechanism for superrotation is more complex.

Vertical wind structure: There has been only one direct measurement of the vertical structure of any of the planets. The Galileo probe descended into Jupiter's atmosphere and returned data until it reached a depth equivalent of 24 bars. The probe entered inside the equatorial jet at latitude $7.4 \mathrm{~N}$ where the eastward wind velocity at 0.4 bars was $90 \mathrm{~m} / \mathrm{s}$ (Atkinson et al., 1997, 1998) which was similar to the wind velocities previously inferred from cloud tracking (Limaye, 1986). The wind increased down to a level of 4 bars reaching $180 \mathrm{~m} / \mathrm{s}$, and then remained constant for 
as far as the data could be retrieved at the 24 bar level. This result is often used as evidence that the winds are deep, though one should remember that beyond the point that this is just a single measurement, the data only accounts for a depth of $150 \mathrm{~km}$ which are no more than $0.22 \%$ of the radius of Jupiter. In addition the probe entered in a "hot-spot" (the equatorial zone is punctuated with 10-13 such $5 \mu \mathrm{m}$ spots (Ortiz et al., 1998)), which may have anomalous dynamics because of non-zonal motions that have been associated with them (Vasavada et al., 1998).

Other than this measurement, vertical wind structure has been deduced from the horizontal temperature gradient using the thermal wind relation. This technique is very limited due to the observations being only of the higher levels. Based on thermal wind observations of air temperatures from less than 0.1 bar to $\sim 1$ bar Pirraglia et al. (1981) suggest that the jets decay with height above the cloud level (Conrath et al., 1981). Gierasch (1976) suggests that thermal contrasts arising from latent heat release during the condensation of water at altitudes of 5-10 bars can be large enough so that through thermal wind balance the jets would not extend a depth of 10 bars (Ingersoll and Cuzzi, 1969). Others suggest that due to the internal heating the atmosphere below that cloud level is close to an isentropic state and then the jets extend to the depth of the planet (Busse, 1976). We discuss these two approaches in greater length in the next section.

Recently two strong convective outbursts that erupted 9 hours apart and lasted two months were identified near the peak of the 23N jet (Sánchez-Lavega et al., 2008). They traveled at a velocity $169 \mathrm{~m} / \mathrm{s}$ which is stronger than the local jet velocity, causing significant mixing in their wake. The jet however remained robust against the turmoil generated by the disturbance evolution. This may suggest that the jet extends deeper below the upper clouds where the motions were measured.

Vortices: Besides the zonal jets the most prominent feature on Jupiter is the great red spot (GRS). The GRS is an anticyclone extending 10.5 degrees in latitude (centered at 23S) with an oval shape and a longitudinal extent of about $17000 \mathrm{~km}$ (Simon-Miller et al., 2002). The maximum velocities of the GRS range from $120 \mathrm{~m} / \mathrm{s}$ (Dowling and Ingersoll (1988), based on Voyager data) to $150 \mathrm{~m} / \mathrm{s}$ (Simon-Miller et al. (2002), based on Galileo data). The maximum relative vorticity is $6 E-5 s^{-1}$ which is roughly one third of the planetary vorticity at that latitude. The center of the vortex is found to be about $8 K$ cooler than the surrounding cloud tops. Thermal wind balance then implies that the wind speed should decrease with depth and then 


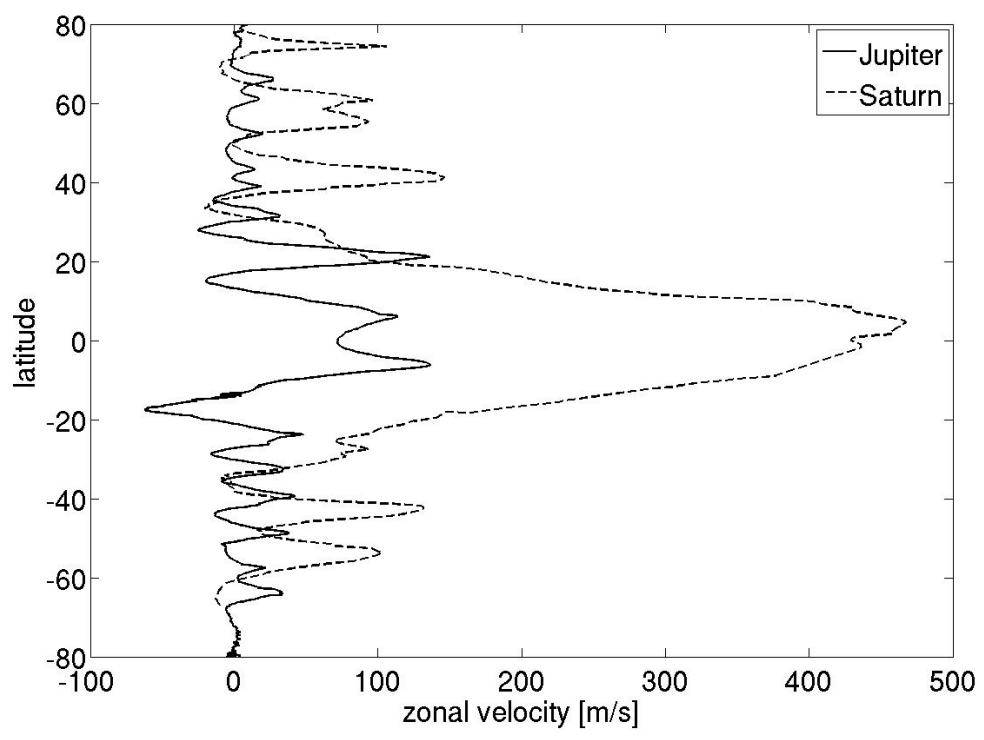

Figure 1.1: The mean zonal velocity $[\mathrm{m} / \mathrm{s}]$ as function latitude for Jupiter and Saturn as measured by Voyager. Data is courtesy of A. Sanchez-Lavega, A. Showman and A. Vasavada.

the GRS would be only $200 \mathrm{~km}$ thick. Records of the GRS go back as far as 1665 to observations made by Cassini (Cassini, 1672), indicating this anticyclonic storm has probably existed for centuries. During the period 1880-2002 the GRS has moved westward with an average speed of $3 \mathrm{~m} / \mathrm{s}$ and superimposed on this it oscillates $1^{\circ}$ in longitude every 90 days (Trigo-Rodriguez et al., 2000). There are many records (e.g. Sanchez-Lavega et al., 1998) of interactions of the GRS with other vortices absorbing part of them and expelling other parts.

Although the GRS is the largest and most sustained vortex on Jupiter, there are many other vortices with diameter ranges of $1000-5000 \mathrm{~km}$ (Simon et al., 1998). Typically the ones at high latitudes are smaller and rounder than the ones at low latitudes. The transition from round to oval vortices occurs at diameters of $\sim 2000 \mathrm{~km}$ indicating that this scale might be where the vortices feel the effect of the planetary vorticity gradient (Vasavada and Showman, 2005). In chapter 7 we use this scale as the deformation radius in the two layer model. Over $90 \%$ of the vortices on Jupiter are anticyclones. There is a broad literature on this subject and about the possible preference for anticyclones (Flierl, 1987; Marcus, 1988, 1990; Dowling and Spiegel, 1990; Yano and Flierl, 1994; Showman, 2004); in this work we do not discuss 
this issue. Interaction of vortices is often observed (Sanchez-Lavega et al., 2001), and quasigeostrophic models have been successful in describing these interactions (Youssef and Marcus, 2003).

\subsubsection{The Thermal Structure}

Temperature: Thermal infrared radiation measurements for all four giant planets show a nearly uniform meridional thermal flux profile. On Jupiter there are measurements where the poles are even found to be slightly warmer than the equator (Ingersoll, 1990), and Voyager found the poles of Uranus to be slightly colder than equator although the poles receive more sunlight (Conrath et al., 1989) due to the extreme obliquity. Radiation is emitted predominantly from the $0.3-0.5$ bar pressure level and effective temperatures are in average $124 \mathrm{~K}, 93 \mathrm{~K}, 59 \mathrm{~K}$ and $59 \mathrm{~K}$ for Jupiter, Saturn, Uranus and Neptune respectively at that level. Variations from these mean emission temperatures are mainly associated with the cloud structure and not with the latitudinal location, although solar heating is latitudinally distributed based on the season and obliquity.

All four planets have a clear tropopause at 140, 60, 100 and 50 mbar respectively (beginning with Jupiter), which have temperatures of $110 \mathrm{~K}, 80 \mathrm{~K}, 49 \mathrm{~K}$ and $50 \mathrm{~K}$ respectively (Bagenal et al., 2004). Below the tropopause the temperatures increase generally following a nearly dry adiabatic lapse rate (Lindal et al., 1981; Seiff et al., 1996). The stratospheric temperature in Saturn's atmosphere is generally lower than in Jupiter's stratosphere as can be expected due to its further distance from the sun; however, on Neptune the stratospheric temperatures are hotter than on Uranus. Only Jupiter has good exosphere measurements reaching $1350 \mathrm{~K}, 800 \mathrm{~km}$ above the $1 \mathrm{mbar}$ level (Seiff et al., 1997).

Energy balance: Measurements from both Galileo and Cassini provide estimates of the radiation at the upper atmospheres. Infrared radiation can not penetrate the clouds and therefore the measurements reflect the temperature of the upper part of the atmosphere. All planets (except Uranus) radiate away more energy than they absorb, implying an internal heat source. The radiation is also distributed more uniformly than the absorbed sun light, which suggests that there must exists some mechanism for meridional heat transport (Ingersoll and Porco, 1978). On Jupiter the emission is mostly radiated in the infrared between 10 and $100 \mu \mathrm{m}$ and has been 
estimated quite accurately. The observed energy is calculated by the incoming energy from the sun and the albedo. This allows estimating the internal flux from the core (5.444 $\pm 0.425 \mathrm{Wm}^{-2}$ on Jupiter) (Hanel et al., 1981) and calculating the energy balance defined as the ratio of emitted thermal to absorbed solar energy. On Jupiter this is $1.668 \pm 0.085$, on Saturn 1.78 (Hanel et al., 1983) and on Neptune 2.3 (Pearl and Conrath, 1991). The exception is Uranus where the internal heat flux is much less than the solar insulation and this ratio is 1.06 Pearl et al. (1990).

Thermal Waves: Several wave features have been discovered by the thermal measurements on the giant planets. Flaser and Gierasch (1986), who used Voyager images of Jupiter, discovered waves traveling within the equatorial superrotating jet with wavelengths of $300 \mathrm{~km}$. They suggested that these waves may indicate a stably stratified layer beneath the clouds supporting the propagation of gravity waves. This hypothesis was later supported by the Galileo entry probe (Seiff et al., 1997) suggesting there is a stably stratified layer between 5 and 16 bars. Similar waves were later also seen in the Galileo data, (Belton et al., 1996), and Bosak and Ingersoll (2002) suggested that these waves are produced by Kelvin-Helmholtz instabilities. A much clearer observation of these waves was recently obtained by the high resolution cameras on the New Horizons spacecraft (Reuter et al., 2007). They find the waves to persist around the planet and occupy a latitudinal region of $10^{\circ}$ around the equator. These waves have crests which extend further eastward at the equator than in higher latitudes creating crescent shaped waves propagating eastward at a phase speed of roughly double the local mean velocity. The phase speed for these waves is estimated between 204 and $276 \mathrm{~m} / \mathrm{s}$ (Reuter et al., 2007) while the local mean velocity from cloud tracking both from New Horizons and HST measurements is $100 \mathrm{~m} / \mathrm{s}$.

Larger, planetary scale waves have also been identified on Jupiter. Wavenumber 10 waves were found at equatorial latitudes at depths between 270 mbar and 1 bar by several authors (Magalhaes et al., 1989; Orton et al., 1994; Harrington et al., 1996; Deming et al., 1997). The source of the waves is unknown and hypothesis range from vertical propagation of Rossby waves (Orton et al., 1994) and mixed Rossbygravity waves (Deming et al., 1997), to connection with the plumes in the equatorial "hot-spots" (Ortiz et al., 1998; Showman and Dowling, 2000) or association with deep convective cells (Magalhaes et al., 1989). The near stationary appearance of these waves with respect to system III implies possibly a dynamical link between the interior bulk rotation of the planet (Irwin, 2003). Another interesting feature which 
was observed in stratospheric temperatures on Jupiter was a periodic 4 year variation in zonal mean temperatures at 20 mbar (Orton et al., 1991). This feature which has been continuously observed since 1978 takes the form of periodic warming of the equator and cooling of the $15^{\circ}-30^{\circ}$ latitude regions in both hemispheres, and then cooling of the equatorial region and heating of the higher latitudes. There have been attempts to link this oscillation to the QBO on Earth (Leovy et al., 1991), but the precise identification of this oscillatory behavior remains elusive.

\subsubsection{Clouds}

Jupiter's visual appearance is dominated by dark "belts" and brighter "zones" . Although the general belt/zone structure appears to be very stable, the brightness, latitudinal extent and presence of discrete features has varied significantly over time (Vasavada and Showman, 2005). The belt/zone structure is partially associated with the wind structure, where the peak of the zonal velocities appears to happen on the boundaries between the belts and zones. The zones are anticyclones, thus in the northern hemisphere they have an eastward jet on its poleward side and a westward jet on the equatorward side, and belts are cyclonic. The association between the belts/zones and wind is less clear at high latitudes. The zones appear more uniform and steadier in time than the belts, and clouds in them typically extend to higher altitudes (a few hundred mbar) than in the belts . The origin of the colors and how they respond to the winds is uncertain.

Chemical structure and Clouds: In all the outer planets the atmospheres are composed mostly of molecular hydrogen and helium, with some heavier compounds which vary between the four planets. The abundance of 'heavy' elements in the whole planet is estimated to be 3 times the solar for Jupiter, 5 times the solar for Saturn and increasing to 20-30 times solar for Uranus and Neptune. The atmospheres themselves contain only a fraction of this, and the most abundant elements after hydrogen $\left(\mathrm{H}_{2}\right)$ and helium $(\mathrm{He})$ are, in decreasing order, water $\left(\mathrm{H}_{2} \mathrm{O}\right)$, methane $\left(\mathrm{CH}_{4}\right)$, ammonia $\left(\mathrm{NH}_{3}\right)$, and hydrogen sulphide $\left(\mathrm{H}_{2} \mathrm{~S}\right)$ (Irwin, 2003). The upper atmospheres are cold enough so that some of these elements condense at various levels forming the observable cloud decks. On Jupiter the visible clouds are usually ammonia colored by sulfur, phosphorus and carbon compounds, and their top pressures are thought to be in the range of 0.3 to 3 bars while their base at 5 to 15 bars. 


\subsection{Previous Dynamical Models}

Two general approaches have been taken to explain the strong zonal jets on the Jovian atmospheres. The two emerged almost at the same time in the mid 1970's after the first detailed observations were obtained by the first space missions. Busse (1976), inspired by the Taylor-Proudman effect, suggested that if the flow is deep and extends all the way through the planet, then the jets may be the surface manifestation of differentially rotating cylinders concentric with the planet's rotating axis. On the other hand, geostrophic turbulence theory (Rhines, 1975, 1979), assuming the dynamics are confined to an outer "weather layer", suggests that the zonal jets emerge from decaying or stochastically forced turbulence on a $\beta$ plane. These two approaches have been in debate ever since.

\subsubsection{Shallow Models}

The first to apply the "shallow" approach to Jupiter was Williams $(1978,1979)$ who used both barotropic and baroclinic models to show that an imposed turbulent eddy field can lead to an inverse energy cascade leading to jets on the order of the Rhines scale. Other authors have studied zonal jets appearing from geostrophic turbulence (Vallis and Maltrud, 1993; Cho and Polvani, 1996; Huang and Robinson, 1998; Manifori and Young, 1999; Huang et al., 2001; Smith, 2003; Lee, 2004). Panetta (1993) showed that jets can emerge from baroclinic instability in a two layer model which has an imposed thermal gradient. This model allows transfer of energy from the upper to the lower layer and results in an equivalent barotropic jet. These jets seem persistent and stable, however they appear primarily when averaged, while the instantaneous fields are dominated by the eddies. Williams (2003) has produced jets in a baroclinic primitive equation system on a sphere and shows that, depending on details of the stratification and shear, the jets can migrate equatorward. Cho and Polvani (1996) impose an eddy field in a shallow water layer on a sphere and show that the eddy field evolves to a set of zonal jets at the lower latitudes, with an equatorial westward jet. Using a barotropic vorticity model with small scale random forcing and large scale friction Huang et al. (2001) and Galperin et al. (2001) suggest a scaling law to the energy spectra of the jets and show (Galperin et al., 2001, 2006) that it matches the spectrum of the observed jets on Jupiter. Smith (2003) shows multiple jets emerging

from stochastically forced QG turbulence in an equivalent barotropic system. Show- 
man (2007) show that shallow water simulations forced by mass pulses representing episodic thunderstorms in the Jovian atmosphere (Ingersoll and Cuong, 1981) can form equatorial jets (subrotating) and anticyclonic vortices at higher latitudes.

As applied to a gas giant's atmosphere, these shallow water or quasi-geostrophic models have several flaws exemplified by comparison to Jupiter: first, the observed winds violate the barotropic stability condition (Ingersoll and Cuong, 1981), thus $\beta-u_{y y}<0$ at some latitudes, although the zonal winds appear to be very stable. In contrast, all of the models produce curvatures $u_{y y}$ which are smaller than $\beta$, so that the predicted jets are weaker or wider than the Jovian ones. Second, none of these models can reproduce a superrotating jet at the equator. Some shallow water models (Cho and Polvani, 1996; Cho and Polvani, 1996; Iacono et al., 1999a,b) produce a westward retrograde jet, and typically the jets that are produced are not much stronger than the eddy field. Third, these shallow models assume a boundary at a depth of about one scale height, with the fluid below being motionless. But the thermal wind shear observed on Jupiter (Conrath et al., 1981; Gierasch et al., 1986) suggests that the flows will extend deeper and may increase, rather than die out, with depth. The Galileo probe showed this kind of velocity structure (Atkinson et al., 1996), implying two separate regimes; an upper radiative regime (above 4 bars) and an inner deep adiabatic regime below. Fourth, these models either require random forcing or deal with decay of strong initial perturbations, leaving it unclear how such a state can be maintained. The exceptions, Panetta's (1993) and Williams' (2003) baroclinic instability models, require large-scale baroclinicity strong enough to satisfy the Charney-Stern theorem, so that turbulence can be generated and maintained by feeding on the available potential energy. But the observed global scale temperature differences (Ingersoll, 1976; Hanel et al., 1981, 1983) seem to be much smaller. Finally, for Jupiter, Saturn and Neptune the internal heat flux is estimated to be as strong as the absorbed heat flux from the sun (Hanel et al., 1981, 1983; Pearl and Conrath, 1991); the shallow models do not attempt to account for the heat balance.

In Kaspi and Flierl (2007) (also chapter 7 of this thesis) we show that baroclinic instability in a two layer quasigeostrophic model with the bottom layer having a different planetary vorticity gradient representing the deep convective columns (see next section), can form multiple zonal jets that appear in the instantaneous fields (thus stronger than the eddies), and violate the barotropic stability condition but still are stable and consistent in time. Unlike the previous baroclinic models (Panetta, 
1993; Williams, 2003) due to the different geometry this model does not require a high level of baroclinicity, to generate turbulence which then cascades to zonal jets.

Another approach using a shallow model was to try and deduce the deep circulation by observing the potential vorticity in the overlying flow. Dowling and Ingersoll (1988, 1989) have derived a family of possible equivalent height fields by assuming conservation of potential vorticity in a barotropic shallow layer. This allows deducing the deep flow from the data (up to a parameter), without making apriori assumptions about the deep layer. One problem with this approach is that the only place where there is enough variation in vorticity is near big vortices such as around the giant red spot and white oval. Dowling (1993) shows that this family of equivalent height fields, corresponds to a case where the deformation radius is on the order of the Rhines scale, and then the flow is stable. Further, by later observations from the impact of comet Shoemaker-Levy on Jupiter (Hammel et al., 1995), a specific member of this family can be singled out (Dowling, 1995), and a prediction can be made about the strength of the deep flow which is comparable to the value obtained from the Galileo entry probe (Atkinson et al., 1996).

Ioannou and Lindzen (1993a,b, 1994) put forward a totally different approach to explain the zonal jets (Lindzen, 1991). They suggest that if the interior is even marginally statically stable, then tides from a dominant moon may provide the momentum source maintaining the jets. They show that the response to the tides results in high order Hough modes, which have meridional alternations resembling the alternations in the jets.

For Earth's atmosphere shallow water and quasi geostrophic models have had tremendous success in describing some of the fundamental dynamics. Due to the differences in the Jovian atmosphere pointed above it is not clear if this would be the case for the giant planets. Yet, the striking similarity of some of the phenomena observed on the Jovian atmosphere to the terrestrial atmosphere, and to features obtained in these models would lead to think that at least part of the dynamical understanding is captured by the shallow models. Showman et al. (2006) point out that the source of the forcing (whether deep or shallow) may be decoupled from whether the zonal winds are deep or shallow. Therefore even if the winds are deep they might have shallow sources and visa versa. Next we turn to discus the second approach - the deep models. 


\subsubsection{Deep Models}

The "deep" approach assumes that the jet's generation comes from within the interior of the planet. The assumption is based on the fact that since the planet is heated from within, convection drives it close to an adiabatic state, with nearly zero stratification, leading to Taylor columns that penetrate throughout the planet, and therefore there is no confinement to a thin spherical shell. Inspired by laboratory experiments (Busse, 1970), where a homogeneous rapidly rotating sphere was heated from the inside and such a multi-column structure was formed, Busse $(1976,1994)$ suggested that the interior of a planet may be occupied by Taylor columns that surround a hot core. He suggested that the multi-layered structure of convection rolls might produce the zonal jets through nonlinear interactions among the columns.

The problem of onset of convection in a rotating sphere was first studied in terms of axisymmetric solutions (e.g. Chandrasekhar, 1952), but as noted first by Veronis (1959) convection tends to form non-axisymmetric cells. Chandrasekhar (1961) set the standard formulation for the rotation dominated problem which was adopted in following work discussed here. Roberts (1968) showed that for large enough Taylor numbers (rapid rotation) the asymmetric modes will be the fastest growing unstable modes. These modes also appeared in laboratory experiments (Busse, 1970) and were the basis for Busse's model for Taylor columns in the interior of the giant planets (Busse, 1976). In both cases the asymmetric modes where confined to a thin chain of convection columns at a distance of about half the radius from the axis of rotation. Later studies (Zhang and Busse, 1987) showed that the radial structures of these modes are sensitive to the Prandtl number with a sharp transition between two distinct modes. In the first mode, where Prandtl numbers are higher, convection columns are at about half the distance to the axis of rotation as suggested by the asymptotic theory (Roberts, 1968). However, as the Prandtl number is decreased, the columns begin to stretch and develop a spiraling shape (Zhang, 1992). Decreasing the Prandtl number beyond a critical point leads to a new state with circular modes attached to the outer wall. Zhang (1994) showed that these modes can be understood as inertial oscillations which are slightly modified by the effects of viscosity and buoyancy. These calculations matched asymptotic theories discussing the radial dependence of the unstable modes for the linear problem of the onset of convection,

and was studied both in equivalent cylindrical systems (Yano, 1992) and for a full sphere (Jones et al., 2000). 
Ingersoll and Pollard (1982) noted that a columnar structure as described by Busse in a sphere is analogous to $\beta$ plane dynamics only with a different definition of $\beta$. Consequently arguments from Rhines (1975) may still apply and deep twodimensional turbulence may create jets. An advantage of this theory is that the Ingersoll and Pollard (1982) equivalent barotropic stability criterion, which has an effective $\beta$ which is negative and three times the value from the standard planetary $\beta$, is more consistent with the Jupiter data. On Jupiter the observed winds are close to marginal stability according to this criterion. Yano and Flierl (1994) have used this idea of a negative bottom layer $\beta$ to demonstrate its effect on an isolated vortex like Jupiter's giant red spot in a zonal jet. We use this parametrization for the bottom layer in the two layer model in chapter 7.

The spiraling modes obtained by Zhang (1992) have a structure that adjacent convection cells have opposite circulations. This character for weak linear convection appears in other studies as well (e.g. Zhang and Schubert, 1997; Christensen, 2002). Following the negative $\beta$ plane idea of Ingersoll and Pollard (1982), such a structure when perturbed, develops local relative vorticity based on the interaction of the column with an exterior boundary, as the columns conserve their total circulation when stretched or squeezed (Busse, 1994). Such an interaction can cause propagation of the vortices similar to a propagation of a Rossby wave (Busse, 1986). Busse and Hood (1982) showed that linear modes will tend to tilt based on the direction of the outer boundary slope, and eastward or westward shear will form. This shear however was no stronger than the perturbation itself. The spiraling alternating linear modes obtained by Zhang (1992) have positive Reynolds stresses which can create a mean flow. Zhang and Schubert $(1996,1997)$ have showed that even for a thermally driven convective interior bounded by a corotating convectively stable stratified layer, the fluid motions resulting from the instability develop similar linear modes that concentrate primarily in the outer stable region.

All the models discussed above were limited to either linear or weakly nonlinear regimes. It is not obvious that any of these modes, and therefore the resulting mechanisms can be maintained in the nonlinear regime. Glatzmaier and Olson (1993) showed numerically Taylor columns can still be maintained when the Rayleigh number is 50 times critical but their experiment was limited to a slowly rotating regime. A second shortcoming of the models discussed above, is that they were all limited to the Boussinesq approximation. The only compressible attempt to model such 
flows (Gilman and Glatzmaier, 1981) was in the solar context (slow rotation) using the anelastic approximation for an ideal gas (Ogura and Phillips, 1962), where they showed that non-axisymmetric convection modes still exist in the compressible fluid for the parameter region of their examination.

With the advance of computational abilities numerical 3D models (Sun et al., 1993; Aurnou and Olson, 2001; Christensen, 2002) solving the full Navier-Stokes equations subject to the Boussinesq approximation have demonstrated that in a rapidly rotating system a broad eastward flow can develop at the equator. This flow has been referred to result from the so-called Busse columns, though none of these studies actually demonstrated such columns explicitly. Christensen (2002) shows formation of spiraling convection cells in a 3D numerical model for case of quasi-stationary convection and shows that for higher Rayleigh numbers the convection becomes chaotic with a superrotating equatorial flow and higher latitude subrotating flow. The subrotating flow had near equal velocity along the direction of the axis of rotation. A major difference between these flows and the one suggested by Busse (1976) is that they did not develop multiple nested cylinders that would interact and produce multiple zonal jets. Multiple band structures which result from columnar convection have been shown in laboratory experiments by Manneville and Olson (1996) though these bands occupy region only within $45^{\circ}$ from the equator. Heimpel et al. (2005) using a Boussinesq model covering one tenth the depth of the planet and a longitudinal section of $45^{\circ}$, have produced high latitude jets driven by internal convection which appear when time averaged. These jets though seem to depend on the bottom flux fed by the Rayleigh-Benard type convection, and the width of the equatorial flow depends on the location of the bottom boundary.

The biggest objection to the deep theories is that we do not observe any definite columnar features at the top levels and the similarity between north and south hemisphere, although partially apparent, is not exact. This though can be resolved by the fact that at the cloud levels other processes including 2D turbulence can play a role breaking the symmetry at that level. Another criticism of the deep models is that they do not take into account the existence of a magnetic field (Kirk and Stevenson, 1987). This is based on the notion that the transition between molecular and metallic hydrogen acts as an interface and inhibits the convection from acting across that interface (Stevenson and Salpeter, 1976). The depth of that transition remains poorly known but probably lies between $0.7-0.9$ Jupiter radii and at pressures of 
1 - 3 Mbar (Guillot et al., 2004). Lorentz forces in the metallic region can act to break the zonal flows there. Recently, Liu (2006) suggested that if the zonal flows were completely barotropic and magnetic field in the interior can be inferred from their exterior values, then ohmic dissipation will cause breakdown of the deep zonal flow even above the level of the phase transition. Laboratory work indicates that the transition from molecular to metallic hydrogen may not be sharp in density and conductivity (Weir et al., 1996).

The two major drawbacks of these models are the use of the Boussinesq approximation and having the physical understanding of the dynamics limited to the linear models. In this work we attempt to address these two issues.

\subsubsection{Discussion: Shallow vs. Deep Approaches}

Both approaches have compelling arguments to why they are important to the dynamics. On one hand due to the strong convection it is hard to escape having a nearly barotropic interior and then the Taylor-Proudman theorem will hold in the interior. On the other hand the resemblance to terrestrial weather and the fact that infrared observations show that the atmosphere is not barotropic near the cloud level, supports the approach that there is a stability stratified baroclinic level beneath the clouds and the dynamics may be governed by shallow processes only. Bridging the two approaches, a scenario that the atmosphere is indeed barotropic beyond some level but the velocities have become weak by that depth would be therefore be a plausible case. However, the Galileo probe which showed that indeed the atmosphere is baroclinic but in the "wrong" way; therefore increasing velocities down to a certain depth where they become constant would seem to lead back to the importance of deep processes.

An important difference worth noting between the shallow and deep approaches, is that the shallow models assume that only full 2D turbulence can explain the jets, while deep models suggest that stepping up from linear to weakly nonlinear theory leads to closer understanding of reality. Obviously linear dynamics could not describe the mechanisms leading to formation of jets in 2D turbulence; however as we show in chapter 7 weakly nonlinear baroclinic instability can give insight to the formation of quasigeostrophic jets. On the other end we show the transition from weakly nonlinear to fully turbulent dynamics in our deep model. 
An appealing possibility is that the actual jet structure lies somewhere between the two, shallow verses deep, scenarios. Ingersoll and Cuong (1981) argue that the zonal flow is deep rooted while the coherent vortices like the GRS are confined to the shallow part of the atmosphere. However, Yano and Flierl (1994) point out that a baroclinic GRS produces a barotropic radiating field and thus the GRS could not be sustained. Vasavada and Showman (2005) point out that such a deep rooted superrotation underlying a shallow atmosphere can explain the near, but imperfect, symmetry between northern and southern hemispheres. In this respect as pointed by Yano (1994) the coupling of deep thermal convection with the atmospheric circulation is the next step for modeling.

\subsection{Fundamental Questions}

The previous two sections have pointed to the key observational data and modeling approaches in our attempt to understand the dynamics on the giant planets. Above all they indicate the discrepancy between the amount of data that we know and the level of understanding we have about the dynamics. Questions such as, what drives the zonal jets? what controls the speed and width of the zonal jets? Why are the equatorial jets on the gas giant superrotating? Why is there an opposite equatorial rotation on the ice giants? How deep are the zonal jets? What controls the jets stability? What drives the wave features observed within the equatorial superrotation? and what causes the uniform emitted thermal flux, are all first order questions that must be answered to understand these dynamics. Our goal is to try and address all these questions, and we come back to discuss them in chapter 8 .

\subsection{Methodology}

The previous sections highlighted the need for a model which is both non-Boussinesq and capable of studying convective turbulence in the full 3D system. Our main tool in this thesis is such a model that we built based on the non-hydrostatic dynamical core of the MITgcm. We focus on the understanding of specific physical processes using simplified configurations of this model, a variety of other simpler numerical models and analytic models. Our new general circulation model is an improvement over previous models in several aspects: It is both non-hydrostatic and non-Boussinesq 
and thus can address convection in a compressible system (anelastic). The model is deep, and therefore can address a full sphere of gas (beside a small interior core), with a constant number of vertical levels per scale height, thus keeping a high resolution in the atmosphere. It uses an equation of state suited for hydrogen-helium mixtures and therefore beyond accounting for the compressibility it has the capability of including the complex thermodynamics in the deep interior of the planet. Finally, it uses a forcing scheme that represents the cooling of the whole vertical structure, different from Rayleigh-Benard type convection set by the boundaries, and has a radially dependent gravity field and thermodynamic variables.

We progressively build a physical understanding of the dynamics beginning from the simpler 2D slowly rotating and Boussinesq cases and move to the 3D rapidly rotating and anelastic cases. We perform studies for understanding the roles of processes such as rotation and stratification. For the full 3D anelastic model we extend these process studies to explore the parameter space of Rayleigh, Ekman and Prantdl numbers and other model settings such as the total aspect ratio and forcing. We then focus on the mechanisms driving the cyclonic convection columns, baroclinic shear and equatorial superrotation. We show that the mechanisms suggested in previous work of deep convection models mostly in the linear and weakly nonlinear regimes can be identified in the GCM for the weakly turbulent cases. The transition to stronger turbulent regimes possesses some of the same mechanisms but also has some differences.

Since Jupiter is the giant planet that we have the most data about both in terms of meteorology and internal thermodynamics, we set our model parameters to the Jupiter regime. Many of the physical processes that we find however would be applicable to Saturn as well. There is a high level of uncertainty regarding dynamics in the plasma interior of the planet. Most previous models set the bottom limit above or at the level of the molecular-metallic boundary. Although this might not be the best representation of Jupiter itself, we deliberately push the bottom limit well below this level in effort to study the dynamics when the vertical and horizontal scales are comparable. In fact, as we show, when using a thinner (and maybe more realistic) spherical shell some of the dynamical features, such as the width of the superrotating jet, resemble more the observations of Jupiter. In order however not to be biased by this, and for the generality of the study most of the analysis is done with an aspect ratio factor of two between outer and inner shell boundary. We do however show the 
whole range from a thin spherical shell to a full sphere.

A completely different model is used in chapter 7 to study the formation of multiple zonal jets. This is a quasigeostrophic two layer model, which has a representation of the deep dynamics which are demonstrated by the full GCM (although this study proceeded the development of the GCM). Here again we use a hierarchy of models ranging from linear stability analysis, through a weakly nonlinear theory and a nonlinear model truncated to one growing mode, to a fully nonlinear model. We show that multiple zonal jets can form from baroclinic instability and an inverse energy cascade in geostrophic turbulence.

\subsection{Thesis Overview}

We begin in chapter 2 by a description of the new general circulation model. Beyond the issues of adapting the MITgcm dynamical core to the deep anelastic system, in this chapter we discuss in detail the anelastic approximation itself and present a generalization to previous work showing that the anelastic approximation can be applied, and is energetically consistent, with a general equation of state. Chapter 3 discusses results from the numerical model, beginning from results from 2D axisymmetric calculations through results from the 3D anelastic calculations. Within the 2D framework we present only results that are robust and hold for the 3D case (such as the effect of rotation), or results which are different (such as equatorial zonal flows) but highlight the role of the asymmetries in driving the 3D dynamics. Another result obtained from the axisymmetric model is the dependence of the critical Rayleigh number on latitude. We solve for the 2D Boussinesq case using a local approximation analytically, and then demonstrate numerically. The latter part of this chapter is devoted to presenting results from the 3D anelastic model which will be a framework for future discussion and interpretation.

In chapter 4 we discuss the baroclinic structure of the zonal velocity. The main paradigm here is that the Taylor Proudman theorem should apply for the zonal velocity whether the fluid is anelastic or Boussinesq as long as the fluid is in a barotropic state. We show that baroclinic contributions due to convection are in fact important in driving the velocity away from the Taylor-Proudman regime, and the baroclinic contributions due to compressibility create a shear in the zonal velocity while keeping the alignment with the axis of rotation. We show that although the absolute value 
of the velocities depends on the model parameters the vertical structure of the zonal flow does not. We look at the zonally averaged momentum budget, and show that eddy momentum fluxes acting away from the axis of rotation drive momentum to the exterior to generate the superrotating equatorial winds. These eddy momentum fluxes are strongest along a cylindrical surface within the sphere. We show that this cylindrical surface is caused by smaller scale convection cells, parallel to the axis of rotation, which surround the interior core and penetrate throughout the planet. Different from the convection columns suggested by Busse (1976), these cells are all cyclonic.

In chapter 5 we use three different models to focus on the mechanisms driving the cyclonic columns and equatorial superrotation. We look at the GCM in a regime of weak convection where we can better identify the physics driving the circulation we see in the more turbulent cases. This parameter regime of the GCM allows us to clearly identify the positive (eastward) phase speed of the convection columns. It shows the transition from an initial weak-velocity state with alternating cyclonic and anticyclonic modes, which are similar to modes seen in linear and weakly nonlinear studies such as Zhang (1992); Zhang and Schubert (1997), to a state with only cyclonically rotating columns. The correlation within the columns between the zonal and vertical velocity anomalies drives the upgradient angular momentum fluxes. This weakly nonlinear mode of the model also allows us to follow in a more precise way (due to the less noisy solution) the momentum budget. We follow Ingersoll and Pollard (1982) and show that their barotropic cylindrical model represents well some aspects of the turbulent interior and can explain the direction of propagation (through an equivalent Rossby wave mechanism) and roughly account for the number of convection columns. Finally, we focus on the mechanism for the angular momentum flux by using a simplified barotropic annulus model which allows studying the zonal tilt in the eigenmodes, which are analogous to a slice through the spiraling convection columns seen in the full GCM, and point to the role of the planetary vorticity gradient and viscosity in creating these modes.

In chapter 6 we explore the parameter space of the model. Due to the relative simplicity of the model the parameter space is rather limited and allows doing a sensitivity analysis to most parameters. We divide the parameters into two groups: one of parameters which are associated with the specific configuration of the model such as the location of the boundaries and model resolution; and the second are 
parameters controlling the coupled six equations we solve which are the Rayleigh, Ekman, Prandtl numbers, the choice of the forcing profile and the details of the equation of state. We begin by varying the depth of the domain from a thin shell (10\% of the planet's radius) to almost a full sphere (93\% of the radius) and look at the implications in terms of the location of the columns and details of the zonal flow. Then we do a systematic study varying the nondimensional numbers controlling the simulations, look at specific solutions which appear during spin-up and study the dependence on different forcing profiles. Since this is a new model this study is essential for any interpretation of our results.

Chapter 7 stands on its own as an independent study, but uses some of the concepts developed in the previous chapters as motivation for the model setup. The main concept we take from the deep model (and was suggested originally by Ingersoll and Pollard, 1982) is a negative $\beta$ plane which comes from the opposite direction of the background planetary vorticity gradient in the interior of a fluid sphere demonstrated in chapter 5. We propose that baroclinic instability of a weak shear may play an important role in the generation and stability of the strong multiple zonal jets observed in the atmospheres of the giant planets. We use a two-layer quasigeostrophic model on a $\beta$ plane where the bottom layer has a negative $\beta$. Linear stability theory predicts that the high wave number perturbations will be the dominant unstable modes for a small vertical wind shear like that inferred from observations. We develop a nonlinear model truncated to one growing mode which generates a multiple jet meridional structure, driven by the nonlinear interaction between the eddies. In the weakly supercritical limit, this model agrees with previous weakly nonlinear theory, but it can be explored beyond this limit allowing the multiple jet induced zonal flow to be stronger than the eddy field. Calculations with a fully nonlinear pseudo-spectral model produce stable meridional multi-jet structures when beginning from a random potential vorticity perturbation field. The instability removes energy from the mean state weak baroclinic shear and generates turbulent eddies that undergo an inverse energy cascade and form multi-jet zonal winds. The jets are the dominant feature in the instantaneous upper layer flow, with the eddies being relatively weak. The jets scale with the Rhines' length, but are strong enough to violate the barotropic stability criterion. We show that the basic physical mechanism for the generation and stability of the jets in the fully nonlinear two layer numerical model is similar to that of the truncated model. 
The model discussed in chapter 7 points out the possible importance of the interaction between the convectively driven interior and the shallow stably stratified atmosphere. In chapter 8 we discuss preliminary results of such coupling using the anelastic GCM when driven by both convection and solar forcing. We show a possible application for our anelastic model for the upcoming JUNO mission to Jupiter (2011) which will measure the high order gravity moments. We follow on a suggestion by Hubbard (1999) that precise measurements of the high order gravitational moments can give information on the deep wind structure of the planet. We calculate the gravitational moments resulting from the density field for different end-state velocity profiles. In chapter 8 we conclude and summarize our results both from the pure fluid mechanical aspect of the problem, and the application to the dynamics and circulation on the giant planets. 


\section{Chapter 2}

\section{A Deep Anelastic General Circulation Model}

\subsection{Model Overview}

We are interested in studying the dynamics of system where the fluid is not confined to a spherical shell, is driven by internal convection and the density varies over several orders of magnitude. Previous attempts to model this system fall into two general categories: convection models with deep geometry that are limited to Boussinesq dynamics (e.g. Zhang and Schubert, 1997; Aurnou and Olson, 2001; Christensen, 2002; Heimpel et al., 2005), or spherical shell atmospheric type models which lack or parametrize the interior convection (e.g. Cho and Polvani, 1996; Lee, 2004; Lian et al., 2006). The idea of forming such a model is two fold: one reason is to address in a new way some of the questions presented in chapter 1 regarding the dynamics on the giant planets. The second reason is to look at new aspects of fluid dynamics of a rotating sphere in which the gravity and rotation vectors are not parallel. Such analysis has never been attempted in a system which is non-Boussinesq, non-hydrostatic and has a realistic equation of state which is dependent on the pressure variations. As we will show in the next chapter this model also allows us to reach more turbulent regimes than achieved in previous work.

A main complexity of this problem is that the system varies in more than four orders of magnitude in density (from about a tenth the density of air at 1 bar to a few times the density of water at $10 \mathrm{Mbar}$ ), and therefore requires accounting for 
the compressibility of the gas. Typically this problem is overcome by using pressure coordinates which allows us to use equivalent Boussinesq dynamics, with redefining the vertical velocity, and still to account for the compressibility of the gas (Vallis, 2006). However since this is a convective system and we need to conserve all nonhydrostatic components in the momentum equations, the use of pressure coordinates brings additional difficulties. Therefore we have constructed the model in regular depth coordinates, but use the anelastic approximation to account for the variations in density. This approximation allows for the variations in mean density but neglects the density anomalies in the mass equation. Although a natural starting point for this model would seem to be an atmospheric model, the flexibility, the available nonhydrostatic core, the reliability, and the available support at MIT led us to choose to use the MITgcm.

\subsection{The Anelastic System}

The anelastic approximation was first introduced by Batchelor (1953) for a adiabatically stratified horizontally uniform reference state. Then it was more rigorously presented by Ogura and Phillips (1962) in order to filter sound waves in a nonhydrostatic system. In essence, they perform a linearization around a specified adiabatic state $\bar{s}=s_{0}$ which defines a reference pressure $\bar{p}(r)$ and density $\bar{\rho}(r)$. The mass equation loses the $\frac{\partial \rho}{\partial t}$ term (thereby eliminating the fast sound waves); Ogura and Phillips showed that with suitable changes in other equations and using an ideal gas, the anelastic system conserves energy. Durran (1989) showed a more general solution which he called the pseudo-incompressible approximation, where he relaxes the assumption that entropy anomalies are small compared to the reference adiabatic state. In the pseudo-incompressible system density fluctuations which arise through fluctuations in pressure are neglected, and density fluctuations from temperature are figured into the mass balance. Durran's solution may be better applicable for systems with large horizontal temperature variations, however in a convective system with a large range of densities and pressures, one can not assume density fluctuations due to pressure are small, while due to the convection the reference state may be close to adiabatic. Both Ogura and Phillips and Durran assume the fluid is an ideal gas, while for the interior of the giant planets the gas diverges significantly from an ideal gas (section 2.3). We have extended the derivation for a general equation of state, 
and demonstrated that as long as the mean state is close to adiabatic the system will conserve energy; this is shown in section 2.2.3. Taking the density and pressure to be

$$
\begin{aligned}
& \rho \cong \bar{\rho}(r)+\rho^{\prime}(\phi, \theta, r, t) \\
& p \cong \bar{p}(r)+p^{\prime}(\phi, \theta, r, t)
\end{aligned}
$$

defines a background hydrostatic state

$$
\frac{d \bar{p}}{d r}=-\bar{\rho} g(r)
$$

where the gravitational acceleration $g(r)$ is also a function of depth and is defined by

$$
g(r)=\int_{0}^{r} \frac{G \bar{\rho}\left(r^{\prime}\right)}{r^{\prime 2}} d r^{\prime}
$$

where $G$ is the Cavendish constant. The density and pressure anomalies vary both spatially and temporally. With the anelastic approximation the continuity equation therefore takes the form

$$
\nabla \cdot(\bar{\rho} \mathbf{u})=0
$$

where $\mathbf{u}$ is the $3 \mathrm{D}$ velocity vector. Throughout the thesis we will try and keep the equations concise using vector form, but in this section, for completeness, we will write the model equations in the full form. Given the spherical nature of the problem we will use spherical coordinates, where $\phi$ is the longitude, $\theta$ is the latitude and $r$ is the radial coordinate. Therefore the velocity vector is defined in spherical coordinates as

$$
(u, v, w) \equiv\left(r \cos \theta \frac{D \phi}{D t}, r \frac{D \theta}{D t}, \frac{D r}{D t}\right)
$$

With $\bar{\rho}$ defined by (2.1) and with the divergence operator in spherical coordinates, the mass equation (2.5) takes the form

$$
\frac{\bar{\rho}}{r \cos \theta} \frac{\partial u}{\partial \phi}+\frac{\bar{\rho}}{r \cos \theta} \frac{\partial}{\partial \theta}(v \cos \theta)+\frac{1}{r^{2}} \frac{\partial}{\partial r}\left(\bar{\rho} r^{2} w\right)=0
$$




\subsubsection{The Anelastic Momentum Equations}

The momentum equations for a rotating fluid when applying the anelastic approximation, thus assuming $\rho^{\prime}(\phi, \theta, r, t) \ll \bar{\rho}(r)$, in spherical coordinates become

$$
\begin{aligned}
\frac{D u}{D t}+\frac{u w}{r}-\frac{u v}{r} \tan \theta-2 \Omega \sin \theta v+2 \Omega \cos \theta w & =-\frac{1}{\bar{\rho} r \cos \theta} \frac{\partial p^{\prime}}{\partial \phi}+\nu \nabla^{2} u \\
\frac{D v}{D t}+\frac{w v}{r}+\frac{u^{2}}{r} \tan \theta+2 \Omega \sin \theta u & =-\frac{1}{\bar{\rho} r} \frac{\partial p^{\prime}}{\partial \theta}+\nu \nabla^{2} v \\
\frac{D w}{D t}-\frac{u^{2}+v^{2}}{r}-2 \Omega \cos \theta u & =-\frac{1}{\bar{\rho}} \frac{\partial p^{\prime}}{\partial r}-\frac{\rho^{\prime}}{\bar{\rho}} g+\nu \nabla^{2} w
\end{aligned}
$$

where $\frac{D}{D t}$ is the material derivative,

$$
\frac{D}{D t}=\frac{\partial}{\partial t}+\frac{u}{r \cos \theta} \frac{\partial}{\partial \phi}+\frac{v}{r} \frac{\partial}{\partial \theta}+w \frac{\partial}{\partial r} .
$$

$\Omega=\Omega(r, \theta)$ is the planet's rotation, $\nu$ is a constant viscosity, and the Laplacian operator is given by

$$
\nabla^{2}=\frac{1}{r^{2} \cos ^{2} \theta} \frac{\partial^{2}}{\partial \phi^{2}}+\frac{1}{r^{2} \cos \theta} \frac{\partial}{\partial \theta}\left(\cos \theta \frac{\partial}{\partial \theta}\right)+\frac{1}{r^{2}} \frac{\partial}{\partial r}\left(r^{2} \frac{\partial}{\partial r}\right) .
$$

We have made an approximation neglecting some of the terms when going from a Laplacian of a vector to that of a scalar (Morse and Feshbach, 1953) in the viscosity term. Similar to the Boussinesq approximation, the large hydrostatic mean terms (2.3) can be removed from the vertical momentum equation so that the terms in the momentum equations tend to be of the same order. Typically in oceanic and atmospheric applications (Pedlosky, 1987), since the motion is confined to a thin spherical shell, some of the metric terms in (2.8 - 2.10) can be neglected. However, when studying the dynamics of a full sphere, where $r$ varies considerably, these terms are important. The Coriolis term associated with the vertical velocity and the Coriolis term in the vertical equation are typically neglected as well. The first is neglected due to the small aspect ratio between vertical lengths and horizontal lengths leading to the vertical velocity scaling smaller than the horizontal velocity. Similarly, due to the small aspect ratio the vertical momentum equation to the first order is hydrostatic (beyond the hydrostatic basic state) and the Coriolis term typically may be neglected. We emphasize that we do not make any of these approximations, and the importance 
of these typically neglected terms is discussed further in chapter 4 . In the deep sphere much of the intuition such as the vertical balance being close to hydrostatic, or the similar scaling of zonal and meridional motions does not hold. In fact, as we will show, in this problem there is a closer connection between the vertical and meridional velocities, than between horizontal ones.

\subsubsection{The Anelastic Equations for an Ideal Gas}

A main difference between the anelastic system and the Boussinesq one is that, since the background density is not taken as a constant, a more natural variable for the buoyancy is the entropy. We begin by discussing this for an ideal gas, following Ogura and Phillips (1962), and then show the buoyancy expression for a general equation of state. For an ideal gas we can express the entropy as a function of pressure and density $s=s(p, \rho)$ so that

$$
s=C_{p} \log \theta=C_{p} \log T-R \log p=C_{v} \log p-C_{p} \log \rho
$$

where $C_{p}$ and $C_{v}$ are the specific heat at constant pressure and volume for an ideal gas, and $R$ is the ideal gas constant. Considering a variation $s^{\prime}$ from the mean state $\bar{s}$ we can express the buoyancy term in (2.10) in terms of density and pressure using (2.13) so that

$$
\frac{\rho^{\prime}}{\bar{\rho}} \approx \frac{\rho^{\prime}}{\rho}=\frac{1}{\gamma} \frac{p^{\prime}}{p}-\frac{s^{\prime}}{C_{p}} \approx \frac{1}{\gamma} \frac{p^{\prime}}{\bar{p}}-\frac{s^{\prime}}{C_{p}},
$$

where $\gamma$ is the ratio $\frac{C_{p}}{C_{v}}$. Similarly we can do the same for the mean density gradient so that

$$
\frac{1}{\bar{\rho}} \frac{d \bar{\rho}}{d r} \approx \frac{1}{\gamma \bar{p}} \frac{d \bar{p}}{d r}-\frac{1}{C_{p}} \frac{d \bar{s}}{d r}=-\frac{g \bar{\rho}}{\gamma \bar{p}}-\frac{1}{C_{p}} \frac{d \bar{s}}{d r}
$$

where the approximation has been to the same level as the approximation done for the momentum equations in $(2.8-2.10)$. Then the vertical momentum equation $(2.10)$ 
using $(2.14,2.15)$ can be written as

$$
\begin{aligned}
\frac{D w}{D t}+2 \Omega \cos \theta u & =-\frac{\partial}{\partial r}\left(\frac{p^{\prime}}{\bar{\rho}}\right)+\frac{p^{\prime}}{\bar{\rho}}\left[\frac{g \bar{\rho}}{\gamma \bar{p}}+\frac{1}{C_{p}} \frac{d \bar{s}}{d r}\right]-g\left[\frac{1}{\gamma} \frac{p^{\prime}}{\bar{p}}-\frac{s^{\prime}}{C_{p}}\right] \\
& =-\frac{\partial}{\partial r}\left(\frac{p^{\prime}}{\bar{\rho}}\right)+\frac{p^{\prime}}{C_{p} \bar{\rho}} \frac{d \bar{s}}{d r}+\frac{g s^{\prime}}{C_{p}}
\end{aligned}
$$

so that the buoyancy term in the vertical momentum equation is expressed in terms of the entropy only. For a basic state which is adiabatic, this system is analogous to the Boussinesq system, with the pressure term including the variation in mean density, and entropy instead of density in the expression for buoyancy.

\subsubsection{The Anelastic Equations for a General Equation of State}

We would like to extend this to a general equation of state. Since our system diverges from an ideal gas in the interior (section 2.3), this will allow us to apply the anelastic equations to the deep interior of the planet. We assume a general equation of state, and define entropy in the general form $s=s(p, \rho)$. We use the following definitions

$$
\begin{gathered}
C_{p}=T\left(\frac{\partial s}{\partial T}\right)_{p}, \quad C_{v}=T\left(\frac{\partial s}{\partial T}\right)_{V}, \\
\alpha=-\frac{1}{\rho}\left(\frac{\partial \rho}{\partial T}\right)_{p}, \quad \delta=\frac{1}{\rho}\left(\frac{\partial \rho}{\partial p}\right)_{T},
\end{gathered}
$$

for the specific heats (at constant pressure and volume), the isobaric expansion co-

efficient and the isothermal compressibility per unit mass. This allows us to express the small entropy variation from a mean state as

$$
s^{\prime}=\left(\frac{\partial s}{\partial p}\right)_{\rho} p^{\prime}+\left(\frac{\partial s}{\partial \rho}\right)_{p} \rho^{\prime}=-\frac{C_{v} \delta}{T \alpha} p^{\prime}-\frac{C_{p}}{T \alpha \rho} \rho^{\prime} .
$$

Applying the same for the mean state entropy and keeping this derivation general, thus allowing the mean entropy to vary radially, gives

$$
\frac{d \bar{\rho}}{d r}=\frac{\alpha \bar{T} \bar{\rho}}{C_{p}} \frac{d \bar{s}}{d r}+\frac{C_{v} \delta \bar{\rho}^{2} g}{C_{p}}
$$


Then the vertical momentum equation can be written as

$$
\begin{aligned}
\frac{D w}{D t}+2 \Omega \cos \theta u & =-\frac{\partial}{\partial r}\left(\frac{p^{\prime}}{\bar{\rho}}\right)-\frac{p^{\prime}}{\bar{\rho}}\left[\frac{\alpha \bar{T} \bar{\rho}}{C_{p}} \frac{d \bar{s}}{d r}+\frac{C_{v} \delta \bar{\rho}^{2} g}{C_{p}}\right]-\frac{g}{\bar{\rho}}\left[-\frac{\alpha \bar{T} \bar{\rho}}{C_{p}} s^{\prime}-\frac{C_{v} \delta \bar{\rho}}{C_{p}} p^{\prime}\right] \\
& =-\frac{\partial}{\partial r}\left(\frac{p^{\prime}}{\bar{\rho}}\right)-\frac{\alpha \bar{T}}{\bar{\rho} C_{p}} \frac{d \bar{s}}{d r} p^{\prime}+\frac{g \alpha \bar{T}}{C_{p}} s^{\prime}
\end{aligned}
$$

Therefore, the buoyancy is expressed by two terms. One involves the mean state entropy gradient and the pressure variations, and a second term has only the entropy perturbations. This shows that a natural reference system, analogous to one of a constant background density in the Boussinesq system, would be an adiabatic reference state so that $\frac{d \bar{s}}{d r}=0$. In that case

$$
\frac{D w}{D t}+2 \Omega \cos \theta u=-\frac{\partial}{\partial r}(\Phi)+\frac{g \alpha T}{C_{p}} s^{\prime}
$$

where $\Phi=\frac{p^{\prime}}{\bar{\rho}}$ is the anelastic potential. In the case of an ideal gas (2.22) reduces to (2.17). We can gain more intuition for the buoyancy term by noting that

$$
\nabla \bar{T}=\left(\frac{\partial T}{\partial s}\right)_{p} \nabla \bar{s}+\left(\frac{\partial T}{\partial p}\right)_{s} \nabla \bar{p}=\frac{T}{C_{p}} \nabla \bar{s}-\frac{\alpha T g}{C_{p}}
$$

where we have used the basic hydrostatic state (2.3), and the Maxwell identity

$$
\left(\frac{\partial T}{\partial p}\right)_{s}=-\frac{1}{\rho^{2}}\left(\frac{\partial \rho}{\partial s}\right)_{p}
$$

Then for the adiabatic case we can write (2.22) as

$$
\frac{D w}{D t}+2 \Omega \cos \theta u=-\frac{\partial}{\partial r}(\Phi)-s^{\prime} \nabla \bar{T}
$$

Thus under the anelastic approximation, with an adiabatic background state, the buoyancy term is given directly by the entropy variation and the background temperature gradient. This result is the anelastic system used by Ingersoll and Pollard (1982) who have used a Legendre transform to obtain this relation directly, thus using the thermodynamic variables $s, T$ instead of $\rho, p$ which are typically used in geophysical fluid applications (which we will keep because of using the MITgcm). We have shown therefore that the anelastic approximation expressed in terms of entropy is 
not limited to an ideal gas, and if assuming an adiabatic reference state it takes the simple form (2.26).

\subsubsection{The Anelastic Thermodynamic Equation}

An advantage of this form is that it allows a direct connection to the thermodynamic equation, which in the most complete form for a general equation of state is written in terms of entropy so that

$$
\frac{D s}{D t}+\nabla \cdot(\kappa \nabla s)=\frac{Q}{T}
$$

where $Q$ is the heating rate per unit mass, and $\kappa$ is the diffusivity which we will assume to be constant. Then applying the anelastic approximation, and assuming a basic state which is adiabatic (constant $\bar{s}$ - see section 2.2.3), we can write the thermodynamic equation as

$$
\frac{\partial s^{\prime}}{\partial t}+\frac{1}{\bar{\rho}} \nabla \cdot\left(\bar{\rho} u s^{\prime}\right)-\kappa \nabla^{2} s^{\prime}=\frac{Q}{\bar{T}} .
$$

The forcing is described in section 2.5. For this system to be consistent for a general equation of state we need to show that the energy equation has a closed form.

\subsubsection{Energetics of the Anelastic System with a General Equa- tion of State}

In the Boussinesq system an energy equation can be derived by scalar multiplying the momentum equations with the velocity to form a kinetic energy equation. A potential energy equation can be formed by multiplying a buoyancy term with the thermodynamic equation. The evolution of the total energy can then be expressed as an energy flux. Ingersoll (2005) shows in an oceanic context with the density depending on three thermodynamic variables (pressure, temperature and salinity), the equations will still be energetically consistent. For the anelastic case Ogura and Phillips (1962) show that for an ideal gas a similar relation can be formed. We begin therefore from the momentum equation with the buoyancy in the vertical equation expressed in terms entropy $(2.22)$ 


$$
\frac{D \mathbf{u}}{D t}+2 \Omega \times \mathbf{u}=-\nabla\left(\frac{p^{\prime}}{\bar{\rho}}\right)-\frac{\alpha T p^{\prime}}{\bar{\rho} C_{p}} \nabla \bar{s}-s^{\prime} \nabla \bar{T},
$$

where $\mathbf{u}$ is the full $3 \mathrm{D}$ velocity. We define buoyancy and an anelastic potential as

$$
\begin{aligned}
b & =\frac{g \alpha T}{C_{p}} s^{\prime} \\
\Phi & =\frac{p^{\prime}}{\bar{\rho}} .
\end{aligned}
$$

Scalar multiplying (2.29) with $\bar{\rho} \mathbf{u}$ and using the anelastic mass equation (2.5) gives

$$
\frac{\partial}{\partial t}\left(\frac{\bar{\rho}}{2} \mathbf{u}^{2}\right)+\nabla \cdot\left[\bar{\rho} \mathbf{u}\left(\frac{\mathbf{u}^{2}}{2}+\Phi+\bar{T} s^{\prime}\right)\right]=\bar{\rho} \mathbf{u} \cdot \bar{T} \nabla s^{\prime}-\frac{\alpha T p^{\prime} w}{C_{p}} \frac{d \bar{s}}{d r}
$$

If the background state is adiabatic, so that the second term on the right hand side vanishes, we can use the thermodynamic equation (taking only conservative terms) to replace the right hand side of (2.32). Multiplying the thermodynamic equation (2.28) by $\bar{\rho} \bar{T}$ gives

$$
\bar{\rho} \bar{T} \frac{\partial s^{\prime}}{\partial t}=\bar{T} \nabla \cdot\left(\bar{\rho} \mathbf{u} s^{\prime}\right)=\bar{\rho} \mathbf{u} \cdot \bar{T} \nabla s^{\prime}
$$

where we have used the anelastic mass equation again. Then the energy equation can be written as

$$
\frac{\partial}{\partial t}\left[\bar{\rho}\left(\frac{\mathbf{u}^{2}}{2}+\bar{T} s^{\prime}\right)\right]+\nabla \cdot\left[\bar{\rho} \mathbf{u}\left(\frac{\mathbf{u}^{2}}{2}+\Phi-\bar{T} s^{\prime}\right)\right]=0
$$

Therefore for an adiabatic background state there is no requirement to use a specific equation of state for the anelastic equation to be energetically consistent.

\subsection{The Equation of State}

On Jupiter and Saturn the gas is primarily composed of hydrogen and helium with small amounts of heavier elements. At low temperatures and pressures in the outer regions of the planet, hydrogen is a molecular gas and the equation of state (EOS) may be approximated as an ideal gas. Deeper into the interior, however, due to the high 
densities and relatively low temperatures (compared to stars), the giant planets lie in an extremely complex thermodynamic regime. The main factors that separate the gas under these conditions from ideal gas behavior are pressure ionization, electron degeneracy, and Coulomb interactions (Guillot, 2005). We use an EOS calculated by Saumon et al. (1995) specifically for high pressure hydrogen and helium mixtures including these thermodynamic complexities. In addition this EOS has been partly calibrated with high pressure and density experimental data.

Below we review the physics governing this equation of state, estimating the effect of these phenomena on the pressure, given the density and temperature. Although this thesis focuses on the fluid dynamics we have devoted significant time to understanding the thermodynamics and estimating their importance on the equation of state and the reference state of the model. Eventually this boils down to a choice of an equation of state and the reference state discussed in section 2.4, but this choice was not obvious at start. In section 8.2.2 we estimate the gravitational moments of Jupiter using our model, which are a measurable quantity in the JUNO mission. These results may give further constraints on future equations of state.

\subsubsection{Electron Degeneracy}

For stars with mass over 0.3 solar, the typical densities and temperatures imply that the electrons will always behave with near Maxwellian distribution of the momentum. However, the Giant planets lie in a regime where due to the low mass, the temperatures are relatively cool, while the densities are high, and therefore the Pauli exclusion principle yields a distribution which is determined by Fermi-Dirac statistics. The number of electrons in a volume $d V$ and with the momentum $[p, p+d p]$ according to the Boltzmann distribution function is

$$
f(p) d p d V=\frac{4 n_{e} \pi p^{2}}{\left(2 \pi m_{e} k T\right)^{\frac{3}{2}}} e^{\left(\frac{-p^{2}}{2 m_{e} k T}\right)} d p d V
$$

where $k$ is the Boltzmann coefficient, $T$ is the temperature, $n_{e}$ is the number density of the electrons and $m_{e}$ is the electron mass. Then for a constant $n_{e}$ the maximum of the distribution function $p_{\max }=\sqrt{2 m_{e} k T}$ tends to smaller values of $p$ as temperature becomes smaller, and $f(p)$ becomes higher (since $n_{e}$ is given by $\int f(p) d p$ ). However, since electrons are fermions, for which Pauli's exclusion principle holds, each quantum 
cell of volume $d p_{x} d p_{y} d p_{z} d V=h^{3}$, where $h$ is the Plank constant, cannot contain more than two electrons. The Pauli's exclusion principle therefore demands that

$$
f(p) d p d V \leq \frac{8 \pi p^{2} d p d V}{h^{3}}
$$

and therefore giving an upper bound for $f(p)$. Figure 2.1 shows the Boltzmann distribution for different temperatures and the limit from the exclusion principle for both typical stellar values, and planetary interior values typical to Jupiter. It shows how due to the low temperatures the exclusion principle is a much stronger restriction for planetary values than for stellar ones, requiring the electrons to occupy much higher energy levels. Therefore the equation of state needs to include quantum mechanical
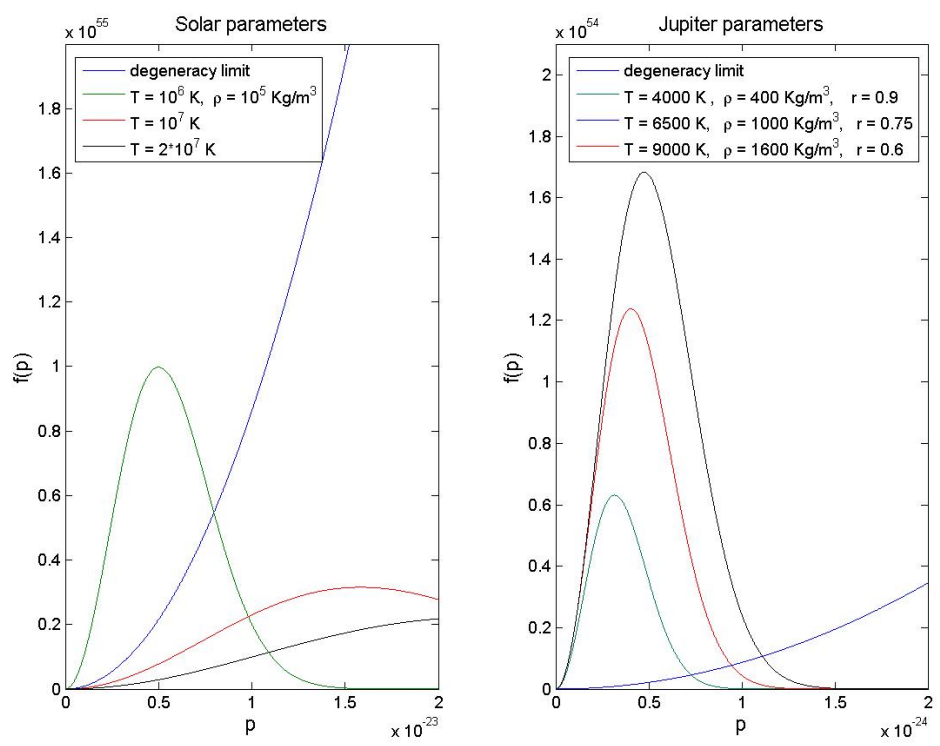

Figure 2.1: The Boltzmann distribution and Pauli's exclusion principle for both planetary and stellar values.

effects if the temperature is too low or the density is too high. Due to the relatively low temperatures in Giant planet interiors this happens relatively close to the exterior (Figure 2.3). These electrons are referred to as degenerate. The transition to a fully degenerate state is not a sharp one (for a finite temperature). The most probable occupation of the phase cells of the shell $[p, p+d p]$ in momentum space is determined by Fermi-Dirac statistics, where 


$$
f(p) d p d V=\frac{8 \pi p^{2} d p d V}{h^{3}} \frac{1}{1+e^{E / k T-\psi}}
$$

where $E=\frac{p^{2}}{2 m_{e}}$ is the energy in the non-relativistic case, and $\psi$ is defined as the degeneracy parameter. Then

$$
n_{e}=\frac{8 \pi}{h^{3}} \int_{0}^{\infty} \frac{p^{2} d p}{1+e^{E / k T-\psi}}=\frac{8 \pi\left(2 \pi m_{e} k T\right)^{\frac{3}{2}}}{h^{3}} a(\psi)
$$

with

$$
a(\psi)=\int_{0}^{\infty} \frac{\eta^{2} d \eta}{1+e^{\left(\eta^{2}-\psi\right)}}
$$

where we have defined $\eta=p\left(2 m_{e} k T\right)^{-\frac{1}{2}}$. Therefore the degeneracy parameter is a function of $n_{e} T^{-3 / 2}$ only. The limit of large negative values of $\psi$ represents the limit of high temperatures with a classic Boltzmann distribution. In the limit of large positive $\psi$, when introducing an energy so that $\psi=\frac{E_{0}}{k T}$, then for large enough $\psi$ there is a discontinuity in the distribution function at energy $E_{0}$. This corresponds to the limit of very low temperatures where there is a discontinuity at the Fermi energy. For intermediate values using $m_{e} d E=p d p$ and $p=\left(2 m_{e} E\right)^{\frac{1}{2}}$ the number density becomes

$$
n_{e}=\frac{4 \pi}{h^{3}}\left(2 m_{e} k T\right)^{\frac{3}{2}} F_{1 / 2}(\Psi)
$$

where

$$
F_{\nu}(\Psi)=\int_{0}^{\infty} \frac{\xi^{\nu} d \xi}{1+e^{(\xi-\psi)}}
$$

is the Fermi-Dirac function. The electron pressure is

$$
P_{e}=\frac{8 \pi}{3 h^{3}}\left(2 m_{e} k T\right)^{\frac{3}{2}} k T F_{3 / 2}(\Psi)
$$

Therefore for a given density and temperature, by inverting (2.38) (the Fermi-Dirac integrals have a unique inverse function), the electron pressure $P_{e}$ can be determined. In Figure 2.2 we show the pressure of the electrons due to degeneracy as function of 
temperature and density. Superimposed is the reference state for Jupiter. It shows that Jupiter lies in the region where degeneracy is important, where the density has a stronger effect than temperature on pressure. Figure 2.2 shows that for Jupiter the effect of electron pressure is important and, over most of the domain is more important than the pressure of the atoms themselves approximated by the ideal gas pressure.
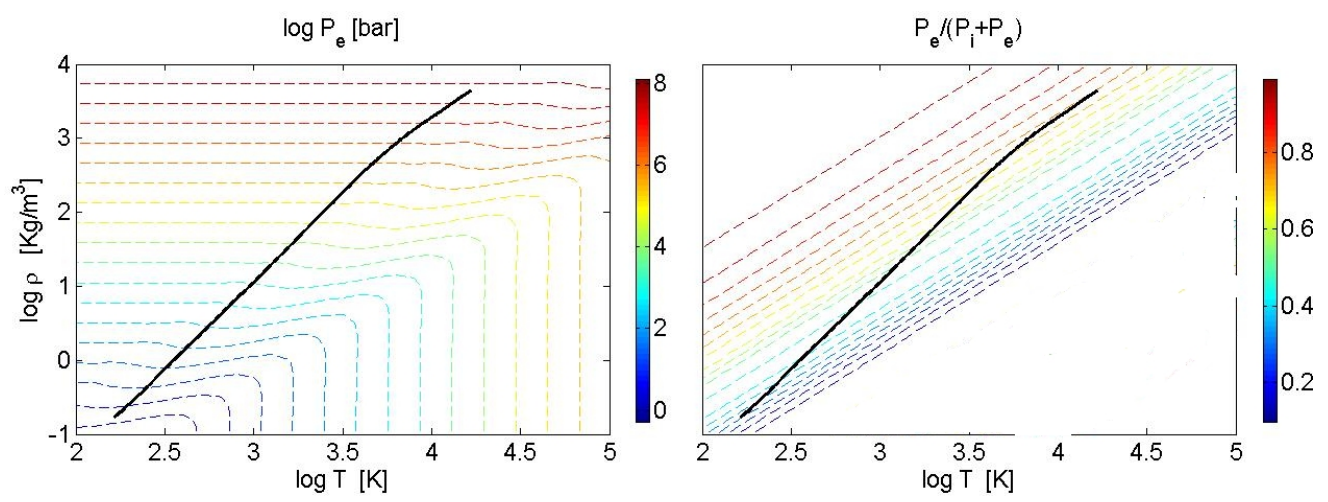

Figure 2.2: The effect of electron pressure on the equation of state. left: The pressure $P_{e}$ of the electrons as function of temperature and density. The black line is the profile for Jupiter from Guillot and Morel (1995). right: The relative contribution of electron pressure to the total pressure of an ideal gas of Hydrogen.

\subsubsection{Pressure Ionization}

The ionization level of an atom is determined by its temperature and pressure. This is usually given by the Saha relation (Kippenhahn and Weigert, 1990) which holds for high temperatures in the interiors of stars. However, in Jupiter's interior most of the ionization is due solely to pressure. This is called pressure ionization and can be approximated roughly by the fact that an atom must be ionized if the matter is so dense that the distance between atoms is smaller than twice the Bohr radius. In this case even an electron in the lowest possible orbit will not be bound. The condition for pressure ionization could be approximated as

$$
d=\left(\frac{3}{4 \pi n_{H}}\right)^{\frac{1}{3}}<2 a_{0},
$$


where $d$ is the distance between atoms, $n_{H}$ is the number density, and $a_{0}$ is the Bohr number. For hydrogen this leads to an ionization density of $348 \frac{\mathrm{Kg}}{\mathrm{m}^{3}}$ which corresponds approximately to 0.92 of the radius of Jupiter and 0.8 for Saturn (Figure 2.3). Therefore we can expect the deep interior to be completely ionized. Even in the regions exterior to the radius of full ionization, the ionization level will still be heavily influenced by pressure ionization. In the exterior, where pressure ionization is negligible, calculations from the Saha relation show that temperatures are too low to cause significant thermal ionization. To estimate the pressure therefore one needs to take into account the pressure both from the ions and the electrons. An order of magnitude estimate is that ions and electrons have similar contributions to the total pressure (Guillot, 2005).

Thus most of the interior is composed of heavily ionized dense plasma, often referred to as liquid metallic hydrogen. The physics of the phase transition between molecular fluid to the metallic fluid caused by the pressure ionization remain poorly understood. There have been attempts to calculate an equation of state for this phase transitional regime (Saumon et al., 1995) however recent results by the authors themselves suggests that their previous results were not accurate. Therefore in the equation of state we will use we include the effect of pressure ionization, but ignore any variations in the equation of state from processes involved in the phase transition itself.

\subsubsection{Coulomb Interactions}

Another important quantity that has an effect on the equation of state is the coupling parameter, which is the ratio of the Coulomb potential to the thermal energy. This measures how strong are the coulomb interactions relative to the thermal energy as the density changes in the planet's interior. The coupling parameter for hydrogen is given by

$$
\Gamma=\frac{e^{2}}{d k T}=\frac{e^{2}}{k}\left(\frac{4 \pi}{3 n_{H}}\right)^{\frac{1}{3}} \frac{\rho^{\frac{1}{3}}}{T},
$$

where $d$ is the mean distance between nuclei, and $e$ is the electron charge (Guillot, 2005). As $\Gamma$ increases due to either an increase in density or a decrease in temperature Coulomb forces become stronger. Hubbard (1968) has shown that Jupiter's interior is 
not expected to crystallize (happens for $\Gamma>180$ ), and should be hot enough so that the interior will remain a fluid. Saturn's interior is also expected to remain a fluid. Typical values for the interior can be seen in Figure 2.3, and the system is dominated by the repulsive Colombian potential between nuclei.

\subsubsection{The SCVH Equation of State}

In summary a large fraction of the interior is composed of metallic hydrogen. In this region electron degeneracy, pressure ionization, and Coulomb interactions have significant contributions to the pressure. Outside of this region hydrogen is a molecular gas, and to a good approximation is close to an ideal gas. In the interior the pressure can be expressed in the following form (Stevenson, 1991)

$$
P=P_{e}+P_{i o n}+P_{c o u l}+P_{e x}
$$

where $P_{e}$ is the contribution from the degenerate electron gas, $P_{i o n}$ is the contribution from the ions, $P_{\text {coul }}$ is a negative term due to the Coulombian interactions of nuclei, and $P_{e x}$ is a negative term due to electron-electron repulsion because of the exclusion principle. Exact calculations of these effects are complex and involve further approximations that until recently have been untested in the appropriate regimes of temperature and pressure. Several recent experiments on hydrogen (Collins et al., 1998; Knudson et al., 2001) now provide data in regimes of interests for giant planets and can provide constraints on the equation of state. Saumon et al. (1995) have calculated an approximate equation of state (referred to as SCVH), for both hydrogen and helium taking into account all these effects and extrapolating between the different regimes.

In Figure 2.3 we compare between the SCVH equation of state for hydrogen (blue), and an ideal gas (dashed red). To get a feel for rough estimates of the physics diverging the equation of state from an ideal gas, we show the limits for the phenomenon discussed in this section. The green lines show the thermal and pressure ionization limits (2.43), the purple curve shows the electron degeneracy limit (2.42), and the magenta curves show the Coulomb limits (2.44) for different values of $\Gamma$. It is clear that beyond $10^{4}$ bars ( $2 \%$ of the planetary radius) all these effects become important and indeed beyond this region the SCVH EOS diverges from an ideal gas. In the low temperature and density limit the SCVH EOS is similar to an ideal gas, while for 


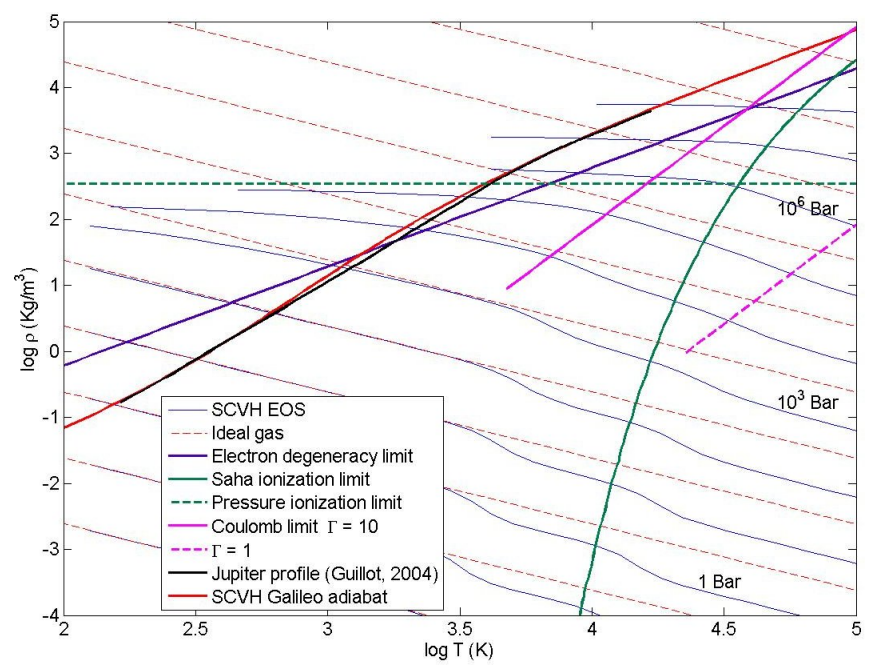

Figure 2.3: Isobars of the hydrogen SCVH EOS and an ideal gas in $\log \rho-\log T$ space. black: the profile for Jupiter from Guillot and Morel (1995); red: the adiabat of the SCVH EOS that matches the Galileo observation; purple: the limit where pressure from the electron gas becomes significant (2.42); dashed green: the limit of pressure ionization (2.43); green: the limit of ionization from the Saha relation; pink: where Coulomb interactions are significant with $\Gamma=10$ (2.44); dashed magenta: the Coulomb limit with $\Gamma=1$.

high pressures it differs significantly.

In comparison with hydrogen, the EOS of helium under the conditions of interest for the giant planets has been less studied. Experimental data for helium is only available up to $0.56 \mathrm{Mbar}$ (Nellis et al., 1984). A major complication (Salpeter, 1973) is that hydrogen and helium mixtures can undergo a phase separation where the heavier helium will form droplets that will fall towards central regions of the planets. Nonetheless, Saumon et al. (1995) have computed an EOS for helium, though it has not been compared against experimental data. This should not affect the results too much since for giant planet composition mixtures, hydrogen represents about $90 \%$ of the atoms, and helium about $10 \%$. The consequent EOS for hydrogen-helium mixtures is then calculated using the additive volume rule such that

$$
\rho^{-1}=(1-Y) \rho_{H}^{-1}+Y \rho_{H e}^{-1}
$$

where $Y$ is the helium mass fraction. Then the coefficients in (2.20) can be calculated based on this rule. This method implicitly neglects any interactions between hydrogen and helium. 
Given that by using the SCVH equation of state for hydrogen, we are already making a big step beyond the Boussinesq and ideal gas models, we will not add at this stage the complexities and uncertainties of the hydrogen-helium mixtures. Using the hydrogen SCVH equation of state should be sufficient for the level of complexity of our model. For example, the ideal gas constant for giant planet composition mixtures (which is relevant for the outer regions - Figure 2.3), will change by less than $10 \%$ when comparing it to the ideal gas constant of only hydrogen. The uncertainty in the other parameters of our model will be probably larger than the discrepancy between the equation of states with and without the helium component (see section 6.3). In addition, we will not account for the variations in the hydrogen EOS at the hydrogen phase transition that occurs between the molecular and metallic fluid. The equation of state for this phase transition has been published with the original SCVH paper, but the authors have recently reported an error in that calculation.

\subsection{The Reference State}

As discussed in section 2.2.3, using an adiabatic reference state implies that the anelastic system is energetically consistent. We have shown that this does not limit the form of the equation of state and, for a convective driven interior, is therefore a reasonable approximation. The Galileo entry probe has found the atmosphere to be close to a dry adiabat beyond the 1 bar level (Seiff et al., 1997). We find that, when taking this value of entropy from the Galileo probe measurement, and using it as the adiabat with the SCVH EOS, the adiabatic profile matches well previous estimates of the interior mean density-temperature-pressure profile (Guillot and Morel, 1995). We therefore use this "Galileo adiabat" as our reference state for the model. The details of understanding of the interior depend on variations in the adiabacity of the fluid as suggested by Guillot et al. (1994). However, for the level of sophistication of this fluid dynamical model, we feel this constant entropy basic state will suffice. The variation from this reference entropy is computed dynamically.

The vertical grid is chosen so that grid spacing follows a constant mean pressure ratio between levels. Relating each pressure level to its vertical depth is set following calculations of Guillot and Morel (1995), and Guillot et al. (2004). Once the constant entropy $(\bar{s})$, and the mean reference pressure for every vertical grid point are set, the reference temperature and density can be found from the SCVH EOS. Integrating the 
reference density allows calculating the gravitational acceleration for the vertical grid (2.4). Figure 2.4 shows these reference fields as a function of depth. In fact for the dynamics only $\bar{\rho}(r)$ and $g(r)$ come in, where the $\bar{T}(r)$ is used only in the calculation of the forcing profile (section 2.5). For every layer separately we then fit a polynomial to the SCVH EOS for the variation in density so that
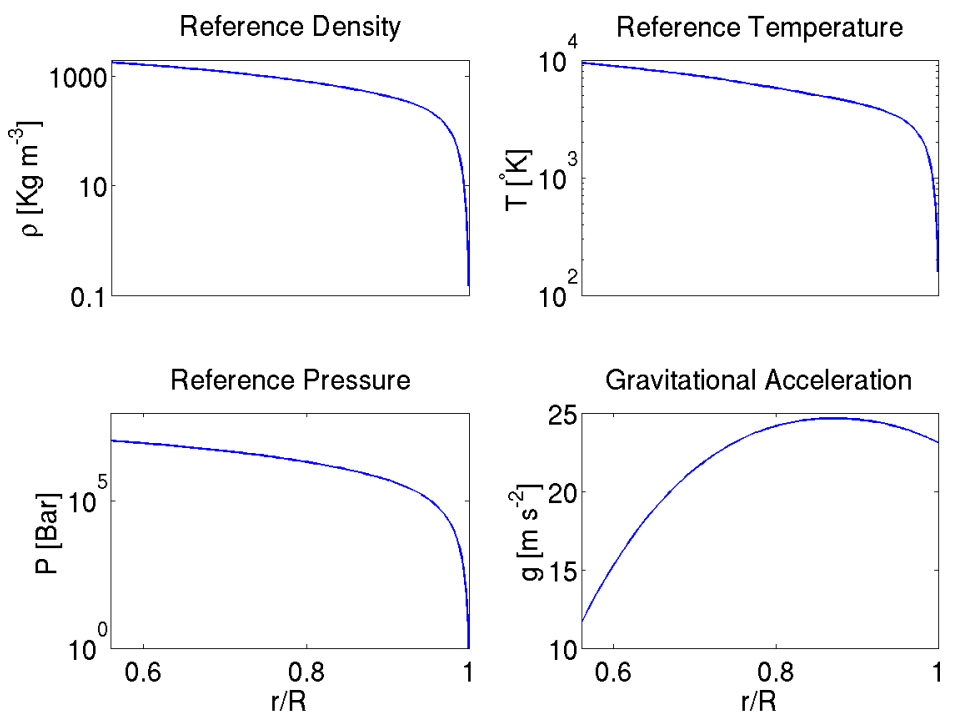

Figure 2.4: The adiabatic reference state of the model. Plots of density, temperature, pressure (logarithmic axis), and gravitational acceleration as a function of depth.

$$
\rho(s, p)=\bar{\rho}+\left(\frac{\partial \rho}{\partial s}\right) s^{\prime}+\left(\frac{\partial \rho}{\partial p}\right) p^{\prime}
$$

where the derivatives are calculated from the SCVH polynomial for each reference pressure (see Appendix A), and $s^{\prime}$ and $p^{\prime}$ come dynamically from the model. This variation in density feeds back to the model dynamics. Thus we have a fully coupled fluid dynamic-thermodynamic system. To the best of our knowledge this is the first time such an elaborate EOS has been incorporated to a dynamical gas-giant model. We feel the modification of the density-pressure-temperature-entropy relationship will be a considerable improvement to the existing dynamical models, and will give a much better representation of the planet's interior and its interactions with the outer atmosphere. As discussed in chapter 1 since the gas is largely ionized in the deep interior the magneto-hydrodynamic contributions which we do not include may be significant as well. 
The pressure-temperature-density relationship is shown in Figure 2.5, where it can be seen that up to about 1 Mbar (0.9 the radius of the planet) the SCVH EOS is close to an ideal gas, but it differs substantially for the deep interior.

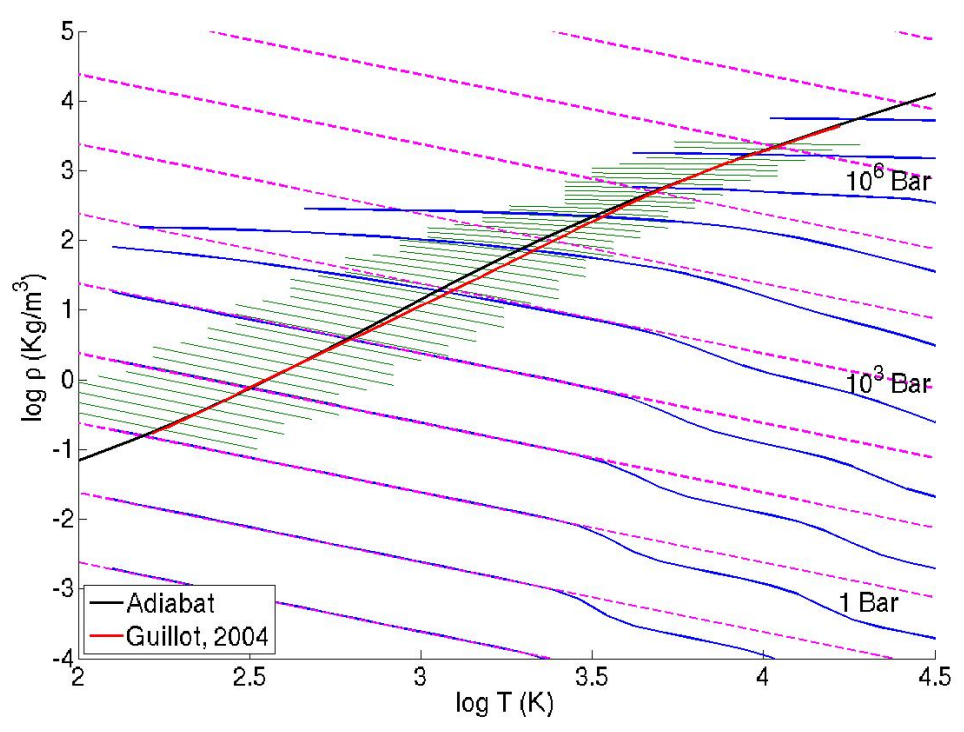

Figure 2.5: Contours of pressure in $\log \rho \log T$ space for the SCVH EOS (blue) and an ideal gas (magenta). The adiabatic reference state (black) is close to the calculations (red) of Guillot and Morel (1995); Guillot et al. (2004). The model uses a different polynomial for each layer (green) to calculate the dynamical density (2.47).

\subsection{Forcing}

The fact that Jupiter emits more energy than it receives from the sun implies that internal heat is transported from the planet's interior to space. The structure of the dynamics is related to the mechanisms transporting the heat. In stars heat is often transported by radiation and conduction. On Jupiter it is estimated that convection rather than conduction is in effect what is transporting heat (Guillot et al., 2004).

The forcing as applied to the model assumes the vertical profile is close to adiabatic and that the planet is cooling on long time scales. Suppose we allow for $\bar{s}$ to vary on long time scales so that its variation represents the long time cooling of the planet. We assume that transport of heat is diffusive so the heating has the form

$$
Q=C_{v} \kappa \nabla^{2} \bar{T} .
$$


Over the long non adiabatic time scales the forcing is given by

$$
\frac{D \bar{s}}{D t}=\frac{\partial \bar{s}}{\partial t}=\frac{C_{v} \kappa \nabla^{2} \bar{T}}{\bar{T}}
$$

The vertical profile of the heating rate (2.49) is shown in Figure 2.6. We constrain the heating so that, when integrated over the whole volume, the total forcing will be zero, and thus no net heat is added (or lost) from the system at every time step. Therefore we shift the conduction profile (2.49) so that the net heating is zero, and the difference is the heating associated with the long time scale cooling. Then the actual long time scale cooling is given by

$$
\frac{\partial \bar{s}}{\partial t}=C_{v} \kappa\left\langle\frac{\nabla^{2} \bar{T}}{\bar{T}}\right\rangle
$$

where we denoted the difference between the original profile (2.49) and the shifted vertical heating profile by \langle\rangle . This is the representation of the long time cooling of the planet, and this term represents the net loss of energy which is seen in observations. Then the thermodynamic equation (2.28) including the explicit forcing becomes

$$
\frac{\partial s^{\prime}}{\partial t}+\frac{1}{\bar{\rho}} \nabla \cdot\left(\bar{\rho} \mathbf{u} s^{\prime}\right)-\kappa \nabla^{2} s^{\prime}=C_{v} \kappa\left(\frac{\nabla^{2} \bar{T}}{\bar{T}}-\left\langle\frac{\nabla^{2} \bar{T}}{\bar{T}}\right\rangle\right)
$$

The heat flux $(F)$ is related to the heating rate by $\frac{Q}{T}=\nabla \cdot F$. Hence, we can calculate the effective flux at each depth from the heating by

$$
F=\frac{1}{r^{2}} \int \frac{\bar{\rho} Q}{\bar{T}} r d r+F_{0}
$$

where $F_{0}$ is zero since the flux at the bottom is zero. The normalized heating rate and heat flux are shown in Figure 2.6. Note that the flux out of the atmosphere is effectively zero, which is different from Rayleigh-Benard type convection models (e.g. Heimpel et al., 2005) that have very high outgoing heat fluxes. The interior heat fluxes are very large but compensate for the use of eddy viscosity terms which are big due to the size of the grid. We discuss this issue more in section 4.7. 


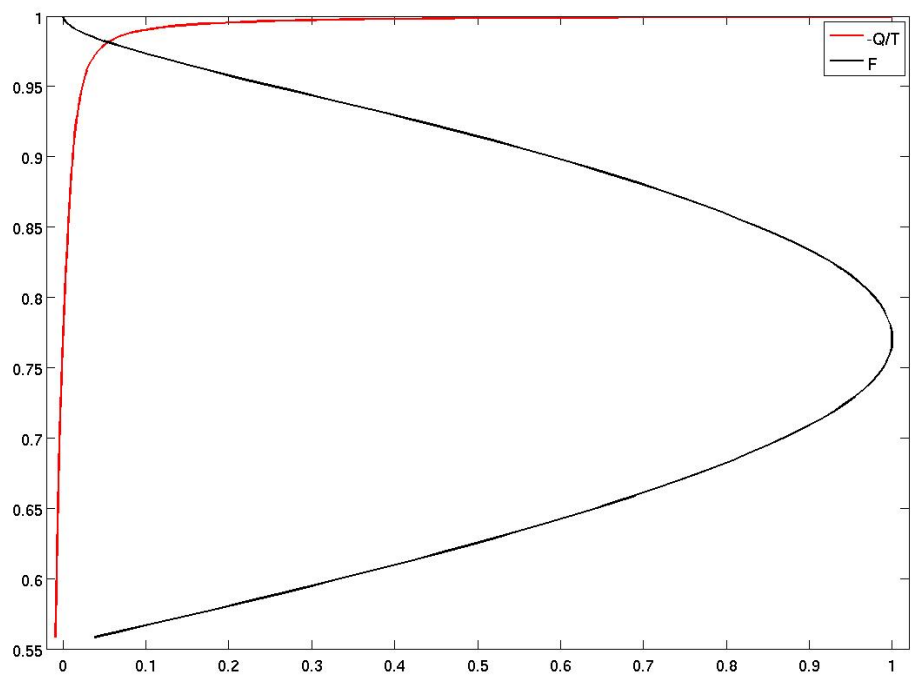

Figure 2.6: The applied heating function (red) and the resulting heat flux (black) as a function of depth. Both are normalized (note that the heating is negative so that the top levels are effectively cooling and the bottom are heating). The integrated forcing (4.29) over the whole domain is zero.

\subsection{Model Summary}

The model solves the full spherical momentum equations with no spherical shell approximations. The mass equation contains compressibility of the mean density which varies radially. The thermodynamic equation is used in terms of entropy and contains both advection and diffusion of entropy. The equation of state for the variation in density includes both entropy and pressure fluctuations, and the vertically dependent coefficients are given by the SCVH equation of state. This forms a system of six equations $\left(2.8,2.9,2.10,2.7,2.28\right.$, and 2.47) solved for the six unknowns $u, v, w, s^{\prime}, \rho^{\prime}$, and $p^{\prime}$. The gravitational acceleration $g(r)$ is calculated from the mean density. These equations have the parameters $\Omega, \nu, \kappa$ and $Q$. These parameters are set by three nondimensional numbers which control the system; the Prandtl (viscosity vs. conductivity), Taylor (rotation vs. viscous damping) and Rayleigh numbers (buoyancy vs. viscous and thermal damping). These numbers are given by

$$
\operatorname{Pr}=\frac{\nu}{\kappa}, \quad T a=\frac{4 \Omega^{2} H^{4}}{\nu^{2}}, \quad R a=\frac{B_{0} H^{4}}{\nu \kappa},
$$


where $H$ is the total vertical extent of the model, and $B_{0}$ is given by

$$
B_{0}=\frac{Q}{\bar{s} \bar{T}_{0}}\left(\frac{g_{0}}{H}\right)^{\frac{1}{2}}
$$

where the subscript 0 denotes the top level. The heating therefore is normalized by the reference entropy value $\bar{s}$, and thus reduces the system dependence on the specific choice of the value of $\bar{s}$ (although this choice still sets the other reference values). $B_{0}$ will therefore be the equivalent of the Brunt-Vaisala frequency in a stratified fluid. To keep the parameter range simple and since the grid spacing is fairly uniform (aka horizontal scales are similar to vertical scales), we use the same viscosity and diffusivity parameters in all the equations. Often in the text we will use the Ekman number $E k=\frac{\nu}{\Omega H^{2}}$ instead of the Taylor number. Other model settings, which we experiment with are the total vertical depth (ranging from a thin spherical shell to $93 \%$ of the planet radius - section 6.1 ), and the rotation rate. Since we consider several forms of thermal forcing, we may have more than one Rayleigh-like number, e.g., one measuring the horizontal variation in heating in the top layers. 


\section{Chapter 3}

\section{Numerical Results}

\subsection{Axisymmetric Results}

When we observe the circulation on the giant planets it appears to first order fairly zonally symmetric. From a modeling point of view the question is can an axisymmetric model capture the main features of this circulation such as the equatorial superrotation, alternating jets and poleward heat transport? From our experience with Earth's atmosphere we know that zonally symmetric models had success in explaining some of the features of the general circulation (e.g. Held and Hou, 1980), but eddy fluxes are crucial in understanding the general circulation (Schneider, 2006).

In this section we present results of axisymmetric calculations. We use the full 3D model but truncate it to one grid point in the zonal direction. Much of the model development was done in the axisymmetric setup, which is simpler computationally and still contains the vertical modifications that were made to the MITgcm. When comparing to the 3D results we find that the circulation is quite different. Nevertheless comparing the $2 \mathrm{D}$ to the $3 \mathrm{D}$ results illuminates the role of the zonal asymmetries, particularly the role of the eddies in driving the equatorial superrotation. Some aspects of the circulation do carry over from the 2D to the 3D model and we focus on those in the first subsections. We will begin by discussing the effect of rotation on the circulation and then discuss the onset of convection and the critical Rayleigh number. 


\subsubsection{The Effect of Rotation on Convective Plumes}

In a non rotating system the intuition about convecting plumes is simple, and convection is associated with fluid motion along the direction of the gravity vector. In a rotating system the Taylor-Proudman theorem puts constraints on the dynamics, where now two key players in determining the direction of motion of a convecting plume, will be the direction of the rotation and gravity vectors.

In many geophysical models due to the traditional small aspect ratio approximation the horizontal component of the rotation vector is neglected. However even for deep oceanic convection the aspect ratio within the convection columns may be near unity (Lilly et al., 1999; Marshall and Schott, 1999). Then the vertical velocities are comparable to horizontal ones so that this approximation is not valid. In the case of a deep convective atmosphere this is not valid as well. The traditional approximation treats the rotation and gravitation vectors as parallel; the issue of convection when they are not has been addressed in several studies. Numerical experiments by several authors (e.g. Zhang and Schubert, 1997) have shown alignment of convective flow with the rotation axis. This issue is not simple to treat in laboratory experiments because the difficulty of creating a finite angle between the rotation and gravity vectors, and the need of having the center of gravity not coinciding with the center of the Earth. However as suggested by Busse et al. (1998) the angle between the buoyancy force and the rotation axis can be produced by the use of centrifugal force. Sheremet (2004) used this method and found out that oceanic type sinking plumes tend to sink in an intermediate direction between the effective gravity and the rotation and shift eastward. In a space lab experiment Hart (1985) used a spherically symmetric electric field acting on a dialectrically insulating liquid to simulate gravity in space, and address the issue of the direction of the plumes in a rotating system.

In this section we show results from the axisymmetric model showing the effect of rotation on the convectively driven flow. Simplifying the model further, in this section we use Boussinesq dynamics. In section 4.5 we discuss the effect of rotation on the anelastic model and show the $3 \mathrm{D}$ case, but the essence is captured by the axisymmetric Boussinesq model. This analysis in 2D is simpler also because we can define a 2D streamfunction, which will describe the motion in the radial-meridional plane. In the 3D case we can do this only in cases where rotation limits the motion

to be $2 \mathrm{D}$. Without assuming a small Rossby number we can write the steady state 

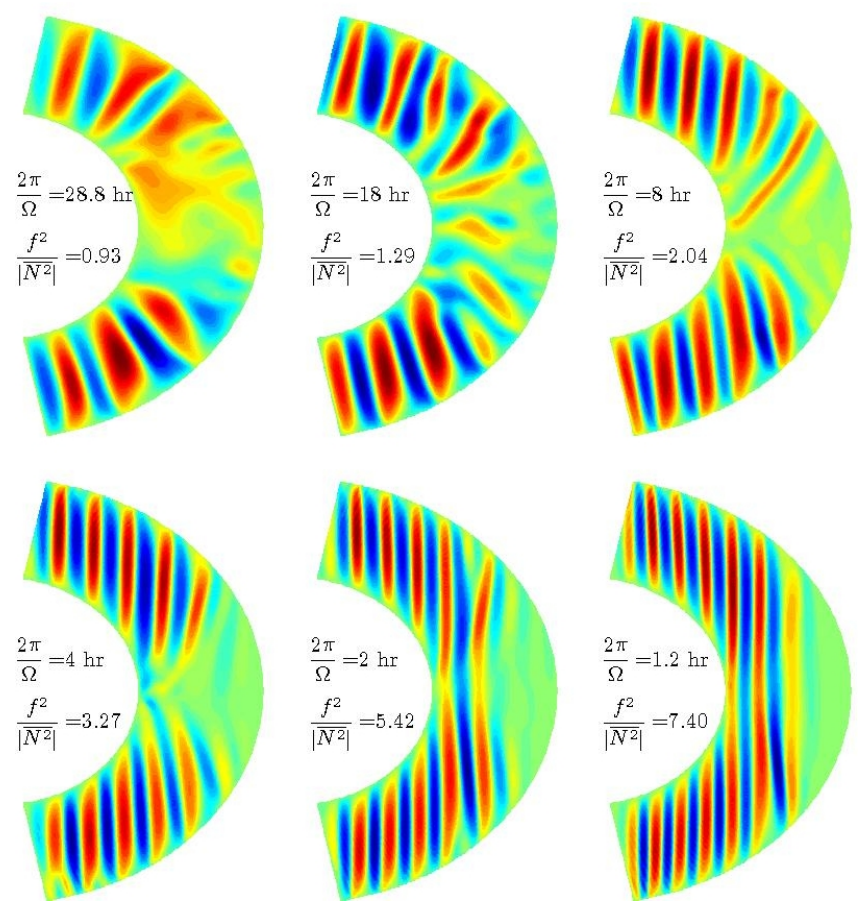

Figure 3.1: The meridional streamfunction for axisymmetric experiments with different rotation periods.

vorticity equation (Pedlosky, 1987) as

$$
2 \Omega \cdot \nabla \mathbf{u}+[\omega \cdot \nabla \mathbf{u}+\nabla \cdot(\omega \mathbf{u})]=\frac{1}{\rho_{0}} \nabla \rho^{\prime} \times g
$$

where $\omega=\nabla \times \mathbf{u}$ is the vorticity vector, $\mathbf{u}$ is the $3 \mathrm{D}$ velocity vector and $\rho_{0}$ is the constant density. If the flow were completely barotropic then for small Rossby numbers (or rapid enough rotation), (3.1) would be dominated by the first term. The Taylor-Proudman theorem then implies that the velocity is constant along the direction of the rotation axis. However, since the convection drives plumes with horizontal gradients the flow is not completely barotropic. For slow rotation the vorticity flux and tilting will balance the baroclinic vorticity production term. For cases of weak enough convection we find that though locally the Brunt-Vaisala frequency can vanish (in the plumes), on average over the domain it has a positive (small) value. Therefore the two physical time scales in the problem, the rotation period, and the buoyancy period, set the character of the flow. For large $\frac{\Omega^{2}}{N^{2}}$ the flow will be dominated by the rotation, and the plumes will align with the axis of rotation giving nearly constant 


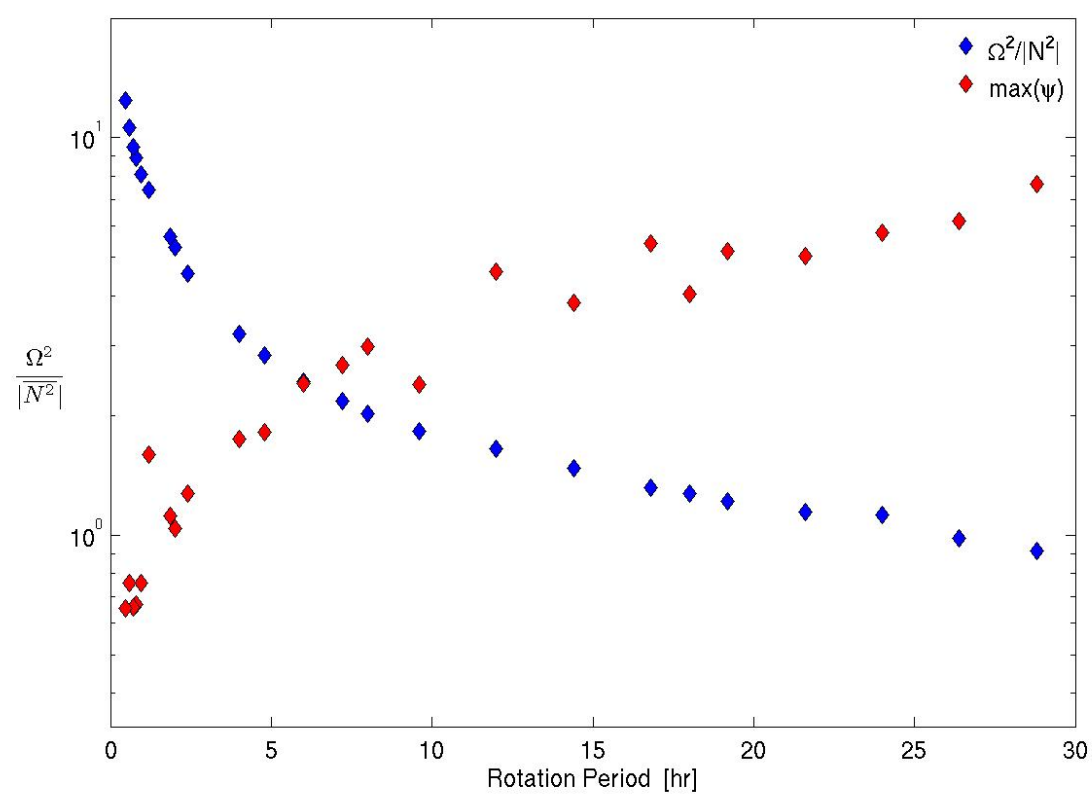

Figure 3.2: The ratio $\frac{\Omega^{2}}{\left|N^{2}\right|}$ (blue), and the maximum value of the streamfunction (red) as function of the rotation period for axisymmetric runs.

velocities along this axis. When $\frac{\Omega^{2}}{N^{2}}$ is small the buoyancy dominates the rotation and the plumes align in the direction of the gravity vector. Figure 3.1 shows the 2D radial-latitudinal streamfunction for axisymmetric cases with different rotation period. The flow develops circulation cells that change their character based on the ratio of $\frac{\Omega^{2}}{N^{2}}$. Figure 3.2 shows this ratio as a function of the rotation period for a series of runs varying only in rotation period. For strongly convective flow the buoyancy frequency will not be a good measure of convection. An equivalent measure of the convection can be the ratio of the nondimensional numbers

$$
\chi \equiv \frac{T a \cdot \operatorname{Pr}}{R a}=\frac{4 \Omega^{2}}{B_{0}}
$$

where $B_{0}$ has been defined in (2.54). We show in section 4.5 for the $3 \mathrm{D}$ case that this is a good measure to characterize the flow: thus when $\chi>1$ the flow is rotation dominated and aligns with axis of rotation, and when $\chi<1$ it is not. We discuss this further in section 4.5. Figure 3.2 also shows the normalized intensity of the $2 \mathrm{D}$ streamfunction.

The zonal velocity character is very different from the zonal velocity in the $3 \mathrm{D}$ 
case. This velocity structure is shown in Figure 3.4 where we compare the $2 \mathrm{D}$ to the 3D flow. The results shown in this section are for an Earth size aqua-planet (a developmental stage of this model) so numerical values can not be compared between this section and the rest of the thesis.

\subsubsection{The Critical Rayleigh Number for a Rotating Fluid on a Sphere}

We study the onset of convection in the rotating axisymmetric system. This again is a case where the axisymmetric results do not differ much from the spherical ones, and to simplify the analysis we look at the Boussinesq case. We look at the onset through a local linear stability analysis and compare the result to numerical axisymmetric results. The linear system in spherical geometry is given by

$$
\begin{aligned}
\frac{\partial u}{\partial t}-2 \Omega \sin \theta v+2 \Omega \cos \theta w & =\nu \nabla^{2} u \\
\frac{\partial v}{\partial t}+2 \Omega \sin \theta v & =-\frac{1}{r \rho_{0}} \frac{\partial p}{\partial \theta}+\nu \nabla^{2} v \\
\frac{\partial w}{\partial t}-2 \Omega \cos \theta u & =-\frac{1}{\rho_{0}} \frac{\partial p}{\partial r}+b+\nu \nabla^{2} w \\
\frac{1}{r} \frac{\partial v}{\partial \theta}+\frac{\partial w}{\partial r} & =0 \\
\frac{\partial b}{\partial t}+w S & =\kappa \nabla^{2} b
\end{aligned}
$$

where $b=-\frac{g \rho^{\prime}}{\rho_{0}}$ is the buoyancy and the rest of the variables and parameters are defined in 2.2 . We assume that locally we can describe the perturbation by the form

$$
[u, v, w, b, p]=\left[u_{0}, v_{0}, w_{0}, b_{0}, p_{0}\right] e^{i(l \theta+m r-\sigma t)}
$$

which allows writing this system as

$$
\left(\begin{array}{ccccc}
-i \sigma-\frac{v a^{2}}{r^{2}} & -2 \Omega \sin \theta & 2 \Omega \cos \theta & 0 & 0 \\
2 \Omega \sin \theta & -i \sigma-\frac{v a^{2}}{r^{2}} & 0 & 0 & -\frac{i l}{\rho_{0} r} \\
-2 \Omega \cos \theta & 0 & -i \sigma-\frac{v a^{2}}{r^{2}} & -1 & -\frac{i m}{\rho_{0}} \\
0 & -\frac{i l}{r} & -i m & 0 & 0 \\
0 & 0 & S & -i \sigma-\kappa \frac{v a^{2}}{r^{2}} & 0
\end{array}\right)\left(\begin{array}{c}
u_{0} \\
v_{0} \\
w_{0} \\
b_{0} \\
p_{0}
\end{array}\right)=0,(3.9)
$$


where we have approximated the Laplacian operator by dropping first order derivatives and denoted the total wavenumber $a^{2}=-\left(l^{2}+r^{2} m^{2}\right)$. Solving this system for $\sigma=0$ gives the critical value for instability. This critical value occurs at

$$
S=\frac{\kappa}{\nu} \frac{(2 \Omega \cos \theta l+2 \Omega \sin \theta r m)^{2}}{l^{2}}+\frac{a^{6} \kappa \nu}{r^{4} l^{2}}
$$

and gives an expression for the critical Rayleigh number

$$
R a_{C}=\left(\frac{2 \Omega}{\nu}\right)^{2} \frac{r^{4}(l \cos \theta+r m \sin \theta)^{2}}{l^{2}}+\frac{a^{6}}{l^{2}} .
$$

Therefore the critical Rayleigh number is composed of two terms. The first depends on the rotation period, and the other purely on the wave numbers. In the limit of slow rotation the solution is dominated by the second term implying that the onset of convection does not depend on latitude. The solution in this limit is the classical critical number for Rayleigh-Benard convection (e.g. Chandrasekhar, 1961) for the case where the zonal wave number is zero. Busse (2002) studies the onset of convection in an annulus and finds a similar structure to the critical Rayleigh number, though with no latitudinal dependence due to the different geometry. In the limit of rapid rotation if the first term dominates then the onset of convection will depend on latitude.

We can test this solution using the numerical model. To allow quantification of the dependence of the onset of convection on latitude we use a simplified forcing. Instead of forcing by the profile shown in Figure 2.6 we apply a heat flux to the bottom boundary, which is relaxed by Newtonian cooling at the top. We assume the latitudinal number of plumes is related to the meridional wave number, and then can plot the intensity of the plumes during the initial stages of convection as a function of latitude. In Figure 3.3 we compare the outbreak of the convective plumes as a function of latitude and compare that to the inverse of the critical function obtained in (3.11). The bottom panel shows a qualitative match between the two profiles. The intensity of the convection is stronger towards the poles where the critical Rayleigh number is smaller. When we look at the spin-up of the model with more complicated schemes of forcing we see also stronger initial convection at higher latitudes. We find 


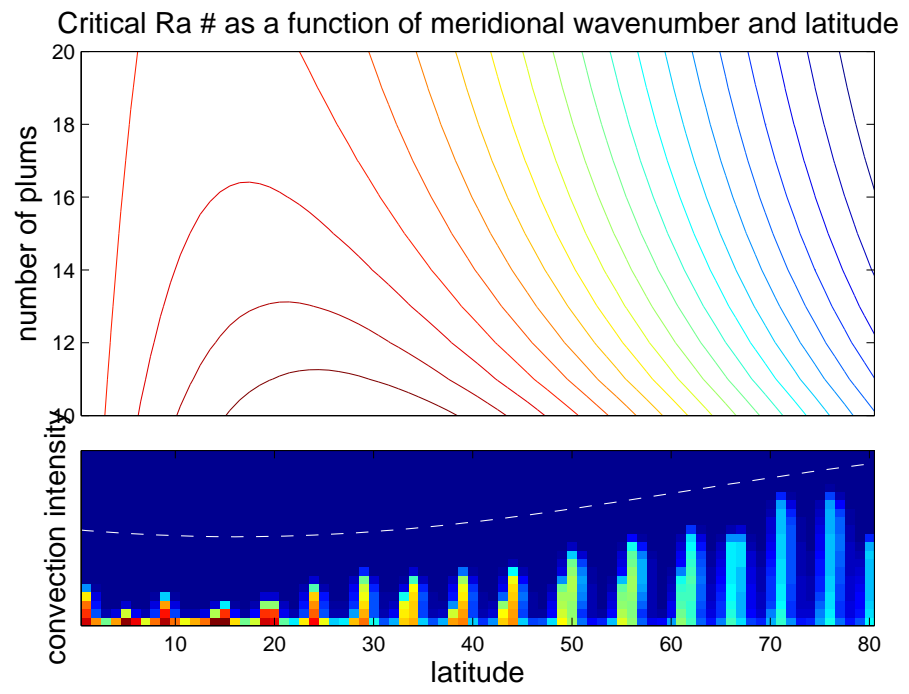

Figure 3.3: The critical Rayleigh number as function of latitude. (bottom) The color plot is the intensity of the convection at its onset, for a case where forcing is applied as a bottom flux. The dashed line is the inverse of the critical Rayleigh number (3.11), which matches the profile set by the outbreak of convection as a function of latitude. (top) The critical Rayleigh number as function of latitude and wavenumber. The level $l=17$ correspond to the dashed line in the bottom panel.

therefore that for a radius $r_{0}$ when

$$
\left(\frac{2 \Omega r_{0}^{2}}{\nu l^{3}}\right)^{2}>1
$$

the critical Rayleigh number decreases with latitude.

\subsection{From the $2 \mathrm{D}$ to the $3 \mathrm{D}$ Model}

Due to the natural axisymmetric appearance of thermally convecting rotating systems in nature, they have been initially studied for axisymmetric cases. Chandrasekhar (1961) showed that thermal convection in a rotating fluid for high Taylor numbers will form convection cells. Roberts (1968) was the first to show that linear asymmetric modes will be the most unstable in a spherical shell when forced internally by convection. In several studies Busse suggested that these modes are related to the jets seen on the outer planets and may lead to equatorial superrotation (e.g. Busse, 1970, 2002). Even on Earth's atmosphere, although a very different type of system, the 
statistically averaged flow appears axisymmetric. However, the mechanisms driving this flow do depend on the zonal asymmetries (Schneider, 2006).

Comparing our axisymmetric simulations to the 3D ones we find that the zonal asymmetries completely change the character of the circulation. The axisymmetric model is composed of mainly up-down motion along the direction of the axis of rotation with zonal velocities produced by divergences constrained by mass conservation of this convective flow. For rapid enough rotation the Taylor-Proudman theorem limits the motion. As a simple example we can think of the flow at the equator in the axisymmetric and Boussinesq case. At the equator the direction of the axis of rotation coincides with the latitudinal direction and therefore the Taylor-Proudman theorem implies that the meridional velocity is independent of the latitudinal direction. Since the velocity is non-divergent, then both derivatives independently become zero

$$
\frac{1}{r} \frac{\partial v}{\partial \theta}=\frac{\partial w}{\partial r}=0 .
$$

Then, since the boundary condition has no normal flow there can be no flow along the equatorial plane. Since the presence of convective plumes drives the flow away from a completely barotropic state, the Taylor-Proudman theorem does not completely apply even for the Boussinesq case and therefore some cross-equatorial flow does develop even in the axisymmetric model. However in the case of forcing only by a bottom boundary flux (as in section 3.1.2), we find there to be nearly no flow on the equatorial plane. A similar argument will hold for the anelastic case even though the mass divergence contains the mean density. It can be seen in Figure 3.4 that for both cases the equatorial region is fairly quiescent. In the 3D case, having the extra degree of freedom, the full 3D velocity divergence allows motion on the equatorial plane both in the zonal and radial directions even if the Taylor-Proudman constraint is fully applicable. In chapters 4-6 we discuss in detail the 3D solution, and in chapter 5 we show how this motion on the equatorial plane drives the equatorial superrotation. In Figure 3.4 we show the Anelastic and Boussinesq cases in 2D and the equivalent plots for the zonally symmetric flow in $3 \mathrm{D}$. The left panels are the zonal velocity and right panels are the meridional 2D streamfunction of the zonally averaged velocity. 

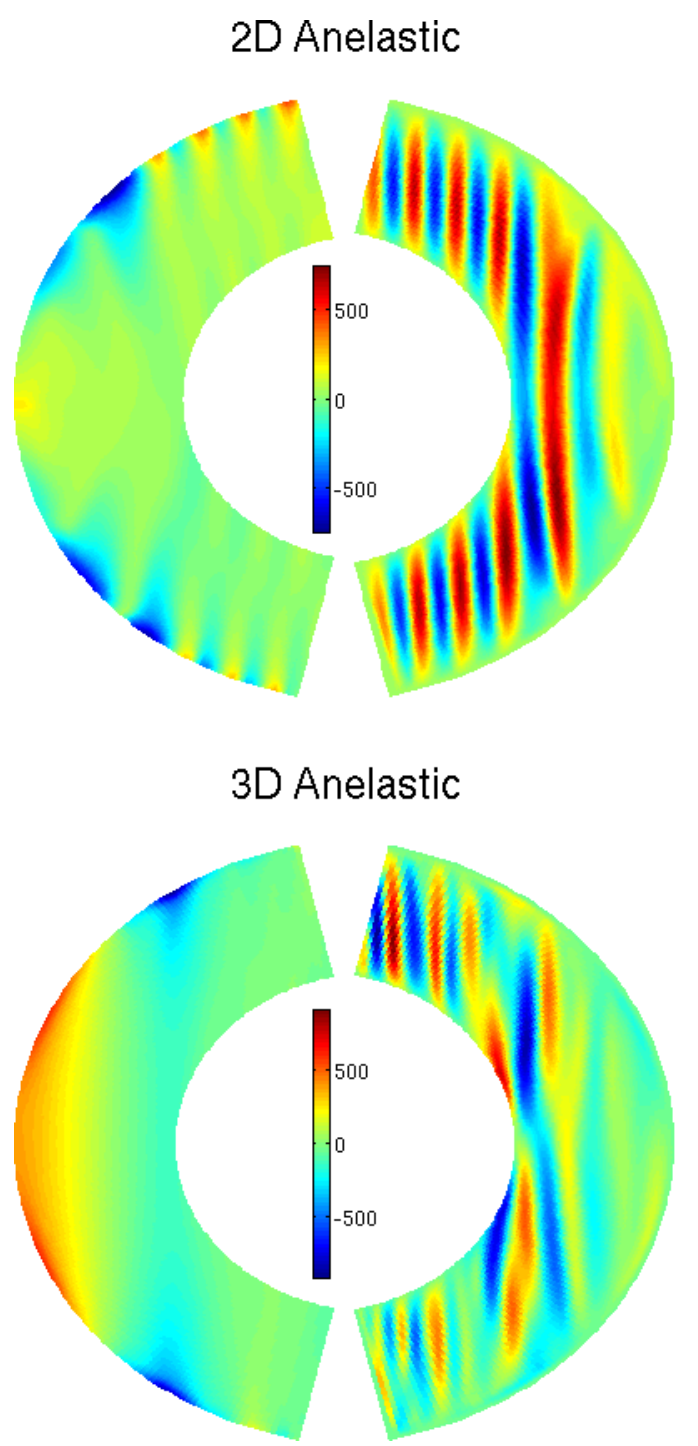
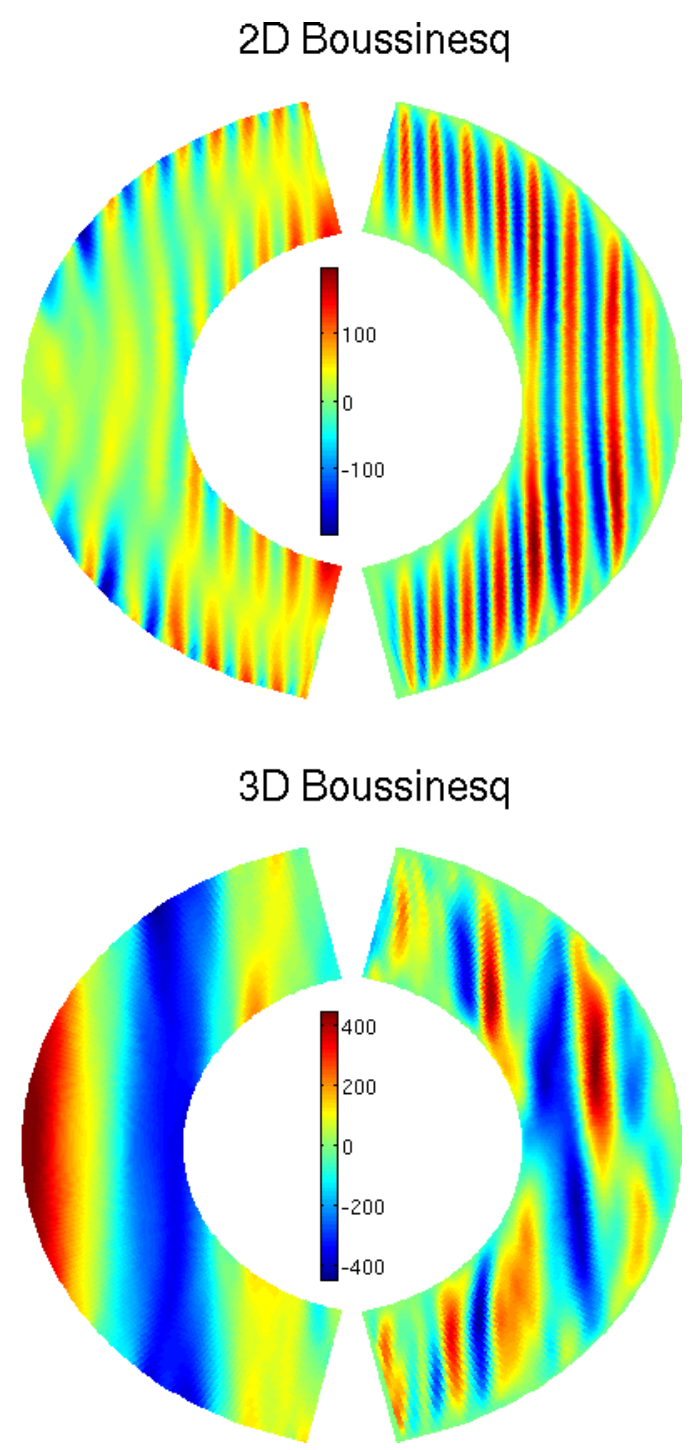

Figure 3.4: Comparing 2D and 3D Boussinesq and anelastic models. Left panels are zonal velocity (with $\mathrm{m} / \mathrm{s}$ values in the colorbar), and right panels are the $2 \mathrm{D}(r-\theta)$ streamfunction (zonally averaged fields for the 3D cases). 3D runs have parameters: $R a=1 E 7, E k=1.5 E-4, \operatorname{Pr}=10$, and 2D runs $R a=1 E 6, E k=4 E-4, \operatorname{Pr}=10$ (anelastic) and $R a=3 E 6, E k=1.5 E-4, \operatorname{Pr}=10$ (Boussinesq). 


\subsection{The 3D model}

In chapters 4-6 we discuss and analyze the 3D results. As a reference for the rest of this work in this section we present a series of plots which will be the baseline for future discussion. To describe the 3D spherical statistical steady state of the model, we present some of the basic fields in three orthogonal slices on the planet: a meridional (pole-to-pole) slice of the zonal mean flow, an equatorial $360^{\circ}$ slice around the planet (for some runs we have done only $90^{\circ}$ ), and slices on constant mean pressure surfaces.

The meridional extent of these runs has been from latitude $80^{\circ} \mathrm{N}$ to $80^{\circ} \mathrm{S}$. The choice of not extending the model to the pole was based on numerical convenience since the convergence of the grid at the pole will require more computation time. In addition we were more interested in the equatorial dynamics and therefore made this choice. The depth of the fluid layer was chosen for these runs at 0.55 the radius of the planet, which corresponds to approximately to 20 Mbar. In chapter 2 we have shown that beyond about $100 \mathrm{kbar}$ the thermodynamics become different than an ideal gas, and therefore we are well into that regime. Most previous models of convection in a deep shell put the bottom boundary at a higher level. However, it has not been clear how much that choice influences the results (in particular the extent of the superrotation). One of the goals of this work is to study the dynamics of a deep system and therefore we deliberately push the bottom boundary deep even beyond what is generally accepted. In section 6.1 we study the dependence of the dynamics on the location of the bottom boundary using a series of runs ranging from a thin spherical shell to a full 3D sphere. We use slip boundary conditions on the bottom and side boundaries, and a free surface on top.

All runs we present here have a $1^{\circ}$ resolution and a factor of 1.33 in pressure between each vertical level, with a total of 120 vertical grid points, giving a total of $160 \times 360 \times 120$ grid points. Because of the convection, the numerical time step is small ( 5 seconds) and the runs typically require at least $5 E 5$ time steps to reach a statistical steady state, beginning with a zero mean flow initial condition and small random noise. We run typically on 16 parallel processors and computation time for such a configuration is about 6 weeks. We found that using only part of the sphere (typically $\frac{1}{4}$ of the sphere zonally) with periodic longitudinal boundary conditions does not affect the results much, and allows cutting computational time by a factor of 4 . Some of the runs we show therefore will be of a slice of a fourth of a sphere. 
Mainly for presentation purposes throughout this work we use the meridional and zonal components of the vector streamfunction $\Psi$ to describe the flow on a $2 \mathrm{D}$ slice. Since the quantity $\bar{\rho} \mathbf{u}$ has zero divergence we can define this streamfunction as

$$
\nabla \times \Psi \equiv \bar{\rho} \mathbf{u}
$$

where $\Psi$ is a $3 \mathrm{D}$ vector. In component form this gives

$$
\begin{aligned}
\frac{1}{r \cos \theta}\left[\frac{\partial\left(\Psi_{\phi} \cos \theta\right)}{\partial \theta}-\frac{\partial \Psi_{\theta}}{\partial \phi}\right] & =\bar{\rho} w \\
\frac{1}{r}\left[\frac{1}{\cos \theta} \frac{\partial \Psi_{r}}{\partial \phi}-\frac{\partial\left(r \Psi_{\phi}\right)}{\partial r}\right] & =\bar{\rho} v \\
\frac{1}{r}\left[\frac{\partial\left(r \Psi_{\theta}\right)}{\partial r}-\frac{\partial \Psi_{\theta}}{\partial \theta}\right] & =\bar{\rho} u .
\end{aligned}
$$

Due to the symmetry along the axis of rotation, on the equator we assume the changes along the axis of rotation (which coincide with the $\theta$ direction along the equatorial plane) are small, and then can neglect the terms containing changes in the $\theta$ direction for the equatorial plane. Then, we can integrate either (3.15) or (3.17) to find $\Psi_{\theta}$. We refer to this meridional component as the equatorial streamfunction. In Figure 3.11 we show velocity vectors superimposed on the equatorial streamfunction showing that integrating from either (3.15) or (3.17) is consistent. As one moves away from the equatorial plane this approximation becomes less accurate. For the $\Psi_{r}$ component we find that since the motion is $3 \mathrm{D}$, we can not describe $\Psi_{r}$ as a $2 \mathrm{D}$ field. The zonally averaged values are presented as the averaged meridional streamfunction $\Psi_{\phi}$.

We show in this section results from two runs which have identical parameters except for the Rayleigh number. Our goal is to run the model in a regime which is as turbulent as the numerics will allow, and therefore have a Rayleigh number which is as high as we can afford (also depends on grid and time step), though it is harder to identify the physical processes in those runs. Therefore in section 5.1 we study in detail a run with a low Rayleigh number which allows easier analysis of processes. The runs we present in this section have Ra numbers of $5 E 7$ and $3 E 6$ which we will refer to as the high and moderate Rayleigh number runs respectively. We begin with the high Rayleigh number run, and in Figures 3.5, 3.6, 3.7, and look at slices on surfaces of constant mean pressure (depth) which are roughly at the top surface, 0.86 , and 0.59 of the radius respectively (1bar, 1 Mbar and $10 \mathrm{Mbar}$ ). The fields are 
averaged over a period of 1 day. For each surface we look at the three components of velocity, density anomaly, entropy anomaly, and vorticity (top surface) or pressure anomaly (bottom two). Velocity fields show the effect of the mean density with smaller velocities in the interior and the superrotation at the equator with a weaker Hadley cell in the meridional direction. The thermodynamic fields show how density is strongly affected by pressure in the higher levels while becoming more dependent on entropy in the lower levels. We discuss this issues in section 4.3.2. Figure 3.8 shows the corresponding fields for the same high Rayleigh number run on the equatorial plane, including the equatorial 2D streamfunction (flow in the $r-\phi$ plane), showing cyclonic eddies on the equatorial plane. The zonally averaged meridional slices are similar to the moderate Rayleigh number runs (only with stronger velocities), and therefore we show them for that run only.

For the moderate Rayleigh number runs we look both at the instantaneous fields, and at the time averaged fields averaged over 12 days. Beginning with the instantaneous fields (snapshots) in Figures 3.9, 3.10, 3.11 we show the zonally mean fields on the meridional plane, the surface at 1 bar, and the equatorial plane respectively. Then we show the same slices for the 1bar surface and the equatorial plane without repeating the meridional plane that is quite similar to the instantaneous fields because of the zonal mean. In the following chapters we discuss the features of these runs in more detail and discuss their dynamics. Figures $3.5-3.13$ follow below. 

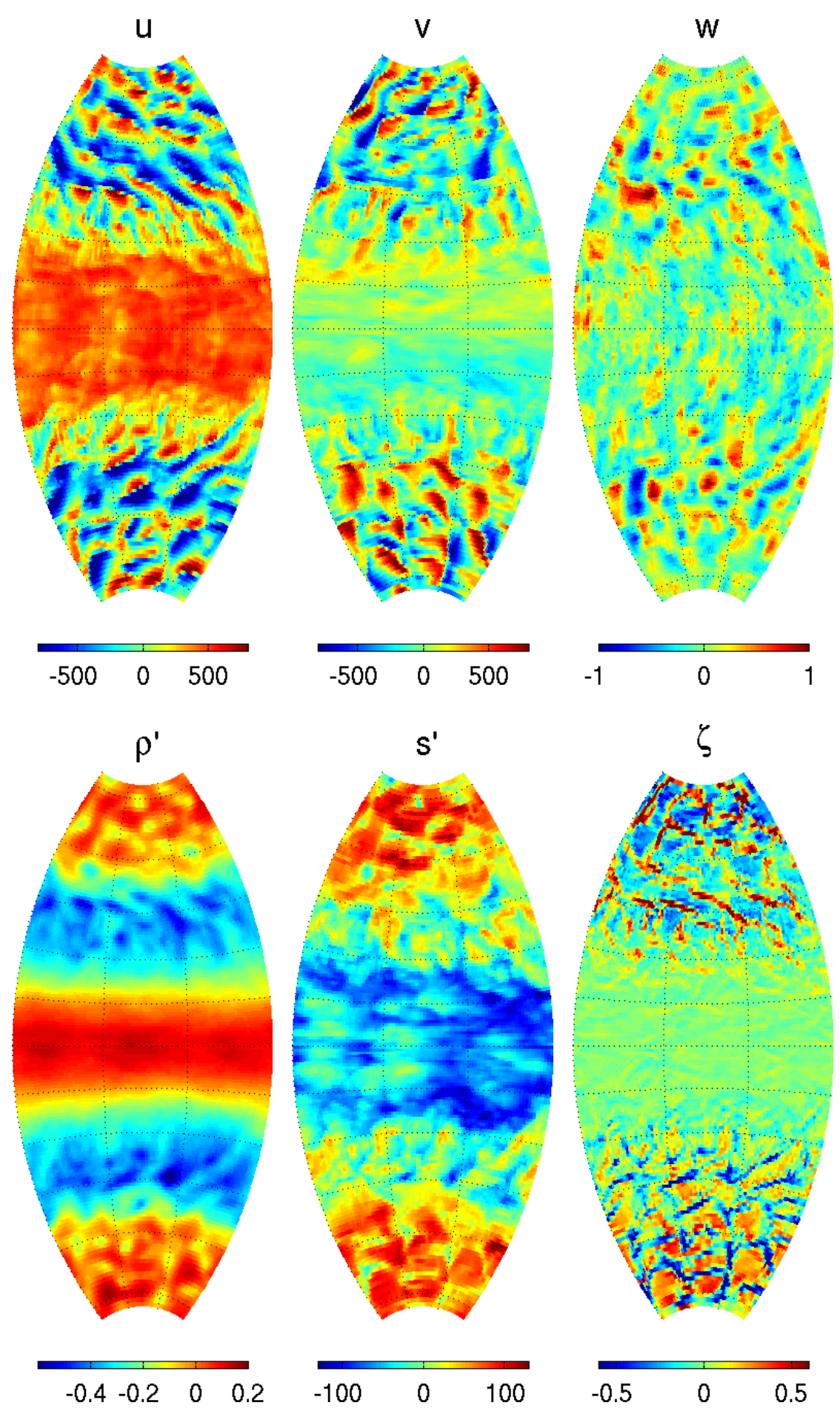

Figure 3.5: 1 bar surface fields averaged over 1 day for a high Rayleigh number run: $R a=5 E 7, E k=1.5 E-4, \operatorname{Pr}=10$. upper left: zonal velocity $[\mathrm{m} / \mathrm{s}]$; upper middle: meridional velocity $[\mathrm{m} / \mathrm{s}]$; upper right: vertical velocity $[\mathrm{m} / \mathrm{s}]$; lower left: density anomaly $\left[\mathrm{Kg} \mathrm{m}^{-3}\right]$; lower middle: converted entropy (see Appendix A) anomaly $[K]$; lower right: vertical vorticity $\left[10^{-3} \mathrm{~s}^{-1}\right]$. 

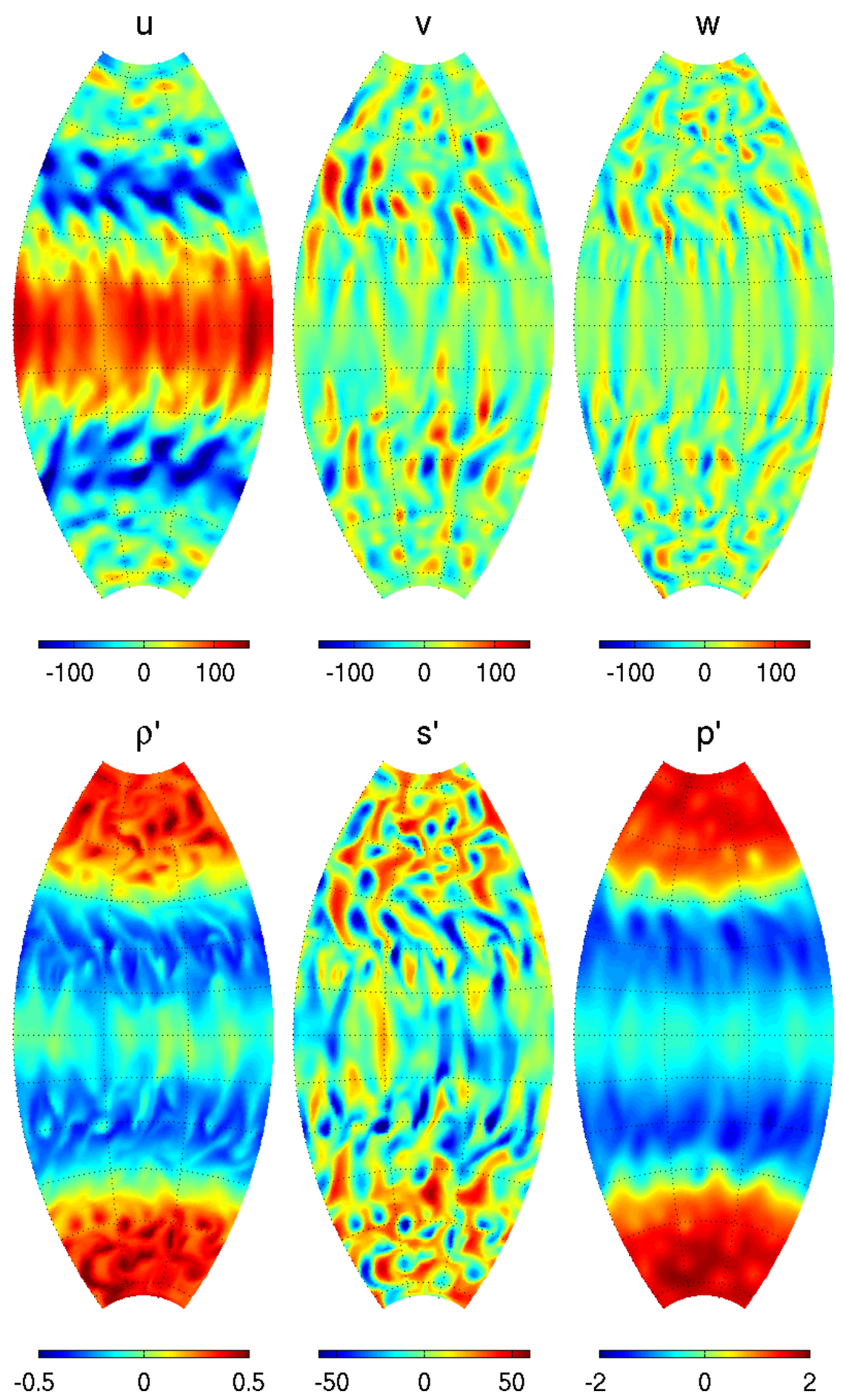

Figure 3.6: 1 Mbar surface fields ( 0.86 of the radius) averaged over 1 day for a high Rayleigh number run: $R a=5 E 7, E k=1.5 E-4, P r=10$. upper left: zonal velocity $[\mathrm{m} / \mathrm{s}]$; upper middle: meridional velocity $[\mathrm{m} / \mathrm{s}]$; upper right: vertical velocity $[\mathrm{m} / \mathrm{s}]$; lower left: density anomaly $\left[\mathrm{Kg} \mathrm{m}^{-3}\right]$; lower middle: converted entropy anomaly $[K]$; lower right: pressure $[k b a r]$. 

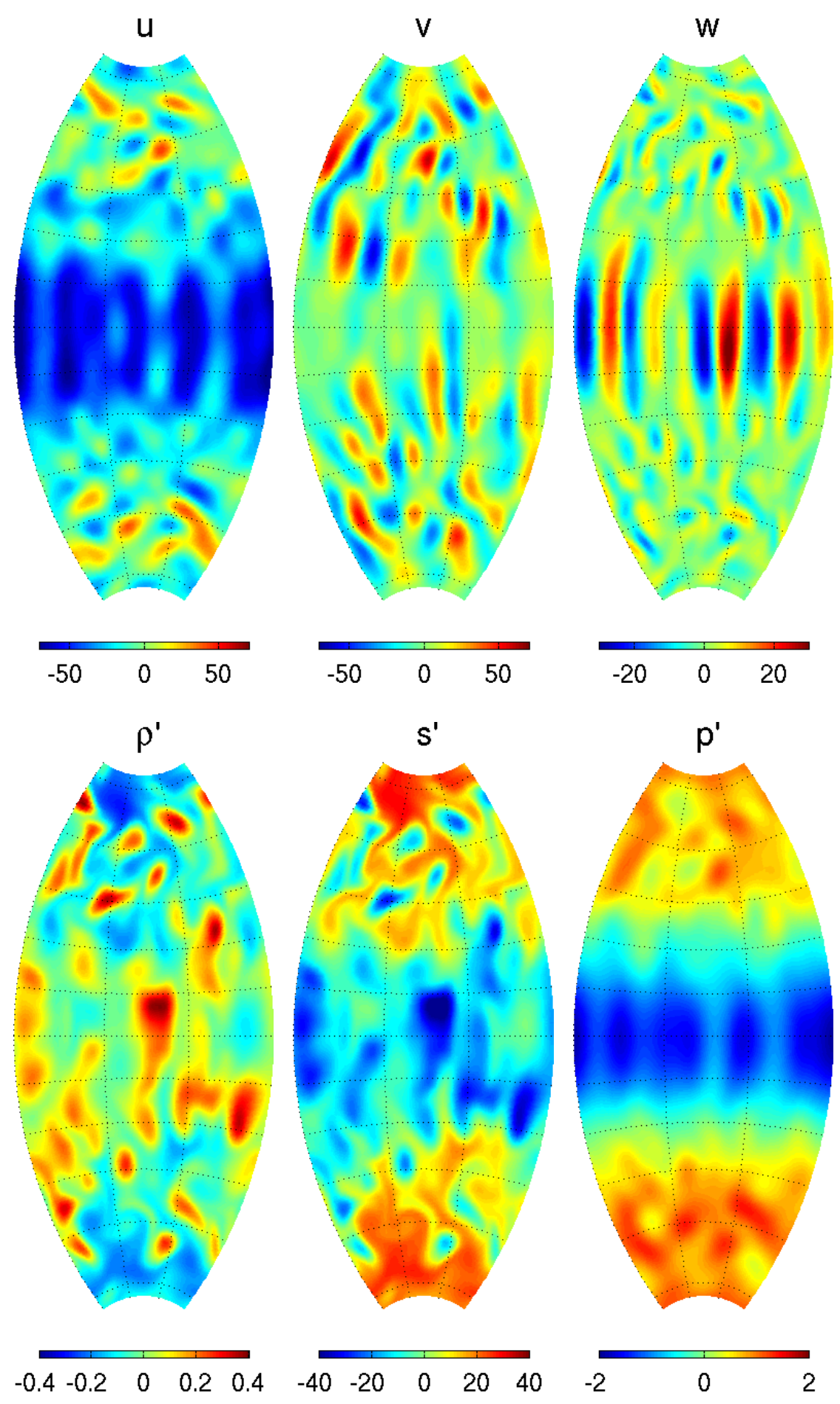

Figure 3.7: 10 Mbar surface fields (0.59 of the radius) averaged over 1 day for a high Rayleigh number run: $R a=5 E 7, E k=1.5 E-4, \operatorname{Pr}=10$. upper left: zonal velocity $[\mathrm{m} / \mathrm{s}]$; upper middle: meridional velocity $[\mathrm{m} / \mathrm{s}]$; upper right: vertical velocity $[\mathrm{m} / \mathrm{s}]$; lower left: density anomaly $\left[\mathrm{Kg} \mathrm{m}^{-3}\right]$; lower middle: converted entropy anomaly $[K]$; lower right: pressure [kbar]. 

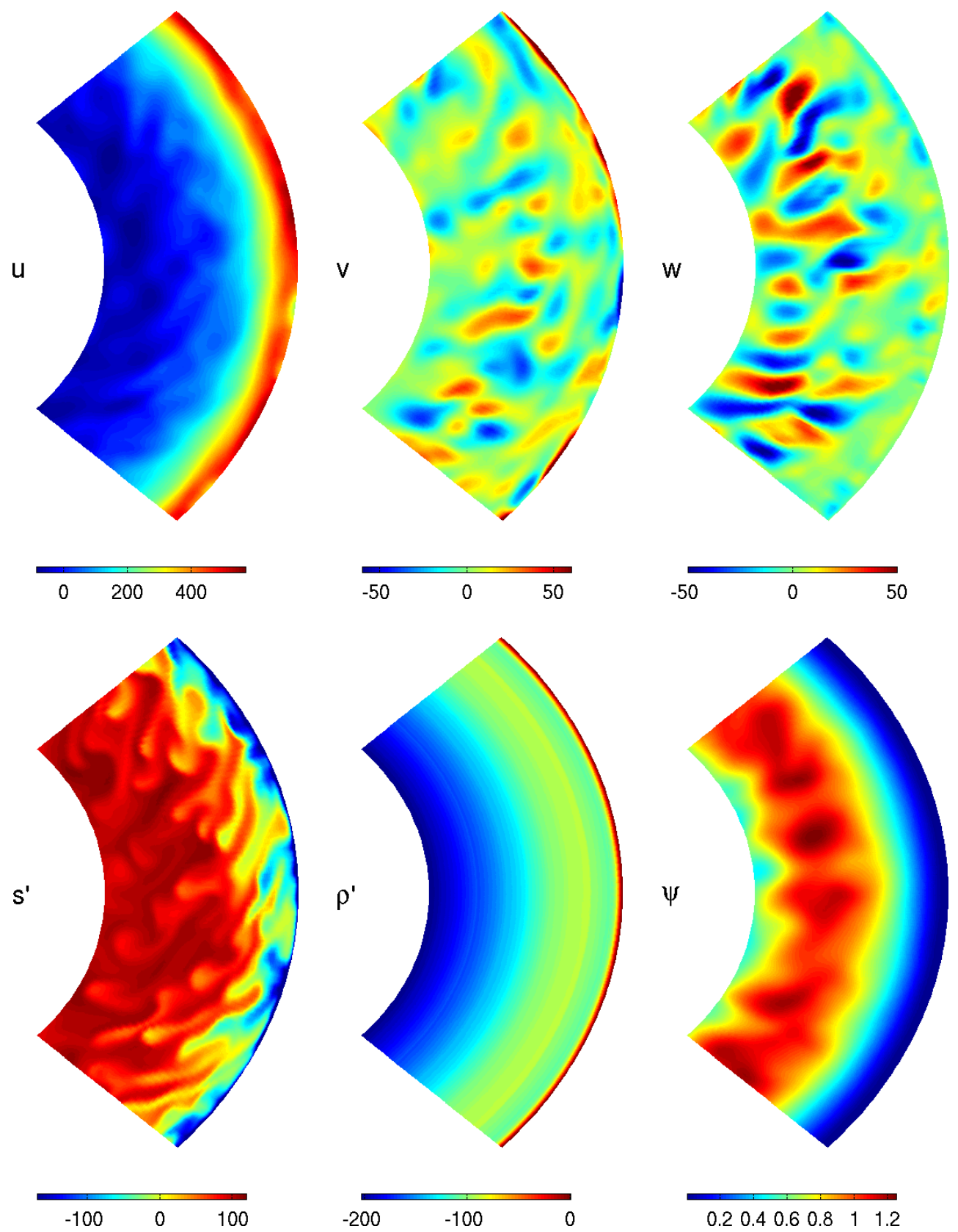

Figure 3.8: Equatorial plane slices averaged over 1 day for a high Rayleigh number run: $R a=5 E 7, E k=1.5 E-4, \operatorname{Pr}=10$. upper left: zonal velocity $[\mathrm{m} / \mathrm{s}]$; upper middle: meridional velocity $[\mathrm{m} / \mathrm{s}]$; upper right: vertical velocity $[\mathrm{m} / \mathrm{s}]$; lower left: converted entropy anomaly $[K]$; lower middle: density anomaly $\left[\mathrm{Kg} \mathrm{m}^{-3}\right]$; lower right: equatorial streamfunction $[1 / s]$. 

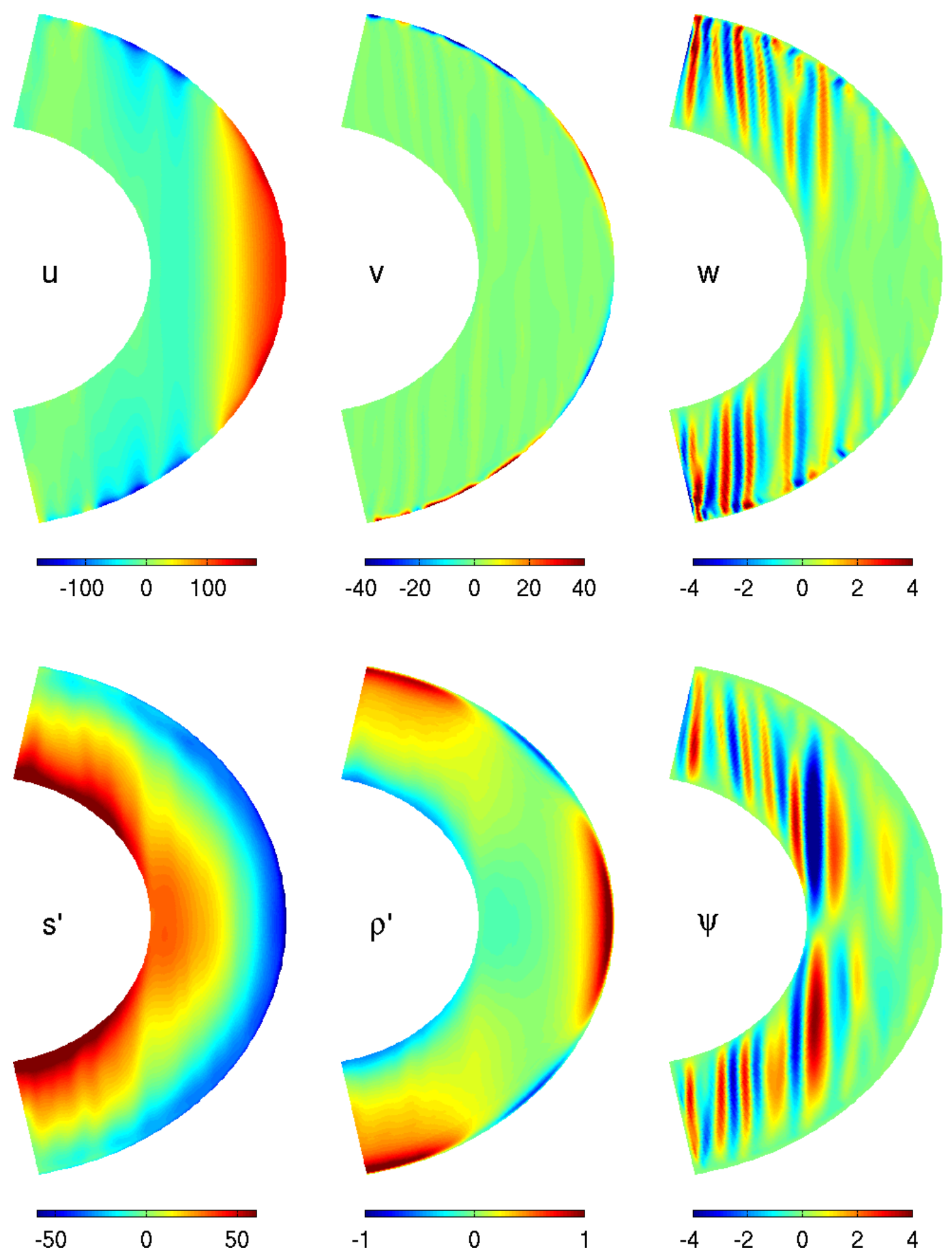

Figure 3.9: Snapshots of zonally averaged fields on a meridional section for a run with a moderate Rayleigh number: $R a=3 E 6, E k=1.5 E-4, \operatorname{Pr}=10$. Upper left: zonal velocity $[\mathrm{m} / \mathrm{s}]$; upper middle: meridional velocity $[\mathrm{m} / \mathrm{s}]$; upper right: vertical velocity $[\mathrm{m} / \mathrm{s}]$; lower left: converted entropy anomaly $[K]$; lower middle: density anomaly $\left[\mathrm{Kg} \mathrm{m}^{-3}\right]$; lower right: 2D meridional streamfunction $[1 / \mathrm{s}]$. 

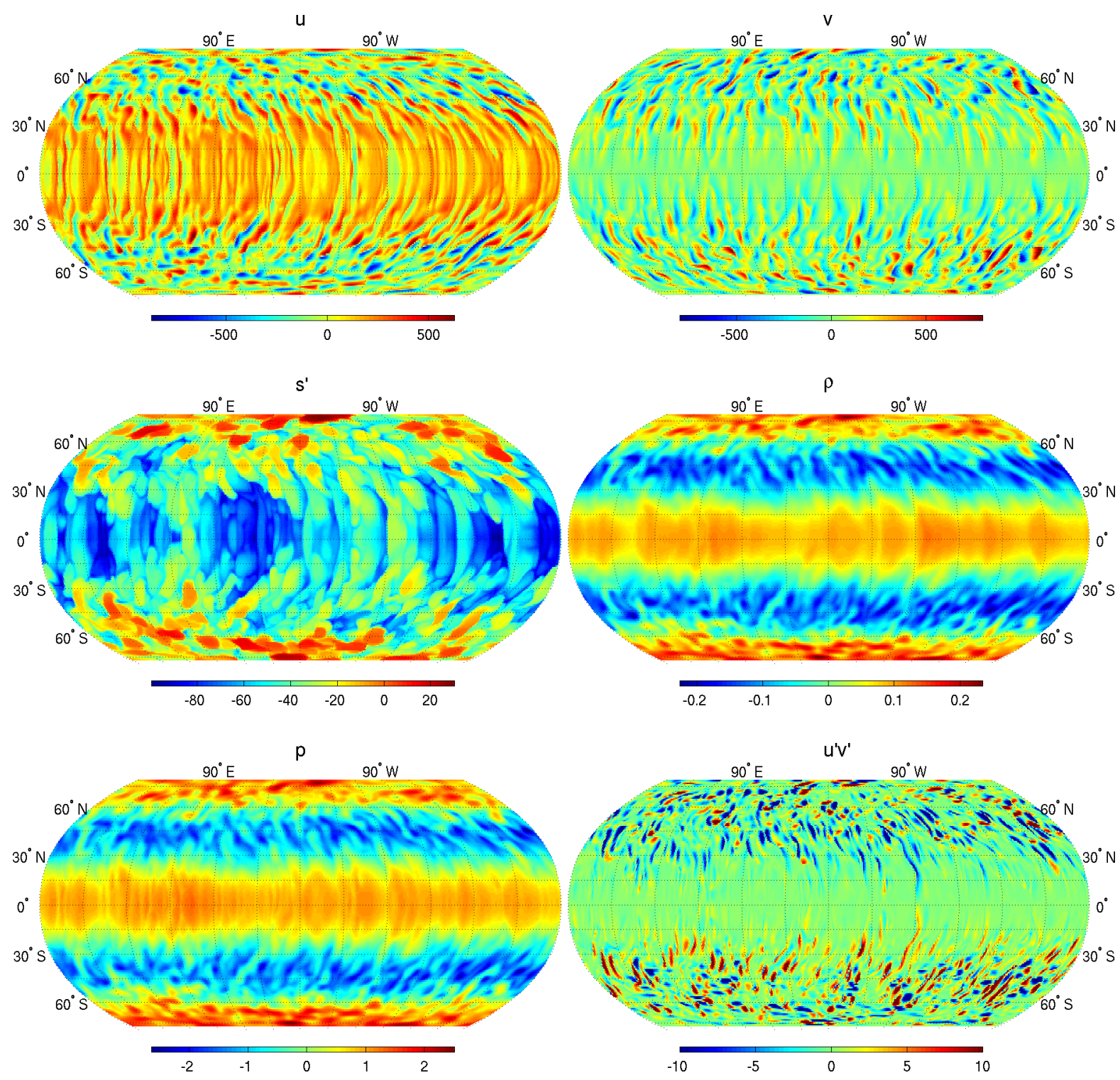

Figure 3.10: Snapshots of fields at the 1 bar surface for a run with a moderate Rayleigh number. $R a=3 E 6, E k=1.5 E-4, \operatorname{Pr}=10$. Upper left: zonal velocity $[\mathrm{m} / \mathrm{s}]$; upper right: meridional velocity $[\mathrm{m} / \mathrm{s}]$; middle left: converted entropy anomaly $[K]$; middle right: density anomaly $\left[\mathrm{Kg} \mathrm{m}^{-3}\right]$; lower left: pressure anomaly [bar]; lower right: momentum flux $\left[10^{-4} \mathrm{~m}^{2} / \mathrm{s}^{2}\right]$. 

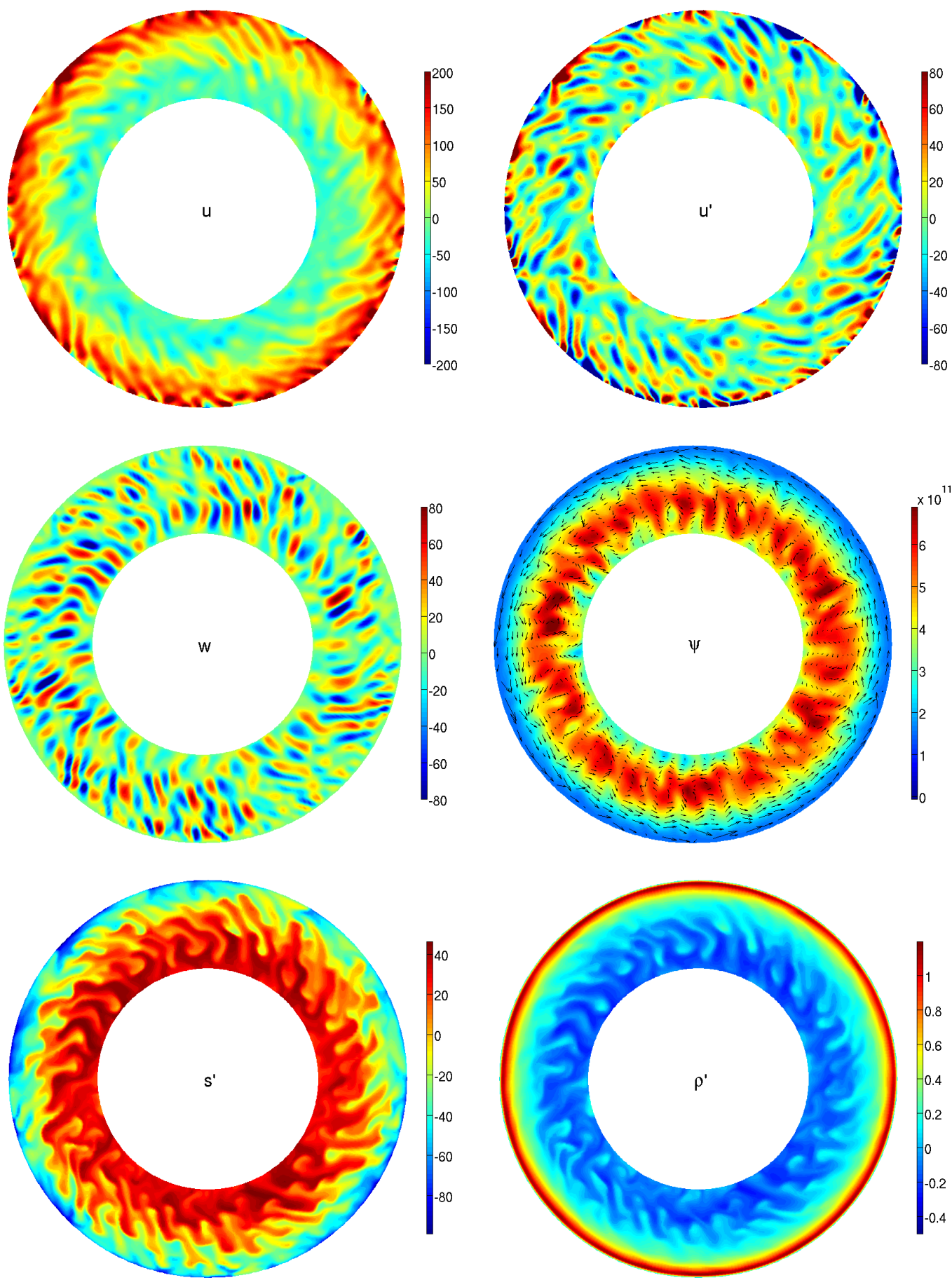

Figure 3.11: Snapshots of fields on an equatorial section for a run with a moderate Rayleigh number: $R a=3 E 6, E k=1.5 E-4, \operatorname{Pr}=10$. Upper left: zonal velocity $[\mathrm{m} / \mathrm{s}]$; upper right: zonal velocity anomaly (subtracting the zonal mean from the zonal velocity) $[\mathrm{m} / \mathrm{s}]$; middle left: vertical velocity $[\mathrm{m} / \mathrm{s}]$; middle right: $2 \mathrm{D}$ equatorial streamfunction $[1 / s]$; bottom right: converted entropy anomaly $[K]$; density anomaly $\left[\mathrm{Kg} \mathrm{m}^{-3}\right]$. 

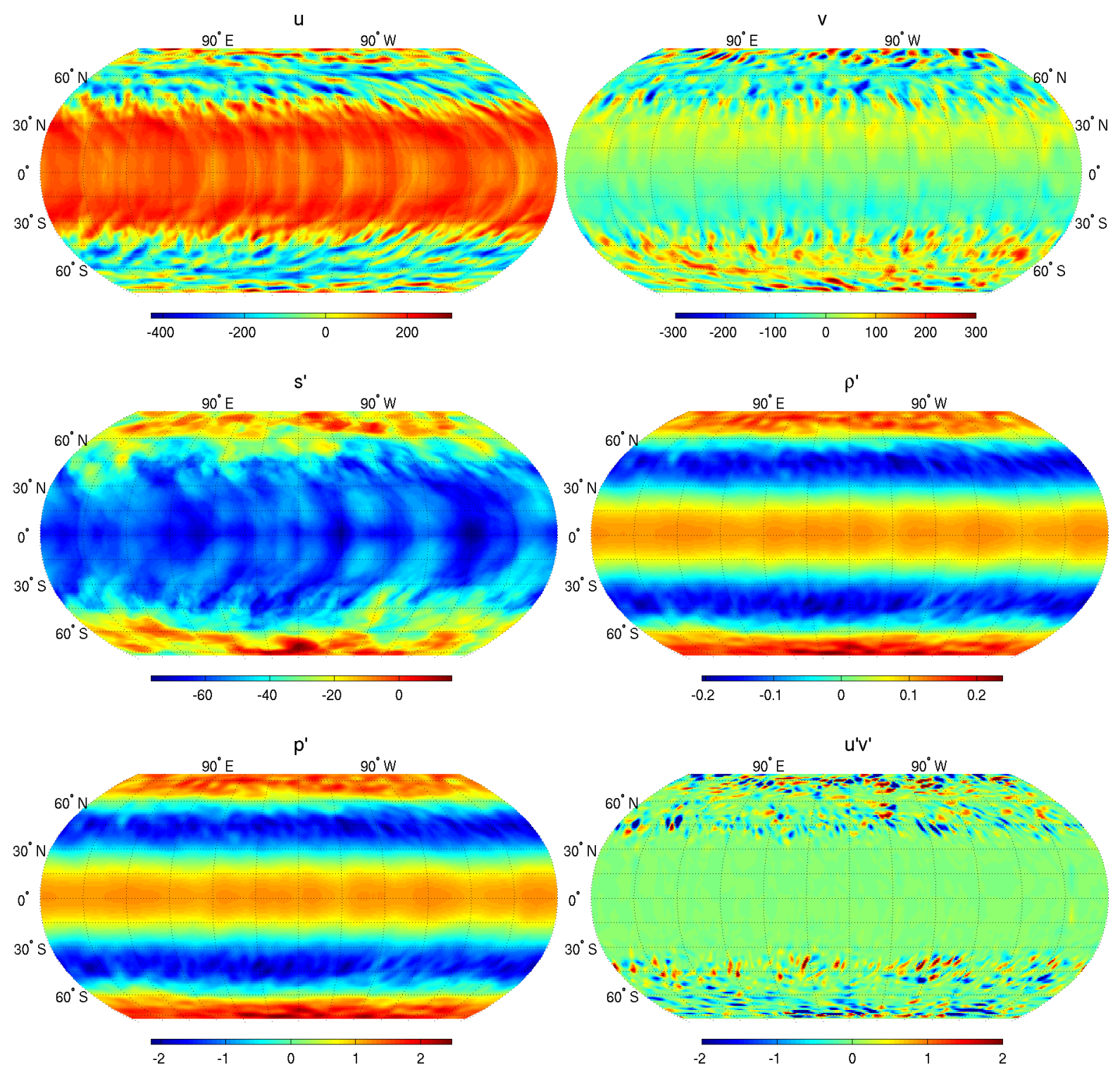

Figure 3.12: 1 bar surface for a run with a moderate Rayleigh number time averaged over 12 days. $R a=3 E 6, E k=1.5 E-4, \operatorname{Pr}=10$. Upper left: zonal velocity $[\mathrm{m} / \mathrm{s}]$; upper right: meridional velocity $[\mathrm{m} / \mathrm{s}]$; middle left: converted entropy anomaly $[K]$; middle right: density anomaly $\left[\mathrm{Kg} \mathrm{m}^{-3}\right]$; lower left: pressure anomaly [bar]; lower right: momentum flux $\left[10^{-4} \mathrm{~m}^{2} / \mathrm{s}^{2}\right]$. 

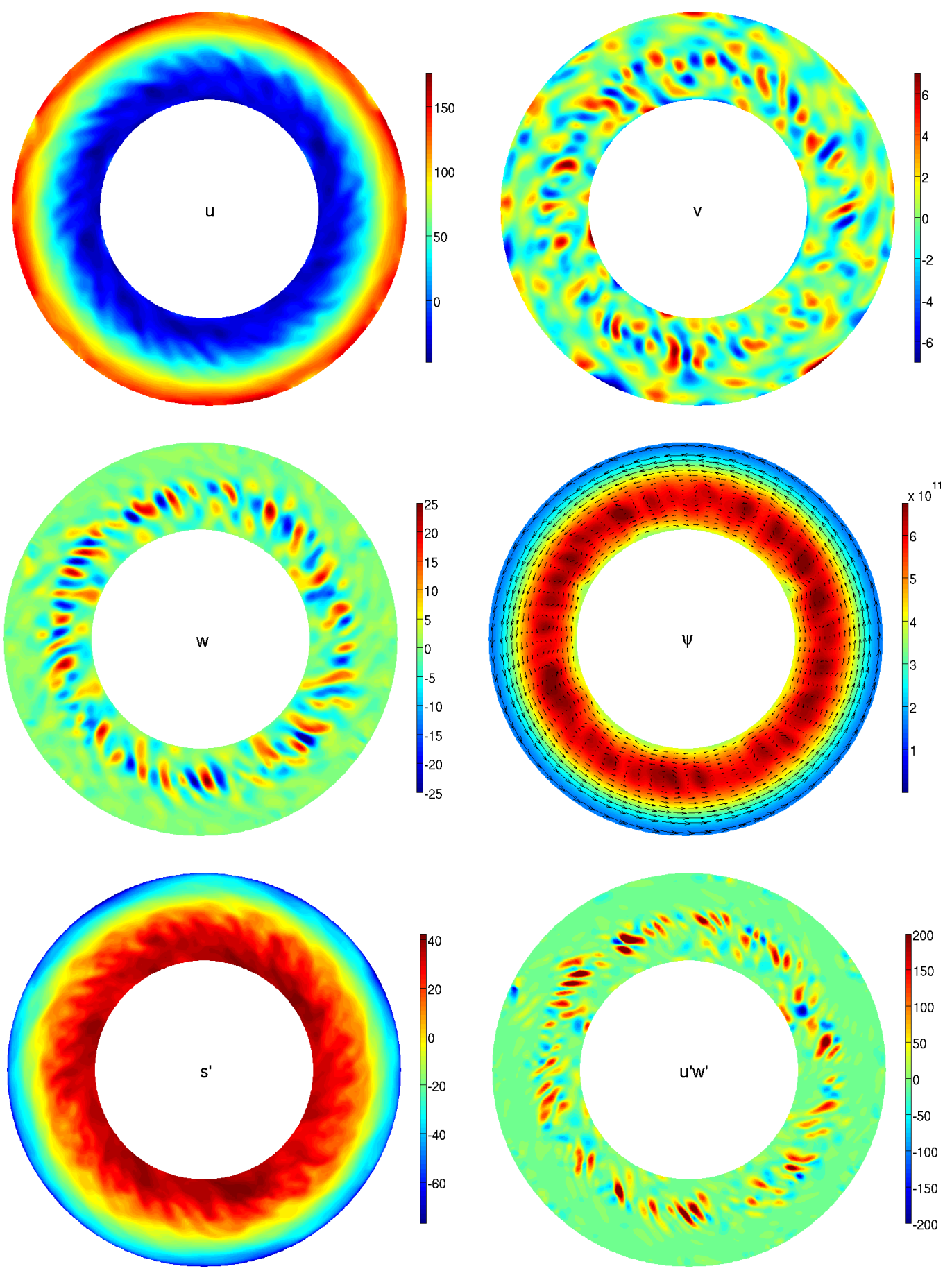

Figure 3.13: Equatorial sections for a run with a moderate Rayleigh number time averaged over 12 days: $R a=3 E 6, E k=1.5 E-4, \operatorname{Pr}=10$. Upper left: zonal velocity $[\mathrm{m} / \mathrm{s}]$; upper right: meridional velocity $[\mathrm{m} / \mathrm{s}]$; middle left: vertical velocity $[\mathrm{m} / \mathrm{s}]$; middle right: 2D equatorial streamfunction $[1 / \mathrm{s}]$; bottom right: converted entropy anomaly $[K]$; vertical momentum flux $\left[\mathrm{m}^{2} / \mathrm{s}^{2}\right]$. 


\section{Chapter 4}

\section{Basic Balances and the Vertical Wind Structure}

\subsection{Introduction}

One of the most fundamental questions regarding the atmospheres of the gas giant planets is how deep are the strong winds which are observed in their atmospheres. The only direct observation is from the Galileo probe, which showed an increase in zonal velocity from $80 \mathrm{~m} / \mathrm{s}$ to $160 \mathrm{~m} / \mathrm{s}$ down to the 4 bar level, and then a constant wind speed for as far down as the data could be retrieved (the 24 bar level), (Atkinson et al., 1996). Beyond the problem of having only a single measurement profile, the probe entered a "hot-spot" which may not be a good representation of the general flow (Bagenal et al., 2004). Other observational evidence for the deep flow comes from the fact that the heat emission on both Jupiter and Saturn has a nearly uniform meridional structure (Ingersoll, 1976; Hanel et al., 1981, 1983), suggesting deep transfer of heat (Ingersoll and Porco, 1978). One of the main goals of the JUNO mission is to put constraints on the depth of the jets via gravity measurements (section 8.2.2) . Recently, Liu (2006) put theoretical constraints on the possible extent of deep flows based on the ohmic dissipation created by the zonal flows in an electrically conducting fluid by the magnetic field. They suggest that if the zonal flows in the interior would be as strong as they are on the surface, and the magnetic field can also be deduced by the surface values, then the zonal winds could not penetrate more than 0.95 and 0.87 of the radius on Jupiter and Saturn respectively. In this study we do not include the 
effect of the magnetic field; however we show that even without the magnetic field acting to dissipate the flow in the interior, we do not expect to find interior velocities as large as the atmospheric ones, based only on the big increase in density between the outer atmosphere and the interior.

In this chapter we attempt to address the question of the deep velocities using our numerical model. Previous models could not address this issue since they were either shallow type models (e.g. Cho and Polvani, 1996; Showman et al., 2006) or deep models that were restricted to the Boussinesq approximation (Sun et al., 1993; Zhang and Schubert, 1996, 1997; Aurnou and Olson, 2001). For example Heimpel et al. (2005) and Heimpel and Aurnou (2007) show superrotating equatorial zonal flow, with higher latitude meridionally confined jets in a Boussinesq model which goes down to 0.9 of the planetary radius. The zonal velocities persist throughout the depth of the planet, and the meridional extent of the equatorial superrotating jet depends on the location of the bottom boundary. Clearly for addressing the baroclinic structure of the zonal winds we want allow density variations over the depth of the planet. Using both an anelastic model and a suitable equation of state allows us to address this issue more thoroughly. We try to decouple our results from the choice of the location of the bottom boundary and therefore push it deep below what is believed to be the boundary of the molecular fluid (we experiment with the bottom boundary location in section 6.1 ). We find the compressibility effects very important in understanding the vertical wind structure.

As discussed in the introduction, based on emission measurements and on 1D radiative theoretical models it is believed that the deep atmosphere is in a convective state (Guillot, 2005). A common assumption is that if the interior is convective it is close to a purely barotropic state. This is based on the assumption that convection causes uniform mixing limiting the density variations across pressure surfaces. We note two things: First convection tends to form plumes meaning that even if the atmosphere is driven by strong convection since the regions of strong upwelling plumes tend to be very localized (Lindzen, 1977), much of the atmosphere may be slightly stably stratified with small regions of convectively unstable plumes, and the atmosphere can still have horizontal density gradients. Second, the density anomalies are not just a function of entropy or heat anomalies, but also in an anelastic system are affected by the compressible effects, thus giving a significant baroclinic contribution.

In this chapter we begin by looking at the basic balances showing that to first or- 
der the motion is geostrophic and hydrostatic. Then we show how the thermal wind relation is revised when considering a deep atmosphere rather than one restricted to a spherical shell. Incorporating the anelastic approximation the vorticity equation highlights the importance of the baroclinic contributions, which are not small for a compressible gas. In the barotropic limit the system will still give the TaylorProudman constraint, but this is a more specific case than it appears for a Boussinesq fluid. We show that anelastic models must have density depending on two thermodynamic variables and otherwise can be misleading. We show how convection drives the system away from a barotropic state, and thus away from the Taylor-Proudman constraint. The convectively driven flow in steady state is in a state in between having Taylor columns, with the zonal velocity being constant along the direction of the rotation axis, to constant momentum $(\bar{\rho} u)$ along this direction. The baroclinic contributions therefore set the vertical shear, and in section 4.7 we proceed to parametrize the shear of the zonal flow using scaling arguments. We show the details of the interior circulation including the formation of large scale columnar structures which have been suggested in qualitative studies (Busse, 1976). These columnar structures surround the interior core and have vorticity in the same sense as the mean shear. We analyze the angular momentum and heat flux budgets and show the roles of eddy and mean fluxes in driving the circulation. We find that the zonal asymmetries and angular momentum eddy fluxes play an important role in transporting angular momentum to the equator and forming the equatorial superrotating zonal flows.

\subsection{Basic Balances}

Given the set of model equations (2.7), (2.8), (2.9), (2.10), (2.28) and (2.47), and the solutions presented in section 3.3 we begin by looking at the leading order balances in these solutions. These balances are important for understanding the key physical mechanisms in the dynamics and for further analysis when developing theories with higher order expansions. Beginning with the zonal momentum balance, for small Rossby and Ekman numbers the leading order terms in the momentum equations 

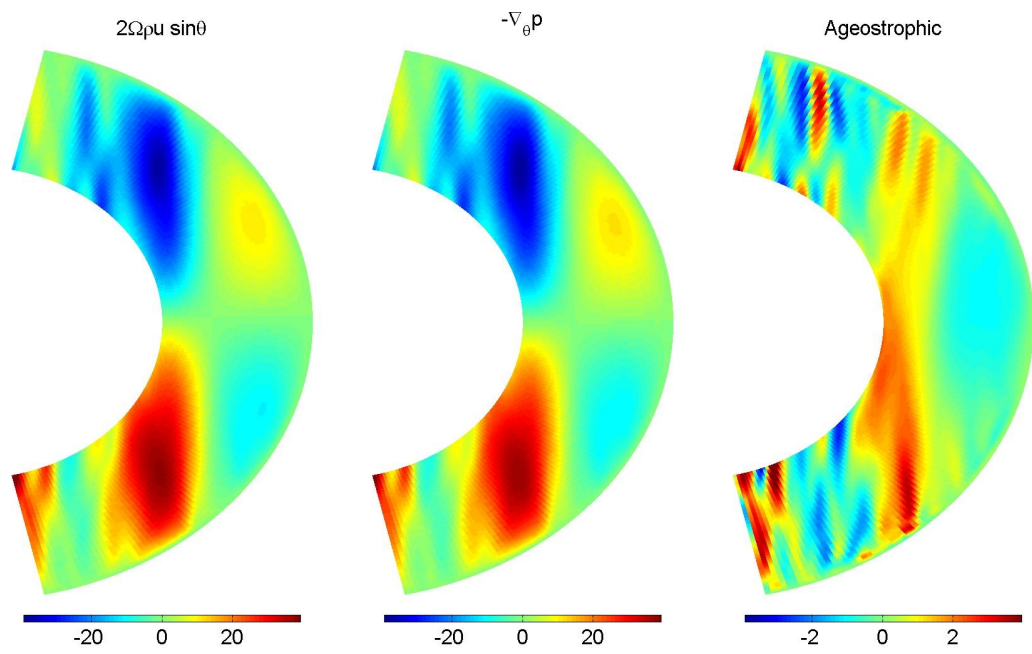

Figure 4.1: Geostrophic balance: the two plots on the left show the geostrophic balance for the zonally averaged fields (meridional section) and the difference between them is shown on the right.

(2.8-2.10) give

$$
\begin{aligned}
-2 \Omega \sin \theta v+2 \Omega \cos \theta w & =-\frac{1}{\bar{\rho} r \cos \theta} \frac{\partial p^{\prime}}{\partial \phi} \\
2 \Omega \sin \theta u & =-\frac{1}{\bar{\rho} r} \frac{\partial p^{\prime}}{\partial \theta} \\
-2 \Omega \cos \theta u & =-\frac{1}{\bar{\rho}} \frac{\partial p^{\prime}}{\partial r}-\frac{\rho^{\prime}}{\bar{\rho}} g
\end{aligned}
$$

where all variables and coordinates are the same as defined in chapter 2. Density and pressure have been expanded as in (2.1 and 2.2) to a mean horizontally independent hydrostatic part and an anomaly. Note that we are using the standard form of the vertical momentum equation and not the equivalent anelastic form with the revised gravity term as in (2.26). As discussed in section 2.2 in the deep system, apriori all four Coriolis terms contribute to the geostrophic balance. Here we show that indeed this is the case. The numerical results presented here are from 3D runs at a $1^{\circ}$ resolution and 120 vertical levels extending to 0.55 the radius of the planet. The pressure variation is from 1 bar in the upper level to 12 Mbars in the interior with a pressure increase of ratio 1.33 between vertical levels. Rayleigh, Prandtl and Ekman numbers as defined in $(2.53)$ are $5 E 7,10$ and $1.5 E-4$ respectively. In Figure 4.1 we show that to the first order the flow is in geostrophic balance; thus the pressure 

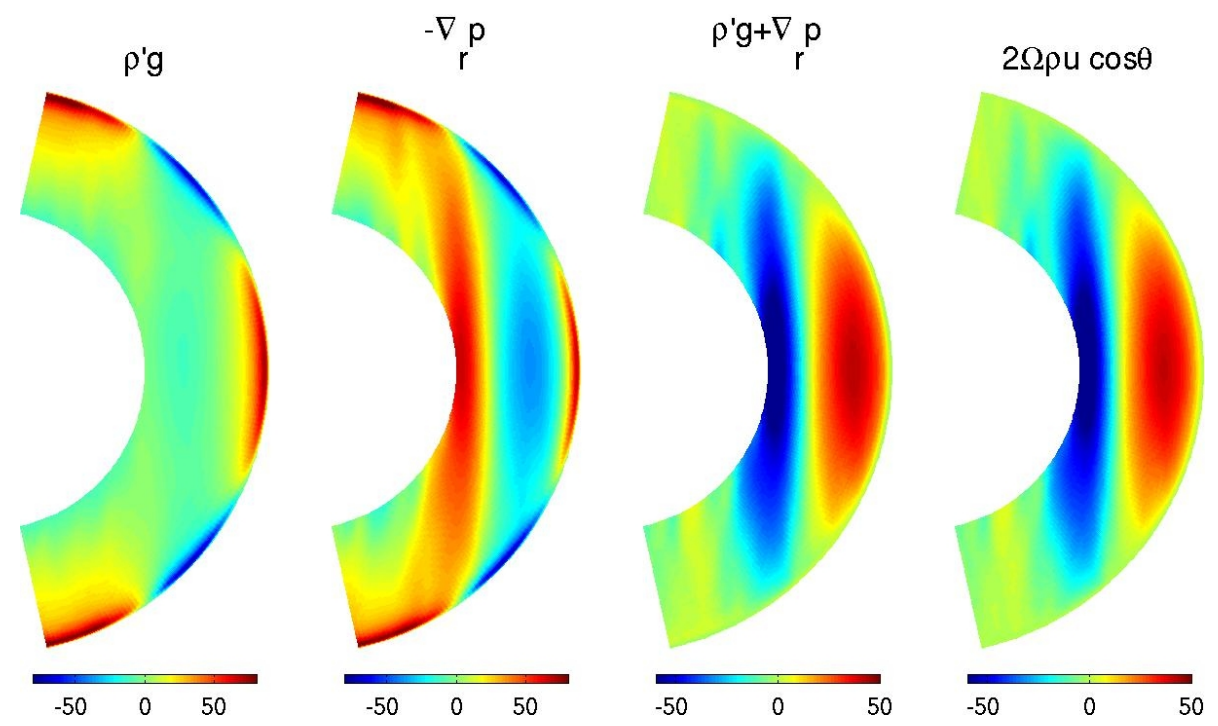

Figure 4.2: Hydrostatic balance: left: buoyancy term; middle-left: radial pressure gradient; middle-right: difference between the panels on the right; right: vertical Coriolis term

gradients are in balance with the Coriolis term in equation 4.2. In (4.1) the zonally averaged Coriolis terms balance each other. The ageostrophic contributions to the momentum equation are an order of magnitude smaller and are dominated by the convection, which gives the signature of plumes aligned with the axis of rotation as shown in section 3.1.1 for the 2D case and will be discussed later on for the 3D case. This implies that for the parameter regime of Jupiter the assumption of a small Rossby number, which will be used in later analysis is valid.

Next we look at the vertical momentum balance. In the traditional shallow type system the leading order balance would be between the vertical pressure gradient and buoyancy giving hydrostatic balance (beyond the higher order basic state hydrostatic balance $\frac{\partial \bar{p}}{\partial r}=-\bar{\rho} g$ ). However due to the large aspect ratio, the Coriolis acceleration in the vertical momentum balance is not negligible. In Figure 4.2 we show that the difference between the hydrostatic terms is almost exactly the vertical momentum equation Coriolis term. This verifies that (4.3) is indeed the leading order balance. This is important when looking at thermal wind balance for the deep system which we do in the next section. Therefore we refer to the basic balance being geostrophic and hydrostatic but unlike the classic shallow fluid case it includes the non-negligible vertical Coriolis term. 


\subsection{The Vertical Structure of the Zonal Velocity}

\subsubsection{Thermal Wind for a Deep Anelastic Setting}

We begin by revisiting the thermal wind relation for a deep atmosphere. As discussed in section 4.2 unlike the traditional approximation the aspect ratio between vertical and horizontal scale is not small, and therefore the Coriolis term in the vertical equation and the one associated with vertical motion in the zonal equation are not negligible. We are interested in the effect of the Coriolis terms and the density gradient on the velocity structure. Taking the radial derivative of (4.2) and using (4.3) gives

$$
\frac{\partial u}{\partial r}=\frac{g}{2 \Omega r \bar{\rho} \sin \theta} \frac{\partial \rho^{\prime}}{\partial \theta}-\frac{1}{\bar{\rho} r} \frac{\partial \rho^{\prime}}{\partial \theta} \cot \theta u-\frac{1}{r} \cot \theta \frac{\partial u}{\partial \theta}-\frac{1}{\bar{\rho}} \frac{\partial \bar{\rho}}{\partial r} u
$$

More information would be needed to get independent expressions for the vertical and latitudinal velocity gradients, but noting that the direction parallel to the axis of rotation is given by

$$
\frac{\partial}{\partial z}=\sin \theta \frac{\partial}{\partial r}+\cos \theta \frac{1}{r} \frac{\partial}{\partial \theta}
$$

we can write the zonal velocity gradient in the direction parallel to the rotation axis as

$$
\frac{\partial u}{\partial z}=\frac{g}{2 \Omega r \bar{\rho}} \frac{\partial \rho^{\prime}}{\partial \theta}-\frac{u}{\bar{\rho} r} \cos \theta \frac{\partial \rho^{\prime}}{\partial \theta}-\frac{1}{\bar{\rho}} \frac{\partial \bar{\rho}}{\partial r} u \sin \theta
$$

This expression includes non orthogonal derivatives, unlike the standard approximation (Pedlosky, 1987) which is sufficient for a shallow system where the shear is associated with the perpendicular density gradient. In addition the zonal velocity gradient has contributions from both the vertical and latitudinal density gradients. Note that all terms on the right hand side have the mean density in the denominator. If density gradients driven by the internal convection have roughly the same scale on the top and bottom of the deep atmosphere, while the density is much bigger at the bottom rather than on top, one may expect a stronger vertical shear on top than at the bottom. We look at this more in detail in section 4.7 and show a parametrization for the shear based on scaling arguments which we compare to the numerical results. Scaling the terms in (4.6) shows that the second term on the right hand side 
is an order $\frac{\rho^{\prime}}{\bar{\rho}}$ smaller than the other terms. Then the leading order balance becomes approximately

$$
\frac{\partial u}{\partial z}=\frac{g}{2 \Omega r \bar{\rho}} \frac{\partial \rho^{\prime}}{\partial \theta}-\frac{1}{\bar{\rho}} \frac{\partial \bar{\rho}}{\partial r} u \sin \theta
$$

Therefore the shear in the direction of the rotation axis is composed of the meridional density anomaly gradient and the vertical mean density gradient. In section 4.3.2 we show numerically how each of these varies spatially.

\subsubsection{The Role of Compressibility in the Baroclinic Vorticity Production}

Another way of obtaining balance between the zonal velocity and the density gradients would be to take directly the curl of the 3D momentum equation multiplied by the full density $\rho$ giving

$$
2 \Omega \nabla \cdot(\rho \mathbf{u})-2 \Omega \cdot \nabla(\rho \mathbf{u})=\nabla \rho \times g
$$

Then, assuming the density has a mean horizontally independent hydrostatic part and a smaller anomaly (2.1), and applying the anelastic approximation (2.5) gives

$$
2 \Omega \cdot \nabla(\bar{\rho} \mathbf{u})=\nabla \rho^{\prime} \times g
$$

which is similar to (4.7). In the Boussinesq limit this gives the standard thermal wind relation. Note that if the right side would vanish this would not be the barotropic limit, since in the barotropic limit the cross product of the full density and full pressure vanishes. To see the barotropic limit we rewrite the right hand side of (4.9) as

$$
\nabla \rho^{\prime} \times g=\frac{1}{\bar{\rho}} \nabla \bar{\rho} \times \nabla p-\frac{1}{\bar{\rho}} \nabla \rho \times \nabla p
$$

where we have split both density and pressure into a hydrostatic part and a smaller anomaly $(2.1,2.2)$. In the barotropic limit the second term on the right hand side of (4.10) is identically zero, and for a geostrophically balanced fluid the first term would 
give

$$
\frac{1}{\bar{\rho}} \nabla \bar{\rho} \times \nabla p=\frac{1}{\bar{\rho}} \nabla \bar{\rho} \times(\bar{\rho} 2 \Omega \times \mathbf{u})=\mathbf{u} \nabla \bar{\rho} \cdot 2 \Omega-2 \Omega(\nabla \bar{\rho} \cdot \mathbf{u})
$$

Using the anelastic approximation and expanding the right hand side of (4.8) with (4.11) gives

$$
2 \Omega \bar{\rho} \cdot \nabla \mathbf{u}-2 \Omega \bar{\rho} \nabla \cdot \mathbf{u}=0
$$

which is the classic Taylor-Proudman theorem for a barotropic fluid (Pedlosky, 1987). Thus if the fluid is barotropic we would expect that the zonal velocity is independent of the direction parallel with the rotation axis and, if the fluid is also Boussinesq we expect that the full velocity vector is independent of this direction. We are interested though in going away from these two limits and study the role of the baroclinic effects in an anelastic fluid driven by convection. The convection would drive the density gradients away from zero, and the level of baroclinicity will set how far we are from the Taylor-Proudman theorem regime. The baroclinic form of (4.12) can be seen by taking the curl of the momentum equation (without multiplying by the density first) giving

$$
2 \Omega \bar{\rho} \cdot \nabla \mathbf{u}-2 \Omega \bar{\rho} \nabla \cdot \mathbf{u}=-\frac{1}{\bar{\rho}}\left[\nabla \bar{\rho} \times \nabla p^{\prime}+\nabla \rho^{\prime} \times \nabla \bar{p}\right] .
$$

Expressing the density in terms of pressure and entropy as in (2.20)

$$
\nabla \rho^{\prime}(p, s)=\left(\frac{\partial \rho}{\partial p}\right)_{s} \nabla p^{\prime}+\left(\frac{\partial \rho}{\partial s}\right)_{p} \nabla s^{\prime}
$$

allows rewriting the vorticity equation (4.13) for an adiabatic reference state to the highest order as

$$
2 \Omega \bar{\rho} \cdot \nabla \mathbf{u}-2 \Omega \bar{\rho} \nabla \cdot \mathbf{u}=-\frac{1}{\bar{\rho}}\left(\frac{\partial \rho}{\partial s}\right)_{p} \nabla s^{\prime} \times \nabla \bar{p}=\left(\frac{\partial \rho}{\partial s}\right)_{p} \nabla s^{\prime} \times g .
$$

Hence, equations (4.9) and (4.15) give two equivalent forms of the vorticity equation where the baroclinic terms are given once in terms of the density gradients, and once in terms of the entropy gradients. We have shown in chapter 2 that for an anelas- 

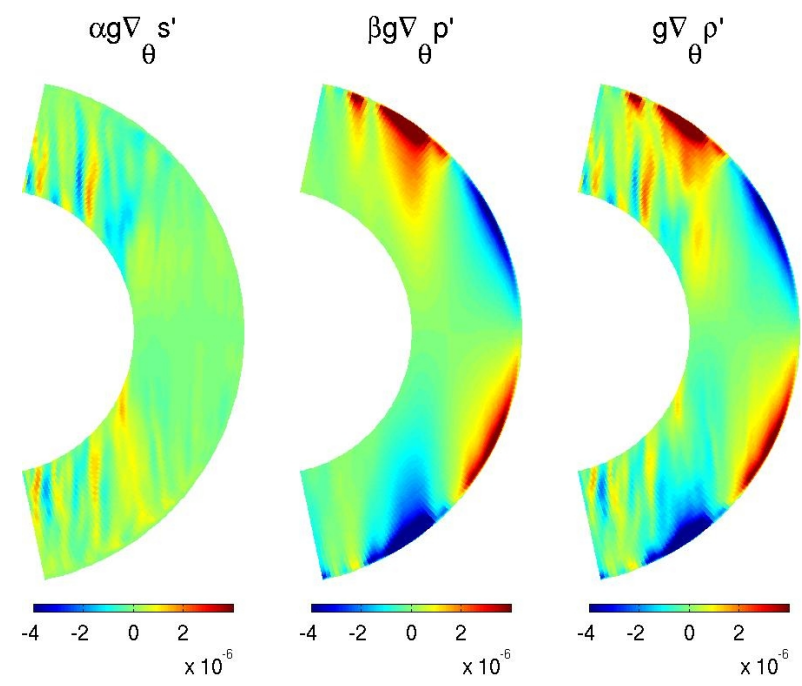

Figure 4.3: The contributions of entropy and pressure to the density anomaly and shear. left: the entropy anomaly contribution to (4.17), middle: the pressure anomaly contribution to (4.17); right: the density anomaly contribution (equal to the sum of the two left panels - equation 4.14).

tic and adiabatic fluid the buoyancy naturally is given in terms of entropy rather then density (since the background density is varying while the entropy is not). Therefore this form of the vorticity equation is consistent with the barotropic limit where the right hand side vanishes. However, while in a Boussinesq fluid the velocity divergence will vanish as well giving the standard Taylor-Proudman theorem in the anelastic case it will not and therefore the velocity gradient will depend on the compressibility.

To understand the role of the pressure gradient from (4.14) in (4.9) we consider only the zonal component of (4.9) and (4.15), so that

$$
\begin{aligned}
2 \Omega \bar{\rho} \frac{\partial u}{\partial z} & =\frac{\alpha g}{r} \frac{\partial s^{\prime}}{\partial \theta} \\
2 \Omega \frac{\partial}{\partial z}(\bar{\rho} u) & =\frac{\alpha g}{r} \frac{\partial s^{\prime}}{\partial \theta}+\frac{\beta g}{r} \frac{\partial p^{\prime}}{\partial \theta}
\end{aligned}
$$

where $\alpha$ and $\beta$ are the isentropic and isobaric coefficients in (4.14), which are defined explicitly in (2.20). Therefore subtracting (4.16) from (4.17) shows that the relation

$$
2 \Omega u \frac{\partial \bar{\rho}}{\partial z}=\frac{\beta g}{r} \frac{\partial p^{\prime}}{\partial \theta}
$$

must hold. This means that the pressure contribution to the density anomaly accounts 


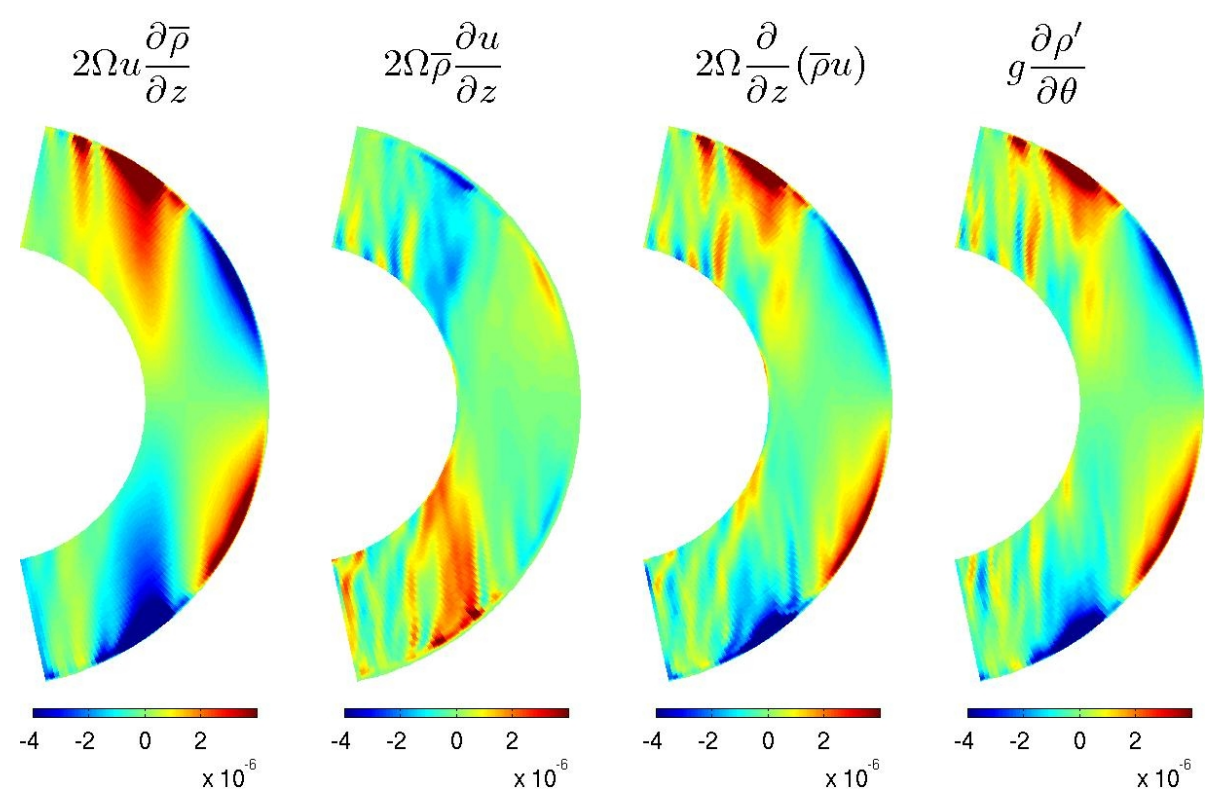

Figure 4.4: The vorticity equation balance

for the variation in the mean density. Expression (4.17) then suggests a few possible situations: if the contribution of (4.18) to the right hand side of (4.17) is small, then the zonal velocity gradient would depend on the derivative of the entropy anomalies. In the barotropic limit this would give the standard invariance of $u$ in the direction parallel to the axis of rotation, similar to the barotropic Boussinesq case. However if the contribution of (4.18) is not small then compressible effects are important and the system becomes different from the barotropic case. In a particular case where the two terms on the right hand side of (4.17) cancel each other then we expect the zonal momentum $(\bar{\rho} u)$ to be constant along the $z$ axis.

We find that in statistical steady state of our numerical simulations the system is in a state in between these two extreme scenarios and that this level of baroclinicity depends greatly on latitude. In Figure 4.3 we look at each of the terms in the vorticity equation to see its relevant contribution in (4.17). We can see that the contribution of pressure anomalies is large especially around the upper boundary while entropy contribution is larger in the interior. This is seen clearly also in Figures 3.5, 3.6 and 3.7 which are surface slices taken roughly at the top middle and bottom of the atmosphere. Near the top density anomaly is strongly influenced by the pressure anomalies while in the interior density anomalies are influenced by the entropy anomalies. If we would not have included the pressure variation contribution to density, then the density 

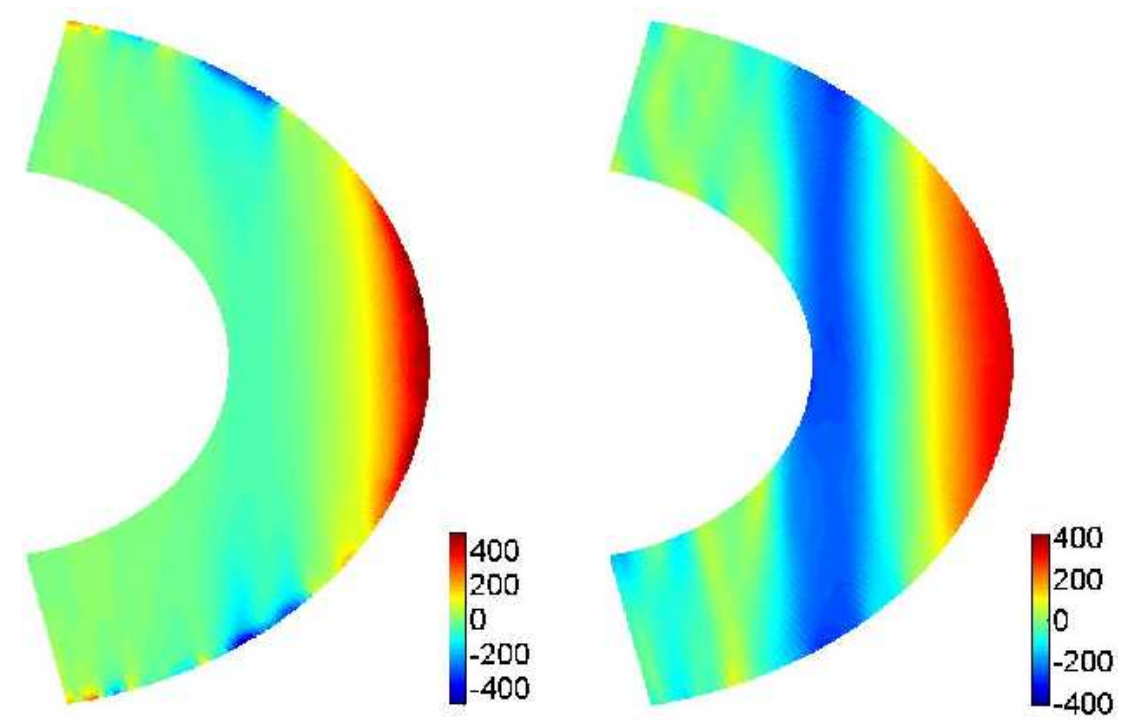

Figure 4.5: The zonally averaged zonal velocity for an Anelastic run (left) and a Boussinesq run (right). Runs differ in Rayleigh number : $R a=3 E 6$ for Anelastic and $1 E 7$ for Boussinesq where $E k=1.5 E-4$ and $\operatorname{Pr}=10$. (Figure 3.4 shows a similar plot with same Rayleigh numbers for both runs).

represented only by entropy anomalies will not be balancing the compressible part of the term on the left hand side of 4.17. In a case of small entropy anomalies this will lead to appearance of having $\bar{\rho} u$ close to constant along the direction of the rotation axis. Therefore we conclude that the pressure contribution is crucial when using the anelastic approximation. In a Boussinesq system where the system has a constant mean density the perturbation can be described by only the entropy.

In order to understand the zonal velocity vertical structure we should look at how the density contributions above contribute to the different components of equations 4.9 and 4.15. First we note that looking at the two right panels in Figure 4.4 shows that relation 4.9 holds as we expect for a small Rossby number. Then breaking this balance into its components on the two left hand side panels in Figure 4.4 shows that at low latitudes the $z$-shear of the zonal velocity itself is smaller than at high latitudes, but at the higher latitudes where the $z$-shear of zonal velocity is larger it is accompanied by a compensating shear in $\bar{\rho}$ leading to a partial cancellations of these two contributions. 


\subsubsection{Anelastic versus Boussinesq Cases}

The importance of the anelastic effects are demonstrated in Figure 4.5 where we compare two similar runs one anelastic and one Boussinesq. The anelastic case has the density varying from $0.15 \mathrm{Kg} / \mathrm{m}^{3}$ at the top level (at 1 bar of pressure) to 1983 $\mathrm{Kg} / \mathrm{m}^{3}$ at the bottom level (Figure 2.4), while the Boussinesq case is set so that the mean density is constant and equal to the weighted averaged density of the anelastic case $\left(921 \mathrm{Kg} / \mathrm{m}^{3}\right)$. In this case Anelastic and Boussinesq experiments have similar magnitudes of their zonal velocity. In Figure 3.4 we show similar 3D experiments where the anelastic and Boussinesq runs have exactly the same parameters. For the Boussinesq runs since the mean density does not depend on pressure the density anomaly is just a function of entropy and not of pressure. In Figure 4.5 we look at meridional slices comparing the zonally averaged zonal velocity fields.

Both runs have a similar velocity structure at the surface; however while the Boussinesq run is barotropic (in the $z$ direction) with strong velocities in the interior, the anelastic case has strong baroclinicity near the surface with strong shears at mid and high latitudes with a weaker baroclinic structure (though still not barotropic) closer to the equator. The meridional extent of the superrotation is similar in both cases. To look at the baroclinic structure along the $z$ axis more specifically we look at velocity sections along the $z$ axis for two runs of similar Rayleigh numbers. In Figure 4.6 each section is named by the latitude in which it outcrops at the surface.

\subsection{The Angular Momentum Balance}

In section 4.2 we showed that to the leading order in the zonally averaged zonal momentum equation the vertical and horizontal Coriolis terms would balance each other. Next we look at the dynamical balances of the zonally averaged zonal momentum equation. We divide the zonal velocity into a zonal mean and a deviation from that mean denoted by

$$
u=\bar{u}+u^{\prime}
$$

Then to the leading order

$$
\frac{\partial \bar{u}}{\partial t}+\frac{\overline{u w}}{r}-\frac{\overline{u v}}{r} \tan \theta-2 \Omega \sin \theta \bar{v}+2 \Omega \cos \theta \bar{w}+\frac{1}{\bar{\rho}} \nabla \cdot(\bar{u} \bar{\rho} \overline{\mathbf{u}})+\frac{1}{\bar{\rho}} \nabla \cdot\left(\overline{\bar{\rho} u^{\prime} \mathbf{u}^{\prime}}\right)=\nu \nabla^{2} \bar{u}
$$



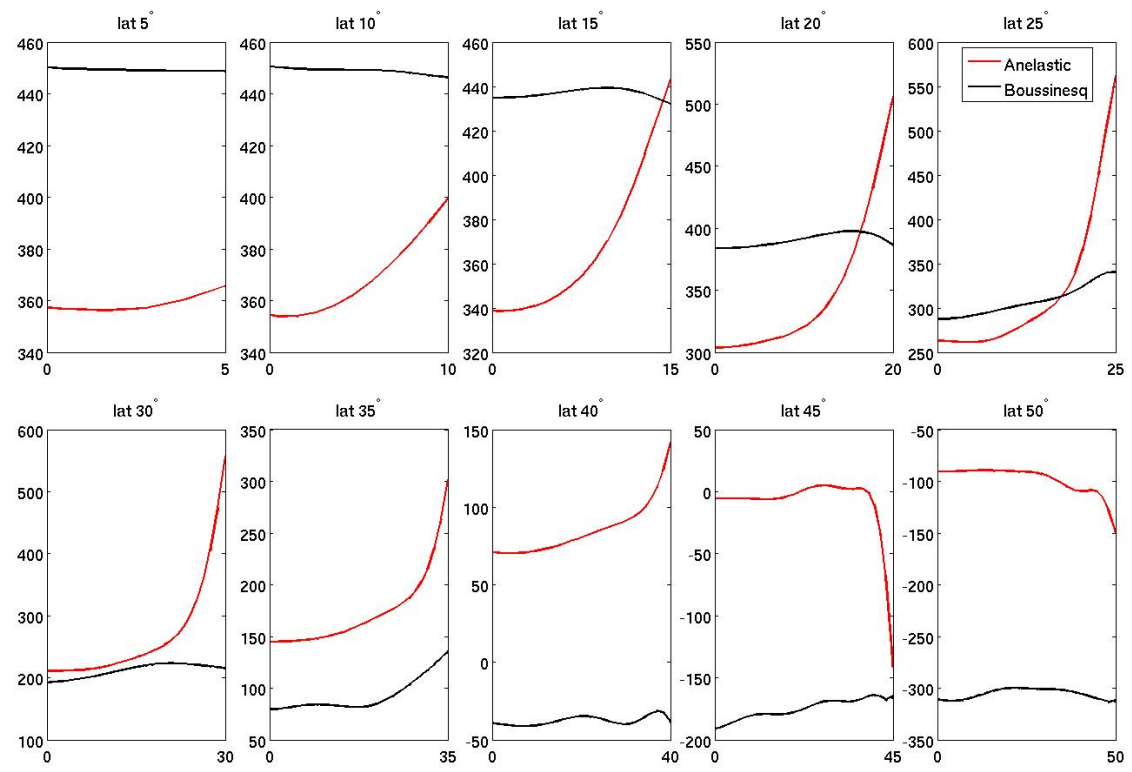

Figure 4.6: Zonally averaged zonal velocity for Anelastic and Boussinesq runs along slices parallel to the axis of rotation. Each slice goes from the surface (denoted by the latitude) to the equatorial plane.

where zonal averaging is denoted with the bar. Beyond the lowest order geostrophic balance between the Coriolis terms in (4.20) as implied by (4.1), we find looking at the numeric values that to the next order the leading terms are the eddy momentum flux divergence and the viscous flux so that

$$
-2 \Omega \sin \theta \overline{v_{a}}+2 \Omega \cos \theta \overline{w_{a}}+\frac{1}{\bar{\rho}} \nabla \cdot\left(\overline{\rho u^{\prime} \mathbf{u}^{\prime}}\right) \approx \nu \nabla^{2} \bar{u}
$$

where we denote with the subscript the next order component. Since the variations along the axis of rotation are small, then when looking on the equatorial plane (the equatorial line in the zonally averaged picture) the leading order balance is

$$
\frac{1}{\rho} \frac{\partial}{\partial r}\left(\overline{\bar{\rho} w^{\prime} u^{\prime}}\right) \approx \frac{\nu}{r^{2}} \frac{\partial^{2}\left(r^{2} \bar{u}\right)}{\partial r^{2}}
$$

where in fact only the higher order viscosity derivatives are significant. In Figure 4.7 we show both components of the momentum flux divergence for a section along the equator. It shows that the momentum flux divergence is dominated by the radial fluxes. The momentum fluxes are outward and big in a localized region. This mo- 


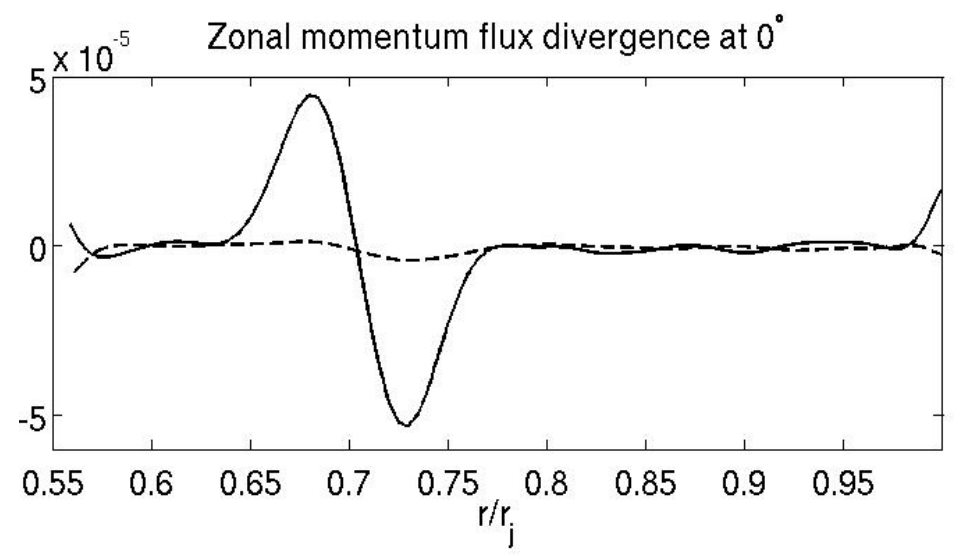

Figure 4.7: The radial and latitudinal (dashed) contributions to the zonal momentum flux divergence as function of radius at the equator.

mentum transfer is the basis for understanding the circulation of the model and the formation of the equatorial superrotation. We discuss this further in section 5.1.

It is convenient to rewrite equation (4.20) in terms of the angular momentum

$$
M=\Omega r^{2} \cos ^{2} \theta+u r \cos \theta
$$

so that

$$
\frac{\partial \bar{M}}{\partial t}+\frac{1}{\bar{\rho}} \nabla \cdot(\bar{\rho} \overline{\mathbf{u}} \bar{M})+\frac{1}{\bar{\rho}} \nabla \cdot\left(\bar{\rho} \overline{\mathbf{u}^{\prime} M^{\prime}}\right)=\nu \nabla^{2} \bar{M}
$$

where we have split the angular momentum into a perturbation and a zonal mean. Integrating this equation multiplied by the mean density over a volume contained by the exterior surface and a constant angular momentum surface (which is nearly parallel to the axis of rotation because of the dominance of the first term in $M$ ), will cause the contribution from the mean fluxes to vanish since

$$
\int \nabla \cdot(\bar{\rho} \mathbf{u} \bar{M}) d V=\bar{M} \int \nabla \cdot(\bar{\rho} \mathbf{u}) d V=0
$$

Therefore in steady state friction is necessary to balance the angular momentum eddy fluxes. This also shows that, for the 2D case, no mean zonal circulation can form. For the 3D case only eddy angular momentum fluxes can carry angular momentum cross mean angular momentum contours (although locally mean fluxes can do so as well). Considering the meridional plane streamfunction shown in Figure 3.4 the fact 

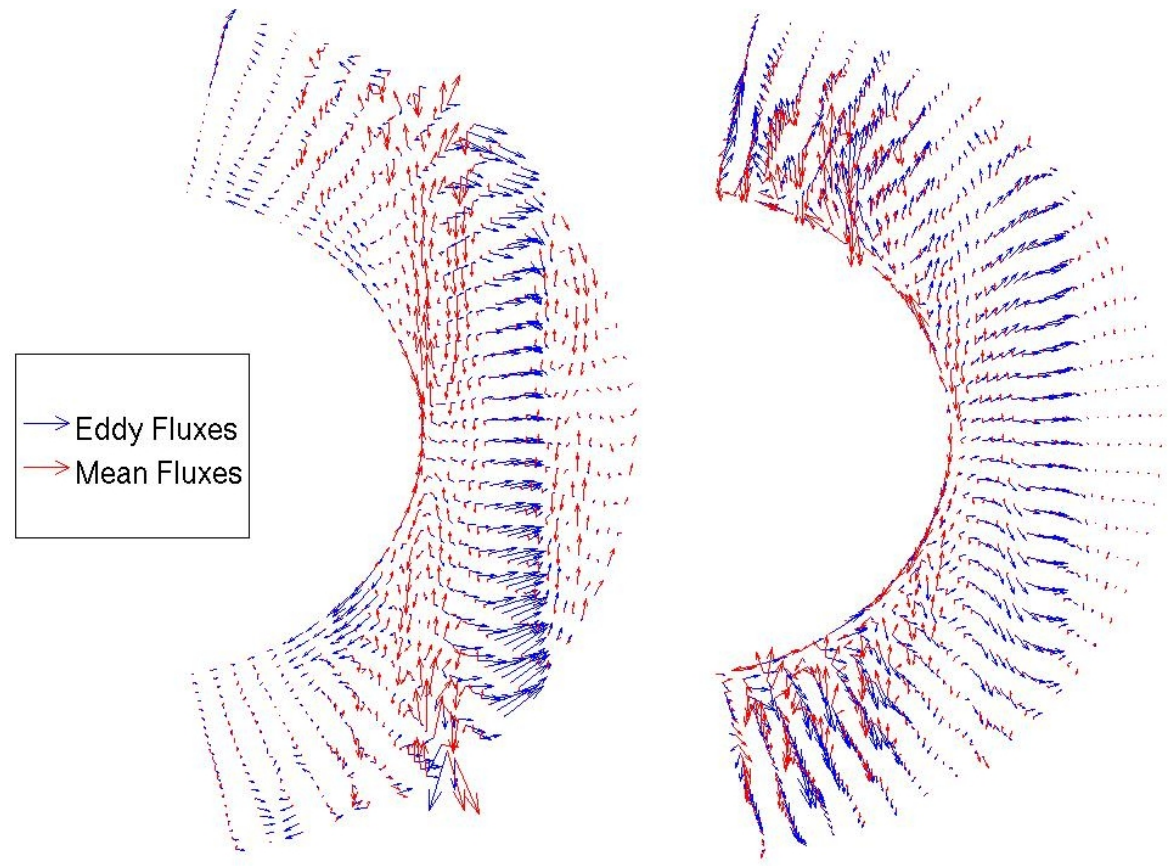

Figure 4.8: Angular momentum (left) and heat (right) mean (red) and eddy (blue) fluxes in a meridional cross section.

that the zonally averaged (or 2D) meridional circulation is confined to narrow bands along the axis of rotation is related to the fact that the mean circulation can not cross angular momentum contours which are parallel to the axis of rotation. The width of these bands will be related therefore to the magnitude of the viscosity, and we expect that in the limit of small Ekman number these convective mean meridional circulation bands will become narrower. Comparing the angular momentum mean fluxes (without the solid body component of $M$ ), to the angular momentum eddy fluxes in Figure 4.8, we find that while the mean fluxes transfer angular momentum mainly parallel to the mean angular momentum contours, the eddy fluxes transport the angular momentum across mean angular momentum contours to low latitudes. This mechanism is most prominent in the region outside the tangent cylinder where the large scale columnar structures interact with the mean shear. This transfer of angular momentum through the turbulent fluxes to the equatorial outer regions of the planet drives the equatorial surface superrotation. We discuss this mechanism in chapter 5 .

The right hand panel shows the equivalent eddy heat and mean heat fluxes. As opposed to the angular momentum, there are strong heat fluxes also in high latitudes. 


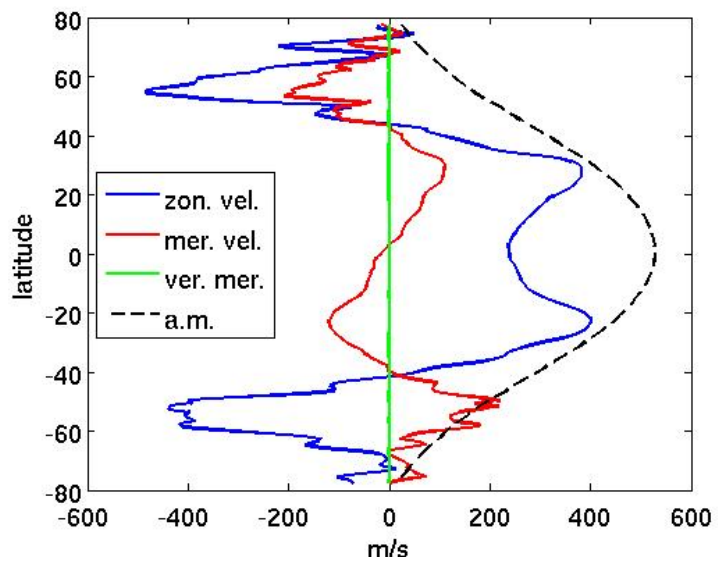

Figure 4.9: The zonal (blue), meridional (red) and vertical (green) zonally averaged surface velocities for a run with parameters: $R a=1 E 7, E k=1.5 E-4$ and $\operatorname{Pr}=10$. The dashed line is the normalized mean angular momentum.

This transfer of heat mainly parallel to the rotation axis moves heat from lower to higher latitudes (a section parallel to the rotation axis outcrops in a higher latitude in the upper boundary than in the lower boundary). This results in heating of the polar regions. We hypothesize that this mechanism of heat transport to higher latitudes by internal mean heat fluxes parallel to the axis of rotation can balance the solar heating resulting in the observed flat emission on Jupiter and Saturn. Figure 4.9 shows the zonally averaged surface velocities and normalized mean angular momentum. At low latitudes we find a Hadley cell (weaker than the zonal flow) which is driven by the equatorial upwelling seen in Figure 4.8. Exterior to the tangent cylinder containing the eddy angular momentum flux convergence we find an inverse meridional cell (surface flow away from the pole), which is a surface return flow driven by the poleward heat flux. The latitude where eddy angular momentum fluxes are zero, meaning that the mean surface zonal velocity is zero, is also where the meridional surface flow vanishes due to the relation between the meridional velocity and the eddy flux divergence (4.21).

\subsection{The Effect of Rotation}

We have seen that for the parameter regime of Jupiter and Saturn Rossby numbers are small and therefore rotation is important in the basic balances. In the 2D Boussinesq 


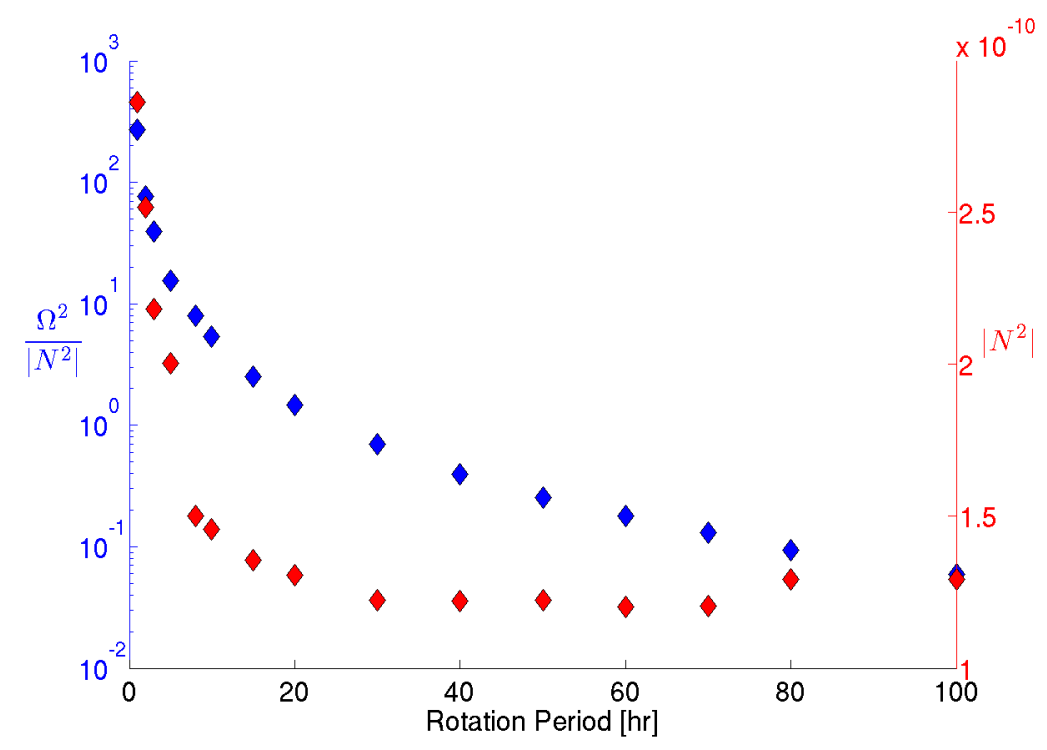

Figure 4.10: The ratio $\frac{\Omega^{2}}{\left|N^{2}\right|}$ and the value of $\left|N^{2}\right|$ as a function of the rotation period.

runs we have shown that the ratio of $\frac{\Omega^{2}}{N^{2}}$ is an important measure for characterizing the flow. For the anelastic case due to having a mean state with a density gradient the buoyancy frequency is defined in terms of entropy. We show this by differentiating the linear non-rotating case of equation (2.26) in time, which gives

$$
\frac{\partial^{2} w}{\partial t^{2}}-w \frac{\partial s^{\prime}}{\partial r} \frac{\partial \bar{T}}{\partial r}=-\frac{\partial^{2} \Phi}{\partial r \partial t}
$$

where we have used relation (2.28) as well. Therefore for the anelastic system the equivalent to the traditional Brunt-Vaisala frequency is

$$
N^{2}=-\frac{\partial s^{\prime}}{\partial r} \frac{\partial \bar{T}}{\partial r}
$$

Since in (2.26) the temperature gradient replaced gravity, and we have shown that entropy rather then density is the natural variable for buoyancy in the anelastic system, then this buoyancy frequency is the natural outcome. For the convective system however this value becomes negative. In the $2 \mathrm{D}$ system convection was concentrated in specific regions and therefore for most cases the mean $N^{2}$ when averaged over the whole domain was still positive, however for the 3D experiments shown here the mean $N^{2}$ is negative. Still, the absolute value (although not a buoyancy frequency) gives 


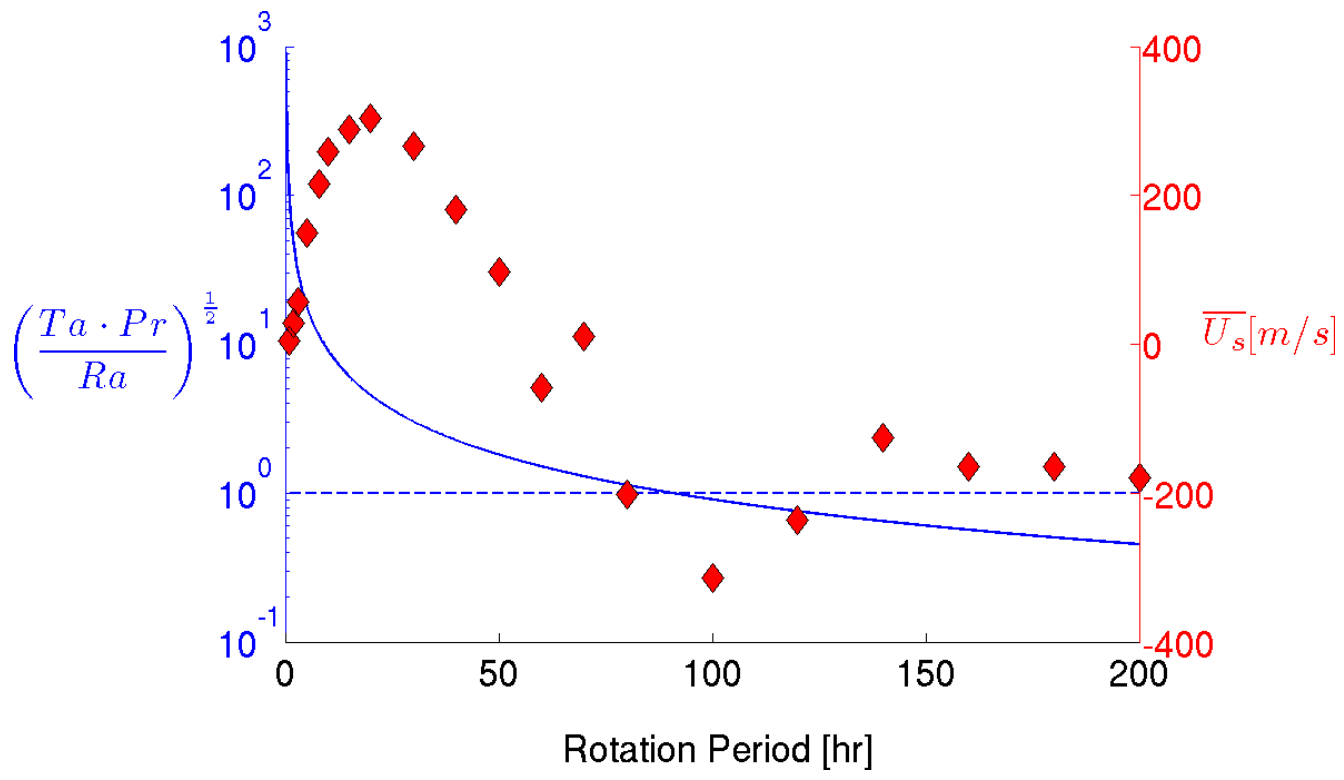

Figure 4.11: The nondimensional number $\chi=\left(\frac{T a \cdot P r}{R a}\right)^{\frac{1}{2}}$ as function of the rotation period and the mean zonal velocity at the surface averaged around the equator as function of the rotation period for a set of experiments with equal parameters but varying rotation period $R a=3 E 6, \operatorname{Pr}=10$.

a good measure for the intensity of the convection. In Figure 4.10 we show a set of experiments where we vary the rotation period for a given model configuration. We find that the value of $\left|N^{2}\right|$ grows (even though the Rayleigh number is kept constant) with faster rotation period, but $\frac{\Omega^{2}}{\left|N^{2}\right|}$ decreases as the rotation period grows, and to a reasonable approximation when $\frac{\Omega^{2}}{\left|N^{2}\right|}$ is less than one the flow is no longer aligned with the rotation axis. When $\frac{\Omega^{2}}{\left|N^{2}\right|}>1$ the flow is aligned with the rotation axis.

As discussed in section 3.1.1 a similar measure which is better defined in terms of convection and uses the nondimensional parameters of our system is

$$
\chi=\frac{T a \cdot \operatorname{Pr}}{R a}
$$

In Figure 4.11 we plot this parameter as function of the rotation period. For the set of parameters of this experiments at a rotation period of 85 hours $\chi=1$. As seen in previous sections for the rotation period of Jupiter and Saturn the velocities are aligned with the rotation axis characterized by strong superrotation around the equator. On the same plot we show also the mean surface zonal velocity around the equator for these runs. We find that at about 50 hours the velocity changes from being 
positive (eastward velocity) to negative. The numerical experiments with fast rotation period all have very similar velocity profiles, characterized by strong superrotation at the equator. Beyond a rotation period of 50 hours though, the nature of the dynamics changes quite rapidly and the zonal mean develops large closed circulations in the $\mathrm{r}-\theta$ plane with no alignment with the rotation axis. This is similar to what we have shown for the $2 \mathrm{D}$ case (Figure 3.2), however this circulation is also accompanied with subrotation at the equator. In Figure 4.12 we show the zonally averaged velocity for two examples out of this set of runs, one with the rotation period of Jupiter (9.92 hours), and the second with a rotation period of 80 hours. We find one of these two states to appear for the whole range of experiments presented in Figures 4.10 and 4.11. The transition between the two states at a rotation period of 50 hours is very rapid. The estimate for this transition based on $\frac{\Omega^{2}}{N^{2}}$ is at 30 hours, but since by averaging $N^{2}$ we are approximating the mean buoyancy in the whole domain this is estimate seems within the reasonable error. The estimate based on the limit $\chi=1$ is at 85 hours.

\subsection{Properties in the Zonally Asymmetric Circula- tion}

So far we have looked at the zonally averaged fields in the 3D model. The differences between the $2 \mathrm{D}$ and the $3 \mathrm{D}$ flow indicate that zonal asymmetries are important for the $3 \mathrm{D}$ circulation. We have seen that eddy momentum fluxes carry momentum away from the axis of rotation to the outer equatorial part. Next we look at the zonal structure of the circulation.

\subsubsection{Formation of Columnar Convection}

Looking at the equatorial plane the most prominent feature beyond the strong prograde velocities near the upper boundary and the retrograde velocities near the inner boundary are large positively rotating (in respect to the rotation of the planet) eddies in the interior. Busse (1976) has suggested that Taylor columns can form around a hot convective interior and the interaction of the columns can drive the jets in the atmosphere. Zhang and Schubert (1996) have shown formation of convection cells in a Boussinesq 3D model for Rayleigh-Benard type convection. Here we use the anelastic 


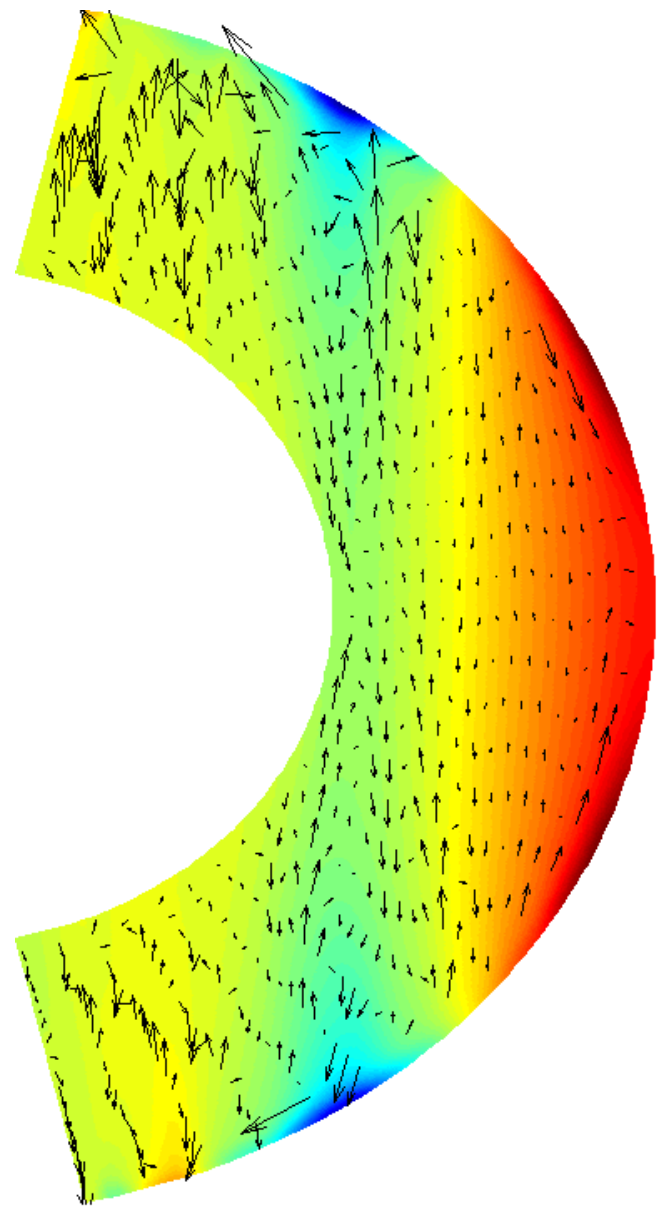

$-500$

500
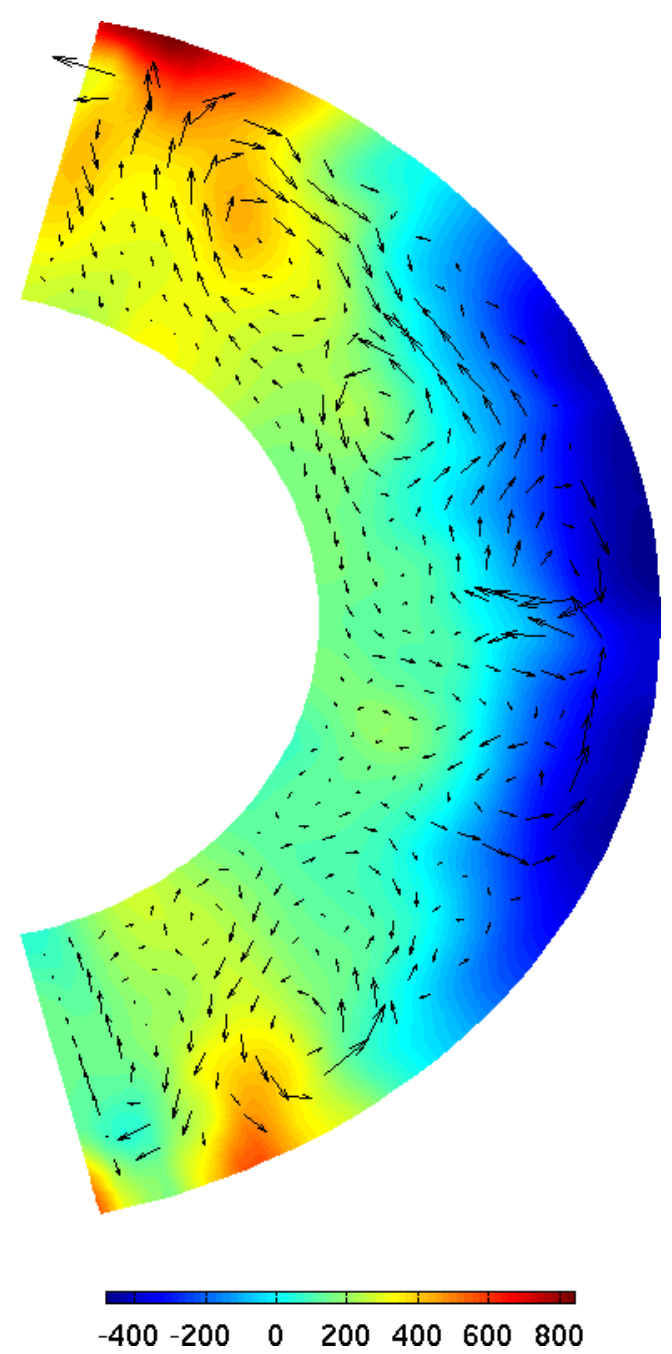

Figure 4.12: The effect of rotation: Velocity fields for a fast and slow rotating planet. (left) rotation period of 9.92 hours; (right) rotation period of 80 hours; In color are the zonal mean zonal velocities where red is eastward, and the arrows are the zonally averaged radial and meridional velocities. 

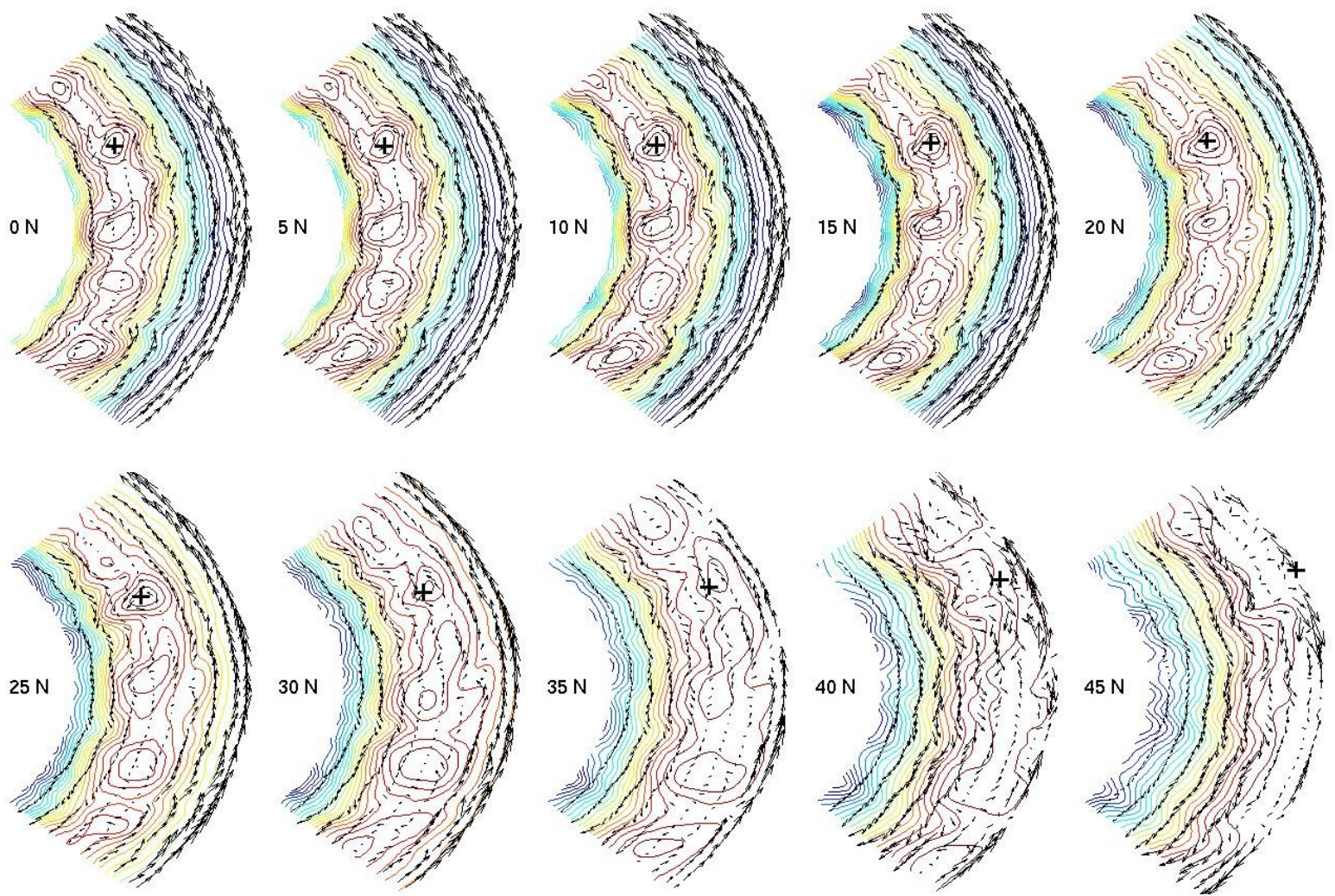

Figure 4.13: The 2D streamfunction on slices oriented toward the center of the planet (radius-longitude surfaces), showing the formation of Columns which are driven by the convection. Plus signs are located at an equal distance from the rotation axis in all panels, and located within one of the columns, showing that these columns are parallel to the axis of rotation.

model to show the formation of such columnar structures that extend almost from one boundary to the other crossing the equatorial plane at about $2 / 3$ the planetary radius. In Figure 4.13 we show the 2D streamfunction on slices along the longituderadius planes on constant latitude surfaces (so that the surfaces are not parallel). The slices are spread apart in $5^{\circ}$ in latitude going northward. The closed structures on the equatorial plane (upper left panel) extend out in radius as they move out in latitude so that they are parallel to the rotation axis. To demonstrate this we have marked the center of one of the columns on the equatorial plane with a plus sign, and the plus signs on the other planes have an equal distance to the rotation axis, and the same longitudinal angle. We find these columnar features to be a robust feature in all numerical experiments. 


\subsection{Scaling Estimates for the Vertical Profile of the Zonal Wind}

We try to estimate the scale of the density gradients driven by the convection. This is beneficial for understanding whether the representation of convection in our model can be interpreted in terms of simple scaling arguments; also by estimating the density gradients, we hope to have an estimate for the zonal velocity vertical shear. It is important to distinguish between the density gradients from the convective plumes, and the larger scale geostrophically balanced density gradients. We begin by estimating the amplitude of the velocities driven by the convection and comparing them to the corresponding velocities obtained by our numerical model. Following Fernando

et al. (1991), and Ingersoll and Pollard (1982) we estimate the mean heat flux carried by convection as

$$
F=\bar{\rho} C_{p} w^{\prime} \Delta T,
$$

where $\bar{\rho}$ is the mean density, $C_{p}$ is the specific heat (which we can calculate from the EOS properties (Kippenhahn and Weigert, 1990), and is a function of depth), $\Delta T$ is the temperature across the plumes and $w^{\prime}$ is the convectively driven vertical velocity. Due to the rotation we can relate the production of vorticity and the buoyancy anomaly via the vertical momentum balance which gives a balance between the Coriolis force and the buoyancy so that

$$
\Omega u^{\prime}=\frac{\alpha g \Delta T}{\bar{\rho}} .
$$

Now we can write an expression for the correlation of these two velocities as a function of the thermodynamic variables and the heat flux so that

$$
u^{\prime} w^{\prime}=\frac{\alpha g F}{\bar{\rho} C_{p} \Omega} .
$$

All variables on the right hand side of (4.27) are given by the EOS and the reference state of the model. The flux can be inferred from the prescribed radial heating profile. The forcing as applied to the model assumes the vertical profile is close to adiabatic and that the planet is cooling on very long time scales. The forcing is applied to the 


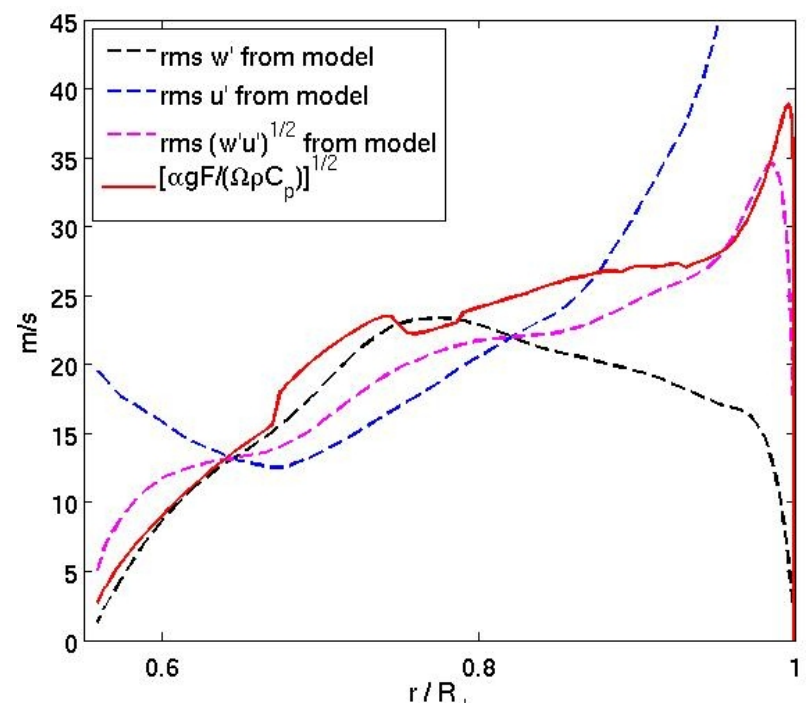

Figure 4.14: $u^{\prime} w^{\prime}$ estimated from scaling arguments and the rms from the model. The plot has the model output for $u^{\prime}$ and $w^{\prime}$ separately where $w^{\prime}$ is bounded to zero at the upper boundary while $u^{\prime}$ has a slip condition, and the combined $\left(u^{\prime} w^{\prime}\right)^{\frac{1}{2}}$.

heat budget as a heat source $Q$ given by

$$
\frac{\partial s^{\prime}}{\partial t}+\frac{1}{\bar{\rho}} \frac{\partial}{\partial r}\left(\bar{\rho} \overline{s^{\prime} w^{\prime}}\right)=\frac{Q}{\bar{T}}=\nabla \cdot F
$$

which when integrated over the volume is zero. Therefore we can calculate the effective flux $(F)$ at each depth from the heating by

$$
F=\frac{1}{r^{2}} \int \frac{\bar{\rho} Q}{\bar{T}} r^{2} d r+F_{0}
$$

where $F_{0}$ is zero since the flux at the bottom is zero. Comparing the right hand side term in (4.27) shows a good agreement with the eddy rms velocities given by the model, this is shown in Figure 4.14. This means that our convectively driven velocities are on average well approximated by these arguments, even though the convective velocities themselves are stronger than what we expect on Jupiter because the heat flux prescribed to the model is stronger than the heat flux we expect to find on Jupiter.

A common feature of numerical models is that the forcing (in terms of heat flux) must exceed in orders of magnitude what we believe exists in the interiors of the giant planets (which is on the order of $10 \frac{\mathrm{W}}{\mathrm{m}^{2}}$ on Jupiter (Hanel et al., 1981), and 
even less on Saturn (Hanel et al., 1983)). The reason for this over-forcing is that due to numerical grid size limitations the turbulent viscosities and diffusivities used in numerical models averages the turbulence in a grid box rather than represent the molecular value, and therefore the Ekman numbers are orders of magnitude too large. This means that to reach flux Rayleigh numbers which exceed critical and are as turbulent as numerics allows, the large viscosities and diffusivities must be compensated by effectively large fluxes exceeding the values we believe exist on the giant planets. In fact, even when over-prescribing the fluxes, the Rayleigh numbers are many order of magnitude smaller the expected planetary ones. Therefore these numerical models should be thought of only in terms of the nondimensional parameters and not in terms of the actual heat fluxes, viscosities, diffusivities etc. Nevertheless, our objective is to infer from these models actual characteristics of the planet and overforcing the heat flux is a problem we should address. Therefore we present our numerical results for a range of Rayleigh numbers in order to show the dependence on the forcing, still being away from real planet values which will require molecular size grid not achievable with current computational abilities.

The result in Figure 4.14 shows that even though we are overforcing the system the scaling arguments still hold, resulting in higher turbulent velocities than we believe exist in the interior of the planet. However since the model mean velocities (not convective) are of the right order of magnitude and for small Rossby numbers are geostrophically balanced, the mean densities are well represented. Bridging this gap between the overforcing and the resulting scales is a major challenge of numerical modeling in convective systems.

Away from the boundaries we see in Figure 4.14 that the rms zonal and vertical anomaly velocities are of the same order. Due to the slip boundary condition they differ along the boundaries. Therefore for the interior if we assume that $u^{\prime} \sim w^{\prime}$, we can get an estimate for the convective density gradients by using (4.26) and (4.27) so that

$$
\Delta \rho^{\prime}=\left(\frac{F \alpha \Omega \bar{\rho}}{g C_{p}}\right)^{\frac{1}{2}} .
$$

This gives an estimate to the turbulent density anomalies, and therefore an upper limit to the steady state geostrophically balanced density gradients. Relating the convective density anomalies to the mean geostrophic ones is the main leap of this 


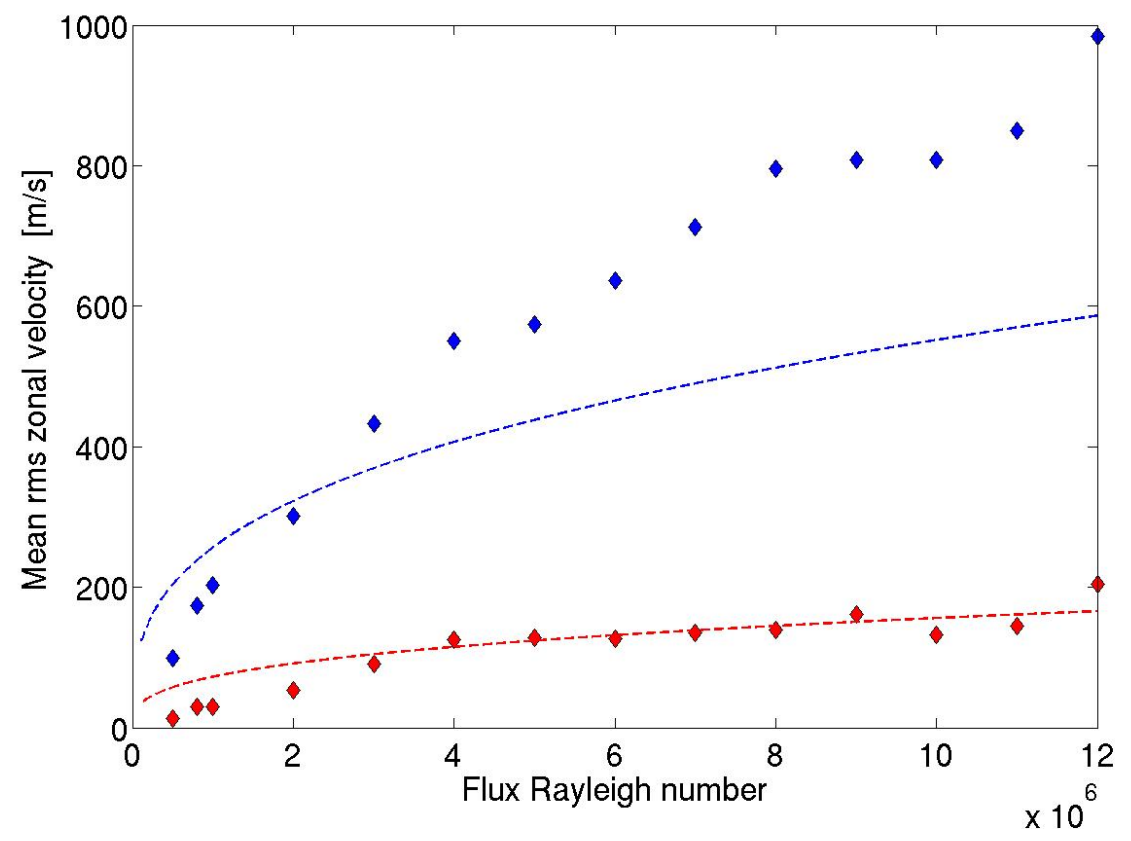

Figure 4.15: Dashed lines are the approximation for typical zonal velocities from (4.33) for 4 bar (blue) and 10 Mbar (red) as a function of Rayleigh number. Dots are the corresponding mean rms zonal velocity values from the numerical model.

approximation and therefore we treat this as an upper limit. Now we use this scale of the mean density gradients to estimate the geostrophic velocities and shears.

In the numerical results presented in the previous section we showed that for Jupiter and Saturn type parameters the Rossby number is small, and there are two different length scales in the problem. One scale is the planetary scale and we take this to be the scale of the planet denoted by $R$. The second scale is the scale of the large columnar cells (driven by convection but are larger than the convective length scales), which we denote as $L$. The vorticity of these columns can be produced in two ways: one is the by stretching the columns and then the rate of vorticity generation is given by

$$
\frac{u}{L \tau}=\frac{2 \Omega u}{R}
$$

where $u$ is the scale of the mean velocity, and $\tau$ is a time scale. The second way of 
producing vorticity is by the curl of the buoyancy force (4.9) which can be scaled as

$$
\frac{u}{L \tau}=\frac{g \Delta \rho}{\bar{\rho} L}<\frac{1}{L}\left(\frac{g \alpha F \Omega}{\bar{\rho} C_{p}}\right)^{\frac{1}{2}}
$$

where we have used the upper limit for the density gradients as given by (4.30). We assume that for the large scale motions the relevant time scale is the advective time scale $\tau=\frac{L}{u}$ (alternatively one can assume the time scale is $\frac{1}{\Omega}$, this would give back an equivalent to (4.27) as an upper limit, because assuming the upper limit in (4.32)). Plugging the advective time scale in the equations for production of vorticity $(4.31,4.32)$ gives a scale for the mean zonal velocity as function of the thermodynamic properties and the forcing

$$
u=\left(\frac{R g \alpha F}{\bar{\rho} C_{p}}\right)^{\frac{1}{3}} .
$$

The values given by this expression give a good order of magnitude estimate to the velocities given by the model. The question is can we infer from this, the velocities on the real planet with planetary type fluxes? First we note that comparing (4.33) for the atmosphere at 1 bar to the interior at $1 \mathrm{Mbar}$ the density increases by 4 orders of magnitude and the thermal expansivity will decrease by 3 orders so we can expect the interior velocities to be substantially smaller than the atmosphere ones. In Figure 4.15 we compare the rms velocities at 4 bar and 10 Mbar in our model to the velocities inferred from (4.33) for different Rayleigh numbers. We keep the viscosity constant so the change in the Rayleigh numbers reflects the change in flux. The scaling seems to be robust for a range of Rayleigh numbers for the interior values, while for the atmosphere (though still giving right orders of magnitude) the scaling gives less than model values (a problem for the atmosphere scaling is that the effective forcing for the uppermost level is zero (4.29) so we must look at a few levels below and therefore we look at the 4 bar level and not the 1 bar level which is the upper most level of the model) . Applying Jupiter values of $F=10 \frac{\mathrm{W}}{\mathrm{m}^{2}}, \alpha=10^{-2} \frac{1}{K^{\circ}}, R=7 E 7 \mathrm{~m}$, $C_{p}=1.3 \cdot 10^{4} \frac{J}{K g K^{\circ}}$, and $\rho=0.1 \frac{K g}{m^{3}}$ for the atmosphere, and $F=10 \frac{W}{m^{2}}, \alpha=10^{-5} \frac{1}{K^{\circ}}$, $\rho=10^{3} \frac{\mathrm{Kg}}{\mathrm{m}^{3}}$ for the interior, we find velocities on the orders of $50 \mathrm{~m} / \mathrm{s}$ at the 1 bar level and $0.03 \mathrm{~m} / \mathrm{s}$ for the interior. This shows a significant change in zonal velocities between the atmosphere and the interior. To further examine the vertical profile and to address the issue of the over forcing, we look at zonal velocity profiles along sections 

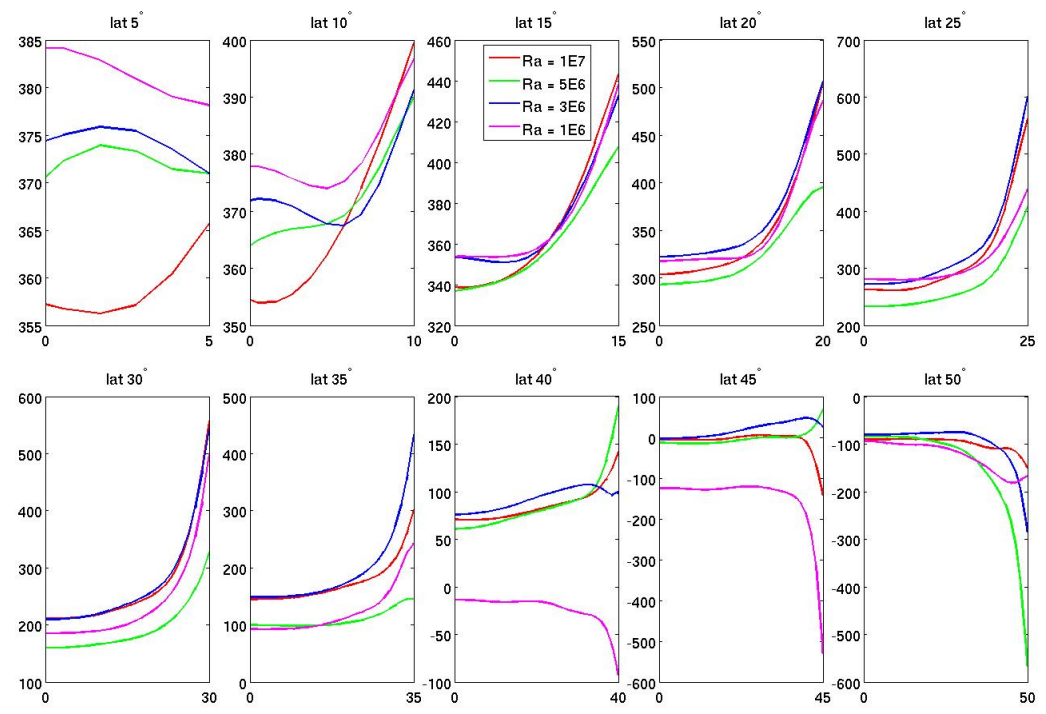

Figure 4.16: Zonally averaged zonal velocity $\left(\mathrm{ms}^{-1}\right)$ along slices parallel to the axis of rotation. Each slice goes from the surface (denoted by the latitude) to the equatorial plane. Similar velocity profiles are shown for four experiments with different Rayleigh numbers of $1 E 7,5 E 6,3 E 6$ and $1 E 6$, other parameters in these runs are $E k=1.5 E-4$ and $\operatorname{Pr}=10$. The velocity is scaled by Rayleigh number to show the similar profiles. The velocity values matches that of $R a=1 E 7$. Scaling to the velocity can be inferred by Figure 4.15 .

parallel to the rotation axis (denoted by the latitudes at which the sections cross the top surface) in Figure 4.16. The sections are separated in 5 degrees in latitude. These sections show a baroclinic structure of the velocity which has a latitudinal dependence due mainly to the variation in density and thermal expansivity which have different profiles along different sections. An important point regarding the overforcing is that the profiles (which are normalized by the Rayleigh number ratio) do not depend on Rayleigh number. This means that although the value of the velocity depends on Rayleigh number the baroclinic profile does not, and therefore the result of weaker zonal velocities in the interior is robust, and it roughly matches the scaling given by (4.33). Note that for a constant forcing and thermal expansivity, the vertical profile of velocity will go inversely with $\rho^{\frac{1}{3}}$, a state in between the barotropic limit and momentum column limit presented in section 4.3.1. All this suggests that with a strong vertical variation in density, the velocity can vary substantially from the atmosphere down to the interior. This is demonstrated well when comparing the anelastic to Boussinesq models in Figure 4.5. 


\section{Chapter 5}

\section{Weakly Nonlinear Analysis of Column Formation and Superrotation}

In this chapter we focus on the mechanisms leading to the dynamics seen in the fully turbulent model presented in chapter 3 . We have shown formation of equatorial superrotation, rotating cyclonic columns parallel to the rotation axis and a strong shear in the vertical structure of the wind. We have seen that upgradient angular momentum eddy fluxes drive angular momentum perpendicular to the axis of rotation and contribute to the superrotation. However, we have not answered the question of why are the fluxes pointed in that direction? why do we find only cyclonic convection columns? why do the columns propagate? and what sets the number of columns around the sphere? In this chapter we answer these questions.

In this analysis we use the full GCM, a simplified analytical model and a simplified single layer type numerical model. We look at the GCM in a parameter regime where convection is weak, and allows us to examine the dynamics while nonlinear effects are small. We can then understand the preference for positive shear and prograde rotation and show the transition from a state with weak cyclones and anticyclones on the equatorial plane to one dominated by only cyclones. Then in section 5.2 we look at a simplified model of a single column (Ingersoll and Pollard, 1982) parallel to the axis of rotation and show how a Rossby wave type mechanism explains the direction of propagation and the number of columns. In section 5.3 we present another simplified model of a shallow water annulus and show how this model demonstrates some of the dynamics seen in the full GCM. 


\subsection{The Weakly Nonlinear Limit}

We begin with looking at the 3D model results in the limit of small Rayleigh and Prandtl numbers. We find that in this limit the solution initially looks like linear solutions to the problem of convection in a rotating sphere as shown by Zhang (1992) and Zhang and Schubert (1997), and then goes to a state which is qualitatively similar to the one we see in the fully turbulent experiment shown in chapter 3 . In the new state the flow has only columnar cyclones rotating around the equatorial plane. This weakly nonlinear solution allows us to understand the physical mechanism seen in the fully turbulent cases. Figure 5.1 shows snapshots of the equatorial streamfunction (see definition in section 3.3) as it evolves in time beginning from spin-up, and reveals two very distinct regimes.

The first regime, while the velocities are small (we begin with zero velocity), is a series of equally spaced cyclonic and anticyclonic vortices on the equatorial plane. They propagate eastward and spiral radially (see Figure 5.1). In section 5.2 we discuss the Rossby wave type mechanism causing the eastward propagation. The spiraling of the phase lines is due to a larger planetary vorticity gradient in the outer region. We discuss and demonstrate this in section 5.3. Initially since the velocities are small the nonlinear contributions to the dynamics are weak, providing an equivalent linear solution. Several authors (Zhang and Busse, 1987; Busse, 1994; Zhang and Schubert, 1997) have looked at the linear problem of convection in a spherical rotating shell. Zhang and Schubert (1997) solve the linear problem for a Boussinesq fluid where the flow is driven by an internal heating profile. The solutions they find for the velocities and the temperature fields are given as an analytic expression in terms of spherical harmonic Legendre polynomials and spherical Bessel functions. These solutions look very similar to our solution in this first regime. Therefore as long as the perturbation is small and the flow is close to linear our solutions match previous linear analysis.

The system is constantly driven by the convection and therefore in time (while the effect of dissipation is small), the velocities become larger. As they become stronger due to the tilt in the direction of the convection columns as given by the linear solution (there is a correlation between the direction of zonal and radial velocities) angular momentum is fluxed to the outer parts of the sphere creating a vertical shear. As the shear becomes stronger, with eastward zonal velocity towards the outer boundary and westward flow towards the inner boundary, the anticyclones can not survive against 

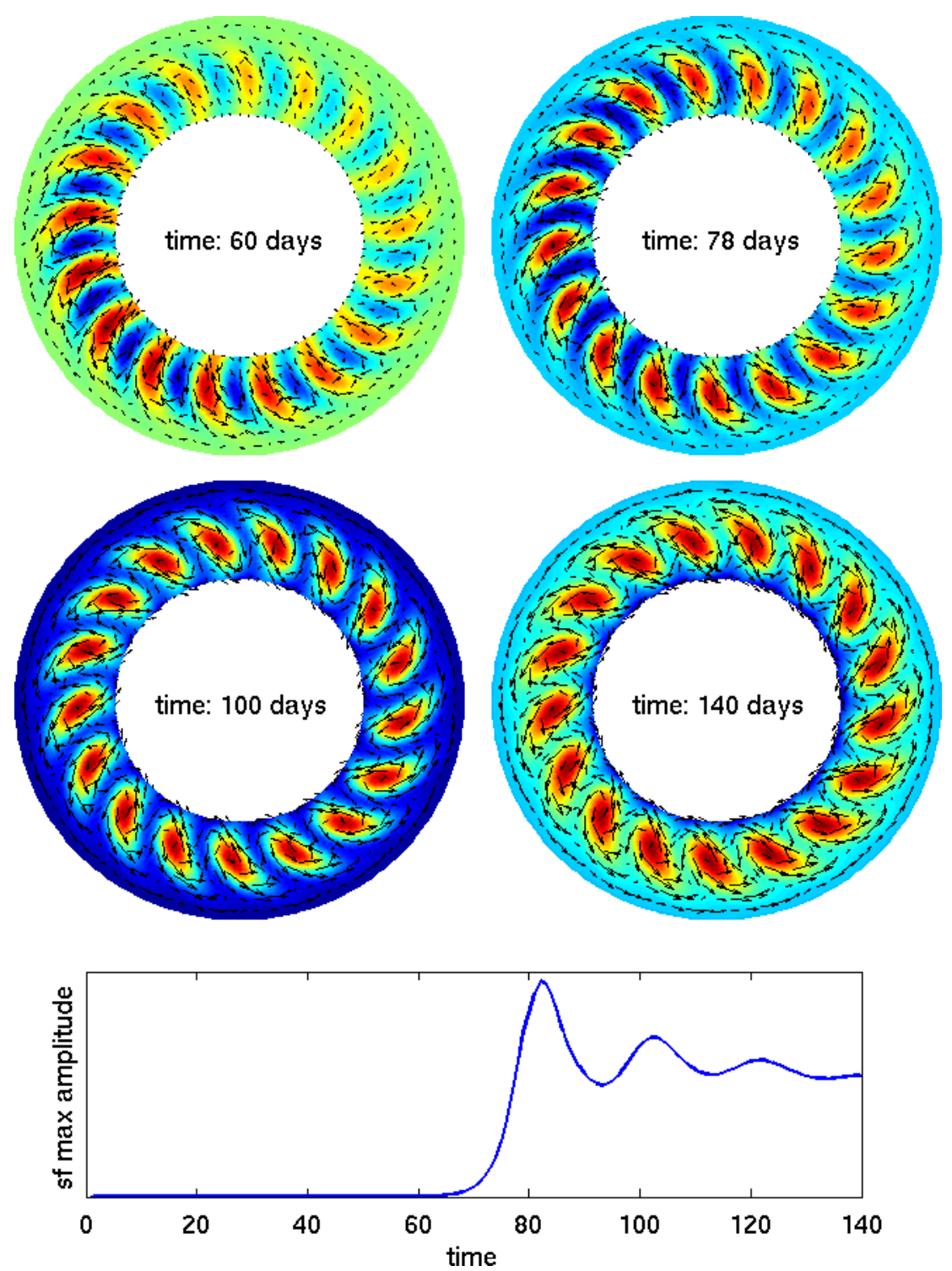

Figure 5.1: The Weakly nonlinear run: $R a=1.5 E 5, E k=4 E-4, \operatorname{Pr}=0.5$. (top) Snapshots of the equatorial streamfunction in time, red is cyclonic rotation and blue is anticyclonic rotation. (bottom) The maximum of the equatorial streamfunction in time. 


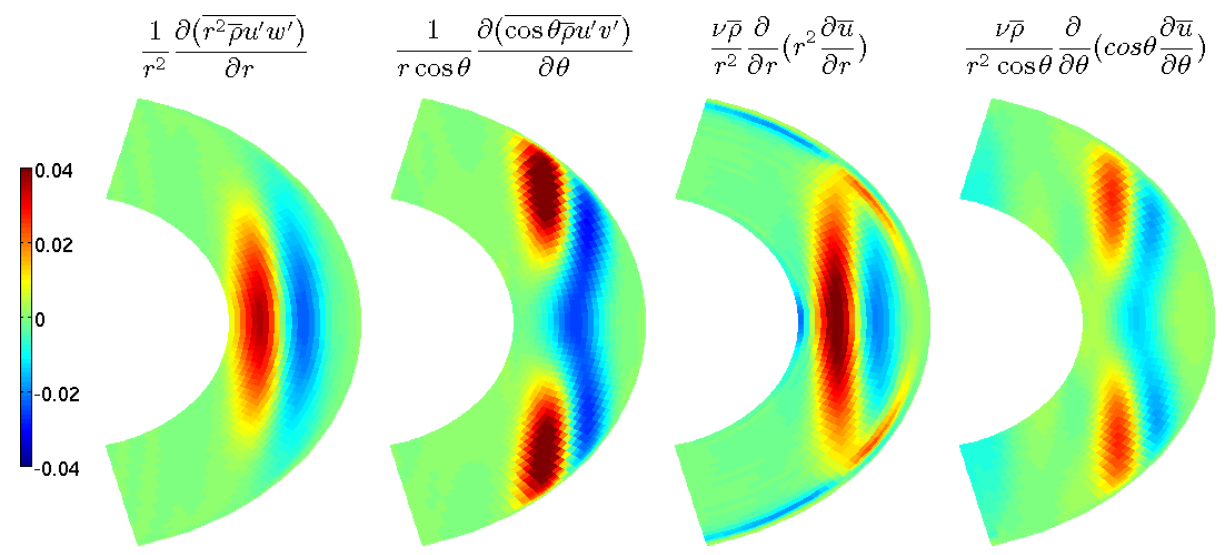

Figure 5.2: The eddy momentum flux divergence and the viscous terms at time $t=100$ (corresponding to Figure 5.1).

the shear and only the cyclones survive. Then the system goes into the second regime which can be seen in Figure 5.1. The cyclones continue to propagate eastward with nearly the same phase velocity as before (see Figure 5.8).

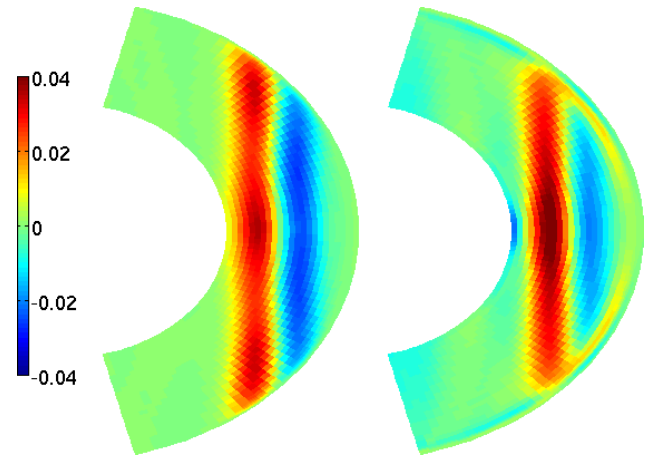

Figure 5.3: The contribution to the eddy momentum flux divergence and the viscous term from fluxes perpendicular to the axis of rotation at time $t=100$ (corresponding to Figure 5.1 and to Figure 5.2).

In this second, weakly nonlinear, regime the amplitude of the flow oscillates until the nonlinearities act to bring the flow to a stable state. This behavior is similar to the behavior we have found in our quasigeostrophic two layer model (chapter 7 ), where once the nonlinear contributions become significant the solution oscillates around a stable state due to the eddy-mean flow interactions (see analysis in section 7.4). In contrast to the quasigeostrophic inviscid model here viscosity also plays a role in inhibiting the growth, and the balance is between the eddy fluxes transferring momentum to the outside to the viscous fluxes which flux momentum inward. Figure 

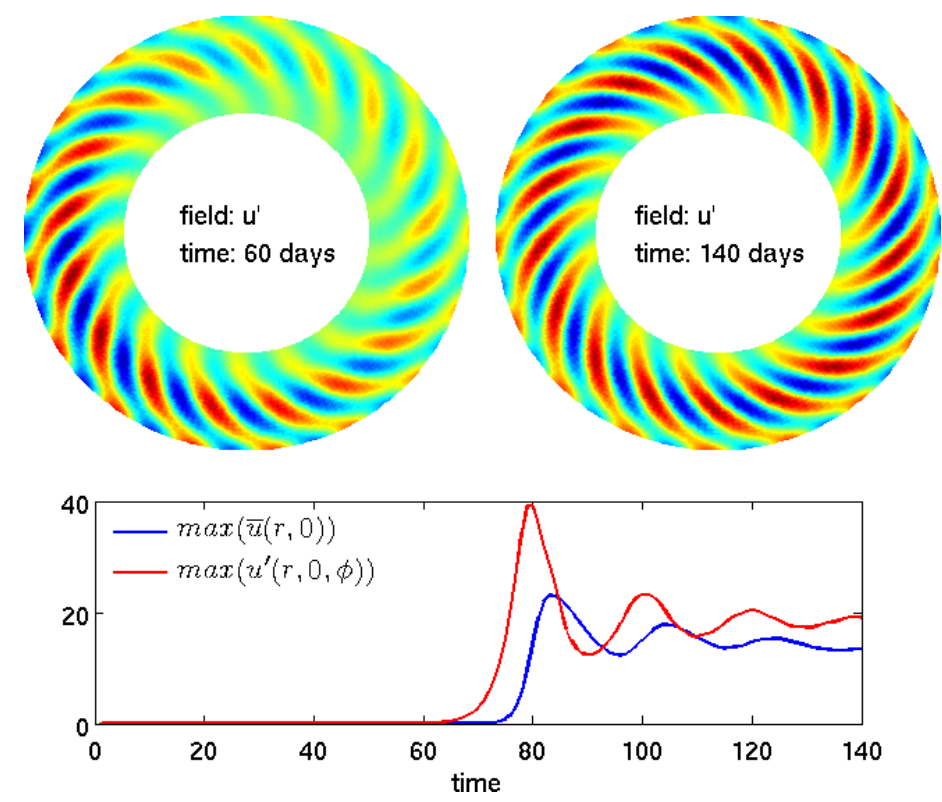

Figure 5.4: The evolution of the $u^{\prime}$ field in the weakly nonlinear run. top: snapshots of the $u^{\prime}$ field on the equatorial plane during the linear (left) and weakly nonlinear (right) stages; bottom: the evolution of the maximum of the $\bar{u}$ and $u^{\prime}$ components of the zonal velocity in time. The radial dependence of $\bar{u}$ is shown in Figure 5.5.

5.2 shows the vertical and meridional contributions to the eddy flux divergence and the viscous terms, which after the instability are close to balance. During the growth stage the viscous contribution is small and the eddy fluxes contribute to the growth of the mean zonal velocity $\frac{\partial \bar{u}}{\partial t}$. Figure 5.3 shows contributions of the eddy flux divergence and the viscous terms, from fluxes acting in the direction perpendicular to the axes of rotation.

It is useful to look at the zonal velocity during this instability and transition between the linear and nonlinear regimes. We divide the zonal velocity into two parts, the zonal mean and the part not containing the zonal mean so that

$$
u=\bar{u}(r, \theta)+u^{\prime}(r, \theta, \phi) .
$$

Figure 5.4 (bottom) shows that the growth of $u^{\prime}$, and with it the outward flux of angular momentum, precedes the growth in $\bar{u}$. Therefore it is the flux of angular momentum outward which contributes to the development of the mean zonal velocity $\bar{u}$. The amplitude of the mean velocity always follows the behavior of the zonally varying component meaning that the outward flux of angular momentum is causing 
the development of the zonally averaged component and consequentially the shear. Once the shear is developed the anticyclonic spiraling vortices which were part of the linear solution disappear and only vortices in the direction of the shear survive. These cyclones are still tilt eastward being in balance between the eddy and the viscous fluxes. Figure 5.4 (top) shows the structure of $u^{\prime}$ both during the linear stage and the nonlinear stage, and in both cases the structure is similar (only with different amplitudes and a overlaying lower mode in the initial stage) and again consistent with the linear calculations of Zhang and Schubert (1997). The radial structure of the shear is shown in Figure 5.5 for the stage after the weakly nonlinear system has reached equilibrium. As seen in Figure 5.4 in earlier stages the zonal mean velocity oscillates around this state until reaching the shear which is in balance with the eddy and viscous fluxes.

The structure along the direction of the axis of rotation is consistent with the structure seen in the fully turbulent case (Figure 4.13). The Taylor-Proudman constraint (with the anelastic adjustments - section 4.3), allows small variation in the direction of the axis of rotation and therefore both the initial anticyclones and cyclones, and the later stronger cyclones extend through the planet forming columns. Figure 5.6 shows the streamfunction on conic surfaces at different latitudinal angles for the weakly nonlinear regime at the stage after the instability. The conic surfaces vary in intervals of $10^{\circ}$ in latitude, showing how the cyclones move outward in latitude such that the cyclones are always in equal distance from the axis of rotation, and therefore are perpendicular to the equatorial plane, forming columns.

In this section we have explained the mechanism leading to the superrotation through the flux of angular momentum and the transition of the linear modes. This weakly nonlinear regime allowed us to connect the linear solution as shown analytically by Zhang and Schubert (1997) to the full nonlinear solution we see in the GCM. In the more turbulent cases the modes are not distinct but the general structure with the cyclones on the equatorial plane and columns extending throughout the planet persists. Another question raised by the turbulent model was the mechanism driving the waves seen on the surface of the planet. These waves seen in Figure 5.7 are embedded within the mean equatorial superrotation and have phase lines which are tilted eastward in both north and south hemisphere with a maximum at the equator. The weakly nonlinear model explains this feature, since superimposed on the mean zonal flow (Figure 5.5), there is a contribution to the zonal velocity from the $u^{\prime}$ 


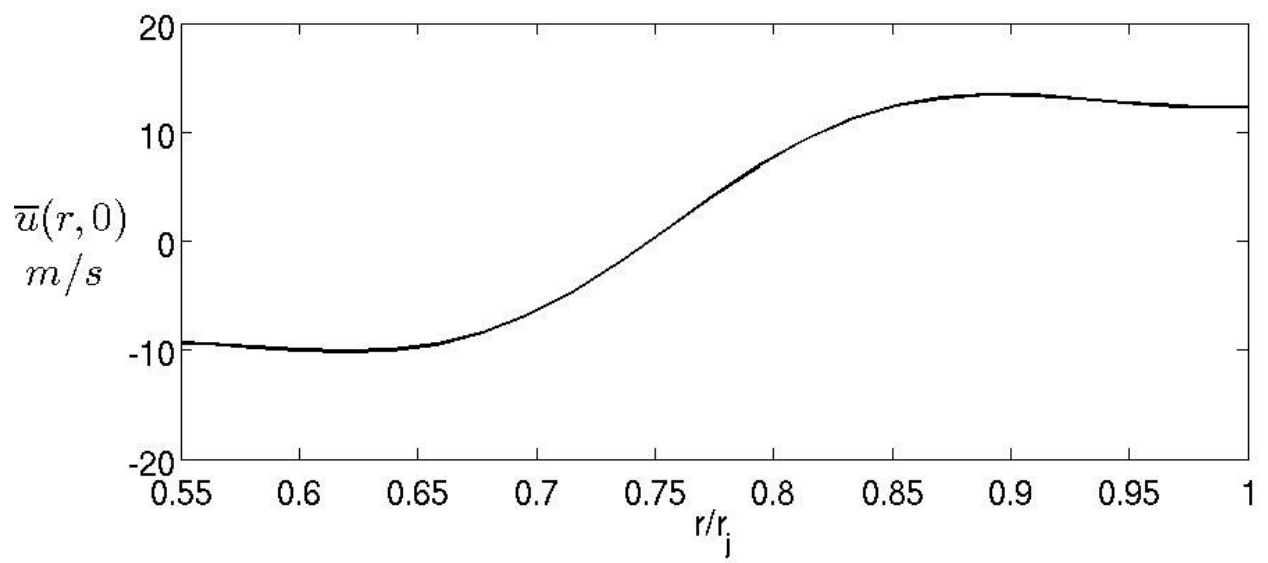

Figure 5.5: The zonal mean component of the zonal velocity on the equatorial plane after the velocity has reached quasi-steady state $(t=140)$ in Figure 5.4.

component (which is not necessarily weak) and associated with columns. Due to the spherical geometry of the surface, and the eastward tilt in the columns, the surface zonal velocity resulting from the columns at the equator ( $u^{\prime}$ in Figure 5.4) would be more eastward than the zonal velocity $\left(u^{\prime}\right)$ at the outcrop of the same column. Therefore the phase line of the column extended to the surface of the sphere has an appearance of a wave with an eastward bend in its phase line. In the turbulent model there are no distinct phase lines but since the columns appear in a turbulent form in the interior, their $u^{\prime}$ component is manifested to the surface with the wave structure appearance. As mentioned in chapter 1, waves with a similar appearance with curved phase line embedded in the superrotation have been observed on Jupiter. These waves had a smaller latitudinal extent but as we will show in section 6.1 the latitudinal extent is affected by the vertical extent of the model. Therefore we propose that this might be a plausible mechanism for the waves although the wavelength of the observed waves is less than the resolution of our model. Note that for high Rayleigh number experiments these phase lines become less apparent. In addition since $u^{\prime}$ is strongest radially (ignoring the anelastic effect for this argument) towards the center of the column, then the $u^{\prime}$ component is strongest at the outcrop of the columns to the surface, giving the appearance of stronger jets at mid latitudes. Both weakly nonlinear and fully turbulent surface zonal velocities can be seen in Figure 5.7.

The existence of the waves in the fully nonlinear case shows that the same general mechanism exists in the fully turbulent (higher Reynolds number and/or lower Ekman number) cases. In chapter 6 we discuss the sensitivity to these parameters and 

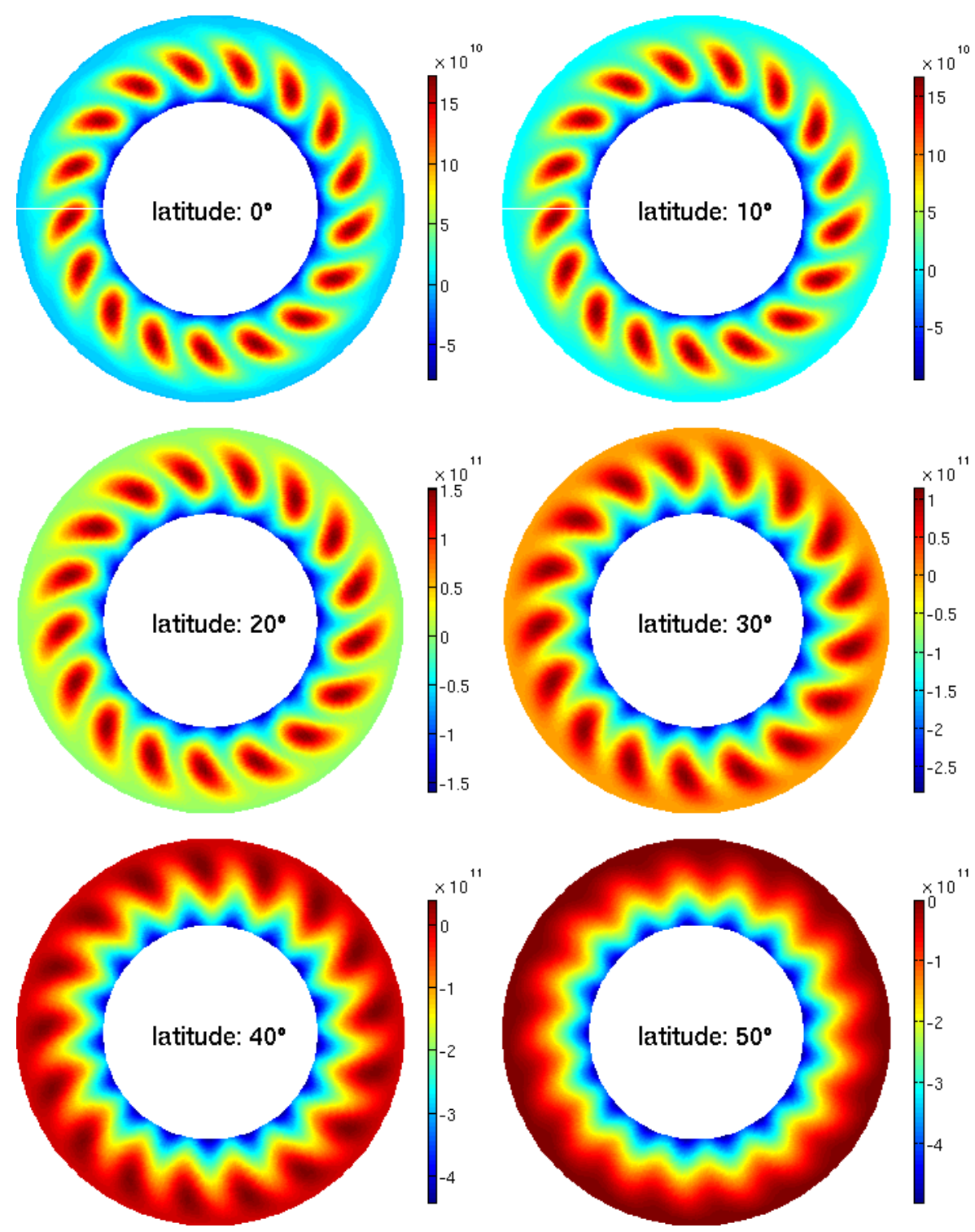

Figure 5.6: The 2D streamfunction on conic surfaces of constant latitudinal angle. Snapshots correspond to $t=140$ days in Figure 5.1 and show that the cyclones seen in the equatorial plane are cyclonic columns extending through the sphere. 

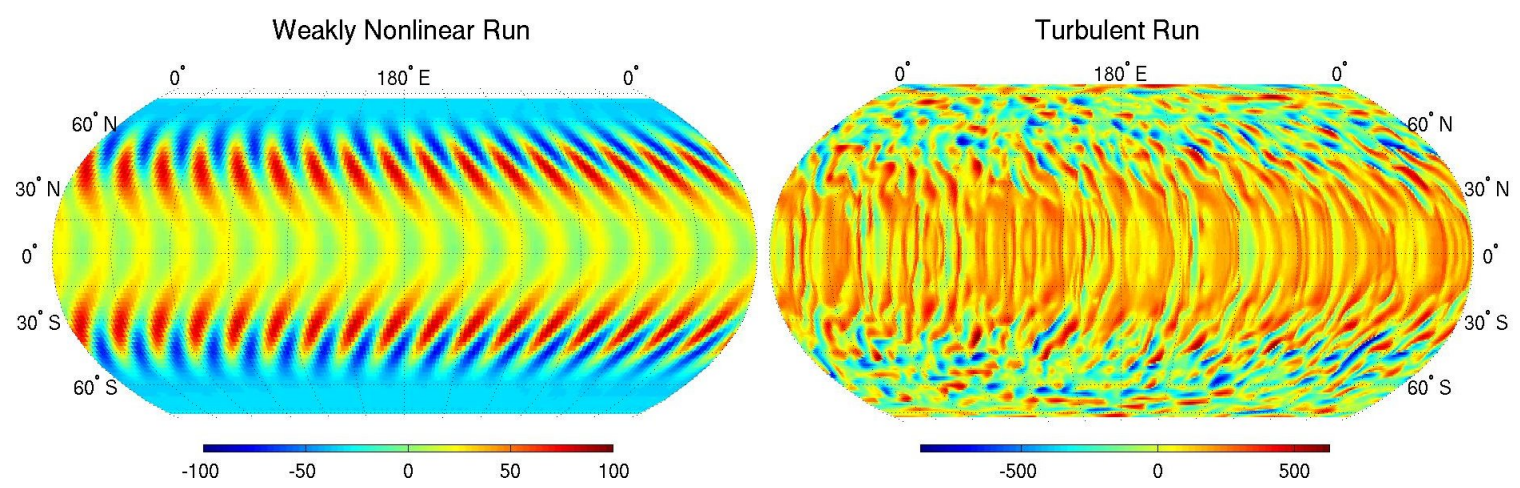

Figure 5.7: Waves embedded in the equatorial surface zonal velocity $(\mathrm{m} / \mathrm{s})$ for the weakly nonlinear case $(R a=, E k=, P r=10)$, and a fully turbulent case $(R a=$ $3 E 6, E k=, 1.5 E-5 \operatorname{Pr}=10)$.

show that for lower Ekman numbers the flow develops more columns and therefore the equatorial superrotation appears smoother. We find also that, when going from a $2^{\circ}$ resolution run to a $1^{\circ}$ resolution run, the latitudinal extent of the equatorial superrotation is reduced. We emphasize that the waves that appear on the surface are superimposed on an eastward zonal mean velocity.

We have explained therefore the mechanism for the equatorial superrotation based on the weakly nonlinear runs. This mechanism however relies on the tilting of the columns for the outward flux of angular momentum, and indirectly on the propagation of the perturbation eastward. In the next sections we discuss these processes in more detail using different models. We begin with a simple model to understand the mechanism for the eastward propagation of the columns.

\subsection{Single Column Barotropic Model}

In order to understand the dynamics of the columns we see in the turbulent flow we turn to a much simpler model. Since we have shown in chapter 4 that the interior is close to barotropic, and the flow is aligned with the rotation axis, a natural system in which to describe a single column model will be a barotropic system in cylindrical coordinates. We follow a similar derivation done by Ingersoll and Pollard (1982) where they have a scale separation between the scale of the columns and the size of the domain. Rewriting $(2.8-2.10)$ in cylindrical coordinates (which aligns with the spherical system at $\theta=0$ with replacing the meridional coordinate with $z$ and noting 
that now $\bar{\rho}=\bar{\rho}(r, z))$ gives,

$$
\begin{aligned}
\frac{D u}{D t}-\frac{u w}{r}-2 \Omega w & =-\frac{1}{r} \frac{\partial \Phi}{\partial \phi} \\
\frac{D v}{D t} & =-\frac{\partial \Phi}{\partial z} \\
\frac{D w}{D t}+\frac{u^{2}}{r}+2 \Omega u & =-\frac{\partial \Phi}{\partial r}
\end{aligned}
$$

Note that we are not using the traditional cylindrical coordinates, to be consistent with our previous notation, so that $u$ is the azimuthal velocity, $w$ is the radial velocity and only $v$ is redefined as $v=\frac{d z}{d t}$ (but locally on the equatorial plane coincides with the spherical form so that $d v=r d \theta$ ). In this system the Coriolis terms parallel to the rotation axis vanish and we have used the anelastic potential as defined in (2.23). The mass equation 2.5 gives

$$
\frac{\bar{\rho}}{r}\left(\frac{\partial u}{\partial \phi}+\frac{\partial(r w)}{\partial r}\right)+w \frac{\partial \bar{\rho}}{\partial r}+\frac{\partial(\bar{\rho} v)}{\partial z}=0 .
$$

We scale time by the advective time scale, but where there is a length scale separation between the local length $L$ and the domain radius $r_{0}$ so that $L \ll r_{0}$. Then for a small Rossby number to the highest order when cross differentiating (5.2) and (5.4), subtracting them and adding (5.5) we get that

$$
\frac{D}{D t}\left[\frac{\partial w}{\partial \phi}-\frac{\partial(r u)}{\partial r}\right]+\frac{2 \Omega r}{\bar{\rho}}\left(w \frac{\partial \bar{\rho}}{\partial r}+\frac{\partial(\bar{\rho} v)}{\partial z}\right)=0 .
$$

The term is the square brackets is the vorticity, and the terms on the right are the contributions to the vorticity from stretching and the variations in the mean density. This expression therefore will describe the vorticity of a single column within the sphere as shown in section 5.1. This system resembles a quasigeostrophic system, although (5.3) is different. Ingersoll and Pollard (1982) show this equivalence using the ratio between ratio of cylinder and the ratio of the sphere as the small parameter in analogy to the Rossby number in QG.

At the limit of small Rossby number the quasigeostrophic equivalent scaling of (5.3), and (5.4) will give to the highest order

$$
w=\frac{1}{2 \Omega r} \frac{\partial \Phi}{\partial \phi} \quad, \quad u=-\frac{1}{2 \Omega} \frac{\partial \Phi}{\partial r}
$$



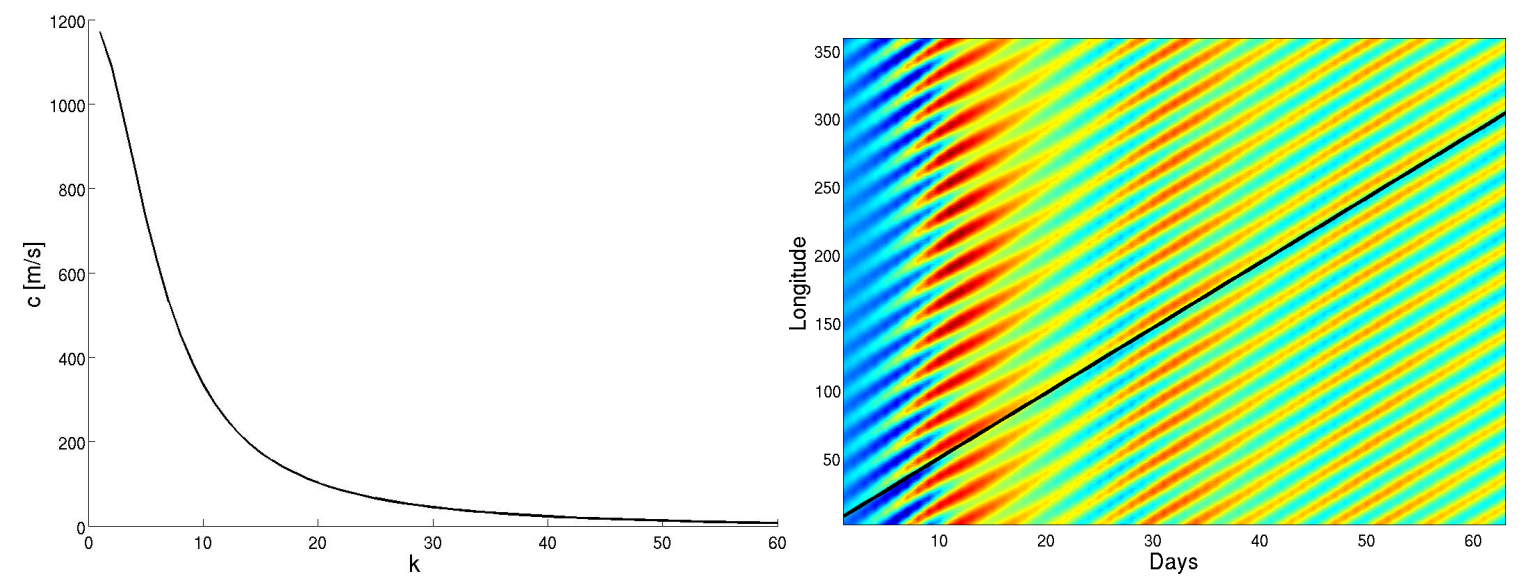

Figure 5.8: left: the relation $c(k)$ from eq. (5.14), and the Hovmoller diagram from the weakly nonlinear run with the phase speed of $51 \frac{\mathrm{m}}{\mathrm{s}}$ superimposed.

so that the anelastic potential is the geostrophic streamfunction. We assume a basic state as shown in section 5.1 where the flow develops a basic state $\bar{u}(r)$ and a perturbation which can be described by a streamfunction (5.7)

$$
\Phi=2 \Omega \psi(r, z) e^{i k(\phi-\sigma t)},
$$

where $k$ is the zonal wave number and $\sigma$ is the frequency. Following (5.7) the velocities therefore become

$$
\begin{aligned}
u & =\bar{u}(r)-\frac{d \psi}{d r} e^{i k(\phi-\sigma t)} \\
w & =\frac{i k}{r} \psi e^{i k(\phi-\sigma t)} \\
v & =\zeta(z) e^{i k(\phi-\sigma t)}
\end{aligned}
$$

Then to the highest order assuming the mean flow is larger then the perturbation, (5.6) and (5.3) become

$$
\begin{aligned}
(\bar{u}-c)\left[\frac{1}{r} \frac{\partial}{\partial r}\left(r \frac{\partial \psi}{\partial r}\right)-\frac{k^{2}}{r^{2}} \psi\right]+\left[\frac{2 \Omega}{\bar{\rho}} \frac{\partial \bar{\rho}}{\partial r}-\frac{1}{r} \frac{\partial}{\partial r}\left(r \frac{\partial \bar{u}}{\partial r}\right)\right] \psi & =\frac{2 \Omega r}{\bar{\rho}} \frac{\partial(\bar{\rho} \zeta)}{\partial z}(5.12) \\
(\bar{u}-c) k^{2} \zeta & =\frac{\partial \psi}{\partial z}
\end{aligned}
$$

We turn now to our numerical simulations where we have seen that on the equatorial plane the meridional variations in the streamfunction are small (Figures 3.9, and 
4.13), and therefore to make this system separable we assume that the right hand side terms containing the variation in the meridional direction are negligible. From (5.13) this is similar to assuming the zonal wavelength is small compared to the radial wavelength, which leads to having the streamfunction $\psi$ independent on $z$ so that $\psi=\psi(r)$ only. Thus the flow on the equatorial plane can be described as a $2 \mathrm{D}$ streamfunction. We have therefore set an eigenvalue problem which can be solved to find the phase speed. The phase speed will describe the propagation of the columns on the equatorial plane which we have seen in the previous section. Alternatively, we can do a local estimate for the phase speed by using the local numeric values we have for the shear and streamfunction at the radial location of the columns. We then get a local estimate for the phase speed as function of the zonal wavenumber

$$
\left.c\right|_{r_{c}}=\frac{\left.\left[\left.\frac{2 \Omega}{\bar{\rho}} \frac{\partial \bar{\rho}}{\partial r}\right|_{r_{c}}-\left.\frac{1}{r} \frac{\partial}{\partial r}\left(r \frac{\partial \bar{u}}{\partial r}\right)\right|_{r_{c}}\right] \psi\right|_{r_{c}}+\left.\bar{u}\right|_{r_{c}}\left[\left.\frac{1}{r} \frac{\partial}{\partial r}\left(r \frac{\partial \psi}{\partial r}\right)\right|_{r_{c}}-\left.\frac{k^{2}}{r^{2}} \psi\right|_{r_{c}}\right]}{\left.\frac{1}{r} \frac{\partial}{\partial r}\left(r \frac{\partial \psi}{\partial r}\right)\right|_{r_{c}}-\left.\frac{k^{2}}{r^{2}} \psi\right|_{r_{c}}}
$$

where $r_{c}$ denotes the radial location of the maximum of the zonally averaged equatorial streamfunction (the radial location of the columns). In Figure 5.8 we show $c(k)$ for the weakly nonlinear run presented in section 5.1 calculated in this method. We find that this gives an inverse relation between the zonal wavenumber (number of columns), and the phase speed. Figure 5.8 shows a Hovmoller diagram of the equatorial streamfunction around the radial distance of the columns. We see that the columns propagate eastward at a phase speed of $51 \mathrm{~m} / \mathrm{s}$. Using this value in Figure 5.8 corresponds to a wave number of $k=28$. The number of columns in the model is 18 however given the rough approximation of this model (mostly assuming inviscid dynamics) it might be a right ball-park number. More importantly, (5.12) predicts an eastward propagation of the columns. It is important to note the similarity between (5.12) and the barotropic stability equation on a beta plane (e.g. Pedlosky, 1987), where the term of the radial derivative of the mean density acts as the effective $\beta$. Multiplying (5.12) by $\bar{\rho}$, and integrating in the $z$ direction (assuming as discussed above that the streamfunction is independent of $z$ ) between the places the columns intersect with the surrounding sphere denoted by $-h$ and $h$ gives

$$
(\bar{u}-c)\left[\frac{1}{r} \frac{\partial}{\partial r}\left(r \frac{\partial \psi}{\partial r}\right)-\frac{k^{2}}{r^{2}} \psi\right]+\left[B-\frac{1}{r} \frac{\partial}{\partial r}\left(r \frac{\partial \bar{u}}{\partial r}\right)\right] \psi=0
$$




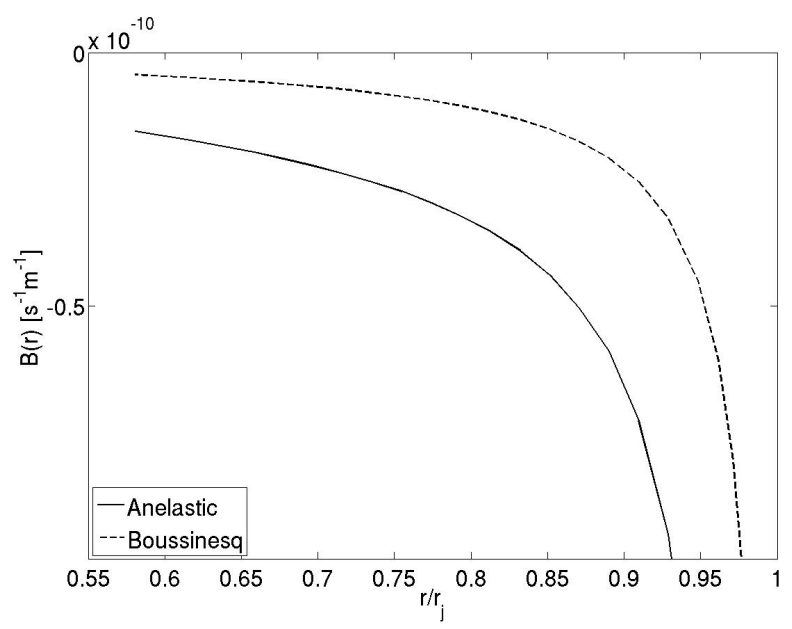

Figure 5.9: The effective $\beta B(r)$ (5.16) as a function of radius for both Anelastic and Boussinesq cases.

where

$$
B(r)=\frac{2 \Omega}{M} \frac{d M}{d r}
$$

and

$$
M=\int_{-h}^{h} \bar{\rho} d h .
$$

$B(r)$ therefore is the effective $\beta$, and is a function of the radial distance in the sphere. For a Boussinesq fluid $M$ would simply grow as $r$ becomes smaller due to the spherical boundaries of the sphere. In the anelastic case $M$ will have a more complex behavior due to the effect of the boundaries, and the radial dependence of $\bar{\rho}$ itself. This is demonstrated in Figure 5.9. In both cases since $\frac{d M}{d r}$ is negative the effective $\beta$ in the interior of a sphere would then be negative. Ingersoll and Pollard (1982) use this expression do derive an alternative barotropic stability criterion which we come back to in chapter 7 .

To understand the effect of the negative beta intuitively, one can think about the stretching of a column of fluid as it is moved closer to the axis of rotation. While in the standard spherical shell such a column will shrink in length as it is moving poleward, a column in the interior will stretch. In the thin spherical shell, this effect 

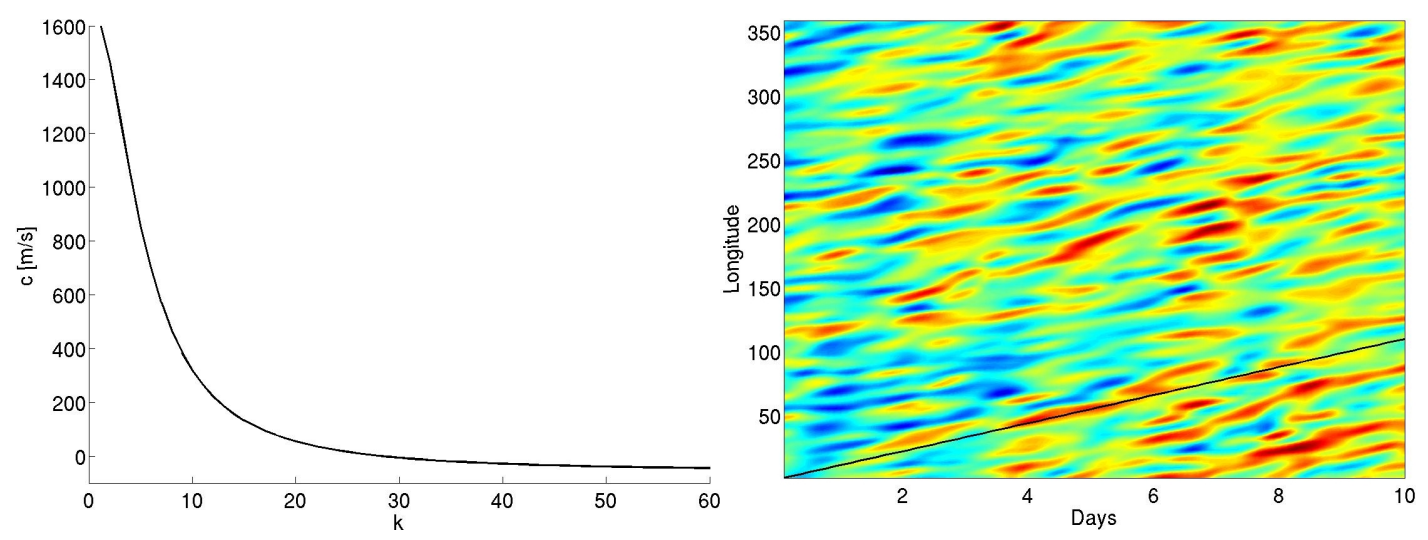

Figure 5.10: Left: The relation $c(k)$ for the fully turbulent run (moderate Rayleigh number as in Figures 3.9-3.11 $R a=3 E 6, E k=1.5 E-4, \operatorname{Pr}=10$ ); right: the corresponding Hovmoller diagram with the phase velocity of $c=120 \frac{\mathrm{m}}{\mathrm{s}}$ superimposed with the black line.

is equivalent to a positive planetary vorticity gradient (which in terms of conservation of potential vorticity is equivalent to a bottom slope growing towards the pole in a shallow system). In the deep system the stretching of the columns is equivalent to having a negative planetary vorticity gradient towards the poles. Here therefore, we can think about the effect of $B(r)$ as the background planetary vorticity only with the opposite effect to that of a thin spherical shell. Similarly, a Rossby wave will propagate in the opposite direction. If we go back to Figure 5.1 the set of positive and negative perturbations feels the effect of the planetary vorticity gradient and by conserving potential vorticity on the equatorial plane propagate eastward. The mechanism is similar to that of a Rossby wave except that instead of polar movement causing negative relative vorticity, motion toward the center of the planet (poleward) causes positive relative vorticity and positive phase speed. In chapter 7 we look how baroclinic instability changes in the presence of a negative $\beta$. Figure 5.10 is similar to Figure 5.8 only for a more turbulent case. Again we find that the phase speed of the propagation of the columns is close to the values predicted by (5.14) for the number of columns we find in the numerical model.

We have explained the eastward propagation of the columns and shown an estimate for the number of columns that form. However the mechanism described in section 5.1 would not work if there was no flux of momentum as the columns propagate. Therefore we now turn to another model - 'the annulus model' to understand the reason for this flux, and preference for only cyclonic rotating columns. 

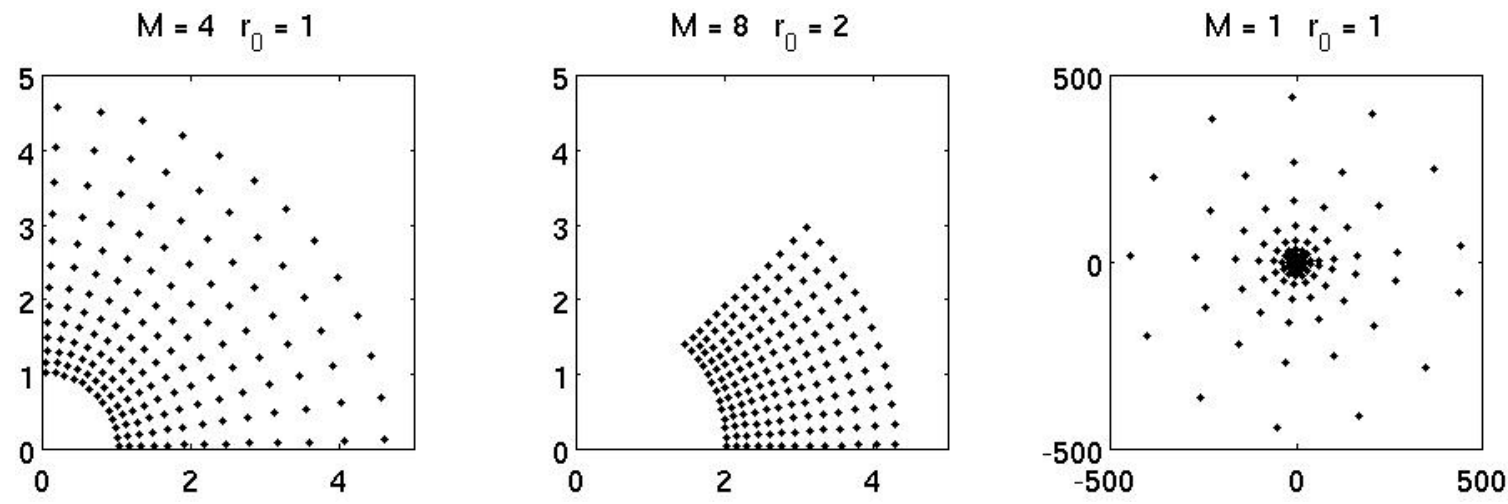

Figure 5.11: Mapping the square $(0,2 \pi)$ to the annulus grid. (left) $M=4, r_{0}=1$, (middle) $M=8, r_{0}=2$, (right) $M=1, r_{0}=1$

\subsection{The Annulus Model}

An important aspect of the process leading to the superrotation which was discussed in the previous sections was the flux of momentum to the outer parts of the sphere due to the spiraling in the linear modes. In this section we use another simplified model to study this process. In order to represent the spherical geometry in a simple channel model, we use a barotropic model with varying height, and use a conformal mapping to map this channel to an annular surface (Mehta, 1998). By this we can represent the beta effect with the variation in the model height (a deeper interior represents a negative planetary vorticity gradient and visa versa). Using a linear slope will approximate a constant beta, a convex slope will have a bigger values of beta towards the outside and a concave slope will have the opposite effect. The mapping of the channel to the annulus gives the proper metric of the sphere's equatorial plane. The model assumes conservation of potential vorticity and the height weighted velocity is nondivergent.

We construct the annulus coordinates by using the following mapping,

$$
\begin{aligned}
r & =r_{0} e^{\frac{y}{M}} \\
\theta & =-\frac{x}{M} \\
Z & =z,
\end{aligned}
$$

which relates the annulus coordinates $(x, y, z)$ to cylindrical coordinates $(r, \Theta, Z)$. Figure 5.11 shows how the Cartesian square between 0 and $2 \pi$ is mapped to the 

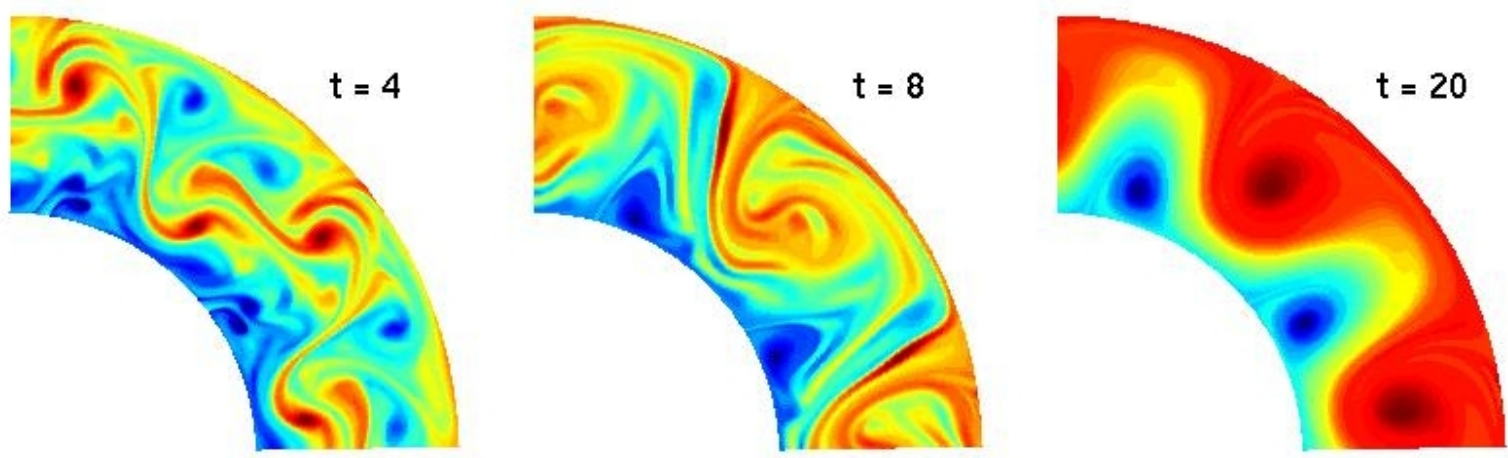

Figure 5.12: Snapshots from a run using the $H(r)$ profile from the full spherical model (equation (5.16) at radius $0.6-0.8)$. The flow forms vortices through an inverse cascade that propagate eastward.

annulus for different values of $M$ and $r_{0}$.

We solve the following system

$$
\begin{aligned}
\frac{D q}{D t} & =0 \\
\nabla \cdot(u H) & =0
\end{aligned}
$$

where $q=\frac{\zeta+f}{H}$ is the potential vorticity, $H$ is the depth of the fluid, $f$ is the Coriolis number, $\zeta$ is the relative vorticity, and the $2 \mathrm{D}$ streamfunction $\psi$ is defined so that

$$
\mathbf{u} H=\nabla \times \psi
$$

The streamfunction is therefore related to the vorticity by

$$
\zeta=\nabla \cdot \frac{1}{H} \nabla \psi
$$

We define $\mathbf{U}=\mathbf{u} H$ so that (5.21), and (5.22) combine to give

$$
\frac{\partial q}{\partial t}+\frac{1}{H} \nabla \cdot(\mathbf{U} q)=0,
$$

or equivalently

$$
\frac{\partial \zeta}{\partial t}+\nabla \cdot(\mathbf{U} q)=0 .
$$


Next we calculate the scale factors necessary to transform the Cartesian channel model to the annulus coordinate system. Inverting equations (5.18 - 5.20), and defining a Cartesian system $(\xi, \eta, \varsigma)$ gives the following transformation from a Cartesian grid to an annular one

$$
\begin{aligned}
& y=\frac{1}{2} M \ln \left(\frac{\xi^{2}+\eta^{2}}{r_{0}^{2}}\right) \\
& x=-M \tan ^{-1}\left(\frac{\eta}{\xi}\right) \\
& z=\varsigma,
\end{aligned}
$$

so that the Jacobian giving the area scaling factor from the Cartesian to the annular system is

$$
\frac{\partial(x, y)}{\partial(\xi, \eta)}=\frac{M^{2}}{r^{2}}
$$

Therefore this will be the factor scaling the Jacobian term in equation (5.26), when transforming the Cartesian system to the annular one. Similarly using (5.24) both the divergence and the gradient operator contribute a factor $\frac{M}{r}$, so that the vorticity equation with the transformation factors becomes

$$
\frac{M^{2}}{r^{2}} \frac{\partial \zeta^{\prime}}{\partial t}+\frac{M^{2}}{r^{2}} \nabla \cdot\left(\mathbf{U}^{\prime} q^{\prime}\right)=0
$$

and the potential vorticity is

$$
q^{\prime}=\frac{\frac{M^{2}}{r^{2}} \zeta^{\prime}+f}{H}
$$

Therefore we can calculate the change in vorticity by solving for the potential vorticity flux. The scaling factor will come only in the potential vorticity.

The profile of $H(r)$ would therefore control the nature of the dynamics on the annulus plane. The case that would represent our full numerical model would be to take the $H$ profile given by (5.16). In Figure 5.12 we show the resulting flow when beginning from an initial high modal perturbation, and a zero mean vorticity. Due to the negative beta effect the flow develops a eastward propagation. Opposed to the convective model, since the model is not continuously forced by small scale 

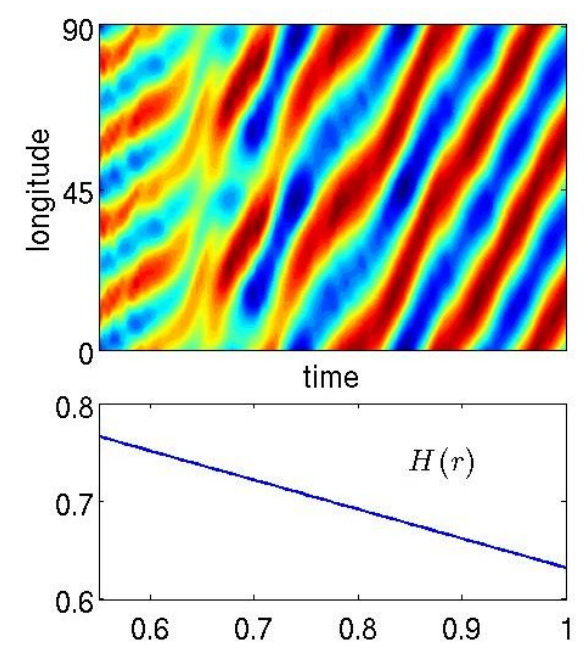

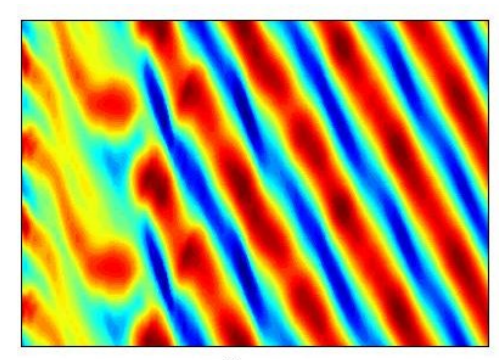

time

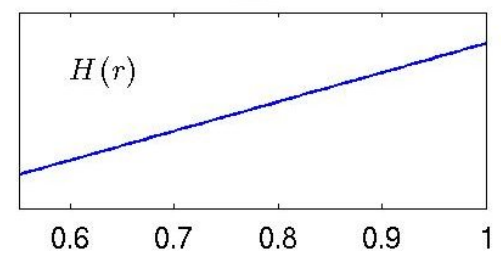

Figure 5.13: Hovmoller diagrams of the perturbation potential vorticity for cases of a positive and negative linear slope in $H(r)$. The positive slope (shallow fluid at smaller radius - positive $\beta$ ) develops westward propagation, and the case that simulates a deep sphere (deeper fluid at smaller radius - negative $\beta$ ) propagates eastward.

convection it develops an inverse energy cascade and forms large cyclonic vortices. Note that the reason for the formation of these vortices is different from the columnar vortices in the full model since there a mean shear develops due to the outward flux of angular momentum. In Figure 5.13 we show Hovmoller plots from two experiments with a linear slope of $H(r)$, where one slope is positive and the other is negative. The Hovmoller plots show the opposite direction of propagation of the vortices in both cases. Where the slope makes a shallower fluid in the interior of the annulus (equivalent to positive beta) the vortices develop a westward propagation.

\subsubsection{Solving for the Eigenmodes}

We now turn to look to the reason the modes seen in Figure 5.1 are spiraling. We should make first a distinction between the convection model and the annulus model. In the convection model energy is continuously fluxed outward, accelerating the superrotation, and in steady state dissipated by the viscosity. The annulus model on the other hand evolves from a given initial condition, and modes are not growing. Therefore when looking at the eigenmodes in the annulus model they will have a finite and real phase speed. Then, the only way the eigenmodes can be complex, and thus be tilted in respect to the radial direction, will be in the presence of friction. 

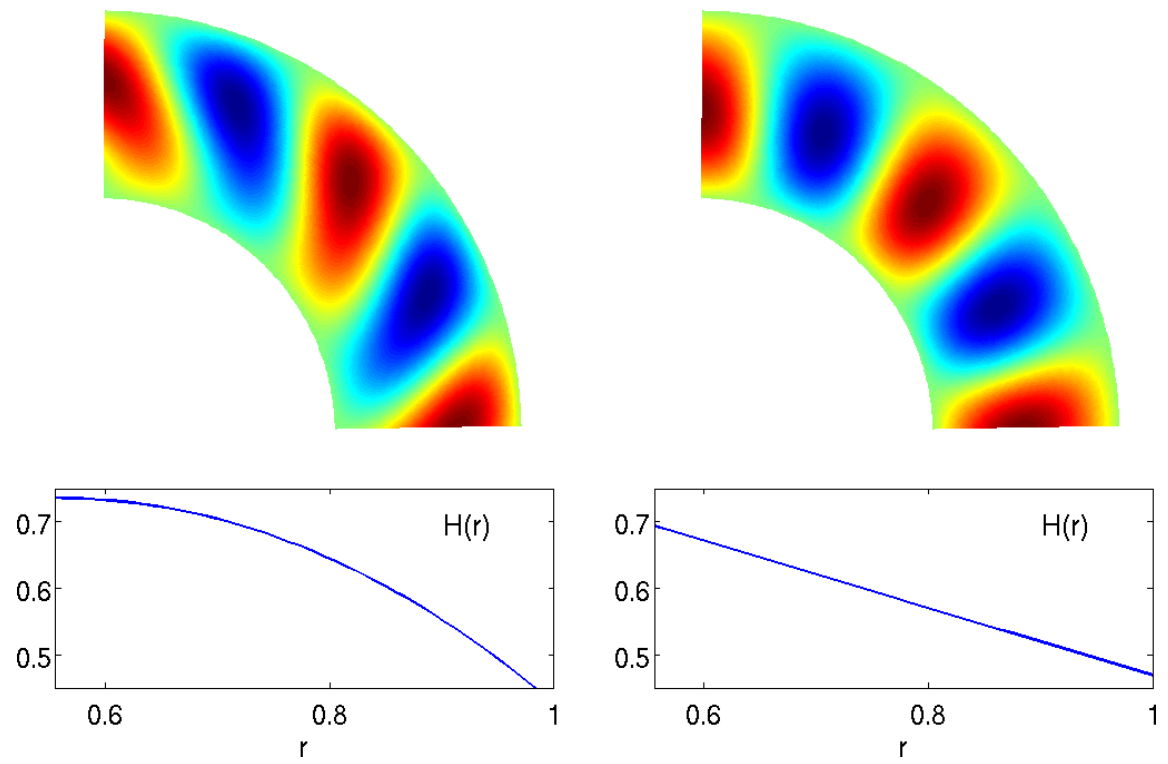

Figure 5.14: Eigenmodes for cases of linear slope in $H(r)$ and a curvature slope in $H(r)$.

Linear models showing the spiraling in the linear modes (Zhang and Schubert, 1997) have also had finite Prandtl numbers.

We solve therefore for the same system presented in $(5.21,5.22)$ but add a constant viscosity so that

$$
\frac{\partial \zeta}{\partial t}+U \cdot \nabla q=\nu \nabla^{2} \zeta .
$$

We solve now for the eigenmodes by assuming a solution for the vorticity of the form

$$
\zeta=e^{i k(x-c t)} z_{0}(y),
$$

where $x$ and $y$ are defined in $(5.27,5.28), k$ is the azimuthal wave number and $c$ is the phase speed. Plugging (5.34) in (5.33), and defining an operator $M$ so that

$$
\zeta=\nabla \cdot \frac{1}{H} \nabla \psi \equiv M \psi,
$$

gives an equation of the form

$$
\left[M^{-1} \frac{\partial q}{\partial y}-\frac{i \nu}{k}\left(-k^{2}+\frac{\partial}{\partial y} \frac{1}{H} \frac{\partial}{\partial y}\right)\right] z_{0}=-c z_{0},
$$


where $z_{0}$ are the eigenvectors and $c$ is the eigenvalue. Therefore in the absence of friction, and if the linear modes are not growing the eigenmodes will be real (only depend on $r$ ) and there will be no tilting or spiraling of the $z_{0}$ eigenmodes. However, the presence of a finite viscosity still does not guarantee that the eigenmodes will be spiraling.

We should separate the issue of tilting of the modes from the issue of eastward spiraling of the modes. First, in the convection model due to the outward flux of energy, theoretically modes may develop a tilt (and therefore a correlation between zonal and vertical velocity directions), because of the direction of energy propagation, leading to an outward flux of angular momentum. However, in the lack of spiraling (without considering boundary effects) this flux would be nondivergent and therefore will not accelerate a zonal superrotating flow as demonstrated in (4.24). In the convection model we find that due to the inherent radial variation of the planetary vorticity, due to the sphericity, it is difficult to separate the issue of tilting from that of spiraling (spiraling of the columns includes tilting). In the annulus model since we have no convective flux, we can not separate these issues either since without variation of planetary vorticity we do not develop neither spiraling nor tilting.

In order to see this in the annulus model, we show in Figure 5.14 the eigenmodes for two cases of equal parameters, but one with a linear slope and one with a curved slope. The linear slope is equivalent to a constant $\beta$, and the curved one is equivalent to a varying $\beta$. Only the curved one develops spiraling in the direction of the eigenmodes in respect to the radial axis. Therefore the spiraling of the modes is related to the radial variation of planetary vorticity.

A semi-analogous case (considering more the issue of tilting and not the angular momentum flux) which may resemble more the convection case with energy continuously fluxed outward, is a case of ocean waves approaching a sloping beach and being refracted due to the variation in the ocean bottom slope. The slope in the bathymetry will result in the local variation in the phase speed $c=(g H)^{\frac{1}{2}}$, and cause a refraction in the orientation of the crest resulting in the waves approaching the coast parallel to the shore line. In the case of the convection model the restoring force is the planetary vorticity rather than gravity, and the cause of the spiraling is the variation in planetary vorticity gradient rather than the surface slope, but the analogy is in the tilting of the wave guide.

To analyze the issue of spiraling further using the annulus model, we define the 


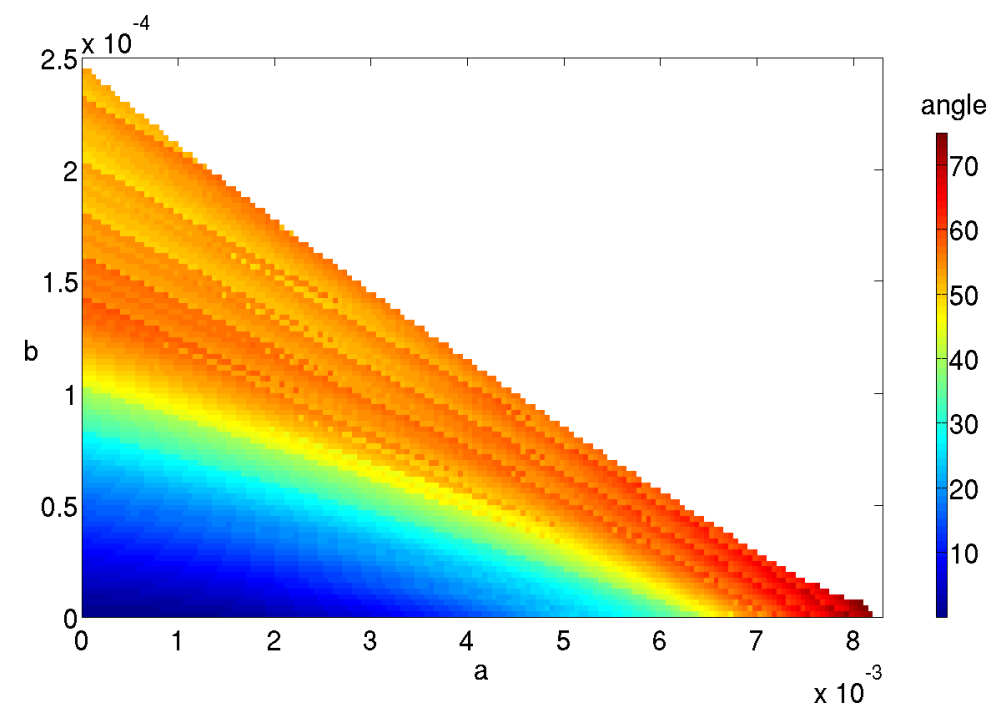

Figure 5.15: The angle of the spiraling of the eigenmodes as a function of the linear and the quadratic coefficients of the depth $H(r)$. Angle is given in degrees eastward of a line along the radius.

height of the fluid layer as

$$
H(r)=H_{0}-a r-b r^{2}
$$

where $H_{0}, a$, and $b$ are constants. We then solve for the eigenmodes for a series of experiments where we vary the linear and quadratic coefficients $a$, and $b$. The curvature grows for larger $b$ values. Figure 5.15 shows the results for a series of experiments where $a$ and $b$ vary for the complete range of positive depths. The angle of spiraling (zero is no spiraling) is calculated using the ratio of the real and imaginary parts of $z_{0}$ at the radial point where the imaginary part is maximum for the fastest growing mode. We see that as the curvature becomes stronger, larger spiraling develops which in the spherical convection model will be associated with an angular momentum eddy flux divergence and the formation of superrotation. Note that even cases of $b=0$ may effectively have some curvature because the way the gradients are defined in (5.35). 


\section{Chapter 6}

\section{Model Sensitivity Analysis}

In the results presented so far we have used one specific geometric configuration of the model, extending radially from the surface to 0.55 of the planetary radius, and several nondimensional parameter configurations ranging from weakly nonlinear runs to more turbulent runs. In this chapter we systematically vary each of these parameters, namely the Rayleigh, Ekman and Prandtl numbers, to study their effect on the various features studied in previous chapters. In addition we study the effect of varying the geometric configuration of the model ranging from a thin spherical shell to nearly a full sphere.

To preform a systematic assessment of these parameters, due to the long computational time of the $1^{\circ}$ resolution runs presented in the previous sections, we use a lower resolution configuration of $2^{\circ}$ resolution latitudinally and longitudinally and a pressure ratio of 2 between vertical levels. When comparing this configuration to the $1^{\circ}$ configuration we find that the overall structure of the circulation (equatorial superrotation, number of columns, etc.) does not change significantly. However, the small scale features at high latitudes disappear, and in particular the equatorial superrotation is on average $5^{\circ}$ wider latitudinally. Nevertheless we find these experiments useful in studying the parameter regime of the model, and we point to the differences due to resolution in the discussion.

Beyond the magnitude of the forcing, we have made in chapter 2 assumptions on the vertical profile of the forcing. Here, we study the effect of the continuous forcing assumption made in section 2.5 by looking at a different type of forcing, and discuss the effects solar forcing can have on the convectively driven circulation. In addition we give examples of interesting solutions we find during spin-up that are unstable, 
and therefore have not been presented earlier when discussing the statistically steady state solutions.

\subsection{From a Spherical Shell to a Full Sphere}

In previous chapters we have used a configuration in which the model extends radially down to 0.55 the radius of the planet ( $\sim 12$ Mbar). In chapter 3 we discussed this choice which is deeper than what has been done in the previous Boussinesq models, and due to the complexity of the interior thermodynamics and the resulting MHD effects, might even be beyond the relevant regime for Jupiter (although this is controversial). However, the goal is to study a system where the vertical scales are comparable to the horizontal ones, and to be in a regime where the location of the bottom boundary does not put constraints on the dynamics. In this section we will vary the depth of the model and study its effects.

We begin therefore with varying the geometry of the model by moving the location of the bottom boundary. Since both Rayleigh and Taylor numbers depend greatly on a depth scale (which we take to be the vertical extent of the model), then instead of holding the Rayleigh and Taylor number constant in these experiments we hold directly the viscosity and heat flux constant. Perhaps the best parameter to keep constant in such experiments would be the ratio $\chi=\frac{T a \cdot P r}{R a}$ which was shown in section 4.5 to characterize the dynamics and has the $H^{4}$ dependence of the Rayleigh and Taylor number cancel but still has a $H^{\frac{1}{2}}$ dependence on the total depth. To keep the experiments simple we held constant the viscosity and heat flux directly.

In Figure 6.1 we show the zonally averaged zonal velocity for a meridional section (similar to Figures 3.9) for a series of experiments where we vary the location of the bottom boundary. We denote by $D$ the ratio $D \equiv \frac{r_{t}-r_{b}}{r_{t}}$ where $r_{t}$ is the top boundary and $r_{b}$ is the bottom boundary. The range of the experiments is from a relatively thin shell (still has three orders of magnitude variation in density) occupying $10 \%$ of the radius $(D=0.1)$, to almost a full sphere occupying $93 \%$ of the radius $(D=0.93)$. For numerical reasons we can not reach a singular point in the interior, but higher ratios are achievable with smaller time stepping. Jupiter is believed (Guillot et al., 2004) to have a solid core occupying the inner $10 \%$ of the planet radius. This series of plots shows that the superrotation is robust for most runs, though for the runs with small aspect ratios the superrotation has a smaller latitudinal extent. For the 


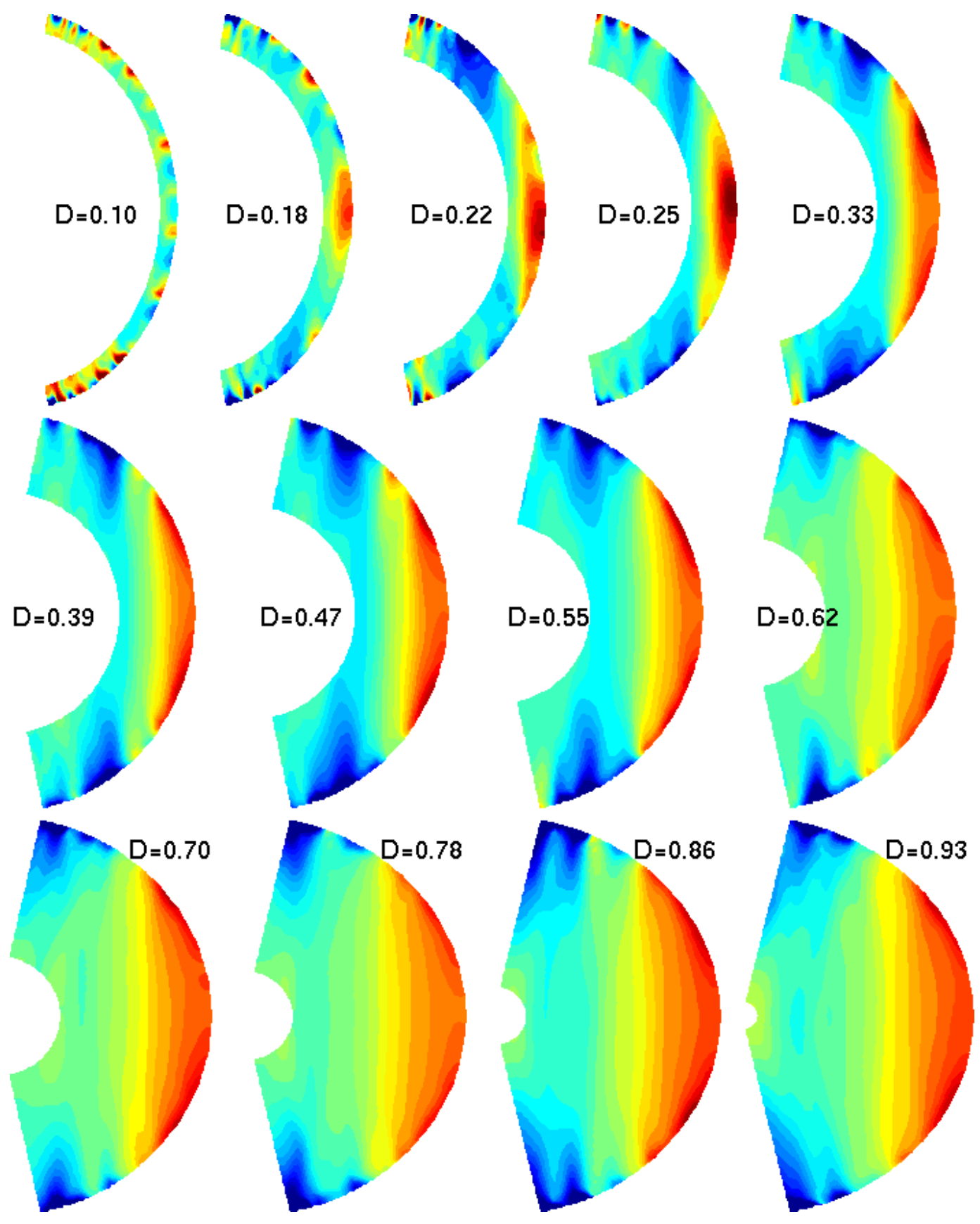

Figure 6.1: The zonal mean zonal velocity as function of model depth. $D \equiv \frac{r_{t}-r_{b}}{r_{t}}$ is the total depth where $r_{t}$ is the top boundary and $r_{b}$ is the bottom boundary. Red colors are eastward velocities and blue colors are westward velocity. The magnitude of the eastward velocity can be seen in Figure 6.3. 


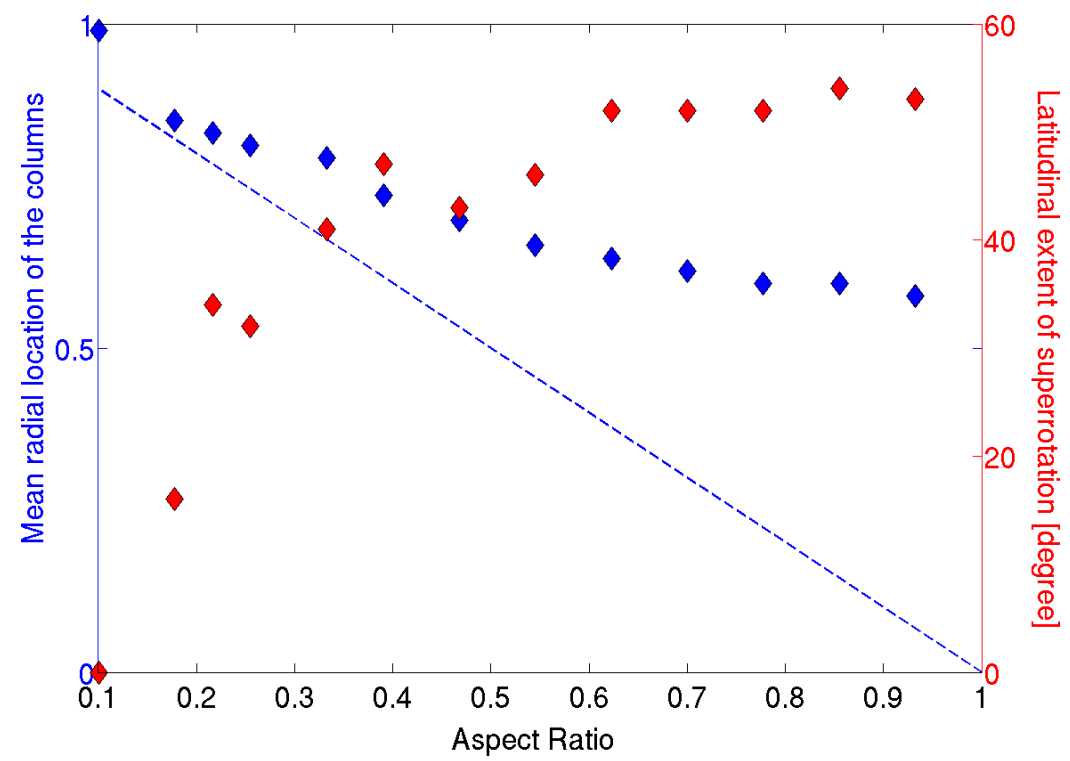

Figure 6.2: Location of columns (blue) in terms of the fraction of the radius covered by the model as function of model depth. The dashed blue line is the total depth of the fluid. The red dots are the meridional extent of equatorial superrotation as function of model depth. Each point is a numerical experiment ran to a statistically steady state.

thinnest case $(D=0.1)$ we do not find superrotation, perhaps because there wasn't enough resolution for formation of columns; or since $\chi$ does depend on the depth of the domain, and decreases with depth, then this thinner case may be in a parameter regime where rotation is not dominant $\chi<1$ similar to the case of slow rotation. As can be understood from our analysis in chapter 5 the smaller latitudinal extent is due to the columns being closer to the outside due to the smaller overall depth. However as the model becomes deeper the columns develop further from the bottom boundary. This shows that for a shallow model the choice of the location of the bottom boundary sets the width of the superrotating jets, and perhaps the depth of the dynamically significant region can be therefore deduced from the observations of the jets in the outer atmosphere. Calculating this depth based on the observations of Jupiter and Saturn gives a bottom boundary at approximately 0.07 and 0.2 respectively. Previous numerical convection models have chosen a shallower domain than the one used in previous chapters and indeed had a narrower superrotating jet. Note that even if the dynamics are confined to a relatively shallow domain, it still will contain most of variation in density and pressure seen in the deeper model we have been using, and it 


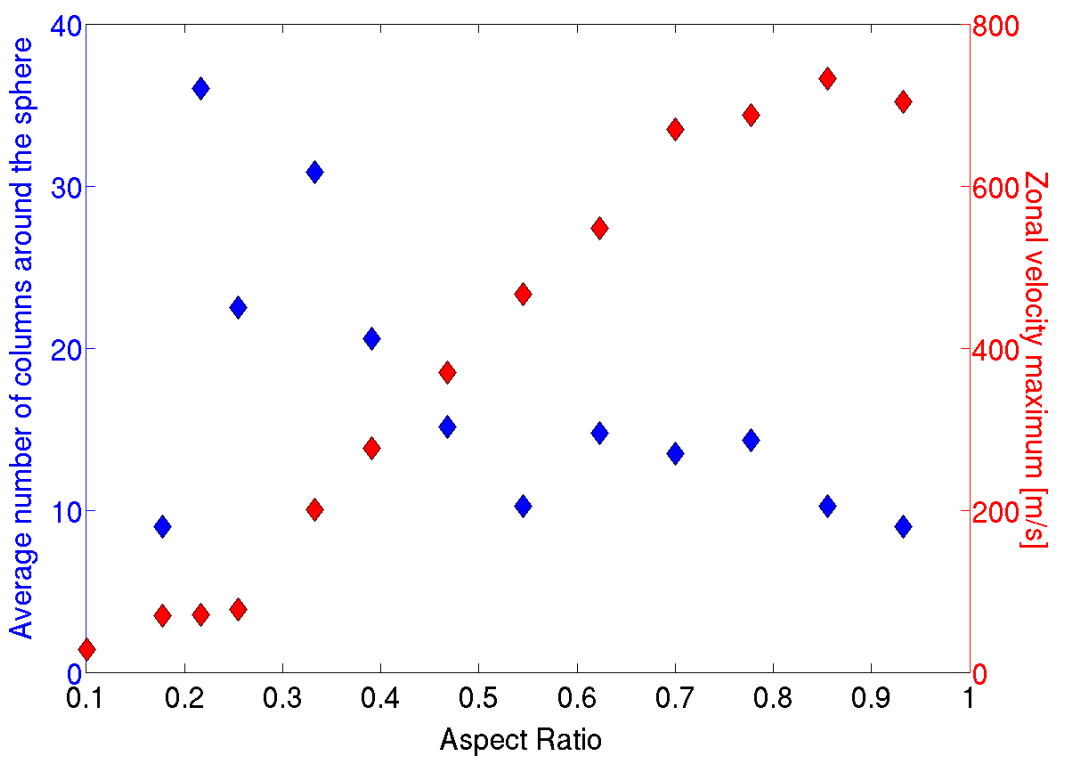

Figure 6.3: Number of columns (blue) and zonal velocity intensity (red) as function of model depth.

would have been harder to identify the mechanisms driving these dynamics working with only the shallower domain.

An interesting feature is that as the model gets deeper, approaching a full sphere, the columns do not move significantly deeper and the resulting width of the superrotation does not extend much beyond $50^{\circ}$ in latitude. This is shown more explicitly in Figure 6.2 which shows on the left (blue) grid the location of the columns (taken as the averaged radial location of the maximum in equatorial $2 \mathrm{D}$ streamfunction) as function of the aspect ratio $(D)$. Each point represents a numerical experiment, and the dashed line is the total depth of the fluid. As the aspect ratio grows (model gets deeper) the location of the column drifts slowly inward but becomes further away from the bottom boundary. Looking at the equatorial plane we can identify the columns and similarly to the standard case shown in chapter 2 they are all cyclonic.

The number of columns (estimated by a Fourier analysis of the 2D streamfunction on the equatorial plane) around the $360^{\circ}$ equatorial plane is higher for smaller aspect ratios and is fairly constant as the model becomes deep. This is shown in Figure 6.3. However the intensity of the columns and the resulting superrotation grow with depth even though forcing is constant. This is despite $\chi$ becoming bigger as the model is deeper. 

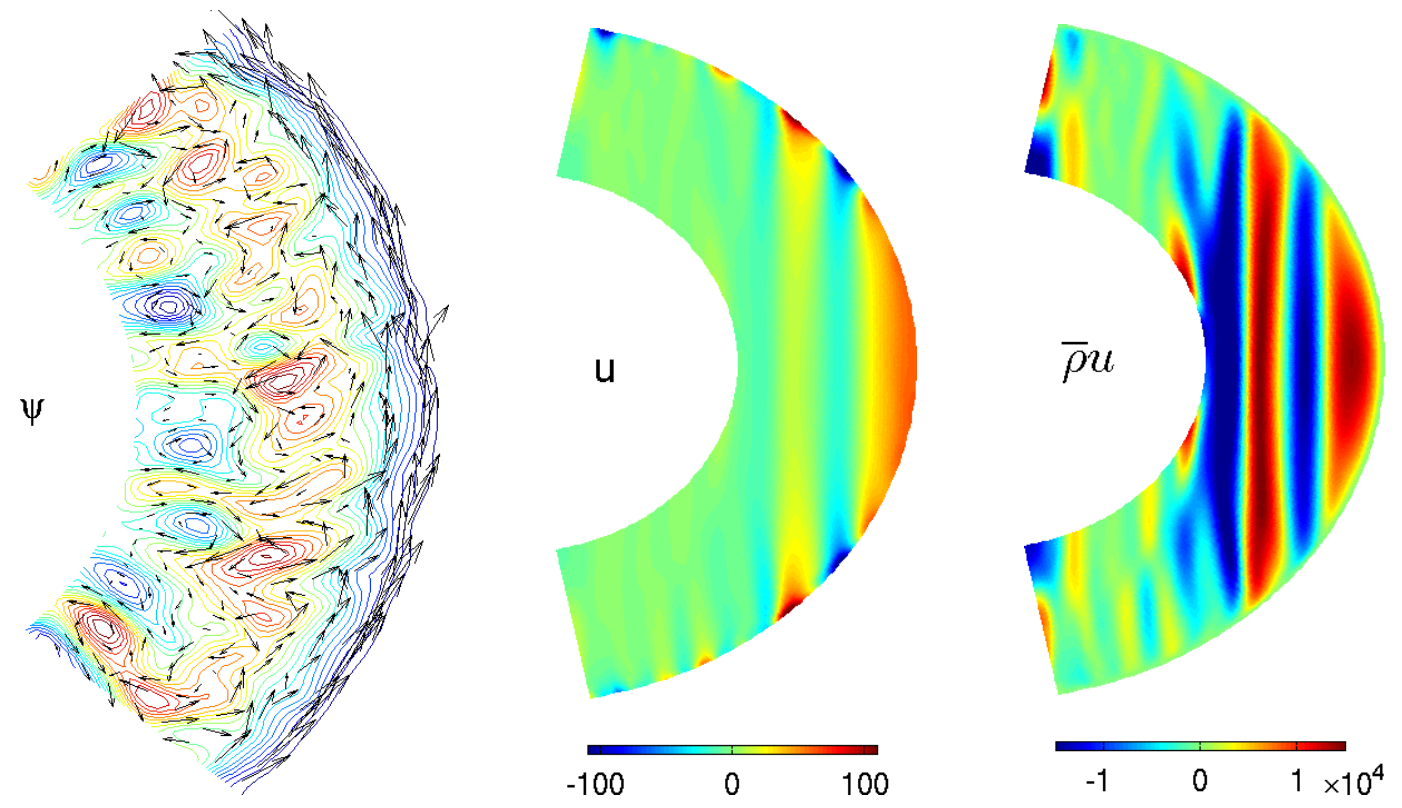

Figure 6.4: A solution with multiple columns. This structure appears during spin-up but in time will reduce to having only cyclonic columns. left: the equatorial streamfunction containing both cyclones and anticyclones. middle: the total momentum $\bar{\rho} u$ in meridional section; right: The zonal velocity in a meridional section. Meridional fields are of snapshots taken at 17 days from spin up and the equatorial slice is at 25 days.

\subsection{Multiple Column Layers}

In Busse's original heuristic picture (Busse, 1976) for multiple zonal jets driven by interaction between cylinders, he suggested that multiple columns at different radii from the center may interact to cause alternating jets. In this picture the cylinders were confined to the region outside the tangent cylinder surrounding the core and extended throughout the planet. The discovery of jets at high latitude (Porco et al., 2003) later overshadowed this suggestion since this would require the layers of columns to extend deep into the region contained within this tangent cylinder. Only if the internal region with no dynamics would be very small could such a scenario be plausible. None of the linear models, or the numerical Boussinesq models have found solutions with multiple column layers. In the previous section we have shown that even when extending the model almost all the way to a full sphere we find only one layer of columns and they are located at an equal distance from the axis of rotation.

In this section we show that we often get such multiple columns during the spin- 
up, however eventually due to shear they disappear and we find the solutions with one dominant layer of column at an equal distance from the axis of rotation. Figure 6.4 shows on the left an equatorial slice where we find an inner set of anticyclones (blue) and at a greater radius a set of cyclones (red). Looking at different slices shows that, similar to the case of Figure 4.13, these features extend as columns, parallel to the axis of rotation, to the outer levels. The middle panel shows the zonal velocity with multiple east-west zonal jets at the surface. Such a picture would be desirable

for the Jupiter case with a wide superrotating jet and then alternating jets at high latitudes, however we find that such a scenario is not stable and the multiple columns eventually disappear. It is interesting that at this stage $\bar{\rho} u$ is nearly constant along the axis of rotation, meaning as discussed in section 4.3.2 that the baroclinic vorticity production has a near equal and opposite contribution from entropy and pressure fluctuations. In time the interior part of the column becomes more barotropic and the solution looks like Figure 3.9. The parameters for the run presented here are the same as the in Figure 3.9, and are of instantaneous fields. We find that as we decrease the viscosity such solutions survive for larger times, despite the increase in magnitude, or the circulation and vertical shear. It is possible that therefore that experiments with higher resolution, where we can use smaller viscosities would have stable solutions with such multiple columns and surface alternating zonal flows.

\subsection{Model Sensitivity to Nondimensional Parame- ters}

In this section we look at the model sensitivity to Rayleigh, Prandtl and Ekman numbers. Due to the simplicity of the model, and the use of uniform viscosity and diffusivity coefficients the model is controlled by only these three parameters. Although these nondimensional parameters define the system, since the GCM is not naturally written in terms of these parameters, we find it useful to study both the effect of the nondimensional parameters and the physical parameters that compose them, namely the viscosity, diffusivity and heat flux. There is an obvious overlap between these two approaches, but as we show they are not redundant and it is helpful in looking at different slices through the parameter space. For most examples we keep the rotation period constant (9.92 hours), and therefore Ekman number (and 


\begin{tabular}{|c|c|c|c|c|c|c|c|}
\hline experiment & $R a$ & $E k$ & $\operatorname{Pr}$ & $\chi^{-1}=R a \cdot \operatorname{Pr}^{-1} E k^{2}$ & varying param. & range & color \\
\hline$R a 1$ & varies & $1.5 E-4$ & 10 & varies & $R a$ & $1 E 6-5 E 7$ & red \\
\hline$R a 2$ & varies & $4 E-4$ & 10 & varies & $R a$ & $5 E 5-2 E 7$ & blue \\
\hline$R a 3$ & varies & $8 E-4$ & 10 & varies & $R a$ & $1 E 5-1 E 7$ & black \\
\hline $\operatorname{Pr} 1$ & $3 E 6$ & $4 E-4$ & varies & varies & $\operatorname{Pr}$ & $0.8-12$ & green \\
\hline $\operatorname{Pr} 2$ & varies & $4 E-4$ & varies & 0.048 & $\operatorname{Pr}$ & $0.1-5 E 3$ & purple \\
\hline$E k 1$ & $3 E 6$ & varies & 10 & varies & $E k$ & $1.5 E-4-1.5 E-3$ & magenta \\
\hline$E k 2$ & varies & varies & 10 & 0.048 & $E k$ & $1 E-4-1.5 E-3$ & gray \\
\hline$E k 3$ & $3 E 6$ & varies & 10 & varies & $E k$ & $7 E-5-7 E-3$ & orange \\
\hline
\end{tabular}

Table 6.1: Table of parameters for numerical experiments in chapter 6 .

alternatively Taylor number) will depend only on the viscosity. The results will be presented in terms of Ekman numbers and not the Taylor numbers but can be easily converted. For convenience we write again the nondimensional numbers

$$
\operatorname{Pr}=\frac{\nu}{\kappa}, \quad T a=\frac{4 \Omega^{2} H^{4}}{\nu^{2}} \quad R a=\frac{B_{0} H^{4}}{\nu \kappa} \quad E k=\frac{\nu}{2 \Omega H^{2}}
$$

where $H$ is the total vertical extent of the model, and $B_{0}$ is given by

$$
B_{0}=\frac{Q}{\bar{s} \bar{T}_{0}}\left(\frac{g_{0}}{H}\right)^{\frac{1}{2}}
$$

where the subscript 0 denotes the top level. We try and explore a parameter regime as wide as the configuration and computational resources allow us. Ideally we would like to increase Rayleigh numbers by decreasing the viscosity and diffusivity while keeping reasonable heat fluxes, and therefore make the model as turbulent as we could. This however is limited by the grid scale. For example the standard $1^{\circ}$ resolution configuration has $360 \times 160 \times 120 \times 6 \simeq 4 E 7$ computations per time step. Time steps are small (typically 5 seconds) due to the convective nature of the system, and therefore calculations are computationally demanding. We find it therefore useful for these series of numerical experiments to use the low resolution $2^{\circ}$ runs. These runs are presented in Table 6.1 where only $R a 1$ are $1^{\circ}$ resolution cases. An important component of this analysis is the effect of the rotation period. This has been discussed in both sections 3.1 and 4.5, and therefore we will refer to those sections for this discussion. We will use these results, though, in our discussion on the effect of the Ekman number. 


\subsubsection{The Rayleigh Number}

We begin with experiments where we vary the Rayleigh number while holding the Prandtl and Ekman numbers constant. The Rayleigh number depends on all three physical parameters (viscosity, diffusivity, and heat flux), and therefore since rotation period (9.92 hours) is held constant, then a constant Ekman number implies a constant viscosity. Then in effect in this set of experiments only the amplitude of the heat flux is varied. We repeat these experiments $(R a 1-3$ see table 6.1$)$ for different values of Ekman numbers denoted in Figure 6.5 with different colors. Two degree resolution runs are denoted by diamonds, while one degree resolution runs are denoted by squares. Each numerical experiment presented here, denoted by a single dot, has been run to statistical steady state and data has been taken from the instantaneous fields.

The left panel in Figure 6.5 shows that as the Rayleigh number increases the magnitude of the equatorial superrotation increases as well. A stronger heat flux provides more potential energy (from the convection) to the system resulting in a stronger kinetic energy and superrotation. For the more energetic runs, the curves level off reaching possibly an asymptotic limit. Christensen (2002) suggested a linear relationship when looking at the Rossby number as a function of the heat flux. Runs of similar Rayleigh number will have higher velocities for higher Ekman number, though the model resolution seems to possibly have an impact as well. This shows that the magnitude of the superrotation in our runs does depend on the magnitude of the forcing, however this dependence may decrease for high Rayleigh numbers as indicated particularly by the higher resolution runs (red). In section 4.7 we have shown that although this magnitude does depend on the choice of the Rayleigh number it is still consistent with mixing length theory estimates for the magnitude of the velocity as function of the forcing. For the whole range of Rayleigh numbers that we have experimented with we have found that the convective structures and mechanisms studied in chapter 5 are consistent. In Boussinesq, Cartesian, Rayleigh-Benard convection experiments Sprague et al. (2006) have found that as Rayleigh number is increased the flow within the columns increases in strength, as in our experiments, but leading eventually to a breakdown of the Taylor columns due to enhanced lateral mixing. In our experiments we have not found this to happen, and actually have found the ratio of the vorticity of the columns to the background vorticity to grow with Rayleigh number, with more profound columns. However, even if the columnar 

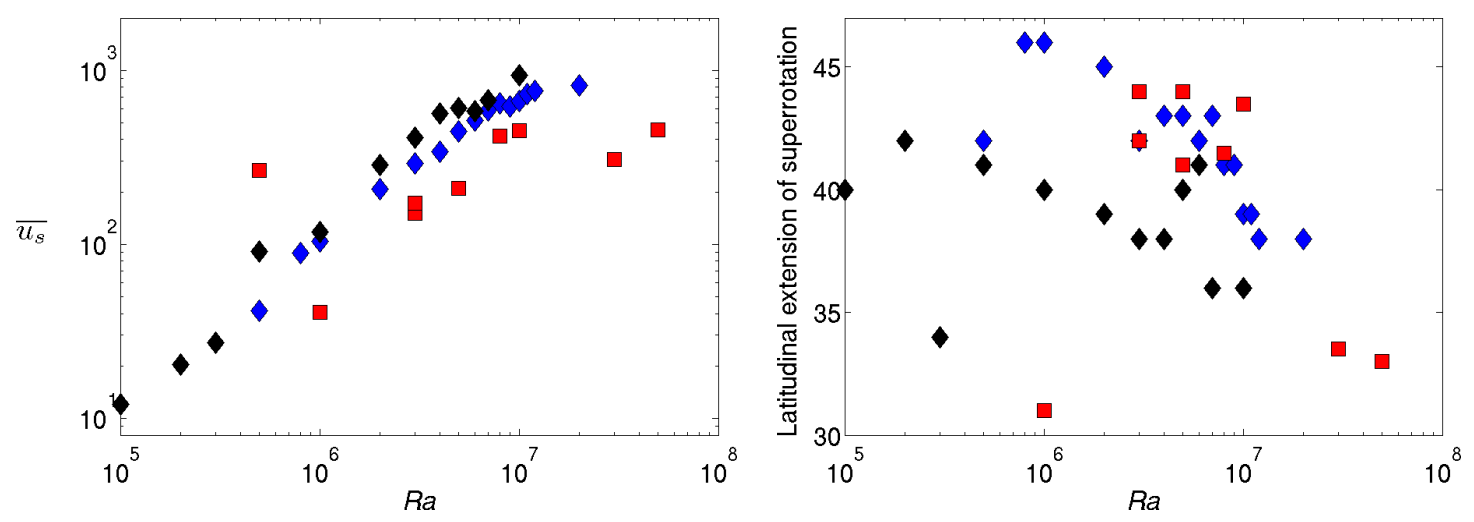

Figure 6.5: The model sensitivity to Rayleigh number. left: The magnitude of equatorial superrotating zonal velocity $[\mathrm{m} / \mathrm{s}]$; right: latitudinal extent of equatorial superrotation [degree];

structure would break for some higher Rayleigh number, the convective plumes will still be aligned with the rotation axis (as in the Sprague et al. (2006) experiments at high Rayleigh number), and therefore much of the angular momentum flux and the mechanisms described in chapter 5 will still hold.

The equatorial superrotation on Jupiter extends roughly to latitude $17^{\circ}$ and on Saturn to latitude $30^{\circ}$ (Figure 1.1). We have shown in section 6.1 that in our model the latitudinal extent can depend on the depth of the domain for shallow cases (Figures $6.1,6.2$ ). In Figure 6.5 we show that this latitudinal extent depends on the Rayleigh number as well and runs with higher heat flux develop a narrower equatorial superrotating jet.

Extrapolating these results to the regimes relevant to Jupiter and Saturn is difficult since the model (eddy) viscosities are many orders of magnitude larger than mean molecular viscosities. Therefore to maintain a large Rayleigh number we must compensate with a larger heat flux. If one used the Rayleigh number with the eddy viscosities to calculate the actual heat flux, the resulting flux would be many orders of magnitude too large.

A useful measure which eliminates the dependence on molecular parameters will be $\chi^{-1} \equiv R a \cdot \operatorname{Pr}^{-1} E k^{2}$. We have shown already (section 3.1.1) that this parameter determines the level to which the convective plumes are aligned with the rotation axis, separating therefore between the rotationally dominated convection to gravitationally dominated convection. Using the parameter $\chi^{-1}$ allows comparing a larger set of numerical experiments. For most cases (all but Ek3) the rotation period is constant 
and therefore $\chi^{-1}$ is a measure of the magnitude of the convection. In the upper panels of Figure 6.6 we look again at the magnitude and latitudinal extent of the superrotation. The upper bound of $\chi^{-1}$ values is the limit where the convection will not be aligned with the rotation axis. At that point no convection columns will appear and the whole mechanism for superrotation described in chapter 5 will break. As seen in Figure 4.11 at this point the equatorial flow would rapidly switch from super-to-sub rotating. The lower limit of $\chi^{-1}$ is when convection is weak and is limited by either the critical value of convection (3.11) or numerical limitation of the eddy Ekman number. It can be seen on the left panel that the higher resolution experiments can reach lower $\chi^{-1}$ values since the higher resolution allows having a smaller eddy viscosity. The importance of this is that they are supercritical for lower $\chi^{-1}$ numbers, and seem to reach a point where more turbulent runs do not necessarily have stronger equatorial velocities. This is seen also but to a lesser extent in the $2^{\circ}$ resolution runs. Such a scenario will mean that the velocities in our model, which are on the order of magnitude of the winds on Jupiter and Saturn, might be more robust than indicated by the slope in Figure 6.5.

In the lower left panel in Figure 6.6 shows the dominant wavenumber for the streamfunction on the equatorial plane. This will serve as an approximation for the number of columns surrounding the interior core. The results indicate that the further the model is into the rotationally dominated regime, the more convection columns we find around the equatorial plane. As a caveat, note that since there is a clear separation between the high and low resolution results the numerical values are affected by the model resolution. These results might imply that going to higher resolution runs with lower Ekman numbers will lead to significantly more convection columnar structures which will result in a higher frequency waves on the surface. The waves observed on the surface of Jupiter (Reuter et al., 2007) have a $300 \mathrm{~km}$ wavelength which is currently $\sim \frac{1}{3}$ of the resolution of our runs. Therefore it is hard to identify our results with the observed waves; however the spatial resemblance (crests that are curved eastward and centered at the equator), the phase speed which is about equal to the mean zonal velocity (as in the model), and this result suggesting that the number of columns (and therefore resulting waves), will increase with resolution brings us to hypothesize that the observed waves might be a surface manifestation of finer structure convection columns.

The lower right panel in Figure 6.6 compares the full kinetic energy to that of the 

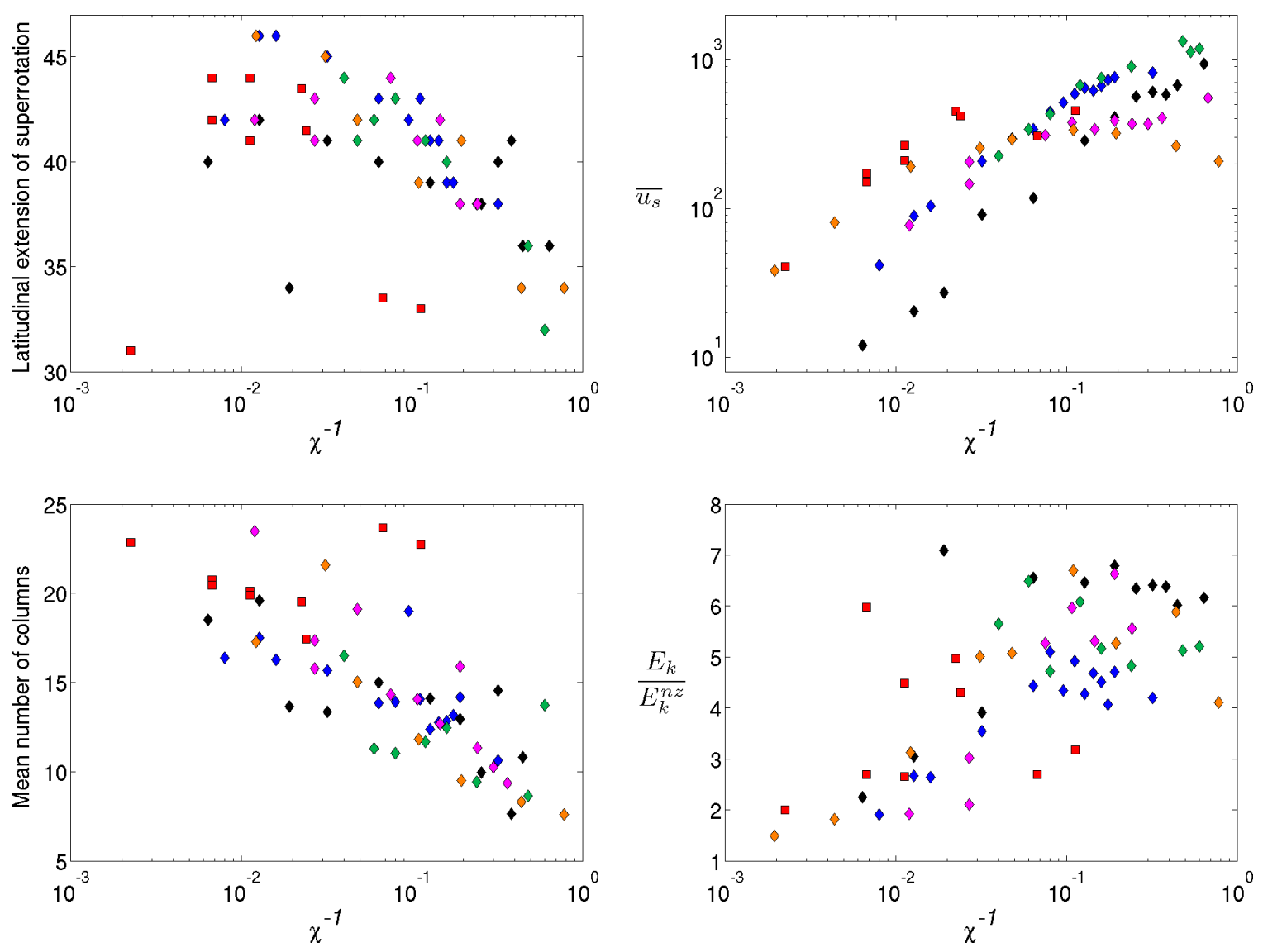

Figure 6.6: Model dependence on $\chi=R a \cdot \operatorname{Pr}^{-1} E k^{2}$. Run color code corresponds to parameters in table 6.1. upper left: latitudinal extent of equatorial superrotation [degree]; upper right: magnitude of equatorial superrotating zonal velocity $[\mathrm{m} / \mathrm{s}]$; lower left: mean number of columns around the equatorial plane; lower right: ratio of total kinetic energy to the kinetic energy of the non-zonal components.

non zonal components. In all cases we find that the zonal kinetic energy dominates. Although it is hard to follow a particular trend for a specific set of runs, in general it seems that the higher energy runs have a stronger zonal component in the total kinetic energy. Some of the sets of runs reach a maximum beyond a specific heat flux but determining this will require more runs.

We conclude that in the parameter regime we have studied the Rayleigh number does affect both the amplitude and the latitudinal extent of the jet. We may expect that runs at higher resolution which will be capable of higher Rayleigh numbers and lower Ekman numbers will not depend (magnitude wise) on the Rayleigh number and will have (even without the effect of the bottom boundaries) a narrower superrotation. 

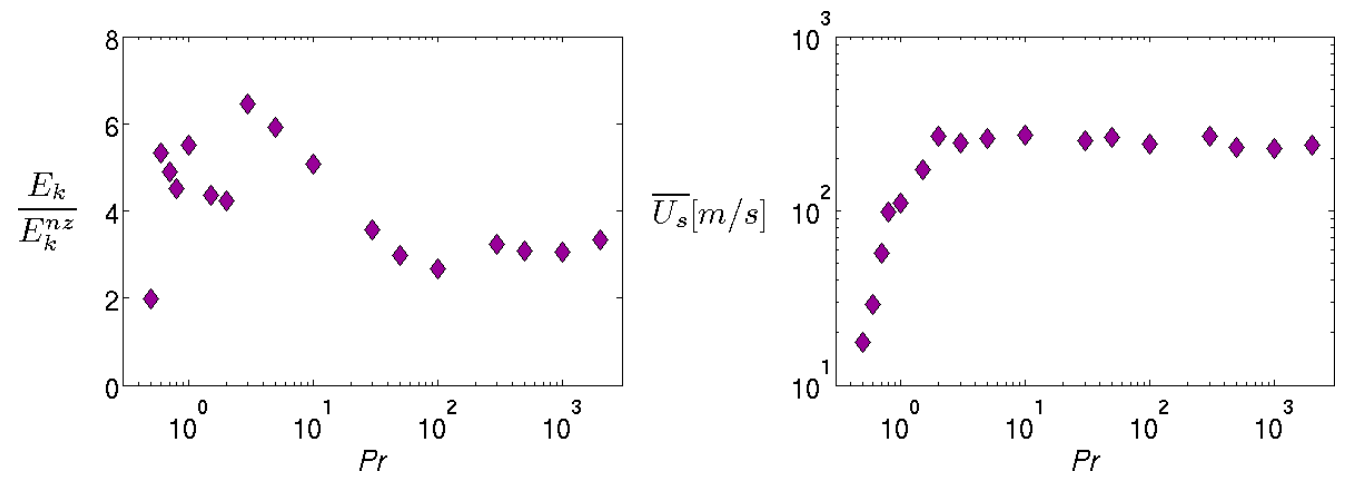

Figure 6.7: The dependence on Prandtl number with constant heat flux (diffusivity). left: ratio of total kinetic energy to the kinetic energy of the non-zonal components. right: magnitude of equatorial superrotating zonal velocity $[\mathrm{m} / \mathrm{s}]$.

\subsubsection{The Prandtl Number}

To study the dependence on the Prandtl number we perform two sets of experiments. In one we hold the Ekman and Rayleigh numbers constant and vary the Prandtl number $(\operatorname{Pr} 1)$. In this case diffusivity varies and the heat flux adjusts accordingly to keep the Rayleigh number constant. These results have been presented in Figure 6.6 and have a similar effect to holding the Prandtl number constant and varying the heat flux. The second set of experiments (Pr2) is holding the Ekman number constant and varying the Prandtl number while the heat flux is constant $\left(\chi^{-1}=0.048\right)$, so that the Rayleigh number will vary as well. In this case, since only diffusivity varies $R a \cdot \mathrm{Pr}^{-1}$ is constant. In Figure 6.7 we look at the results for this experiment. The right side plot shows on the horizontal axis both the Prandtl number (bottom) and Rayleigh number (top) since only diffusivity is varying. It shows the increase in the mean amplitude of the superrotation up to a level where beyond it the mean velocity remains roughly constant. This plot can be seen as an extension of the corresponding plot in Figure 6.5 extending into a region of higher Rayleigh number so that the magnitude of the velocity is no longer a function of the Rayleigh number. Despite the high Rayleigh numbers the run is not more turbulent and only means that beyond $\operatorname{Pr} \sim 2$, for these run parameters, diffusivity is small so that the amplitude of the zonal velocity is insensitive to the Prandtl number.

We find that the latitudinal extent of the superrotation does not depend on the diffusivity. Runs with higher Prandtl number do have a higher wavenumber to the equatorial streamfunction on the equatorial plane, and as in the case of the zonal 

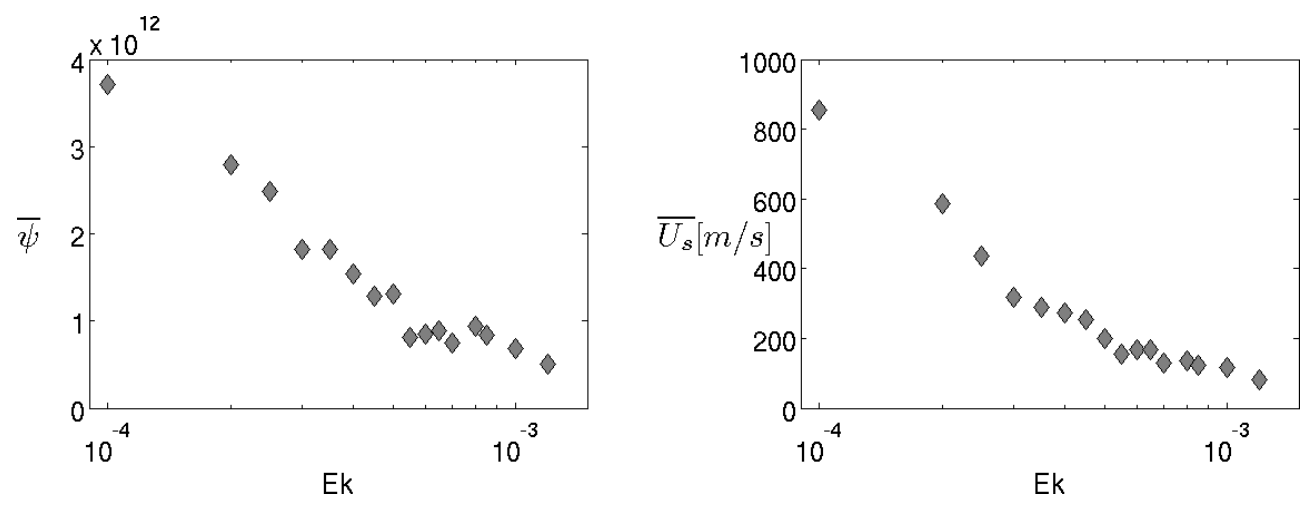

Figure 6.8: The model sensitivity to viscosity. left: maximum values of equatorial streamfunction. right: surface superrotation zonal velocity.

velocity it becomes constant beyond a certain point for high Prandtl numbers. The left panel in Figure 6.7 shows the ratio of full kinetic energy to that of the non zonal components and indicates higher ratios for lower Prandtl numbers. The run with $\operatorname{Pr}=0.5$ is the weakly nonlinear run presented in section 5.1.

\subsubsection{The Ekman Number}

We study the effect of the Ekman number in three sets of experiments. In the first we keep the Rayleigh and Prandtl numbers constant and vary only the Ekman number (keeping again the rotation constant) so that we change effectively the viscosity and the heat flux adjusts accordingly. In the second set of experiments we keep the Prandtl number and heat flux constant, so that when varying the Ekman number (viscosity), the Rayleigh number changes as well. The third experiment is similar to the second one only varying the Ekman number by changing the rotation period instead of the viscosity.

Beginning with the first case, since the Prandtl number and heat flux are constant we look at the model effectively as only the viscosity changes. In Figure 6.8 we show the magnitude of the equatorial streamfunction and superrotating zonal velocity as functions of the Ekman number. We find, as can be expected, that as viscosity increases the magnitudes of both decreases. Going back to the zonal momentum balance in (4.20), as angular momentum is fluxed outward by the eddy fluxes, the balance between the eddy flux term and the viscosity happens earlier as $\nu$ increases, and therefore both the superrotating winds and the rotation of the columns (correlated 

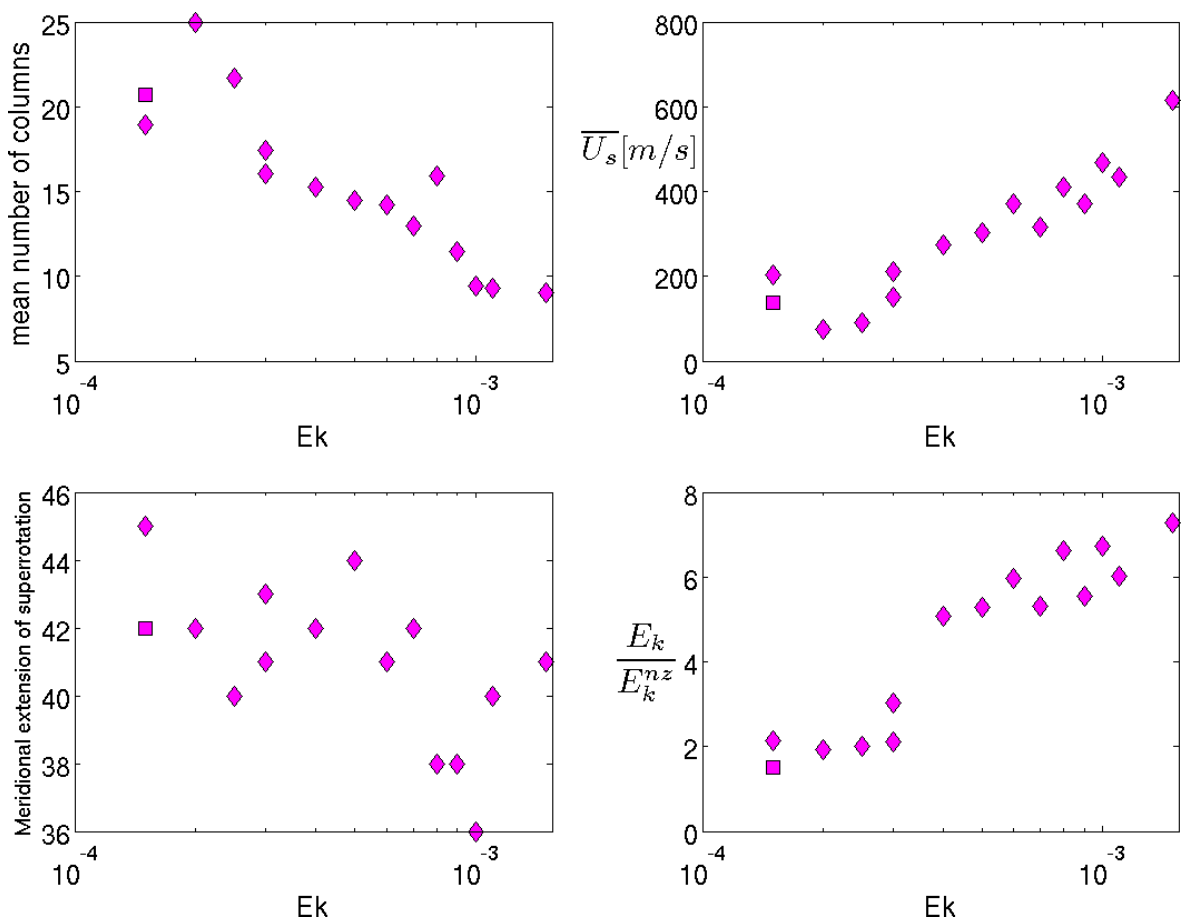

Figure 6.9: The model sensitivity to the Ekman number. upper left: The mean number of columns (wave number) on the equatorial plane; upper right: mean surface zonal velocity of the superrotation; lower left: Meridional extension (latitude) of the superrotation; lower right: ratio of full kinetic energy to the non zonal kinetic energy.

to the eddy angular momentum flux) are weaker.

For the second case keeping both Prandtl and Rayleigh numbers constant as the Ekman number (viscosity) varies, the heat flux adjusts accordingly. Therefore a higher Ekman number means a larger heat flux, and thus we find that the strength of the superrotation increases with Ekman number, although Rayleigh number is constant. This means that the strength of the superrotation is related to the heat flux and not to the values of the Rayleigh number itself. On the other hand, as we increase the Ekman number the number of columns, which is estimated by a Fourier analysis of the streamfunction, on the equatorial plane decreases. This indicates that in a higher resolution model where we would be able to reach lower Ekman numbers we may expect to find more columns and surface waves. In addition as we increase the Ekman number the meridional extent decreases although it levels off for lower Ekman numbers. Finally the ratio of the full kinetic energy to that of the non zonal components grows with Ekman number. 


\subsection{The Effect of the Forcing Profile}

In section 2.5 we discussed the use of an adiabatic vertically continuous forcing profile, which does not confine the convection to the boundaries and is a way of representing the longer time scale cooling of the planet. Other models (e.g. Heimpel and Aurnou 2007) have used isothermal boundaries and therefore forced Rayleigh-Benard type convection; however this is an extreme oversimplification of the forcing and the planet does not have an isothermal boundary. A similar boundary dependent forcing
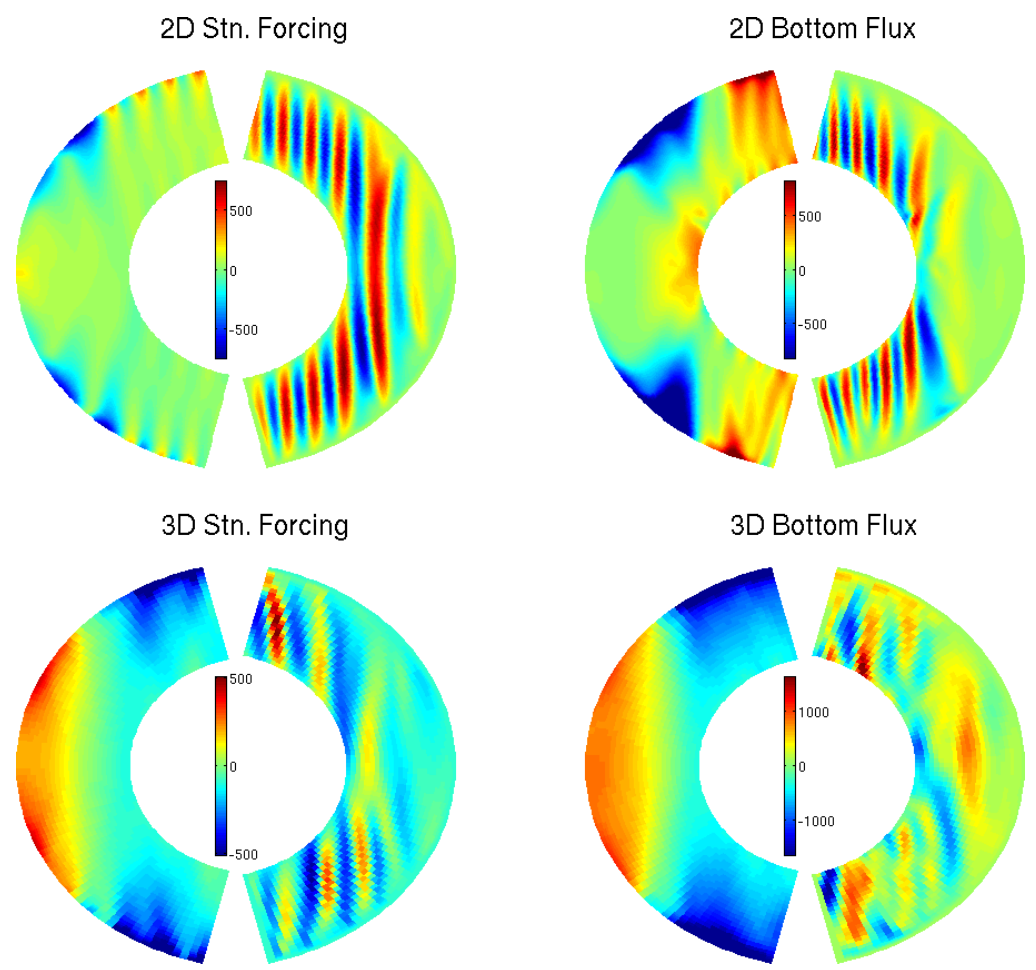

Figure 6.10: Comparing different forcing profiles for 2D and 3D cases. upper left: $2 \mathrm{D}$ with vertical forcing profile (section 2.5). upper right: $2 \mathrm{D}$ with bottom flux and Newtonian cooling on top; bottom left: 3D with vertical forcing profile; bottom right: $3 \mathrm{D}$ with bottom flux and Newtonian cooling on top.

would be applying a heat flux at the bottom boundary and relaxing to a reference temperature on top. We have used this profile for the discussion about the critical Rayleigh number in section 3.1.2. Although less realistic than the continuous profile it is worth comparing the statistically steady state solutions to learn if the result is dependent on the form of the forcing. Here, we compare the results using both types of forcing for both the 2D and 3D cases. As can be seen in Figure 6.10 we find that 
for the 2D experiments a bottom heat flux will cause the dynamics to be constrained to the inner part of the sphere. For the 3D although at the initial stages (not shown) the convection is different (plumes rising from the bottom boundary), after enough time the profiles with both types of forcing become quite similar. The reason this makes a difference in the $2 \mathrm{D}$ case is the constraint given by (3.13) which is even more limiting in this case because of the convective plumes only rising from below. Note that the $3 \mathrm{D}$ runs are of $2^{\circ}$ resolution; as discussed in section 6.3 the higher latitudes do not maintain the finer structure seen in the $1^{\circ}$ resolution runs such as in Figure 3.9. All cases here are using the full anelastic density variation.

\subsection{Summary}

The deep anelastic GCM we developed and studied in previous chapters is analyzed over a range of parameters. Such a study is essential in order to get a feel for the parameters of the model. Due to the simplicity of this idealized GCM the parameter regime is limited to mainly three nondimensional parameters beyond the geometric configuration of the model and the choice of forcing profile. We perform sets of numerical experiments changing both the geometric configuration of the model and the nondimensional control parameters. We find that using a shallower or deeper domain preserves to the most part, the main characteristics of the circulation, including the superrotation and convection columns. A shallower domain which is consistent with some of the recent MHD estimates (Liu, 2006), would in fact limit the superrotation to a narrower latitudinal band which is consistent with the observations on Jupiter and Saturn. On the other hand extending the model to a full sphere does not extend the superrotation to the poles, and beyond a certain depth the superrotation is close to being invariant to the depth of the domain.

We find that the magnitude of the superrotation in our model does depend on the Rayleigh number for the parameter regime studied. An important distinction is whether the amplitude of the superrotation is sensitive to the full Rayleigh number or to the heat flux itself. The results at this point are still indecisive. If the total Rayleigh number is key, then the use of large eddy viscosities is justifiably compensated by the use of large heat fluxes. If on the other hand, the effect of the viscosity saturates at some limit (still far from the molecular limit) then possibly the velocities resulting from realistic heat fluxes will be considerably smaller. For the parameter regime 
we have been able to explore this questions remains open. We find that beyond a certain limit the Prandtl number becomes irrelevant due to the small diffusivities, and although Rayleigh numbers will grow for such a case, effectively the circulation will not change. In general we find that using smaller Ekman numbers and larger Rayleigh numbers (which are in the direction of more realistic numbers), will result in more, and smaller scale, convection rolls and a resulting superrotation which is more latitudinally confined.

Other possible solutions, such as ones with multiple layers of convection columns, which currently naturally appear during spin-up, but usually are sheared apart as the model spins-up toward having one layer of cyclonic convection columns, have been shown. Since for the lower Ekman number cases these solutions are sustained for a longer period, despite the shear being large, we suggest that in high resolution runs we may find such solutions which are stable. 


\section{Chapter 7}

\section{Formation of Multiple Zonal Jets by Baroclinic Instability}

\subsection{Introduction}

In this chapter we use a simplified model to look at a different aspect of the dynamics. The full GCM has been instrumental in understanding the mechanism for superrotation and the dynamics arising from convection in a rotating spherical deep system. We have seen the formation of columnar modes which propagate eastward due to the background planetary vorticity gradient. The mechanism causing the propagation of these modes is similar to that of a standard Rossby wave on the exterior of a sphere, only that the planetary vorticity gradient is in the opposite direction, thus growing as one goes to lower latitudes (the equivalent of moving radially outward in the interior of a sphere).

The opposite planetary vorticity gradient can be thought about in terms of conservation of vorticity in column of fluid. If constrained to a thin spherical shell then as as a fluid column moves towards the axis of rotation the fluid column shrinks in length, and therefore this would be equivalent to a sloping surface with a positive slope, as the planetary vorticity grows, and therefore a positive $\beta$ effect. On the other hand columns which penetrate the depth of the planet as we have seen in our GCM (Figure 4.13 ), will stretch as they move towards the axis of rotation, and therefore will be equivalent to having an opposite sloping surface to conserve planetary vorticity which is equivalent to a negative $\beta$ effect. Following the approach of Ingersoll and Pollard 
(1982) we have shown that for an anelastic fluid the radially varying density profile will cause a larger magnitude negative $\beta$. The equivalent $\beta$ for both the Boussinesq and anelastic cases can be seen in Figure 5.9.

We have discussed the two very different and essentially decoupled approaches used to model the atmospheres of the giant planets (section 1.3). We have shown that the deep approach which we have taken for our convection model can explain some elements of the dynamics such as superrotation, meridional poleward heat transport and possibly some of the waves observed within the equatorial superrotation. However, elements such as the formation of multiple zonal jets do not appear in the deep anelastic model. Our simulations indicate that possibly a higher resolution model with smaller viscosities will be able to produce more meridional structure in the zonal wind field. Examples of such solutions we present in section 6.2. Nevertheless, the similarity of the observations to weather patterns seen on Earth, and the existence of a thin but important stably stratified layer at the top of the atmosphere due to solar insulation, leads us to assume that there are important components to the dynamics beyond the convectively driven system.

Therefore in this chapter we look at a simplified model which contains components from both the shallow and the deep approaches. We use a two layer quasigeostrophic model where the upper layer is a standard quasigeostrophic layer on a $\beta$ plane, and the lower layer represents the deep interior convective columnar structure using a negative $\beta$ plane. The model is shallow in the sense that is quasigeostrophic and the jets are created by interactions of the eddies on a $\beta$ plane. However, the presence of the negative $\beta$ for the bottom layer makes the dynamics, and particularly the criterion for baroclinic instability, quite different than a standard quasigeostrophic $\beta$ plane model. We suggest that the interaction between the isentropic interior and the "weather layer" drives the multiple zonal jets.

This approach can be distinguished from previous shallow type models in several aspects. First, due to the weak meridional temperature in the upper atmosphere of the giant planets, baroclinic instability has been assumed to play a minor role in the dynamics of the jets. However as we show, due to the different geometry in the interior, even a weak baroclinic shear can result in substantial zonal flows that are stronger than the eddy field, and moreover baroclinic instability introduces a strong meridional variability in the velocity field. The instability acts as an energy source for the eddies, and the nonlinear eddy-mean interactions act to stabilize the flow. 
Therefore unlike previous shallow water or quasigeostrophic models which use either random forcing or deal with decay of strong initial perturbations, leaving it unclear how such a state can be maintained, this model accounts for the energy of the eddies through baroclinic instability. Baroclinic instability provides an energy source, so that energy does not have to be pumped in to maintain the jets. Other baroclinic instability models (Panetta, 1993; Williams, 2003), require large-scale baroclinicity strong enough to satisfy the Charney-Stern theorem, but which may be larger than the level of baroclinicity on the giant planets.

Second, the observed winds violate the barotropic stability condition (Ingersoll and Cuong, 1981), thus $\beta-u_{y y}<0$ at some latitudes, although the zonal winds appear to be very stable. In contrast, all of the shallow models produce curvatures $u_{y y}$ which are smaller than $\beta$, so that the predicted jets are weaker or wider than the Jovian ones. We find that $\beta$ and $u_{y y}$ have similar values (thus the barotropic stability condition is violated) and still the jets are shown to be stable. Third, most previous model assume a boundary at a depth of about one scale height, with the fluid below being motionless. Although this model is not deep due to the quasigeostrophic assumptions we show that the jets in the upper levels are maintained and are baroclinic when the bottom layer gets deeper. Using the negative $\beta$ assumption gives some representation of the deep dynamics seen in the full convection model. Finally, in many cases (e.g. Panetta, 1993) the jets are obvious only in the zonally or time average profiles, while here the jets are seen in the instantaneous picture as well. A main difference is that in those models the scales of both the instability and the resulting jets are on the order of the Rossby deformation radius, while here there is a scale separation between the instability which is much smaller than the jets which are again on the scale of the deformation radius.

Several authors have used this idea of a negative $\beta$ plane. Ingersoll and Pollard (1982) developed a stability criterion for columnar motions inside of a compressible fluid sphere. Their equivalent barotropic stability equation has an effective $\beta$ which is negative and three times the value from the sphericity of the planet. On Jupiter and Saturn the observed winds are close to marginal stability according to this criterion. Yano and Flierl (1994) have used a negative bottom layer $\beta$ to demonstrate its effect on an isolated vortex like Jupiter's giant red spot in a zonal jet, and Yano (2005) suggested that this can effect the direction of the equatorial jet. We will show that having different and opposite-signed $\beta$ values in the shallow and deep layer makes the 
dynamics different and favorable for creating jets even for weak baroclinic shears.

We begin with analysis of the stability problem in a two layer quasi-geostrophic model similar to the Phillips model (Phillips, 1954), but with the lower layer deeper than the upper layer and having a different geometry represented by the different $\beta$. Unstable modes appear at high wave numbers for low shears, implying there may be a significant scale separation between the eddies and the mean flows generated by the nonlinear interactions and the energy cascade. Next, since the fastest growing mode is the key contributor to the initial instability, we develop an analytical theory for the nonlinear problem containing of only this mode and zonal flow corrections. This truncated model which is presented in section 7.4 gives an analytic expression for an induced zonal flow which has a multi-jet meridional structure, and which is not limited to the weakly supercritical case (Pedlosky, 1970) so that it can be as strong as the eddies. We show that this model can reduce to the weakly supercritical case in section 7.5.

Then, using a pseudo-spectral fully nonlinear numerical model containing many initial modes, we show that indeed an induced zonal flow with a multi-jet meridional structure is generated from the baroclinic instability. The truncated model predicts well both the number of jets and their amplitudes. This emphasizes the importance of the truncated model which allows us to isolate the physical mechanism of the jet formation before the system becomes turbulent. In time, as more unstable modes are generated, quasigeostrophic turbulence begins and an inverse energy cascade generates wider and stronger jets. Once the meridional scale of the jets has reached the Rhines scale, these jets become stable and in most cases have a bigger amplitude than the eddy field, thus creating a multi-jet structure across the whole channel. A complete description of the numerical experiments is given in section 7.6.

A few mechanisms govern the generation and stability of the zonal jets: baroclinic instability extracts energy from the basic shear at high wave numbers to form small scale eddies, eddy interaction creates an induced zonal flow with a strong meridional variation, and eddy-mean flow interaction creates exchange of energy between the eddies and the mean flow which stabilizes the flow. The truncated model allows us to isolate these phenomena. Baroclinic instability tends to sharpen and intensify the jet once it is created while quasi-geostrophic turbulence will tend to cascade the energy into larger scales. Both effects can be seen in the numerical experiments. A discussion of these mechanisms and its relation to the Jovian jets is given in section 7.7. 


\subsection{The Two-Beta Model}

We use a two layer quasigeostrophic model (Phillips, 1954), with a simple shear flow on a $\beta$ plane in a zonal channel of meridional width $L$. The layer thicknesses are different, such that the upper layer is much shallower than the lower layer, in order to represent a thin weather layer and a deep adiabatic interior. Although the two-layer model is often thought of as representing homogeneous incompressible fluids with the deep layer having a slightly larger density, Flierl (1992) argues that an isentropic interior with a thin weather layer of higher entropy gives the same equations. In order to parametrize the deep layer flows (Ingersoll and Pollard, 1982), we use a negative $\beta$ plane in the bottom layer and a standard $\beta$ plane for the upper layer, as discussed in section 7.1. The opposite-signed $\beta^{\prime} s$ make the stability problem quite different from the classical case (c.f. Pedlosky, 1970). There is a free interface between the two layers whose horizontal height gradient is related to the difference in pressure gradients within the layers. The quasigeostrophic inviscid potential vorticity equation for each layer, dimensioalized in the standard way as in Pedlosky (1987) is

$$
\begin{aligned}
{\left[\frac{\partial}{\partial t}+\frac{\partial \Psi_{n}}{\partial x} \frac{\partial}{\partial y}-\frac{\partial \Psi_{n}}{\partial y} \frac{\partial}{\partial x}\right]\left[\nabla^{2} \Psi_{n}+\right.} & \\
\left.(-1)^{n} F_{n}\left(\Psi_{1}-\Psi_{2}\right)+\beta_{n} y\right] & =0 .
\end{aligned}
$$

where $n$ denotes the layer, $\Psi_{n}$ is the stream-function and $F_{n}$ is the non-dimensional Froude number given by

$$
F_{n}=\frac{f^{2} L^{2}}{g^{\prime} D_{n}}
$$

where $f$ is the Coriolis parameter, $g^{\prime}$ is the reduced gravity, and $D_{n}$ is the layer depth. For future notation we denote the full potential vorticity in each layer as

$$
\Pi_{n}=\nabla^{2} \Psi_{n}+(-1)^{n} F_{n}\left(\Psi_{1}-\Psi_{2}\right)+\beta_{n} y
$$

We will assume the simplest basic state with a uniform flow in each layer,

$$
\Psi_{0 n}=-U_{n} y
$$


The total streamfunction is composed of the mean part (7.4) and a perturbation

$$
\Psi_{n}=\Psi_{0 n}+\phi_{n}
$$

and the equation for the perturbation stream function is

$$
\left(\frac{\partial}{\partial t}+U_{n} \frac{\partial}{\partial x}\right) q_{n}+\left[(-1)^{n} F_{n}\left(U_{1}-U_{2}\right)+\beta_{n}\right] \frac{\partial \phi_{n}}{\partial x}+J\left(\phi_{n}, q_{n}\right)=0
$$

where

$$
q_{n}=\nabla^{2} \phi_{n}+(-1)^{n} F_{n}\left(\phi_{1}-\phi_{2}\right)
$$

is the perturbation potential vorticity and $J\left(\phi_{n}, q_{n}\right)$ is the Jacobian of streamfunction and potential vorticity. The boundary conditions on the walls of the channel at $y=0,1$ are that the meridional velocity vanishes and the zonally averaged circulation on the two walls is conserved (Phillips, 1954) so that

$$
\frac{\partial \Psi_{n}}{\partial x}=0 \quad, \quad \frac{\partial}{\partial t} \int \frac{\partial \Psi_{n}}{\partial y} d x=0
$$

\subsection{Linear Stability Analysis}

We begin by addressing the linear stability problem in a similar fashion to Phillips (1954) and Pedlosky (1970). Wave solutions which satisfy the boundary conditions (7.8) can be found in the form

$$
\phi_{2}=\gamma \phi_{1}=\gamma A e^{i k(x-c t)} \sin (m \pi y)
$$

where $m$ is an integer, $k$ is the zonal wavenumber, $A$ is the amplitude of the wave in the upper layer, and $\gamma$ is the ratio between the amplitude of the perturbation in the lower to that of the upper layer. Only $k$ is restricted to be real. Substituting (7.9) 


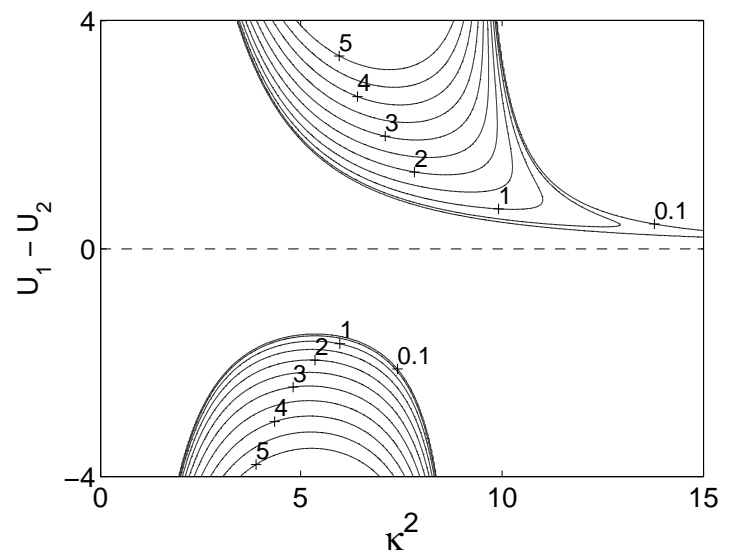

Figure 7.1: Stability curves for the two $\beta$ case for the vertical shear as function of the total wave number. The contours are of the growth rate in non-dimensional units, and the parameters used here are $F_{1}=100, F_{2}=20, \beta_{1}=10$, and $\beta_{2}=-30$.

into (7.6) and solving the linear eigenvalue problem gives the dispersion relation

$$
\begin{aligned}
c & =U_{2}+\frac{U_{S}}{2} \frac{\kappa^{2}+2 F_{2}}{\kappa^{2}+F_{1}+F_{2}}-\frac{\beta_{2}\left(\kappa^{2}+F_{1}\right)+\beta_{1}\left(\kappa^{2}+F_{2}\right)}{2 \kappa^{2}\left(\kappa^{2}+F_{1}+F_{2}\right)} \\
& \pm \frac{1}{2 \kappa^{2}\left(\kappa^{2}+F_{1}+F_{2}\right)}\left\{U_{S}^{2} \kappa^{4}\left(\kappa^{4}-4 F_{1} F_{2}\right)\right. \\
& +2 U_{S} \kappa^{2}\left[\left(\beta_{2}-\beta_{1}\right)\left(\kappa^{4}-2 F_{1} F_{2}\right)+\kappa^{2}\left(\beta_{2} F_{1}-\beta_{1} F_{2}\right)\right] \\
& \left.+\left[\left(\kappa^{2}+F_{1}\right) \beta_{2}-\left(\kappa^{2}+F_{2}\right) \beta_{1}\right]^{2}+4 F_{1} F_{2} \beta_{1} \beta_{2}\right\}^{\frac{1}{2}}
\end{aligned}
$$

where $U_{S}=U_{1}-U_{2}$ and $\kappa^{2}=k^{2}+l^{2}$ where $l=m \pi$. The solution also gives an expression for the ratio between the perturbation amplitude in each layer

$$
\gamma=1+\frac{\kappa^{2}}{F_{1}}-\frac{F_{1} U_{S}+\beta_{1}}{F_{1}\left(U_{1}-c\right)} .
$$

As seen in Figure 7.1 the short wave cut-off for classical two-layer baroclinic instabilities has disappeared and the marginal instability curve has a tail towards the high wave numbers. This effect does not require the bottom layer $\beta$ to be negative, only to differ from the upper one as shown by Steinsaltz (1987) for the case of a sloping bottom or by Robinson and McWilliams (1974) for a case of a varying bottom topography. However, the form of the potential vorticity (7.3) shows that for cases where the sign of $\beta$ is different in the two layers, the necessary condition for instability 
can be reached for arbitrarily weak shears, and analysis of (7.10) shows that this tail asymptotes to zero shear as $k \rightarrow \infty$ (Figure 7.1). Therefore, baroclinic instability may arise with the maximum growth rate at high wave numbers even when the shear is very small. As seen in Figure 7.1, the growth rate for a very small shear may itself be very small, and may seem insignificant, but since the observed zonal jets on the outer planets are long lived, an energy source from the weak instability may suffice.

The form of $c$ (7.10) is symmetric in the meridional and zonal wavenumber. Apri-

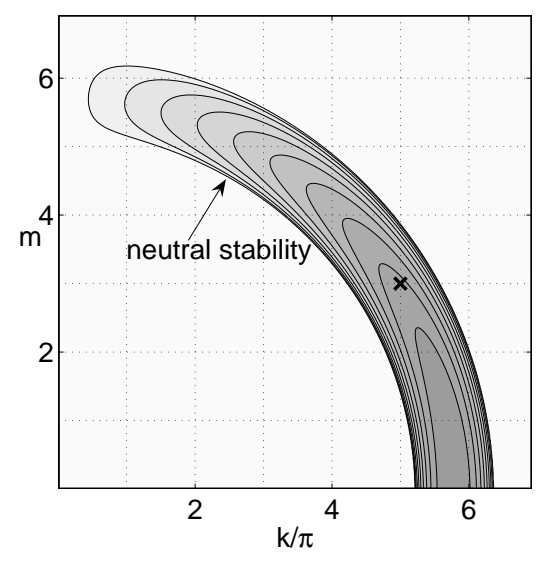

Figure 7.2: Growth rates in horizontal wave number space for the two $\beta$ case. The $x$ axis is the zonal wave number and the $y$ axis is the meridional one (divided by $\pi)$. The growth is confined to a band of few wave numbers. Due to the boundary conditions, the fastest growing mode (in this case $k_{m}=5, l_{m}=3$ - marked with an $\mathrm{x}$ ) is not necessarily the gravest mode. The parameters used here are $F_{1}=100, F_{2}=50$, $\beta_{1}=10, \beta_{2}=-30$ and $U_{S}=0.153$.

ori, one might think that the lowest meridional wave number for a given shear will be the most unstable (since the growth rate is $k c_{i}(\kappa)=\sqrt{\kappa^{2}-l^{2}} c_{i}(\kappa)$ ) so that the growth will not generate much meridional structure. However, the meridional and zonal wave numbers must be quantized as multiples of $\pi$ to satisfy the boundary condition in the channel, and for weak shears the band of unstable wave numbers is thinner than $\pi$ in wave number space. Thus for a given shear it may be that only high meridional wave numbers are unstable. This is demonstrated in Figure 7.2. 


\subsection{The Nonlinear Truncated Model}

The linear stability analysis implies that the short wave perturbations will become dominant for weak shears. In relation to the Jovian jets this implies the possibility of baroclinic instability creating a highly varying meridional structure. Of course, this must be tested in a full numerical model, and obvious questions are: can this meridional variation evolve into zonal jets? And if so, are the zonal velocities stable over time? In section 7.6 we use a full nonlinear numerical model to test this. However, before doing that, we can get some insights by solving the nonlinear system truncated to a perturbation in one wavenumber. Although this restricts the nonlinear nature of the solution, the band of initial growing modes in the two $\beta$ case is limited (Figure 7.2), so that this solution actually reproduces quite well (Figure 7.5) the initial stages of the fully nonlinear solution obtained numerically in section 7.6.

Therefore, we proceed to examine the nonlinear dynamics with taking the perturbation to have only one zonal wave number and one meridional wave number. Rewriting (7.6) in terms of the barotropic $\Pi_{T}, \Psi_{T}$ and baroclinic $\Pi_{C}, \Psi_{C}$ components gives

$$
\begin{aligned}
\frac{\partial}{\partial t} \Pi_{T}+J\left(\Psi_{T}, \Pi_{T}\right)+J\left(\Psi_{C}, \Pi_{C}\right) & =0 \\
\frac{\partial}{\partial t} \Pi_{C}+J\left(\Psi_{T}+\xi \Psi_{C}, \Pi_{C}\right)+J\left(\Psi_{T}, \Pi_{C}\right) & =0
\end{aligned}
$$

where the barotropic and baroclinic components of the potential vorticity are

$$
\begin{aligned}
\Pi_{T} & =\frac{\delta \Pi_{1}+\Pi_{2}}{1+\delta} \\
\Pi_{C} & =\frac{\sqrt{\delta}}{1+\delta}\left(\Pi_{1}-\Pi_{2}\right)
\end{aligned}
$$

and $\delta=\frac{D_{1}}{D_{2}}$ is the layer depth ratio. The same structure applies for the barotropic and baroclinic stream functions $\Psi_{T}$ and $\Psi_{C}$. The parameter $\xi=\frac{(1-\delta)}{\sqrt{\delta}}$ comes from the unequal upper and lower layer thicknesses. Split into a basic state and a perturbation and using (7.3) and (7.4), the barotropic and baroclinic potential vorticities and streamfunctions are 


$$
\begin{aligned}
\Psi_{T} & =\phi_{T} \\
\Psi_{C} & =-U_{C} y+\phi_{C} \\
\Pi_{T} & =\frac{\left(\delta \beta_{1}+\beta_{2}\right) y}{1+\delta}+q_{T} \equiv Q_{T} y+q_{T} \\
\Pi_{C} & =\left[\frac{\sqrt{\delta}}{1+\delta}\left(\beta_{1}-\beta_{2}\right)+\left(F_{1}+F_{2}\right) U_{C}\right] y+q_{C} \equiv Q_{C} y+q_{C}
\end{aligned}
$$

where $U_{C}$ is the baroclinic shear, and $\phi$ and $q$ are the perturbation stream function and potential vorticity respectively. Note that the basic state barotropic streamfunction has been taken to be zero. This can be done due to the Galilean invariance of the two-layer system. Then the barotropic and baroclinic equations $(7.12,7.13)$ take the form

$$
\begin{aligned}
\frac{\partial}{\partial t} q_{T}+U_{C} \frac{\partial}{\partial x} q_{C}+Q_{T} \frac{\partial}{\partial x} \phi_{C}+Q_{C} \frac{\partial}{\partial x} \phi_{C} & \\
+J\left(\phi_{T}, q_{T}\right)+J\left(\phi_{C}, q_{C}\right) & =0 \\
\frac{\partial}{\partial t} q_{C}+U_{C}\left(\frac{\partial}{\partial x} q_{T}+\xi \frac{\partial}{\partial x} q_{C}\right)+Q_{T} \frac{\partial}{\partial x} \phi_{T} & \\
+Q_{C}\left(\frac{\partial}{\partial x} \phi_{T}+\xi \frac{\partial}{\partial x} \phi_{C}\right)+J\left(\phi_{T}+\xi \phi_{C}, q_{C}\right)+J\left(\phi_{C}, q_{T}\right) & =0
\end{aligned}
$$

We express the solution as a single potential vorticity perturbation wave which satisfies the boundary conditions (7.8) of the form

$$
\begin{aligned}
& q_{T}=q_{T}^{\prime}(t) e^{i k x} \sin (l y)+c . c . \\
& q_{C}=q_{C}^{\prime}(t) e^{i k x} \sin (l y)+c . c .
\end{aligned}
$$

and then the perturbation stream functions can be expressed via the inversion relation so that

$$
\begin{aligned}
\phi_{T} & =\frac{-q_{T}}{\kappa^{2}} \\
\phi_{C} & =\frac{-q_{C}}{\kappa^{2}+F_{1}+F_{2}}
\end{aligned}
$$


The main advantage of writing the quasi geostrophic potential vorticity equations in this form is that when plugging (7.22-7.25) into $(7.20,7.21)$ the Jacobians from the barotropic equation (7.20) vanish, while the baroclinic nonlinear contribution (7.21) gives

$$
\begin{array}{r}
J\left(\phi_{T}, q_{C}\right)+J\left(\phi_{C}, q_{T}\right)= \\
\frac{i k l\left(F_{1}+F_{2}\right)}{\kappa^{2}\left(\kappa^{2}+F_{1}+F_{2}\right)}\left[q_{C}^{\prime} q_{T}^{*}-q_{C}^{\prime *} q_{T}^{\prime}\right] \sin (2 l y)
\end{array}
$$

This is where the truncated nature of the solution appears. The nonlinear baroclinic interaction gives a zonal mean correction to the basic flow with a specific meridional structure which depends on the choice of the truncated mode. Nevertheless, as mentioned in the linear analysis (which applies when the perturbation is small) since the band of growing modes contains only few modes (Figure 7.2) an approximation of only one growing mode turns out to be a fair approximation. Since the basic zonal flow is fixed, we can specify this mode to be the fastest growing mode. Therefore we can split the baroclinic equation (7.21) in two: one part for the linear perturbation, and another for the nonlinear correction. From the solution to the nonlinear part (7.26) we can approximate the structure of the nonlinear correction to the potential vorticity as having the form

$$
\overline{q_{C}}=\overline{q_{C}^{\prime}}(t) \sin (2 l y) .
$$

This form is unlike the linear perturbation part $(7.7,7.9)$, having no zonal dependence and a different meridional structure. This nonlinear correction to the basic baroclinic state must also satisfy the two boundary conditions given by (7.8). In order to ensure this, we use the inversion relation from (7.7) for the zonally averaged case

$$
\left[\frac{\partial^{2}}{\partial y^{2}}-\left(F_{1}+F_{2}\right)\right] \overline{\phi_{C}}=\overline{q_{C}}
$$

which, when solved for $\overline{\phi_{C}}$ with the boundary conditions, gives a correction to the 
basic zonal velocity

$$
\begin{aligned}
\overline{U_{C}}= & \frac{2 l \overline{q_{C}^{\prime}}(t)}{4 l^{2}+F_{1}+F_{2}} \\
& \cdot\left[\cos (2 l y)-\frac{\cosh \left[\sqrt{F_{1}+F_{2}}\left(y-\frac{1}{2}\right)\right]}{\cosh \left(\frac{\sqrt{F_{1}+F_{2}}}{2}\right)}\right]
\end{aligned}
$$

This result is similar in form to that found for the weakly nonlinear theory by Pedlosky (1970). Here though, the weakly nonlinear requirement is relaxed (but replaced by a truncation assumption) and this correction may extend into the highly supercritical regime, as we show in the numerical experiments in section 7.6. The amplitude of this zonal flow is not limited to the weakly varying parameter and, in fact, can be stronger than the eddies themselves.

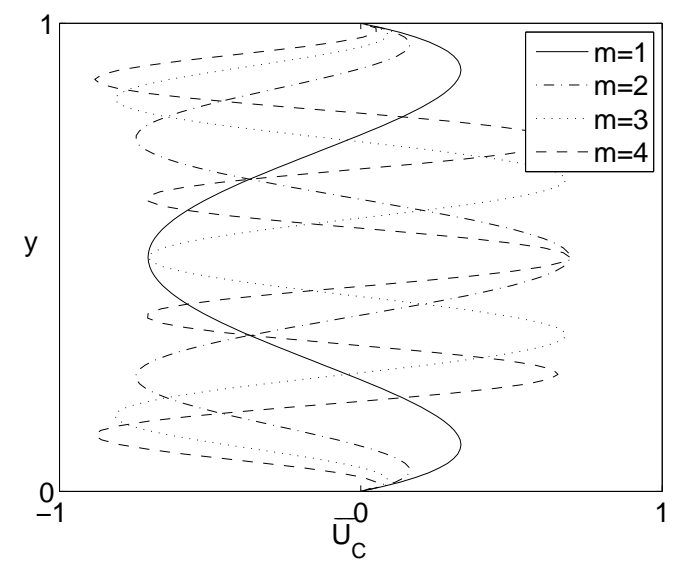

Figure 7.3: The analytical baroclinic induced zonal velocity $\overline{U_{C}}(7.29)$ from the truncated model as function of the channel width for the first four meridional modes.

Figure 7.3 shows the shape of the mean flow correction for the first few modes, and indicates that for the higher modes we expect to get a multi-jet meridional structure. This baroclinic contribution tends to reduce the shear rather than increase it, causing oscillations in the amplitude of the perturbation in the classical weakly supercritical case (Pedlosky, 1970); once the correction reduces the shear enough it goes back into the stable regime, halting the growth until the effect of the nonlinearities decreases, and the cycle repeats. Here, since the flow may be strongly rather than weakly supercritical the nonlinear wave effects may not be enough to halt the growth. For the cases of high wave number perturbations, though, the growth band (Figure 
7.2) becomes narrow in wave space so that the effect of the nonlinear correction is similar to that of the weakly nonlinear case, and the perturbation may reach a steady equilibrium. These oscillations can be seen in Figure 7.4.

The truncation and the separation of the nonlinear part out of the baroclinic equation allows us not only to find the baroclinic induced zonal velocity $\overline{U_{C}}$, but to solve for the perturbation amplitudes and the baroclinic mean. We can write the truncated system as a closed system of three equations for three unknowns; the perturbation amplitudes $q_{T}^{\prime}, q_{C}^{\prime}$ and the baroclinic mean $\overline{q_{C}^{\prime}}$. The specifics of this derivation are given in Appendix (B.1). The resulting system is

$$
\begin{aligned}
\frac{\partial}{\partial t} \mathbf{q}+i k \mathbf{L q}+i k \overline{q_{C}^{\prime}} \mathbf{N q} & =0 \\
\frac{\partial}{\partial t} \overline{q_{C}^{\prime}}+\frac{i k l\left(F_{1}+F_{2}\right)}{\kappa^{2}\left(\kappa^{2}+F_{1}+F_{2}\right)}\left[q_{C}^{\prime} q_{T}^{*}-q_{C}^{\prime} q_{T}^{\prime}\right] & =0
\end{aligned}
$$

where $\mathbf{q}=\left(\begin{array}{c}q_{T}^{\prime} \\ q_{C}^{\prime}\end{array}\right)$ and the operators $\mathbf{N}$ and $\mathbf{L}$ are given in Appendix (B.1) as well. This solution is shown in Figure 7.4 which plots the evolution of enstrophy in time for the linear case, the truncated nonlinear case, and a full nonlinear model containing many modes (section 7.6). This example shows how the nonlinearities stabilize the initial instability in both the truncated and full model.

Since the initial perturbation is small, and the system is baroclinically unstable, the perturbations in all models grow similarly. When the effect of the nonlinearities is large enough, the nonlinear models separate from the linear model and, since the perturbation is dominated by the most rapidly growing mode, the truncated model with only this mode gives a reasonable estimate of this separation point. Then the truncated model begins to oscillate by exchanging energy between the perturbations and the basic flow, whereas the full model (which resolves harmonics neglected in the truncation) equilibrates with a much more steady amplitude. In general this truncated solution captures well when, where, and how the interaction with the mean flow halts the instability. We have seen a somewhat similar interaction between the nonlinearities and the mean flow in the convection model (section 5.1). There, the nonlinear eddy fluxes induced a mean zonal velocity and then acted to exchange energy between the upgradient momentum fluxes and the viscous fluxes (see Figures 5.1 and 5.2). 


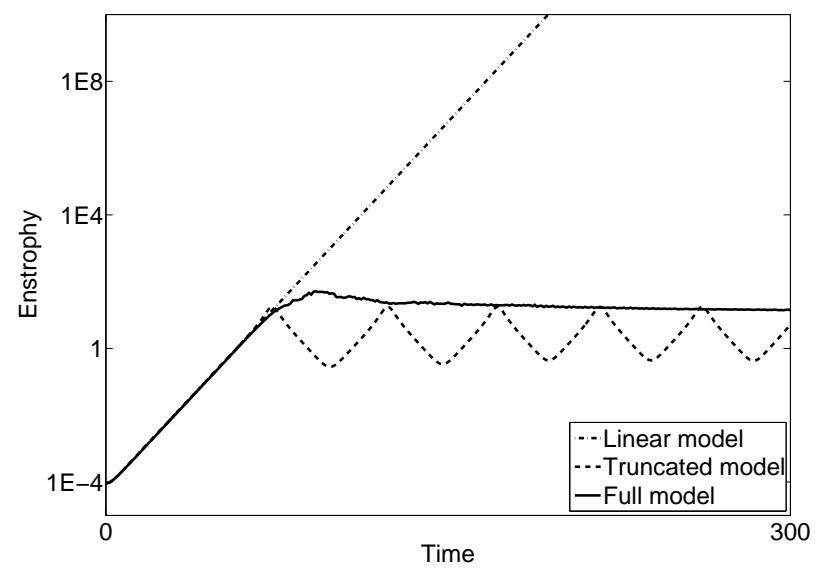

Figure 7.4: The enstrophy as a function of time. The dash-dot line is the linear growth rate for the fastest growing mode using the linear growth rate from (7.10). The dashed line is the growth calculated from the truncated model (7.30 and 7.31). This shows that when the perturbation is small the system aligns with the linear growth rate, until the nonlinear terms become dominant and the system begins oscillating while exchanging energy between the eddies and the mean flow. The solid line is the result for the full nonlinear system (run S4) which qualitatively follows the truncated model but contains many modes and therefore does not have a pure oscillation .

In summary, the truncated model allows us to examine qualitatively the nonlinear interactions which have several roles. First, they create an induced zonal flow with a highly varying meridional structure which (as we show in section 7.6) may be stronger than the eddies and therefore have the potential of becoming zonal jets. Second, this induced flow stabilizes the growing perturbations. This "toy model" provides a closed system of equations for the perturbation amplitude in both layers and the change in the basic flow due to nonlinearities, without requiring the system to be only slightly supercritical; for such cases the correction due to the nonlinearities becomes significant (as opposed to being on the order of the departure from the critical curve) and a strong multi-jet structure may emerge. Indeed, since the high wave number instability dominates the two $\beta$ case, we might expect multiple jets for a weak baroclinic shear. 


\subsection{The Weakly Supercritical Limit}

Most previous treatments of nonlinear baroclinic instability have required the system to be weakly supercritical (e.g. Pedlosky, 1970). Instead, we have truncated the system to one unstable mode. Here we present the truncated model in the limit where the shear is taken to be just slightly supercritical. This limit corresponds to the weakly supercritical theory of Pedlosky (1970), except that we allow for the more general case of different layer depths and a variable $\beta$. If we vary the value of the critical shear by a small parameter $\Delta$, so that it slightly exceeds the critical value

$$
U_{S}=U_{c}+\Delta
$$

then the imaginary part of the linear growth rate (7.10) becomes

$$
\begin{aligned}
c_{i} & =\frac{\Delta^{\frac{1}{2}}}{\sqrt{2} \kappa\left(\kappa^{2}+F_{1}+F_{2}\right)}\left\{U_{C} \kappa^{4}\left(\kappa^{4}-4 F_{1} F_{2}\right)\right. \\
& \left.+\left[\left(\beta_{2}-\beta_{1}\right)\left(\kappa^{4}-2 F_{1} F_{2}\right)+\kappa^{2}\left(\beta_{2} F_{1}-\beta_{1} F_{2}\right)\right]\right\}^{\frac{1}{2}}
\end{aligned}
$$

Thus it is proportional to $\Delta^{\frac{1}{2}}$. Therefore we follow Pedlosky (1970) and define a slow time scale, $T$, such that

$$
\frac{\partial}{\partial t} \rightarrow \frac{\partial}{\partial t}+\Delta^{\frac{1}{2}} \frac{\partial}{\partial T}
$$

With these expansions we are able to obtain an analytic solution to the system of equations $(7.30,7.31)$. The small variation to the shear $U_{0} \rightarrow U_{0}+\Delta$ leads to an expansion of the operators in (7.30)

$$
\mathbf{L}=\mathbf{L}_{0}+\Delta \mathbf{L}_{2}
$$

and we assume the system is weakly nonlinear so that $\mathbf{N}$ will be $O(\Delta)$. The operators themselves are given in Appendix (B.2). We expand the potential vorticity perturbation (7.7) as well

$$
q=e^{-i k c_{0} t}\left[q_{0}+\Delta^{\frac{1}{2}} q_{1}+\Delta q_{2}+O\left(\Delta^{\frac{3}{2}}\right)\right]
$$


By the choice of $c_{0}$ as the neutral phase speed $\mathbf{q}_{0}$ does not depend on $t$. Expanding (7.30) in powers of $\Delta^{\frac{1}{2}}$, gives

$$
\begin{aligned}
O\left(\Delta^{\frac{1}{2}}\right): & : i k\left(\mathbf{L}_{0}-c_{0} \mathbf{I}\right) \mathbf{q}_{0}=0 \\
O(\Delta): & \frac{\partial}{\partial t} \mathbf{q}_{1}+i k\left(\mathbf{L}_{0}-c_{0} \mathbf{I}\right) \mathbf{q}_{1}+\frac{\partial}{\partial T} \mathbf{q}_{0}=0 \\
O\left(\Delta^{\frac{3}{2}}\right): & \frac{\partial}{\partial t} \mathbf{q}_{2}+i k\left(\mathbf{L}_{0}-c_{0} \mathbf{I}\right) \mathbf{q}_{2}+\frac{\partial}{\partial T} \mathbf{q}_{1} \\
& +i k\left(\mathbf{L}_{2}+\mathbf{N} \overline{q_{C}^{\prime}}\right) \mathbf{q}_{0}=0
\end{aligned}
$$

Solving this system with the equation for the mean baroclinic correction (full solution in Appendix (B.2)) gives an amplitude equation for the growth of the perturbation

$$
\frac{\partial^{2} A}{\partial T^{2}}-k^{2} c_{i}^{2} A+k^{2} N A\left(|A|^{2}-|A(0)|^{2}\right)=0
$$

where $A$ is the amplitude of the perturbation and the nonlinear parameter $N$ is the Landau coefficient which is given also in Appendix (B.2). For very small amplitudes the system thus reduces to the linear system. As the amplitude grows the cubic term becomes more dominant and if $N>0$ this term will act to slow the growth and eventually reverse it. At a certain value of $A$ this term will change sign and begin increasing the growth, and thus a limit cycle is created. This type of oscillation is seen in Figure 7.4.

\subsection{Fully Nonlinear Model and the Generation of Multiple Zonal Jets}

The truncated model predicts a multi-jet structure for high wave number instability. In this section we use a fully nonlinear numerical model to explore the role of the other modes on the generation of eddies and jets, and on the effect of quasi-geostrophic turbulence on these jets. The model we use is based on the same equations analyzed in the previous sections. It is pseudo-spectral (Boyd, 2001), where each layer has a spatial resolution of $64 \times 128$, is periodic in the zonal direction, and is confined within a channel in the meridional direction. On the channel walls we require no meridional flow and that the circulation is conserved (implemented by requiring the 
mean ageostrophic meridional velocity to vanish).

The parameter regime is fairly simple since we only set the layer depths (by setting the nondimensional Froude numbers), the $\beta$ parameters and the baroclinic shear. If we were to fully compare the numerical model to the truncated model we would set the shear in such a way that only one mode will be growing (see Figure 7.2). For our standard run, following Dowling and Ingersoll (1989) and Ingersoll and Pollard (1982) we choose the typical Rossby deformation radius to be on the order of 2000 $\mathrm{km}$. This value corresponds to the observed scale of the jets on Jupiter. We take the domain width to be an order of magnitude bigger then the deformation radius, thus setting the upper nondimensional Froude number to be $F_{1}=100\left(F_{n}=\frac{L^{2}}{L_{D}^{2}}\right)$. The bottom layer is taken as to be 5 times as deep so that $F_{2}=20 . \beta_{1}$ is set according to the curvature of Jupiter $\left(\beta_{1}=\frac{2 \Omega \cos \theta}{R_{J}} \frac{L^{2}}{U}\right)$ giving the nondimensional value $\beta_{1}=10$, with the characteristic velocity being $50 \mathrm{~m} / \mathrm{s}$ and the same typical horizontal length scale of $2 \mathrm{E} 4 \mathrm{~km}$. Following the barotropic stability analysis by Ingersoll and Pollard (1982) which shows that $\beta_{2}$ is at least $-3 \beta_{1}$ we set $\beta_{2}$ to this value. Their analysis shows that this is a lower limit for stability and in fact a more negative lower layer $\beta$ will be stable, but for our standard run we choose this limit. Unlike other models for jets (Williams, 1979; Panetta, 1993; Vallis and Maltrud, 1993) we find in the upper layer that the standard barotropic stability criterion Kuo (1949) is violated (Figure 7.14), much as we see in the observations, but the flow is still stable. We refer to these values as our standard run (denoted with $\mathrm{S}$ and the run number - see Table 7.1), and experiment sets B and F show a sensitivity analysis to the parameters of the standard run. The vertical shear is set so that several growing unstable modes exist, as demonstrated in Figures 7.1 and 7.2. Since the two layer model is invariant under translation (Pedlosky, 1987) it is only necessary to set the baroclinic shear $U_{1}-U_{2}$ and not the absolute values of the basic state velocities.

We begin all our experiments with a small random potential vorticity perturbation field, with initial perturbations in all $\pi$ multiple wave numbers up to $k, l=10 \pi$. Since the system is forced by a constant vertical shear, eventually the system becomes baroclinically unstable and the fastest growing mode dominates. We denote this fastest growing mode with the notation $k_{m}, l_{m}$ such that $k=k_{m} \pi$ and $l=l_{m} \pi$. As seen in Figure 7.4 the enstrophy begins growing in agreement with linear theory (since the amplitude is small). When the effects of nonlinearity grow enough, the enstrophy diverges from the theoretical linear growth curve as predicted by the truncated model. 

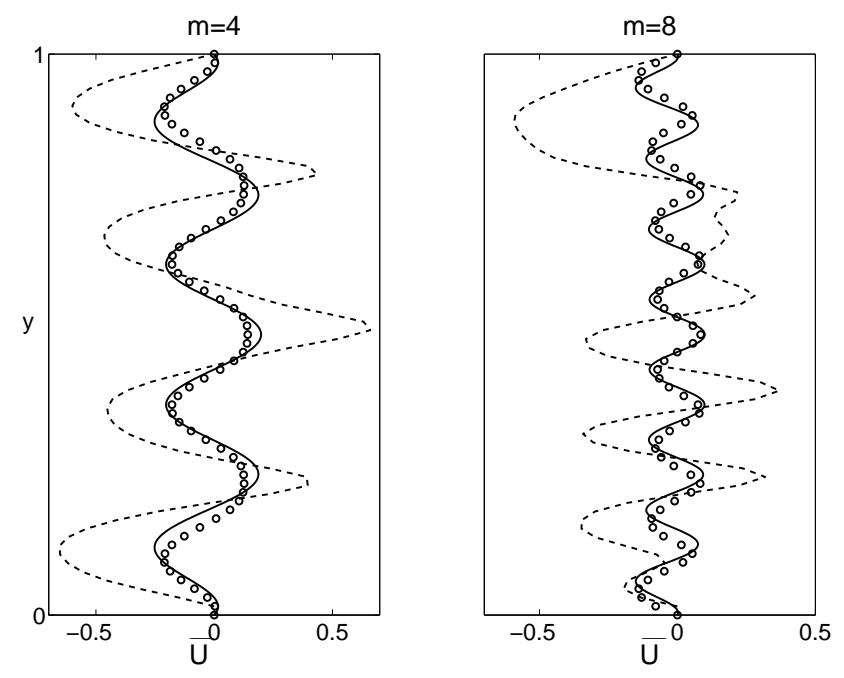

Figure 7.5: The induced zonal velocity as a function of the channel width. The solid line is the theoretical baroclinic correction $\overline{U_{C}}$ (7.29) from the truncated model; the circles are the result from the nonlinear numerical model (the 65 points are the meridional resolution of the grid) towards the end of the baroclinic growth stage for $\overline{U_{1}}$, and the dashed line is the steady state of the numerical results after the inverse energy cascade.

It can be seen that the nonlinear truncated model predicts quite precisely where this separation takes place. Moreover, when we plot a snapshot of the top layer induced zonal flow $\overline{U_{1}}$ at this time from the numerical experiments (circles in Figure 7.5), it matches well the truncated model theoretical prediction. The reason for this is that as long as the growth of the perturbation is dominated by the fastest growing mode according to the truncated model there is no induced barotropic velocity. From the form of $(7.14,7.15)$ we can write the induced zonal velocity in each layer

$$
\overline{U_{1}}=\frac{1}{\sqrt{\delta}} \overline{U_{C}}+\overline{U_{T}} \quad \overline{U_{2}}=-\sqrt{\delta} \overline{U_{C}}+\overline{U_{T}}
$$

and therefore the induced zonal flow in each layer has the same structure as the baroclinic induced zonal flow $\overline{U_{C}}$. We see exactly a ratio of $\delta$ between of the amplitude of the induced zonal flow in the upper and lower layers.

In Figure 7.5 the analytic result of equation (7.29) is plotted for the cases of meridional wave numbers $m=4,8$ with the results from the full model for runs S2 and S14. The numerical results contain 65 points (the meridional resolution of the 


\begin{tabular}{|c|c|c|c|c|c|c|c|c|c|}
\hline Run & $F_{1}$ & $1 / \delta$ & $\beta_{1}$ & $B$ & $U_{S}$ & $k_{m}, l_{m}$ & $1 / \lambda$ & $\eta_{1}$ & $\eta_{2}$ \\
\hline S1 & 100 & 5 & 10 & -3 & 0.0263 & 10,8 & 7 & 1.87 & 0.11 \\
\hline S2 & & & & & 0.0296 & 9,8 & 8.9 & 2.32 & 0.13 \\
\hline S3 & & & & & 0.0332 & 9,7 & 6.37 & 3.1 & 0.15 \\
\hline $\mathrm{S} 4$ & & & & & 0.0338 & 8,7 & 7.09 & 3.17 & 0.13 \\
\hline S5 & & & & & 0.0372 & 10,4 & 6.25 & 3.33 & 0.13 \\
\hline S6 & & & & & 0.0385 & 8,7 & 5.79 & 2.54 & 0.17 \\
\hline S7 & & & & & 0.0413 & 10,3 & 7.53 & 1.56 & 0.11 \\
\hline S8 & & & & & 0.044 & 8,6 & 5.44 & 3.18 & 0.25 \\
\hline S9 & & & & & 0.045 & 8,7 & 7.71 & 3.46 & 0.15 \\
\hline S10 & & & & & 0.0455 & 9,4 & 4.14 & 3.26 & 0.17 \\
\hline S11 & & & & & 0.0494 & 8,4 & 6.88 & 3.07 & 0.19 \\
\hline S12 & & & & & 0.05 & 9,3 & 6.14 & 1.35 & 0.14 \\
\hline S13 & & & & & 0.0525 & 9,2 & 4.28 & 3.2 & 0.28 \\
\hline S14 & & & & & 0.0562 & 8,4 & 6.5 & 2.94 & 0.22 \\
\hline S15 & & & & & 0.0612 & 7,5 & 6.92 & 1.94 & 0.36 \\
\hline S16 & & & & & 0.0622 & 8,3 & 5.63 & 2.36 & 0.31 \\
\hline S17 & & & & & 0.0632 & 7,3 & 5.76 & 2.07 & 0.25 \\
\hline B1 & 100 & 5 & 10 & -10 & 0.1032 & 10,4 & 8.56 & 2.27 & 0.1 \\
\hline B2 & & & & -5 & 0.056 & 10,4 & 7.33 & 1.83 & 0.11 \\
\hline B3 & & & & -2 & 0.0277 & 9,6 & 7.26 & 1.63 & 0.15 \\
\hline B4 & & & & -1 & 0.0183 & 9,6 & 5.92 & 0.73 & 0.16 \\
\hline B5 & & & & 0 & 0.0088 & none & no jets & 0 & 0 \\
\hline B6 & & & & 1 & 0.0006 & none & no jets & 0 & 0 \\
\hline F1 & 100 & 1 & 10 & -3 & 0.0382 & 10,4 & 6.15 & 0.48 & 0.21 \\
\hline F2 & & 10 & & & 0.037 & 9,7 & 10.25 & 2.78 & 0.11 \\
\hline F3 & & 100 & & & 0.0369 & 8,5 & 6.04 & 0.51 & 0.03 \\
\hline
\end{tabular}

Table 7.1: Numerical experiments using the fully nonlinear pseudo-spectral model. $F_{1}$ is the Froude number for the upper layer; $\delta$ is the layer depth ratio between the upper and lower layer (and inverse of the Froude number ratio); $\beta_{1}$ is the $\beta$-plane parameter for the upper layer; $B$ is the ratio $\frac{\beta_{2}}{\beta_{1}} ; U_{S}$ is the imposed baroclinic shear; $k_{m}, l_{m}$ are the fastest growing baroclinic modes; $\lambda$ is the meridional spectral maximum of the statistically steady state averaged across the channel (thus $\frac{1}{\lambda}$ gives an estimate for the average number of jets), and $\eta$ is the ratio of the induced zonal velocity amplitude to the eddy amplitude (see text) in each layer. 
model) and are a snapshot of $\overline{U_{1}}$ (which has the same structure as the $\overline{U_{C}}$ field (7.41)) taken just before the time when the two models diverge. Therefore, although the
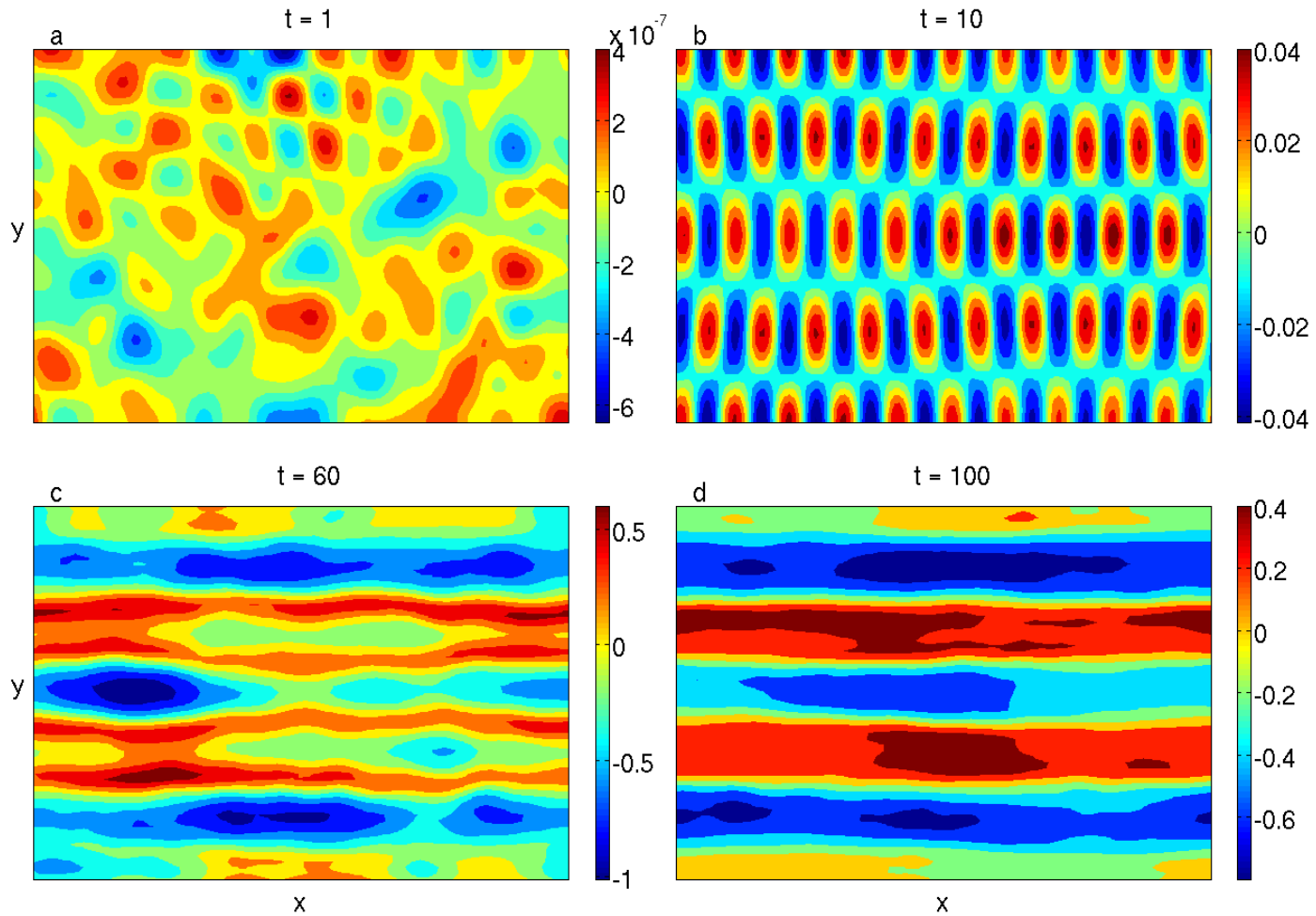

Figure 7.6: Instantaneous total zonal velocity fields at different times for the top layer (run S5). Beginning with a random vorticity perturbation (a), then becoming baroclinically unstable (b) dominated by the fastest growing mode (in this case $k_{m}=$ $10, l_{m}=4$ ), then several jets are formed (c) matching the prediction of the truncated model, and cascading to stable jets (d) with a typical width on the order of the Rhines scale. Full simulations of the zonal velocity field for this run are available at http://lake.mit.edu/ glenn/yohai/movies.html

choice of only one mode in the truncated model seems initially quite restrictive for a nonlinear prediction, in this type of instability scenario where the fastest growing mode dominates until turbulence develops, the truncation is quite useful. After the models diverge and more modes come into play, the truncated and the full numerical models differ in the sense that there is no pure oscillation in enstrophy in the full model as in the truncated model (although we can create such oscillations for special weakly nonlinear cases), but rather a noisier (higher frequency) signal (Figure 7.4). However, the amplitude in the two models is of the same order. Qualitatively, this equilibrium state is the same as seen in the truncated model for one mode, except 

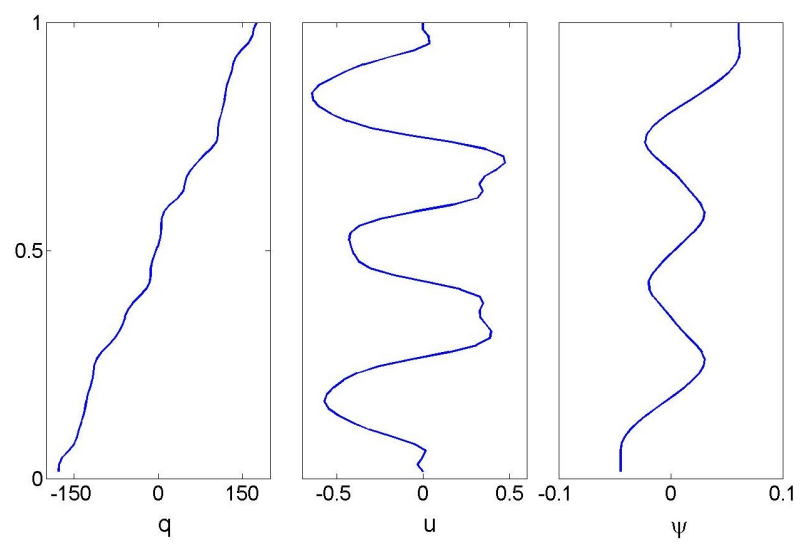

Figure 7.7: Zonally averaged fields for the top layer at steady state (run S5). left: potential vorticity $\left(q_{1}\right)$; center: total zonal velocity $\left(u_{1}\right)$; right: streamfunction $\left(\psi_{1}\right)$

that, as the energy cascades more modes appear, and the combination of them all creates this leveling of the enstrophy. Figure 7.5 then also shows the final steady state after the inverse cascade, showing distinct jets with a scale set by the baroclinic induced zonal velocity $\overline{U_{C}}$. The jets have sharper eastward than westward winds, due to the asymmetry in the barotropic stability criterion (Figure 7.14).

The total zonal velocity in each layer is composed of three components; the constant basic flow creating the vertical shear, the induced zonal velocity created by the nonlinear interaction $\overline{U_{n}}$ and the $u_{n}^{\prime}$ eddy field . As the instability grows, the induced zonal flow grows by many orders of magnitude and, as discussed above, forms into a multi-jet structure. Once the growth is halted, and the enstrophy settles into equilibrium, quasi-geostrophic turbulence causes the mean horizontal scales to increase. The inverse energy cascade also affects the jets and the initial multi-jet structure (which so far was determined only by the dominant growing mode) breaks down; then fewer but stronger jets appear (Figure 7.11).

An example of the formation of jets is presented in Figure 7.6 which shows snapshots of the zonal velocity field of the top layer (without the constant applied velocity $U_{1}$ to emphasis the change of the amplitude following the instability) at different times. Initially a small random perturbation is applied to the basic state (7.6a). At some time the fastest growing mode (in this case $k_{m}=10, l_{m}=4$ ) becomes dominant (7.6b) and the perturbation grows exponentially. Then, as predicted by the truncated model, the nonlinear interactions form several jets (7.6c). In time, more modes come in, the flow becomes turbulent, and an inverse energy cascade begins setting five ma- 

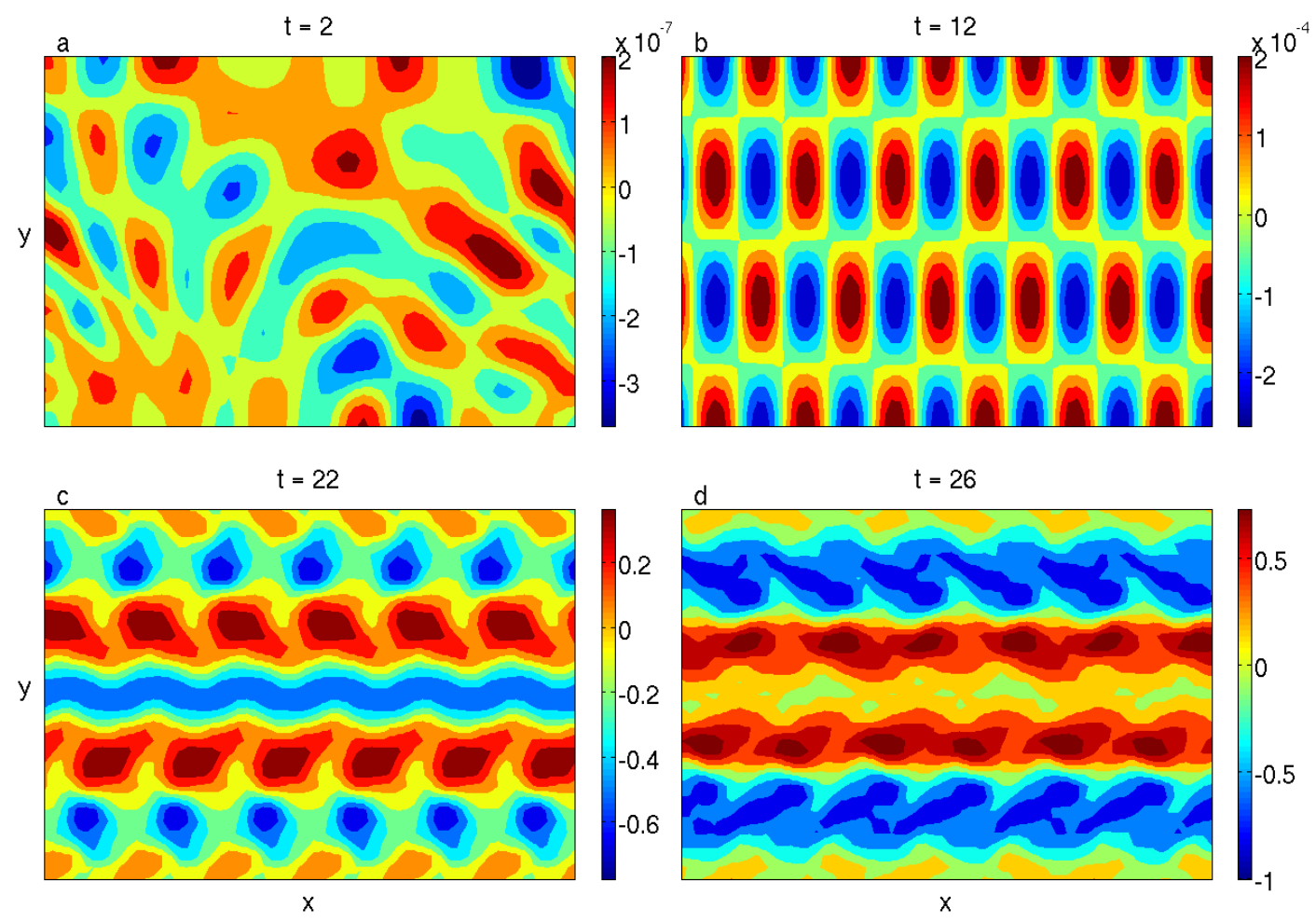

Figure 7.8: The transition from baroclinic instability to jets: Instantaneous total zonal velocity fields at different times for the top layer. These snapshots "zoom in" on the transition from the growing baroclinic perturbation to jets; beginning from a weak random eddy field (a) to a growing baroclinic perturbation (b) and transitioning to a jet structure (c) cascading to larger meridional scales (d). Full simulations of the zonal velocity field for this run are available at http://lake.mit.edu/ glenn/yohai/movies.html

jor jets (7.6d) in the channel (two westerly and three easterly) with a typical width on the order of the Rhines scale (Figure 7.9). Figure 7.10 includes arrows for the total velocity for the same run (S5), indicating the dominance of the zonal velocities over the meridional velocities. In Figure 7.7 we show the top level zonal mean potential vorticity, streamfunction and zonal velocity for the same run presented in Figure 7.6 (S5) at steady state. It shows the potential vorticity is dominated by the background component, but the signature of the potential vorticity staircase is apparent.

Figure 7.8 shows a similar plot to the one in Figure 7.6 but for a case of higher vertical shear, which grows in a lower wave number $\left(k_{m}=6, l_{m}=3\right)$ and cascades rather quickly into 5 jets and then one wide central westerly jet with two narrower easterly jets. The snapshots in this case are closer in time and show the transition 


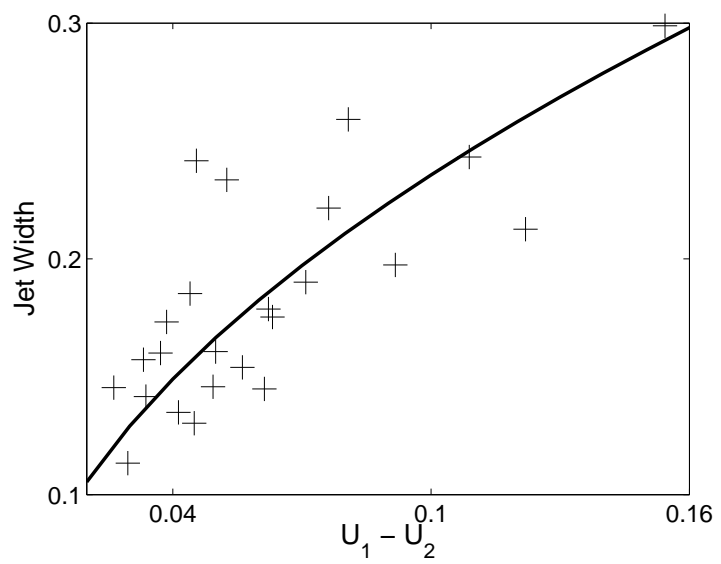

Figure 7.9: The jet width as a function of the applied shear. The marks denote the average width of the jets in the top layer for each standard run. The width of the jet is determined by a spectral analysis of the final statistical steady state, and averaging the leading meridional wave length across the channel. The solid line is the theoretical value for the Rhines scale using the non-dimensional shear and the $\beta$ value for the upper layer.

from a linearly growing disturbance into a strong zonal jet.

Geostrophic turbulence theory predicts that after the system becomes turbulent the typical scale will cascade up to the Rhines scale $L_{\beta}=\pi \sqrt{\frac{2 U}{\beta}}$ (Rhines, 1975). The relation of this scale to the meridional scale of the Jovian jets has been proposed by Williams (1979). It can be seen in Table 7.1 that our numerical results agree with this scaling, since the lower the shear the higher the mode of the fastest growing mode might be, and then more initial jets may be formed. Typically, more jets at the initial stages result in more jets at equilibrium after the inverse energy cascade. Figure 7.9 shows the mean typical scale of the jets as function of the shear for all standard runs (some presented in Table 7.1). The width of the jet is determined by doing a spectral analysis of the final statistical steady state for each run and averaging the leading meridional wave length across the channel. We can see that in general the final scale is governed by the Rhines scale.

Figure 7.10 shows the final zonal state for a few of the experiments shown in Table 7.1. The experiments differ in the applied shear which sets a different induced meridional structure resulting in a different statistical steady state after the energy cascade. An example for the evolution of the $\overline{U_{1}}$ field in time is given in Figure 7.11.

In all experiments the nonlinear correction $\overline{U_{n}}$ which is initially weak (due to 

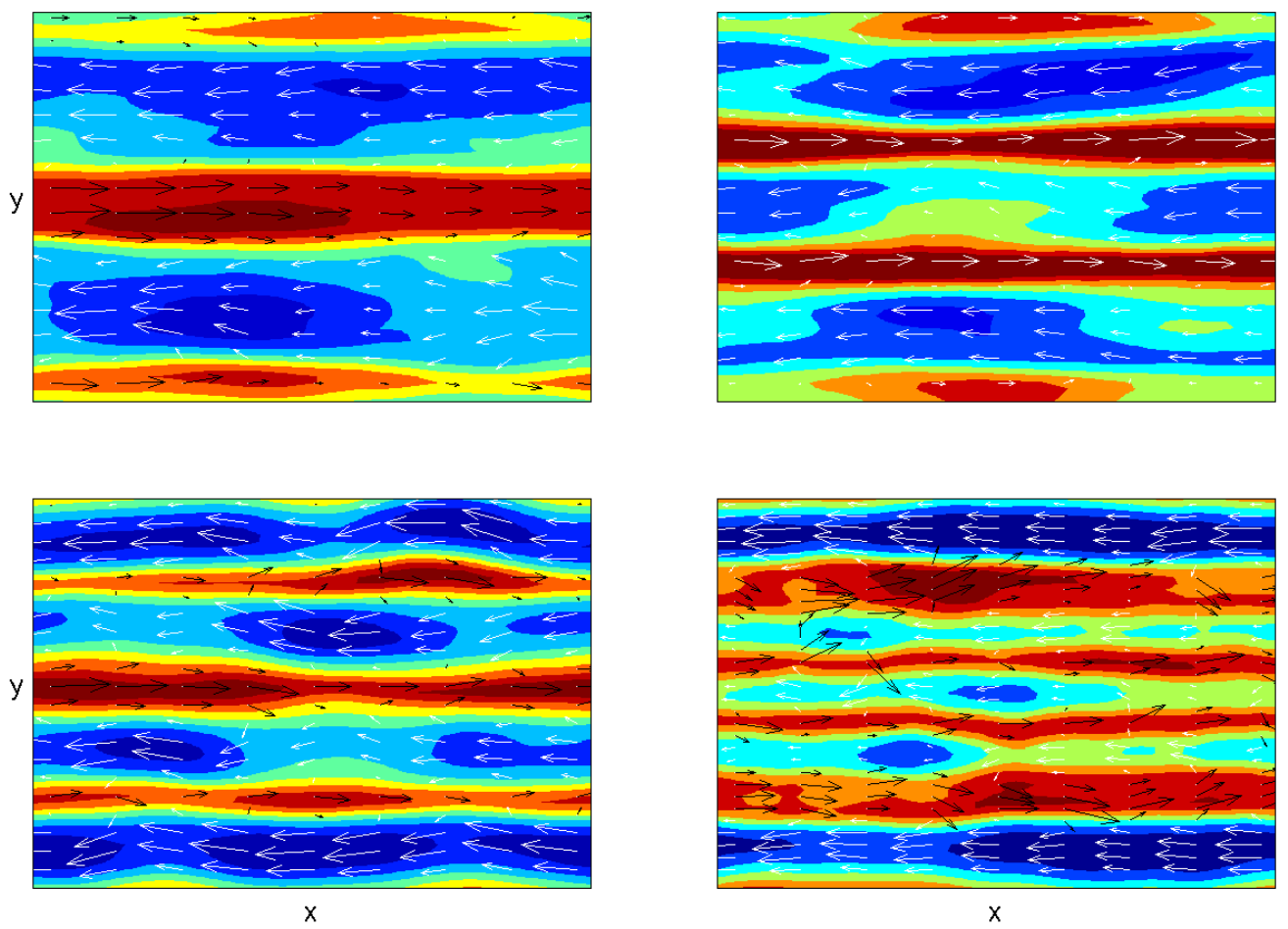

Figure 7.10: Examples of the final steady state total velocity fields for different runs. The contours are the zonal velocity and arrows show the total velocity (zonal and meridional). The upper left panel is run S13 with an applied shear of $U_{S}=0.0525$ and fastest growing mode $k_{m}=10, l_{m}=8$; upper right panel is run S5 with an applied shear of $U_{S}=0.0372$ and fastest growing mode $k_{m}, l_{m}=10,4$; lower left panel is run S14 with an applied shear of $U_{S}=0.0562$ and fastest growing mode $k_{m}, l_{m}=8,4$; lower right panel is run F2 $(\delta=0.1)$ with an applied shear of $U_{S}=0.037$ and fastest growing mode $k_{m}, l_{m}=9,7$. 
the small initial amplitude) grows substantially to the order of the basic flow. This induced zonal velocity always has a multi-jet structure, and therefore since the basic flow is always constant the emergence of the jets depends on the amplitude ratio between the eddy zonal velocity and the induced zonal velocity. To quantify the

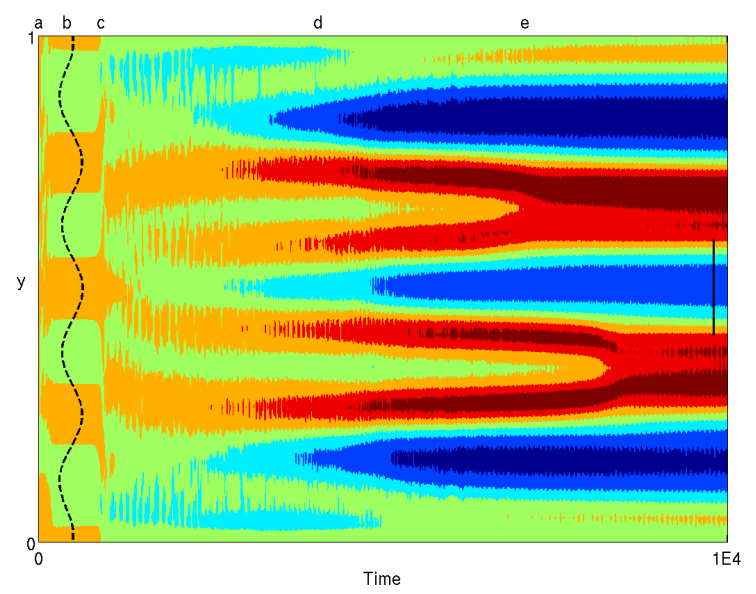

Figure 7.11: The evolution of the induced zonal flow of the top layer $\overline{U_{1}}$ in time. It begins from a weak random field (a) until the fastest growing mode picks up. As this mode grows (b), an induced meridionaly varying flow emerges matching the prediction of the truncated model for $\overline{U_{C}}$ (superimposed by the dashed line), until the nonlinearities become big enough that more modes come in. Then the flow becomes turbulent (c); and the jets become less organized (d), diverging from the initial structure of eq. (7.29). The jet meridional scale increases to the Rhines scale (shown by the solid line on the right), leaving the system with five jets (e). Once it reaches the Rhines scale the induced zonal flow remains stable. A longer time of the same run (S5) can be seen in the bottom panel of Figure 7.12.

strength of the jets we define a parameter $\eta$ for each layer, as the ratio of the mean of the $\overline{U_{n}}$ field to the mean of the eddy field $u^{\prime}$ averaged over time

$$
\eta_{n}=\left(\frac{\left\langle{\overline{U_{n}}}^{2}\right\rangle}{\left\langle u_{n}^{\prime}{ }^{2}\right\rangle}\right)^{\frac{1}{2}} .
$$

Therefore a $\eta>1$ value is a flow dominated by the jets while a $\eta<1$ value is a flow dominated by eddies. The $\eta$ values for both layers in different runs are given in Table 7.1 . 


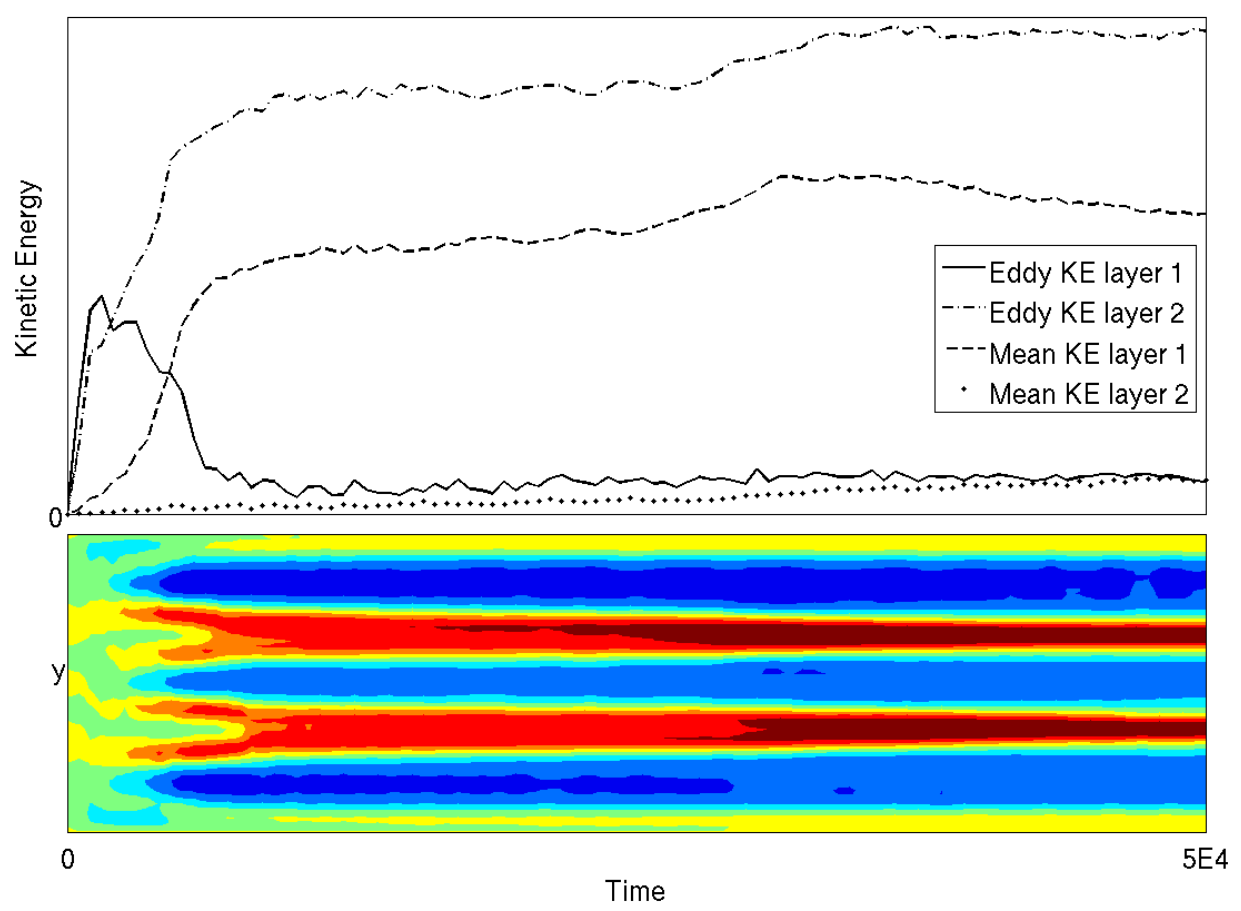

Figure 7.12: The kinetic energy for the induced velocity field $\overline{U_{n}}$ and the eddy field $u_{n}^{\prime}$ for both layers as a function of time (run S5). The bottom panel is the corresponding evolution of $\overline{U_{1}}$ in time across the channel.

Alternatively, it is useful to look at the kinetic energy of the eddies and the mean. Figure 7.12 shows these kinetic energies as a function of time in both layers. Due to the fact that the induced flow is a consequence of eddy interaction, the mean kinetic energy is smaller than the eddy kinetic energy in the initial stages. However once geostrophic turbulence takes over and the energy cascades to larger scales, the kinetic energy is transferred to the mean and the mean zonal flow dominates over the eddy field. In the bottom deep layer however the energy remains in the eddy field, meaning that for this time scale the flow does not become barotropic and the jets are concentrated in the upper layer.

\subsection{Discussion}

In all numerical results shown above, the jets are seen in snapshots of the total velocity field without applying zonal or time averaging. We find that when we use 
such averaging as is often done in studies of jets (c.f. Panetta, 1993) even stronger jets seem to be created and with a bigger $\eta$ value, yet in some of these cases the instantaneous eddy field $u_{n}^{\prime}$ is actually dominant over the $\overline{U_{n}}$ field $(\eta \ll 1)$, and the snapshot plots seem not to resemble jets. This is often the case in the deep layer where the averaged induced zonal velocity $\overline{U_{2}}$ is smaller than $\overline{U_{1}}$ by a factor of $1 / \delta(7.41)$. In the top layer however, whatever the applied shear, there is an induced zonal flow which is typically at least as strong as the eddy field $(\eta>1)$. This can be seen in Table 7.1 where in addition to the standard run showing the relation between the applied shear and the number of jets (Figure 7.9), sensitivity studies to the ratio of $\beta s$ and layer depths are given. We find that the bigger the absolute value of the negative $\beta$, the more jets exist in the top layer (due to the equivalence to weaker shear - Figure 7.1), and the bigger the depth ratio the weaker is the induced zonal velocity in the bottom layer, while the top layer is dominated by jets.

Figure 7.13 shows on the left side panels the instantaneous picture after the system has reached steady state and on the right side panels the corresponding temporally averaged velocity. The upper panels (run S3, $\eta_{1}=3.1$ ) features four westerly jets and three easterly jets in the snapshot plot, and indeed the time mean figure on the right shows the same meridional structure with similar amplitudes. The middle figure (run $\mathrm{S} 1, \eta_{1}=1.87$ ) shows a case with a field showing some jets but within an obvious eddy field, while the time average plot shows distinct jets. The bottom panels show a case where the zonal velocity field is dominated by large eddies (bottom layer of run S13, $\left.\eta_{2}=0.28\right)$ without any jets, yet when averaged the eddies disappear, leaving only the induced zonal velocity which gives an appearance of a strong meridional variation and a jet structure. Therefore we emphasis that in this work we do not need to perform such averaging to the velocity field, and the jets appear in the instantaneous picture. In the observations of the Jovian planets the flow field is established by tracking cloud features, and therefore we expect the instantaneous picture to be most closely related to the data.

The main caveat to keep in mind regarding the model we used here is the assumed baroclinic structure, which although converted from the standard terrestrial case based on estimates of Ingersoll and Pollard (1982), still is quasi-geostrophic, a questionable approximation for deep atmospheres. However, assuming baroclinicity does play a role in the dynamics, and given the Galileo observations of Jupiter that imply that there is an upper non-convective layer (Seiff et al., 1996; Atkinson et al., 

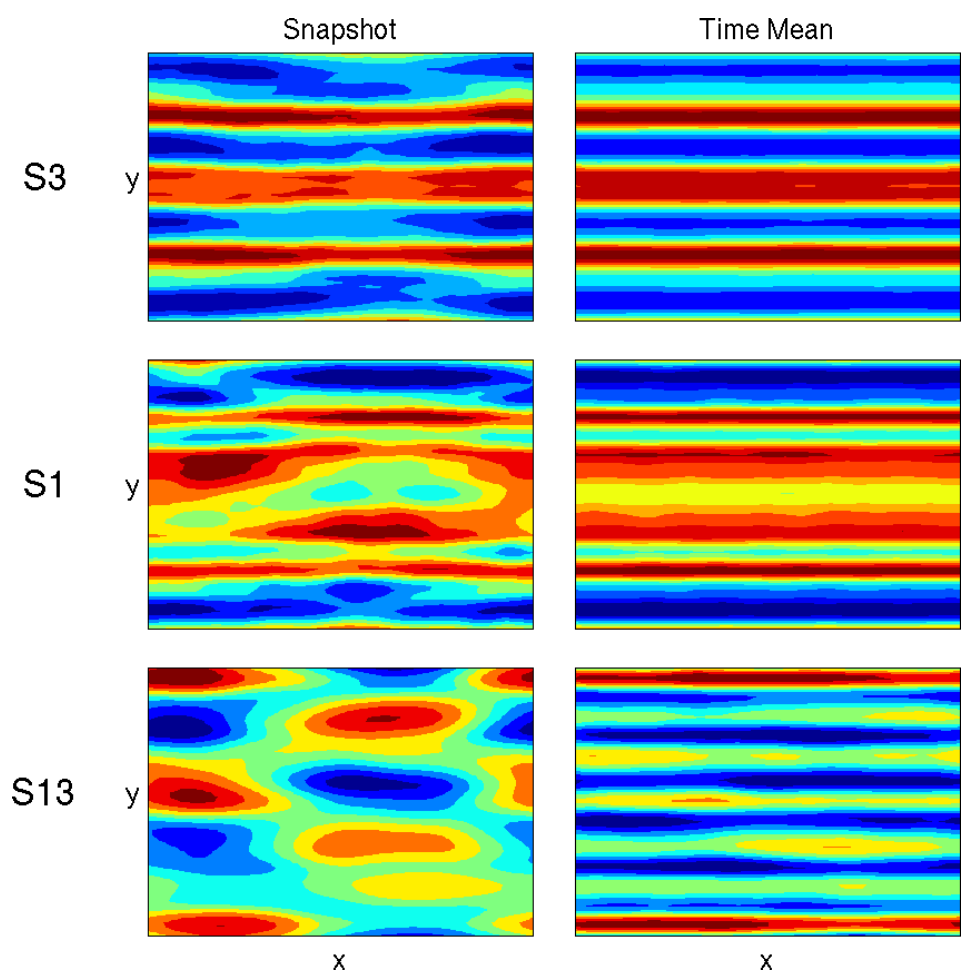

Figure 7.13: Comparison of the instantaneous and time mean steady state total zonal velocity field for different cases. Upper panels show a case (S3) where the time mean represents well the instantaneous field; the middle panels show a case (S1) where the instantaneous field shows some jets but also strong eddies that do not appear in the time mean, and the bottom panels show a case (bottom layer of S13) where the instantaneous picture is totally dominated by big eddies while the averaged picture gives an appearance of a strong meridional variation.

1996) resembling a terrestrial weather layer, then this model may give some insight to the mechanism driving the Jovian jets. Of course there is much more to be desired in terms of observations of the outer planet's atmospheres in order to develop theoretical understanding of the deep columnar structure.

One of the main questions arising from observations on Jupiter and Saturn is that from the data it seems that the barotropic stability condition is violated (Ingersoll and Cuong, 1981; Smith et al., 1982; Stamp and Dowling, 1993), yet the jets seem stable in time. Barotropic instability in a single-beta model, whether one or two layers, is very efficient and eliminates such gradients quickly. Thus, previous barotropic models featuring stable jets had to have this inconsistency with the data. Ingersoll and Pollard (1982) resolve this problem by suggesting an alternative stability crite- 

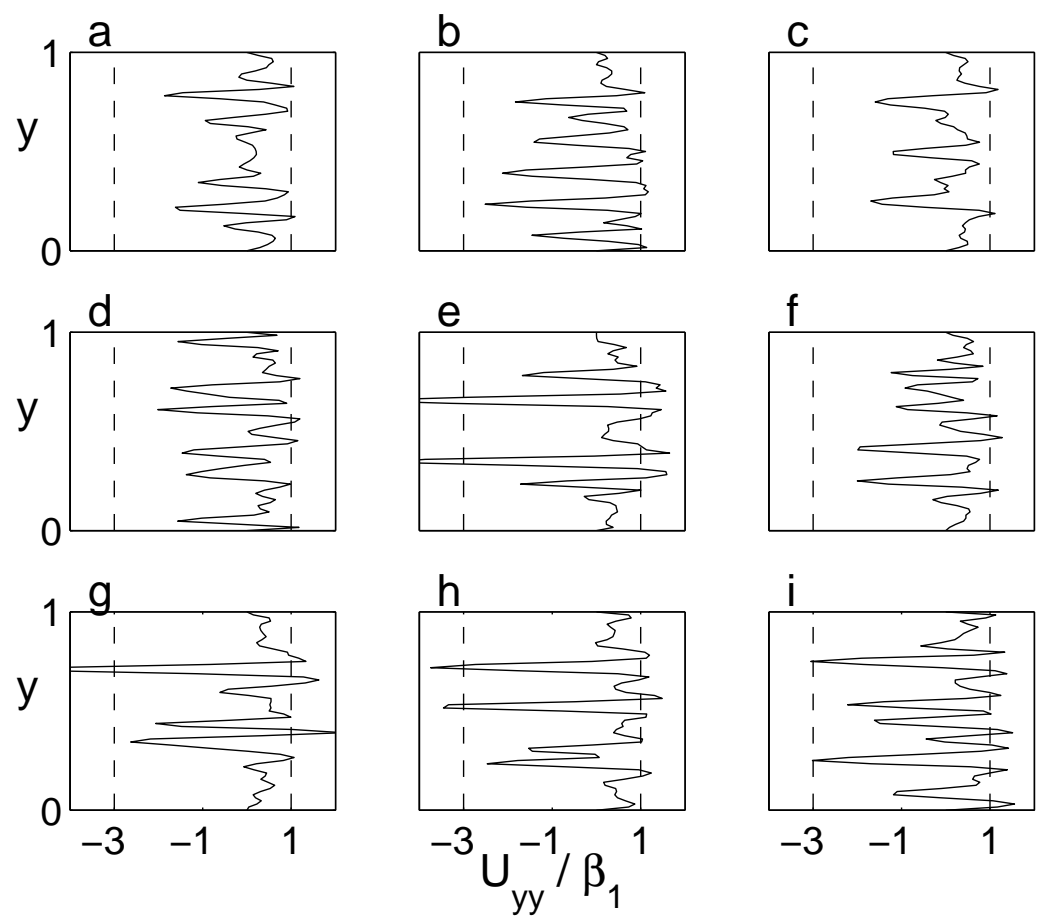

Figure 7.14: The $u_{y y} / \beta_{1}$ curve as function of the width of the channel for some of the runs (a. S1, b. S2, c S3, d. S5, e. S13, f. S4, g. S8, h. S9, i. B2). The dashed line on the right is the standard barotropic stability criterion, and the dashed line on the left is the one suggested by Ingersoll and Pollard (1982). In most cases the curve crosses the barotropic stability curve several times, similar to what is seen in the observations.

rion due to the internal columnar structure. They suggest that in order for the fluid to to have stable jets $\beta$ should be bigger then $-\frac{1}{3} u_{y y}$. Their analysis is consistent with the Voyager data. In our model due to the special geometry used for the bottom convective layer, which results in a scale separation between the scale of the instability and the resulting jets, the barotropic stability condition is still violated (Figure 7.14), and yet the jets are stable in time, due to the continuing exchange of energy between the mean flow and the eddies and the influence of the deep layer on the upper layer flows (in the spirit of Stamp and Dowling (1993), but with jets which are less barotropic). We note that the upper layer $u_{y y}$ appears to approach $\beta_{2}$ on the negative side, indicating again the importance of the coupling between the layers. 


\subsection{Summary}

Traditionally there have been two hypotheses for the existence of the jets observed on the giant planets. One approach (Busse, 1976) assumed that the interior is barotropic and the jets are generated by an internal columnar structure, while the other approach treats the gas planets as a shallow water system (Dowling and Ingersoll, 1989) assuming the deep atmosphere is passive and jets can emerge from eddy interactions (Williams, 1979; Cho and Polvani, 1996). Recent observations (Atkinson et al., 1996; Porco et al., 2003) imply that taking only one of these approaches may be omitting important components of the dynamics. In this chapter we tried to combine these two approaches assuming a baroclinic structure which internally is dominated by columnar structures interacting with an upper layer resembling a regular weather type layer. In addition, we propose that baroclinic instability may provide the energy source, even in the limit of weak vertical shear as suggested by observations.

We show an analytic solution for the generation of jets in the limit of a single mode perturbation interacting with baroclinic zonal flows and then proceed using a pseudo-spectral fully nonlinear numerical model to show that such jets can be generated by baroclinic instability. The analytical solution provides useful insight into the mechanism observed in the numerical results, especially in the way nonlinearity organizes the zonal flow field. The obtained jets are stable on long time scales and are visible in the instantaneous spatial numerical picture without the need for zonal or time averaging. Clearly there is much more to be understood in the dynamics acting in the interior of such gas giants, and such a parametrization of the interior is just a first step. However it does suggest the importance of coupling these two regimes even though they may be governed by very different physical mechanisms. 


\section{Chapter 8}

\section{Conclusion}

\subsection{Thesis Summary}

We look at the results of this work on two different levels. One is the pure fluid dynamical aspect of convection in an anelastic rotating sphere, and the second is the application of the results, and this new GCM, to the dynamics and circulation of the giant planets. We will begin with discussing the first aspect, and then proceed to discuss the second in line with the questions raised in section 1.4 regrading the circulation on the giant planets.

\subsubsection{Convection in a Rotating Anelastic Sphere}

From only a fluid dynamical point of view we have built a new model to examine a problem which has been well studied before. Using anelastic dynamics rather than Boussinesq, the fact that the density anomaly depends on pressure as well as entropy, the general equation of state and the full 3D spherical system, make this treatment unique. We have shown that some of the ideas suggested by linear and Boussinesq theories can be extended into cases of anelastic turbulent convection. In other respects however these solutions are limited.

We begin with looking at the issue of convection in a system where the direction of gravity and rotation are not parallel. This problem has both oceanographic applications (Sheremet, 2004), and is addressed in the planetary literature as well (e.g. Busse et al., 1998). We show that the ratio $\chi=\frac{T a \cdot P r}{R a}$ is an important measure in characterizing the dynamics, and sets a limit between rotationally and gravita- 
tionally dominated convection. This parameter will determine if the convection is aligned with the axis of rotation or not, and therefore whether convection columns are formed. We show that when convection is not aligned with the axis of rotation, superrotation will not develop, and in fact for such cases, the equatorial velocity will be retrograde. Superrotation forms only when convection columns align parallel to the axes of rotation and are tilted in the direction of the shear. We find that this is a robust result as long as $\chi>1$, due to convection driving the fluid to being close to isentropic. The number of columns and amplitude of the shear depends on the values of the nondimensional numbers.

The issue of the tilting or spiraling of the columns was shown previously by studies such as Zhang (1992). These cases however were either linear or weakly nonlinear so that both cyclonic and anticyclonic cells formed. We find that this state can not be sustained when the shear becomes strong, and therefore we find it only when velocities are small during spin-up. When the shear exceeds a critical value only the rotating cells that are in the direction of the shear are sustained. The circulation cells before and after this transition are tilted and provide therefore an angular momentum flux outward. The tilt or spiraling of the columns is associated with the variation in planetary vorticity in the direction perpendicular to the rotation axis. However there is still a single and positive phase velocity which sets propagation of the modes. While the perturbation is growing, the angular momentum flux is balanced by the growing mean zonal velocity. The shear is created by the prograde surface flow on the exterior and retrograde surface flow in the interior. Once the shear has become large enough and the circulation undergoes a transition to having only cyclonic cells the balance is between the upgradient momentum fluxes and the viscous fluxes. Superrotation is maintained near the equator and is stable, while in the interior there is a subrotating flow.

The strength of the subrotation depends on the level of compressibility of the fluid. In a Boussinesq fluid the strength of the westward subrotation in the interior would be comparable to the eastward superrotation. However when the density in the interior is larger, the subrotating flow will be weaker than the superrotation due to a baroclinic contribution to vorticity which is associated with the compressibility of the fluid (section 4.3). Anelastic effects are therefore strongest along the outside edge of the sphere, where compressibility is greatest. We find that this radial shear associated with the compressibility varies also in latitude. 
Although superrotation has been shown in several 3D Boussinesq studies, these have not demonstrated the link to the linear theories and have not shown explicitly the mechanism for the superrotation in a 3D system. In some of the other models this circulation appears only when averaging in time. To the best of our knowledge this is the first model which shows the convective columns explicitly in the $3 \mathrm{D}$ flow, the eastward propagation of these columns, the shear within the column in the direction of the rotation axis and the transition to only columns rotating in the direction of the shear. In chapters 4 and 5 we use simpler models to understand the physics governing these processes, and give approximate analytic expressions to their dependence on the properties of the fluid.

\subsubsection{Application to the Atmospheres of the Giant Planets}

The second aspect of this work is the application of these results to the dynamics of the giant planets. In this respect, our general circulation model is an improvement over previous models due to the including of compressible dynamics, more realistic thermodynamics and a forcing which is not confined to the domain boundaries. Beyond the improved physics an important advantage of this new model is that it is now part of the MITgcm standard package, and can be downloaded and used by anyone. One of the problems with comparing numerical results is the limited accessibility to other models and specific configurations which could not be cross examined. The open code philosophy of the MITgcm and the available manual and help resources, make this model easily accessible.

In section 1.4 we have raised several questions regarding the circulation on the giant planets. We come back to these in light of our study.

Both Jupiter and Saturn are dominated with a strong superrotating equatorial flow (Figure 1.1). We have shown that angular momentum eddy fluxes associated with convection in a rotating system can drive superrotation with velocities similar to the velocities on the giant planets. The fluid velocities in our model do depend on the Rayleigh and Ekman numbers; however the mechanism for superrotation does not depend on the nondimensional numbers (see discussion in 5). We show in section 4.7 that although the velocities depend on the nondimensional numbers the velocity profile remains consistent for a range of Rayleigh numbers (Figure 4.16). Another result which our model configuration is sensitive to is the latitudinal extent of the 
superrotating jet. For our standard type simulation (with an inner boundary at about half the radius of the planet) the jet is wider than the observation on Jupiter (close Saturn's superrotation). We show however that for a relatively shallow domain the jet width is sensitive to the location of the bottom boundary. Due to the uncertainty in the dynamics of the interior, this suggests a link between the width of the superrotation and the depth of the jet. This is consistent also with the weaker interior circulation due to anelastic effects. We find that for more turbulent flows (higher Rayleigh number, lower Ekman number) the equatorial jet is narrower and stronger.

We focused our work on Jupiter parameters but much of our results should apply to Saturn as well and to a lesser extent to Uranus and Neptune which probably have a much shallower dynamical region. This brings the question of why are the equatorial winds on the gas giants superrotating, while on the ice giants they are subrotating? We described above the mechanism for superrotation on the gas planets. We find that the only criterion that the fluid has to obey in order to get superrotation is that the parameter we defined as $\chi=\frac{T a \cdot P r}{R a}$ is greater than one. Descriptively that means that the fluid is dominated by rotation and not by convection. We show that for the parameters of Jupiter and Saturn this indeed is the case. This raises a question about the ice-giants since while rotation on them is not even half as slow, convection is at least an order of magnitude weaker than on the gas giants, implying therefore that rotation is even more dominant. However, since the $\chi$ parameter also depends on the domain depth, the $\chi$ parameter may still be less than one for the ice-giants. Particularly, given the gravitational acceleration on the ice-giants, if the depth of the relevant fluid region is 30 times smaller, this would balance a flux which is an order of magnitude smaller. Then the shallower circulation may bring the planets to a regime of equatorial subrotation.

Another key question is how deep are the zonal winds. Thermal wind estimates and the Galileo probe observations have provided some data (section 1.2) yet, also much uncertainty. The main advantage of this model compared to the previous Boussinesq models is that it is closer to a realistic dynamic and thermodynamic representation of the interior. However if the fluid is barotropic and has small Rossby and Ekman numbers we would still expect the Taylor-Proudman theorem to hold whether the fluid is Boussinesq or anelastic. We find though, that the convection does homogenize the entropy so that baroclinic terms are still important in the vorticity balance. Particularly the strong variation in density in the upper levels gives a big 
baroclinic contribution due to compressibility, which results in a zonal velocity shear within the convection columns. Consequently the interior zonal velocities are weaker. This result is different than previous suggestions, which either assumed a deep source for the jets with strong interior velocities or a shallow driving force and weak interior velocities. Therefore solely due to compressible effects we expect to find vertical shear resulting in weaker interior velocities.

Recent observations have identified waves embedded within the equatorial superrotation. We find waves in our simulations which are a surface manifestation of the convection columns and are therefore embedded within the superrotation. It is difficult to be sure the observed waves have the same dynamics, since the observed waves have a wavelength which is $\sim \frac{1}{3}$ of the finest grid resolution in our simulations. However the spacial resemblance with crests centered at the equator that are curved eastward, the phase speed which is about equal to the mean zonal velocity, and the fact that for more turbulent flow we find waves which are narrower and with a higher wave number brings us to hypothesize that the waves that we see on the planet are related to fine structure convection columns from within Jupiter's interior. If indeed this is the case, then based on the latitudinal extent of the waves $\left(10^{\circ}\right)$ the columns will penetrate no more than $1 \%$ into the interior of the planet.

Another important question is why is there a nearly latitudinally uniform thermal emission on the gas giants. Since solar forcing is stronger at the equator and lower at the poles (not considering seasonal effects), there must be meridional heat transport in the poleward direction. Due to the strong zonal dominance at the cloud level it is unlikely that this is a shallow process. To address this question quantitatively we must have solar forcing in our model (section 8.2.1). However the alignment of the convective heat fluxes along the direction of the axis of rotation (Figure 4.8), leads us to hypothesize that the relative heating of the pole may be associated with transport of the interior heat poleward and not the meridional redistribution of the solar heat.

Finally we come to the question of the jet stability and multiple zonal jets. The convection model typically produces an eastward jet at the equator, two westward jets in midlatitude and a region dominated by eddies at high latitudes. It is probable that the high meridional wind structure is associated with turbulent processes that our convection model can not resolve (although we do find multiple jet structures which are not steady over time - section 6.2). Particularly important may be shear processes in the upper stably stratified levels. In our two layer model we provide 
a representation of the deep interior, and find that multiple baroclinic zonal jets emerge from decaying quasigeostrophic turbulence. The energy of these jets comes from baroclinic instability. These jets are stable due to eddy mean flow interactions, similar to the processes we have seen stabilizing the superrotating jet in the convection model. We suggest therefore that even weak baroclinic instability (due to the weak meridional temperature gradient) can provide an energy source for jets. This model points to the possibility that the deep mechanism that we described previously is the underlying basic structure of the winds, and the shallow shear processes overlay this deep induced flow.

\subsection{Future Work}

\subsubsection{Solar Forcing}

One of the original goals of this work was to study the interaction between the convective driven flow and the circulation driven by solar forced meridional temperature gradients. We have experimented with cases where the interior is driven by the regular convection, while the top is forced by a latitudinally varying entropy gradient, so that the upper levels are gravitationally stable. The problem of having a model which can both treat the convection and resolve shear instabilities turns out to be very computationally demanding. The problem rises since the viscosities which must be used for the convection problem make the Reynolds number too small for shear instabilities. We have experimented with different ways of resolving this problem such as separating the horizontal and vertical values of the physical parameters, and increasing the resolution of the simulation. We have had only partial success in doing this and this is left for future research. An inherent problem is that in a convective system the deformation radius by definition is non-existent to extremely small, and therefore resolving this scale is not possible. The existence of a stably stratified layer on top is therefore essential.

We will show however some very initial results of the 2D system. In Figure 8.1 we show two cases where one is driven only by an exterior temperature gradient with no internal convection (left panel), and the second has both internal and external driving. The external forcing creates a large Hadley type cell which in the presence of convection is broken by the convective structures which as we have shown in Figure 

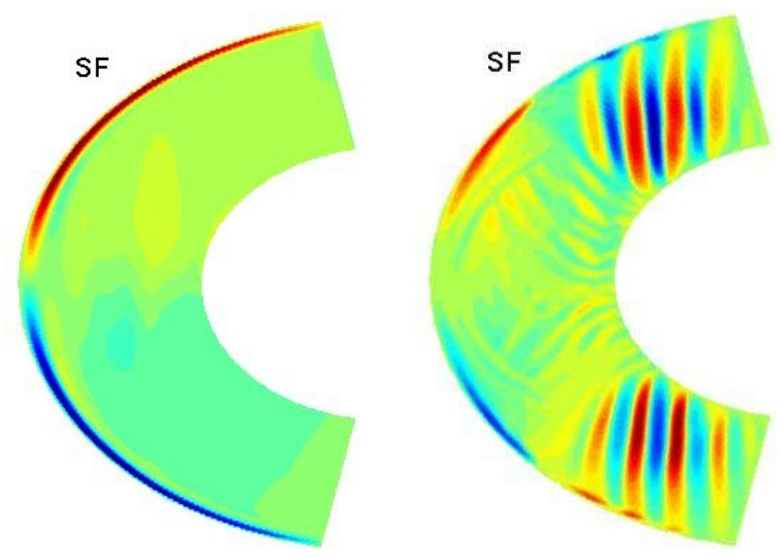

Figure 8.1: The meridional streamfunction for cases driven by only an exterior temperature gradient (left) and both internal convection and a similar exterior forcing (right).

3.4 when comparing the 2D and 3D results, and are stronger at high latitudes in 2D. Since these are only 2D cases there is no equatorial superrotation. When repeating these experiments in $3 \mathrm{D}$ for the parameter regime of the convection model we find similar large Hadley type structures with an associated zonal velocity which increases away from the equator. As mentioned the interaction of the exterior and interior forced system in $3 \mathrm{D}$ is left for future work.

\subsubsection{Gravitational Moments}

Since the giant planets have short rotation periods and they are essentially fluid objects, the planets bulge out at the equator in response to the centrifugal force (see Table 1.1). Since we have made the approximation of using a sphere and not an oblate sphere we do not expect that when calculating the low order gravitational moments from the density field they would match the planets low order gravitational moments. However the higher order moments are less dependent on the oblateness of the planet. Hubbard (1999) has suggested that precise measurements of the high order gravitational moments can give information on the deep wind structure of the planet. He showed that two extrema cases, one where the whole planet rotates as a solid body and the other where there is rotation along concentric cylinders as suggested by Busse (1976) differ considerably beyond the tenth moment. In this section we look at sensitivity of the gravitational moments calculated for our model for two cases, an 
anelastic and Boussinesq case.

The gravitational potential outside a planet satisfies Laplace's equation and in spherical coordinates (but limiting to an axisymmetric solution) has a solution of the form

$$
V=\sum_{n}^{\infty}\left(A_{n} r^{n}+B_{n} r^{-(n+1)}\right) P_{n}(\sin \theta)
$$

where $P_{n}$ are Legendre polynomials . Assuming the potential will tend to zero as $r$ goes to infinity allows taking $A_{n}=0$. Then taking the gradient of the potential we can find the gravitational acceleration as a function of the radius

$$
g(r, \theta)=-\frac{G M}{r^{2}}\left(1-\sum_{n=2}^{\infty} J_{n}\left(\frac{R}{r}\right)^{n} P_{n}(\sin \theta)\right)
$$

where $G$ is the Cavendish constant and $M$ is the mass. We can then compute $J_{n}$ by calculating the appropriate moments of the density distribution. Thus given the density distribution from our model we can calculate the gravitational moments. In Figure 8.2 we compare the moments resulting from our model for anelastic and Boussinesq 3D cases to the ones estimated by Hubbard (1999). The green and black dots are the moments for a model where the planet is rotating like a solid body and where the planet is rotating along concentric cylinders respectively as calculated by Hubbard (1999). The diamonds are the observed values for $J_{2}, J_{4}$ and $J_{6}$ as measured using Pioneer and Voyager data (Campbell and Synnott, 1985). As can be seen from the green and black curves beyond $n=10$ the two scenarios tested by Hubbard diverge significantly. The red points are the moments calculated using the density anomalies from the anelastic model, and blue points are the moments calculated using the density anomalies from the Boussinesq model. Since our model is a symmetric sphere the low order moments which are dominated by the oblateness of the planet do not appear in our calculations. The $J_{8}$ moment however matches Hubbard's model for both the anelastic and Boussinesq cases.

The significance of the this result is still to be determined. We have tested the sensitivity of the gravitational moment results and found so far that for experiments Ra1 and Ra2 (Table 6.1) the moments are affected somewhat by the choice of the Rayleigh number but we could possibly estimate this effect by a limiting to Rayleigh 


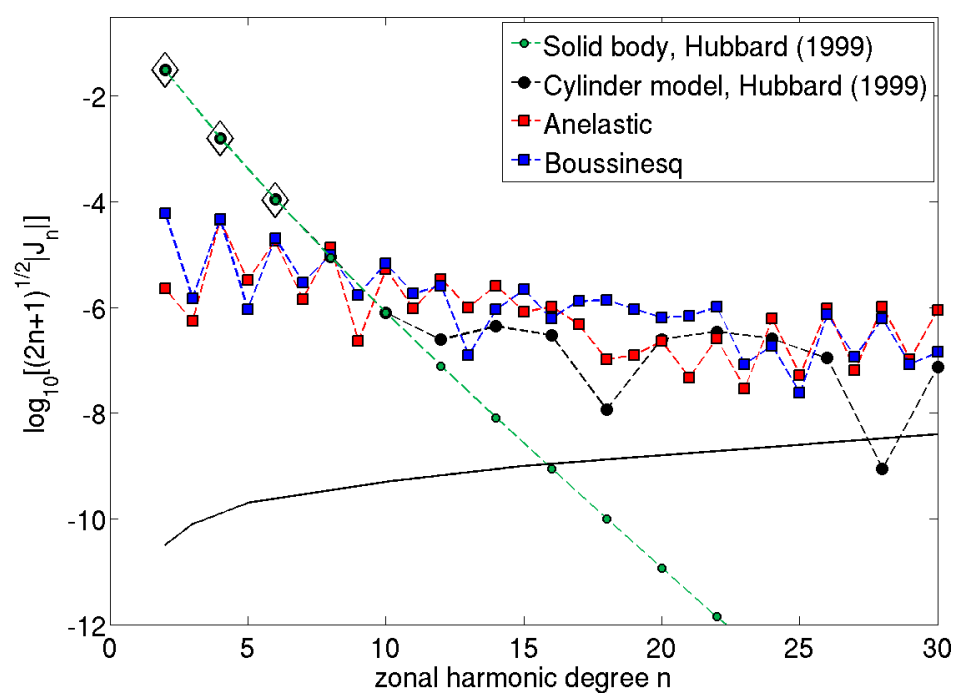

Figure 8.2: Gravitational moments for different interior velocity structures. The green dots are the moments for a model where the planet is rotating like a solid body and the black dots are the moments where the planet is rotating along concentric cylinders. In both cases the data is courtesy of Bill Hubbard. The diamonds are the observed values (Campbell and Synnott, 1985). The red points are the moments using our anelastic model, and blue points are the moments using the Boussinesq model. The solid line is the detectable limit of JUNO.

numbers that have velocities on the order of the ones on the planet. A particular question that has been raised is a discrepancy between the theoretical and the observed $J_{4}$ moments. One possibility is that this discrepancy is due to density fluctuations due to the velocity fields (which are not taken into account in the theoretical calculations). Studying the effect of density fluctuations in our model on $J_{4}$ might help in addressing the importance of the circulation related density fluctuations. This study is in very preliminary stages, and is brought as an addendum to the thesis, highlighting a possible particular implementation of our model. This becomes now particularly relevant due to the upcoming JUNO mission to Jupiter, which will measure the high order gravity moments in order to try and solve the question of how deep are the zonal winds. The mission is scheduled to be launched in 2011 and reach Jupiter in 2017. The solid black line in Figure 8.2 is the detectable limit of JUNO. The model might be able to distinguish between the gravitational signature of different velocity structures and address the discrepancy in $J_{4}$. 


\section{Appendix A}

\section{Applying the Deep Anelastic System to the MITgcm}

In this appendix we discuss some of the technical details associated with adapting the MITgcm to giant planets. Due to the nonhydrostatic capability of the ocean-MITgcm, we have chose to use it over the atmospheric version. The atmospheric version would allow to treat more easily the compressibility effects, but addapting the nonhyrostatic version to pressure coordinates adds other difficulties (see further discusion in section 2.1). Therefore we adopt the anelastic approximation and use the ocean-MITgcm.

\section{Extending the model to a full sphere}

The MITgcm has been designed for for calculations on a thin spherical shell, and therefore did not allow a vertical variation of the grid size (the zonal grid does vary as a function of latitude). The depth of the ocean is typically less than $0.1 \%$ of the radius of the planet, and thus allowing such an approximation. When extending the model to a full sphere, horizontal grid size must change as a function of depth and maintain all vertical fluxes. We have applied this by defining a geometrical factor based on the spherical geometry, which is set in a vector that multiplies all zonal and meridional grid spacings $(d x$ and $d y$ ). We have verified this modification by comparing results for for different planet radii, and comparing fluxes and geometrical factors in simple test cases. 


\section{Applying the anelastic dynamics}

Similarly to redefining the grid spacings we have defined a vector that multiplies the mean density by a factor which varies as a function of depth. This factor is calculated from the mean density which is set by the adiabatic reference state and the equation of state (see below). Having the model written in flux form allowed inserting these factors directly into the divergence. The MITgcm solves first for the hydrostatic pressure (2D solver) and then for the nonhydrostatic part (3D solver). In both the Laplace type equation for pressure is solved in an iterative process. Making the density a function of depth requires special care since the vertical components have an additional terms (3D solver). We have checked consistency in the Boussinesq limit (although the Boussinesq limit is simpler not only because the independence of $\bar{\rho}$ but also because the density fluctuations from the equation of state are not a function of pressure), and verified fluxes consistantancy.

\section{Implementing interior and exterior forcing}

We have discussed the interior forcing by a continuous forcing profile in section 2.5. This is implemented by using the mean temperature profile $\bar{T}$ (set by the reference state) to calculate the forcing in (2.48). We then constrain the heating so that, when integrated over the whole volume, the total forcing will be zero, and thus no net heating is added from the system. We do so by integrating this profile weighted by the vertical grid spacing and the mean density. This basically shifts the cooling profile of the planet so that the interior is heating and the exterior is cooling. The heating for every vertical level is added to the external forcing routine of the MITgcm as entropy per unit time. The second, simpler, profile we have used and discussed in sections 3.1 .2 and 6.4 is simply applying a heating rate to the bottom boundary. We balance the heating by relaxing the top few layers to a reference temperature which we can deduce from the observed values (Seiff et al., 1997). The solar heating is applied as a meridionally varying heating of the top grid levels. We apply this in the same way as the bottom heat flux with a Newtonian relaxation on top (section 8.2.1). 


\section{Setting the reference state}

The reference state of the model is first set by setting the vertical grid spacing. We have found that the most numerical stable configuration is to set a constant factor in which the mean pressure grows as a function of depth down to a depth beyond which the vertical grid spacing itself is constant. Using a geometric series in mean pressure all the way down will require a very high resolution at the top of the atmosphere, in order to get reasonable representation of the interior. Using constant grid spacing throughout is not necessary because most of the scale heights are on top. Another possibility which can be attempted is setting a constant number of grid points per scale height, but this might have an unnecessary resolution in the interior. Once the vertical pressure spacing is set we use the tables of Guillot and Morel (1995), to determine the relative depth of each mean pressure level. This allows calculating the level depths $(d z)$, which are the input of the ocean MITgcm.

As discussed in section 2.2.1 we assume an adiabatic reference state. We use the data from the Galileo probe (Seiff et al., 1997) to set the constant reference entropy level of the adiabat. Then using the SCVH EOS (Saumon et al., 1995) we can find the temperature and density vertical profiles of this adiabatic reference state. We

find that this profile based on the entropy value found by the Galileo probe (which is close to being constant, but goes down only to 24 bars) matches well the deep temperature and density profiles of Guillot and Morel (1995), (Figure 2.5). Once the density, entropy, pressure and temperature vertical profiles are set we can calculate the local density dependence on entropy along isobars, and the density dependence on pressure along the adiabat, which will be the coefficients for the equation of state.

\section{Implementing the SCVH equation of state}

For the ocean-MITgcm the EOS is given as a polynomial, where density is a function of potential temperature and salinity. We take advantage of this framework (poly3), and set the density as a function of entropy and pressure. We define a revised entropy variable so that entropy could be written in terms of potential temperature so that this converted entropy is defined as

$$
s^{\prime}=\bar{T}(1) e^{\frac{\sigma^{\prime}}{C_{p}}}
$$


where $\sigma^{\prime}$ is the original entropy in entropy units, and $C_{p}$ is the isobaric specific heat for an ideal gas (we show it does not vary considerably even in the interior). Therefore the entropy has an arbitrary constant (the reference level) in its value but this does not effect the dynamics set by equations (2.8,2.9,2.10,2.7,2.28 and 2.47). For every level in our vertical grid we then match a polynomial to the variation of density as function of entropy along the mean isobar. This gives us a vector with the leading order coefficient of the polynomial (for each vertical level) which we then use for the dependence of density on entropy in the EOS. The second set of coefficients (dependence of density on pressure along an adiabat) is simpler since the reference state is adiabatic and therefore these coefficients can be deduced from the mean fields. We calculate the vertical gradient of density in respect to pressure for every vertical level and obtain a second set of coefficients. Then using the framework of the poly3 EOS we determine the full density as a sum of the reference mean density for each vertical level, and the entropy and pressure anomalies weighted by the coefficients described above so that

$$
\rho(s, p)=\bar{\rho}+\left(\frac{\partial \rho}{\partial s}\right)_{\bar{p}} s^{\prime}+\left(\frac{\partial \rho}{\partial p}\right)_{\bar{s}} p^{\prime} .
$$

For a Boussinesq system the density anomalies will not be a function of pressure and therefore we set the second set of coefficients to zero and the last term vanishes. This reduces computation time by almost an order of magnitude compared to the anelastic case, since the pressure fluctuation is obtained from the previous time step, and therefore requiring a small time step (typically 5seconds for the $1^{\circ}$ resolution runs). We have shows in section 4.3.2 the necessity of including the pressure anomalies in the EOS for the anelastic case. We also use the SCVH EOS to calculate the density and temperature mean profiles along the adiabat we have set for the reference state as described above.

\section{Implementing the variation in gravity}

In the terrestrial spherical shell models, since the model occupy only a small fracture of the planetary radius, the gravitational acceleration is taken as a constant. Here, in the deep model we can not make this approximation, and we calculate the gravitational acceleration separately for every vertical grid point. This is calculated by integrating the mean density (2.4) from the interior to the vertical level where $g$ is calculated. 
We calibrate the interior values so that the gravitational acceleration at the surface matches that measured on Jupiter. The model is then given this vector similar to the way we implement the vertical variation in grid size and density. 


\section{Appendix B}

\section{Nonlinear Truncated Solutions for the Two-Beta Model}

\section{B.1 The Derivation of the Truncated Model}

In this Appendix we derive the nonlinear solution for the truncated model. We begin by rewriting the barotropic and baroclinic perturbation equations using $Q_{T}$ and $Q_{C}$ as defined by (7.18 and 7.19).

$$
\begin{aligned}
\frac{\partial}{\partial t} q_{T}+U_{C} \frac{\partial}{\partial x} q_{C}+Q_{T} \frac{\partial}{\partial x} \phi_{T}+Q_{C} \frac{\partial}{\partial x} \phi_{C} & \\
+J\left(\phi_{T}, q_{T}\right)+J\left(\phi_{C}, q_{C}\right) & =0 \\
\frac{\partial}{\partial t} q_{C}+U_{C}\left(\frac{\partial}{\partial x} q_{T}+\xi \frac{\partial}{\partial x} q_{C}\right)+Q_{T} \frac{\partial}{\partial x} \phi_{C} & \\
+Q_{C}\left(\frac{\partial}{\partial x} \phi_{T}+\xi \frac{\partial}{\partial x} \phi_{C}\right)+J\left(\phi_{T}+\xi \phi_{C}, q_{C}\right)+J\left(\phi_{C}, q_{T}\right) & =0
\end{aligned}
$$

Expressing the perturbation potential vorticity as a single perturbation wave (7.22, 7.23) and using the inversion relations $(7.24,7.25)$ for the streamfunctions, we find that the Jacobians in the barotropic equations vanish while the ones in the baroclinic equations give an expression of the form

$$
\overline{q_{C}}=\overline{q_{C}^{\prime}}(t) \sin (2 l y)
$$


as given by (7.26). Using the inversion relation (7.28) and the boundary condition (7.8) gives an expression for the correction to the basic streamfunction given by

$$
\begin{aligned}
\overline{\phi_{C}}= & \frac{-\overline{q_{C}^{\prime}}(t)}{4 l^{2}+F_{1}+F_{2}} \\
& \cdot\left[\sin (2 l y)-\frac{\sinh \left[\sqrt{F_{1}+F_{2}}\left(y-\frac{1}{2}\right)\right]}{\sqrt{F_{1}+F_{2}} \cosh \left(\frac{\sqrt{F_{1}+F_{2}}}{2}\right)}\right]
\end{aligned}
$$

which when differentiated gives a correction to the mean flow as given in (7.29). Now we rewrite the barotropic and baroclinic perturbation equations (B.1,B.2) using (7.22,7.23 and B.3). For clarity we note that the baroclinic basic zonal flow now has the form

$$
U_{C}=U_{0}+\overline{U_{C}} \equiv U_{0}+\overline{q_{C}^{\prime}}(t) f_{u}(y)
$$

where $f_{u}$ is defined by (7.29). Then (B.1,B.2) become

$$
\begin{gathered}
\left.\sin (l y) \frac{\partial}{\partial t} q_{T}^{\prime}+i k \sin (l y)\right)\left[\left(U_{0}+\overline{q_{C}^{\prime}} f_{u}\right) q_{C}^{\prime}-Q_{T} \frac{q_{T}^{\prime}}{\kappa^{2}}-\right. \\
\left.\left(Q_{C}+2 l \overline{q_{C}^{\prime}} \cos (2 l y)\right) \frac{q_{C}^{\prime}}{\kappa^{2}+F_{1}+F_{2}}\right]=0 \\
\frac{\partial}{\partial t}\left(\sin (l y) q_{C}^{\prime}+\sin (2 l y) q_{C}^{\prime}\right)+ \\
i k \sin (l y)\left[\left(U_{0}+\overline{q_{C}^{\prime}} f_{u}\right)\left(q_{C}^{\prime}+\xi q_{C}^{\prime}\right)-Q_{T} \frac{q_{C}^{\prime}}{\kappa^{2}+F_{1}+F_{2}}-\right. \\
\left.\left(Q_{C}+2 l \overline{q_{C}^{\prime}} \cos (2 l y)\right)\left(\frac{q_{T}^{\prime}}{\kappa^{2}}+\frac{\xi q_{C}^{\prime}}{\kappa^{2}+F_{1}+F_{2}}\right)\right] \\
\frac{i k l \sin (2 l y)\left(F_{1}+F_{2}\right)}{\kappa^{2}\left(\kappa^{2}+F_{1}+F_{2}\right)}\left[q_{C}^{\prime} q_{T}^{\prime *}-q_{C}^{*} q_{T}^{\prime}\right]=0
\end{gathered}
$$


Multiplying by $\sin (l y)$, integrating over the channel and normalizing by $\int \sin (l y)^{2} d y$ gives

$$
\begin{array}{r}
\frac{\partial}{\partial t} q_{T}^{\prime}+i k\left[\left(U_{0}+\overline{q_{C}^{\prime}} I_{u}\right) q_{C}^{\prime}-Q_{T} \frac{q_{T}^{\prime}}{a^{2}}-\right. \\
\left.\left(Q_{C}-l \overline{q_{C}^{\prime}}\right) \frac{q_{C}^{\prime}}{\kappa^{2}+F_{1}+F_{2}}\right]=0 \\
\frac{\partial}{\partial t} q_{C}^{\prime}+i k\left[\left(U_{0}+\overline{q_{C}^{\prime}} I_{u}\right)\left(q_{C}^{\prime}+\xi q_{C}^{\prime}\right)-Q_{T} \frac{q_{C}^{\prime}}{\kappa^{2}+F_{1}+F_{2}}\right. \\
\left.\left(Q_{C}-l \overline{q_{C}^{\prime}}\right)\left(\frac{q_{T}^{\prime}}{\kappa^{2}}+\frac{\xi q_{C}^{\prime}}{\kappa^{2}+F_{1}+F_{2}}\right)\right]=0
\end{array}
$$

where the integral $I_{u}$ is defined as

$$
\begin{aligned}
I_{u}= & \frac{-l}{4 l^{2}+F_{1}+F_{2}} \\
& \cdot\left[1+\frac{16 l^{3} \tanh \left(\frac{\pi \sqrt{F_{1}+F_{2}}}{2 l}\right)}{\pi \sqrt{F_{1}+F_{2}}\left(4 l^{2}+F_{1}+F_{2}\right)}\right]
\end{aligned}
$$

while projecting by $\sin (2 l y)$ gives an equation for the baroclinic mean correction

$$
\frac{\partial}{\partial t} \overline{q_{C}^{\prime}}+\frac{i k l\left(F_{1}+F_{2}\right)}{\kappa^{2}\left(\kappa^{2}+F_{1}+F_{2}\right)}\left[q_{C}^{\prime} q_{T}^{*}-q_{C}^{\prime *} q_{T}^{\prime}\right]=0
$$

Equations (B.8, B.9 and B.11) define the system which we can solve for $q_{T}^{\prime}, q_{C}^{\prime}$ and $\overline{q_{C}^{\prime}}$. We rewrite the equation for the perturbation (B.8) and (B.9) in the form

$$
\frac{\partial}{\partial t} \mathbf{q}+i k \mathbf{L q}+i k \overline{q_{C}^{\prime}} \mathbf{N q}=0
$$

where $\mathbf{q}=\left(\begin{array}{c}q_{T}^{\prime} \\ q_{C}^{\prime}\end{array}\right)$ and the operators are

$$
\begin{aligned}
\mathbf{L} & =\left(\begin{array}{cc}
-\frac{Q_{T}}{\kappa^{2}} & U_{0}-\frac{Q_{C}}{\kappa^{2}+F_{1}+F_{2}} \\
U_{0}-\frac{Q_{C}}{\kappa^{2}} & \xi U_{0}-\frac{Q_{T}+\xi Q_{C}}{\kappa^{2}+F_{1}+F_{2}}
\end{array}\right) \\
\mathbf{N} & =\left(\begin{array}{cc}
0 & I_{u}+\frac{l}{\kappa^{2}+F_{1}+F_{2}} \\
I_{u}+\frac{l}{\kappa^{2}} & \xi I_{u}+\frac{\xi l}{\kappa^{2}+F_{1}+F_{2}}
\end{array}\right)
\end{aligned}
$$


The limit where $\mathbf{N}=0$ gives back the linear problem.

\section{B.2 The Weakly Nonlinear Limit to the Truncated Model}

In this section we give the full derivation for the analytic expressions for the components of the Landau-Gintzburg equation for the amplitude of the weakly nonlinear perturbation given in (7.40). This limit where the shear is taken to be just slightly supercritical is similar to the weakly supercritical theory of Pedlosky (1970), only for a more general case of different layer depths and a variable $\beta$. In the linear problem we noted that (7.33) the linear growth rate is proportional to $\Delta^{\frac{1}{2}}$, where $\Delta$ was a small increase to the critical shear. Therefore we can define a slow time scale (so far we have treated the truncated nonlinear problem without requiring it to be weakly supercritical or defining a slow time scale) thus

$$
\frac{\partial}{\partial t} \rightarrow \frac{\partial}{\partial t}+\Delta^{\frac{1}{2}} \frac{\partial}{\partial T}
$$

and the slow time expansion sets the operator

$$
\mathbf{L}=\mathbf{L}_{0}+\Delta \mathbf{L}_{2}
$$

where $\mathbf{L}_{0}$ and $\mathbf{L}_{2}$ are

$$
\begin{aligned}
\mathbf{L}_{0} & =\left(\begin{array}{cc}
-\frac{Q_{T}}{\kappa^{2}} & U_{0}-\frac{Q_{C}}{\kappa^{2}+F_{1}+F_{2}} \\
U_{0}-\frac{Q_{C}}{\kappa^{2}} & \xi U_{0}-\frac{Q_{T}+\xi Q_{C}}{\kappa^{2}+F_{1}+F_{2}}
\end{array}\right) \\
\mathbf{L}_{2}= & \left(\begin{array}{cc}
-\frac{\sqrt{\delta} F_{1}}{\kappa^{2}}+\frac{F_{2}}{\sqrt{\delta} \kappa^{2}} & 1-\frac{F_{1}+F_{2}}{\kappa^{2}+F_{1}+F_{2}} \\
1-\frac{F_{1}+F_{2}}{\kappa^{2}} & \xi-\frac{\sqrt{\delta} F_{1}}{\kappa^{2}+F_{1}+F_{2}}+\frac{F_{2}}{\sqrt{\delta}\left(\kappa^{2}+F_{1}+F_{2}\right)}+\frac{\xi\left[1-\left(F_{1}+F_{2}\right)\right]}{\kappa^{2}+F_{1}+F_{2}}
\end{array}\right)
\end{aligned}
$$

for $U_{0} \rightarrow U_{0}+\Delta$. We expand

$$
q=e^{-i k C_{0} t}\left[q_{0}+\Delta^{\frac{1}{2}} q_{1}+\Delta q_{2}+O\left(\Delta^{\frac{3}{2}}\right)\right]
$$

and we assume the system is weakly nonlinear thus $\mathbf{N}$ will be $O(\Delta)$. By the choice of $c_{0}$ as the growth rate, $\mathbf{q}_{0}$ does not depend on $t . q 1$ and $q 2$ may depend on $t$ but when expanding, the solvability condition implies that all have the same $t$ dependents 
(they depend differently on the slow time scale $T$ ). Then expanding (B.12) in powers of $\Delta$, gives

$$
\begin{aligned}
O\left(\Delta^{\frac{1}{2}}\right): & i k\left(\mathbf{L}_{0}-c_{0} \mathbf{I}\right) \mathbf{q}_{0}=0 \\
O(\Delta): & \frac{\partial}{\partial t} \mathbf{q}_{1}+i k\left(\mathbf{L}_{0}-c_{0} \mathbf{I}\right) \mathbf{q}_{1}+\frac{\partial}{\partial T} \mathbf{q}_{0}=0 \\
O\left(\Delta^{\frac{3}{2}}\right): & \frac{\partial}{\partial t} \mathbf{q}_{2}+i k\left(\mathbf{L}_{0}-c_{0} \mathbf{I}\right) \mathbf{q}_{2}+\frac{\partial}{\partial T} \mathbf{q}_{1} \\
& +i k\left(\mathbf{L}_{2}+\mathbf{N} \overline{q_{C}^{\prime}}\right) \mathbf{q}_{0}=0
\end{aligned}
$$

The eigenvalue $c_{0}$ is the growth rate at the critical point, thus it is a double eigenvalue. We define a vector $\mathbf{r}$ so that

$$
\mathbf{q}_{0}=A(T) \mathbf{r}
$$

thus $\mathbf{r}$ is the eigenvector of $\mathbf{L}_{0} . \mathbf{r}^{\dagger}$ is the left eigenvector and since $c_{0}$ is a double root then $\mathbf{r}^{\dagger} \mathbf{r}=0$. Therefore if we dot the system with $\mathbf{r}^{\dagger}$ then it falls that $\mathbf{q}_{1}$ is also independent of $t$, and then the solvability condition implies that the terms independent of $t$ in the $O\left(\Delta^{\frac{3}{2}}\right)$ equation vanish as well. So that the system becomes

$$
\begin{aligned}
i k\left(\mathbf{L}_{0}-c_{0} \mathbf{I}\right) \mathbf{q}_{1}+\frac{\partial A}{\partial T} \mathbf{r} & =0 \\
\mathbf{r}^{\dagger} \frac{\partial}{\partial T} \mathbf{q}_{1}+i k\left[\mathbf{r}^{\dagger}\left(\mathbf{L}_{2}+\mathbf{N} \overline{q_{C}^{\prime}}\right) \mathbf{r}\right] A & =0
\end{aligned}
$$

The first equation defines $\mathbf{q}_{1}$ so that

$$
\mathbf{q}_{1}=\frac{i \mathbf{s}}{k} \frac{\partial A}{\partial T}
$$

where

$$
\mathbf{s}=\left(\mathbf{L}_{0}-c_{0} \mathbf{I}\right)^{-1} \mathbf{r}
$$

Finally the $O\left(\Delta^{\frac{3}{2}}\right)$ gives an equation for the slow time scale growth of the amplitude of the perturbation

$$
\mathbf{r}^{\dagger} \mathbf{s} \frac{\partial^{2} A}{\partial T^{2}}+k^{2}\left[\mathbf{r}^{\dagger}\left(\mathbf{L}_{2}+\mathbf{N} \overline{q_{C}^{\prime}}\right) \mathbf{r}\right] A=0
$$


All is left is to add the growth of $\overline{q_{C}^{\prime}}$ from the mean equation (B.11). By expanding the same way as in the perturbation equations we find

$$
\begin{gathered}
\left(\frac{\partial}{\partial t}+\Delta^{\frac{1}{2}} \frac{\partial}{\partial T}\right) \overline{q_{C}^{\prime}}+\frac{i k l\left(F_{1}+F_{2}\right)}{\kappa^{2}\left(\kappa^{2}+F_{1}+F_{2}\right)} \\
\cdot\left[\left(\mathbf{q}_{0}(2)+\Delta^{\frac{1}{2}} \mathbf{q}_{1}(2)\right)\left(\mathbf{q}_{0}^{*}(1)+\Delta^{\frac{1}{2}} \mathbf{q}_{1}^{*}(1)\right)-\right. \\
\left.\left(\mathbf{q}_{0}^{*}(2)+\Delta^{\frac{1}{2}} \mathbf{q}_{1}^{*}(2)\right)\left(\mathbf{q}_{0}(1)+\Delta^{\frac{1}{2}} \mathbf{q}_{1}(1)\right)\right]=0
\end{gathered}
$$

where the first components in the vector is the barotropic part and the second is the baroclinic part. Since the lowest order eigenvectors are real (they are at the critical point), the lowest order terms of (B.29) vanish. Then the slow time evolution of the mean is

$$
\begin{array}{r}
\frac{\partial}{\partial T} \overline{q_{C}^{\prime}}+\frac{i k l\left(F_{1}+F_{2}\right)}{\kappa^{2}\left(\kappa^{2}+F_{1}+F_{2}\right)} \\
\cdot\left[\mathbf{q}_{0}(2) \mathbf{q}_{1}^{*}(1)+\mathbf{q}_{1}(2) \mathbf{q}_{0}^{*}(1)-\mathbf{q}_{0}^{*}(2) \mathbf{q}_{1}(1)+\mathbf{q}_{1}^{*}(2) \mathbf{q}_{0}(1)\right]=0
\end{array}
$$

using (B.23) and (B.26) this becomes

$$
\begin{gathered}
\frac{\partial}{\partial T} \overline{q_{C}^{\prime}}+\frac{l\left(F_{1}+F_{2}\right)}{\kappa^{2}\left(\kappa^{2}+F_{1}+F_{2}\right)} \\
\cdot[\mathbf{r}(2) \mathbf{s}(1)-\mathbf{s}(2) \mathbf{r}(1)] \frac{\partial}{\partial T} A^{2}=0
\end{gathered}
$$

Integrating, assuming the correction is initially zero gives

$$
\begin{aligned}
\overline{q_{C}^{\prime}}= & \frac{l\left(F_{1}+F_{2}\right)}{\kappa^{2}\left(\kappa^{2}+F_{1}+F_{2}\right)} \\
& \cdot[\mathbf{r}(2) \mathbf{s}(1)-\mathbf{s}(2) \mathbf{r}(1)]\left(|A|^{2}-|A(0)|^{2}\right)
\end{aligned}
$$

which completely defines the system. Therefore given a basic shear, $F_{1}, F_{2}, \beta_{1}$ and $\beta_{2}$ the weakly non linear stability problem can be solved.

It is clear from (B.28) that if $\mathbf{N}=0$ then (B.28) reduces to an equation with an exponential solution and the growth rate is just the same as in the linear solution (7.33). Therefore we can denote

$$
c_{i}^{2}=\frac{\mathbf{r}^{\dagger} \mathbf{L}_{2} \mathbf{r}}{\mathbf{r}^{\dagger} \mathbf{s}}
$$


and the nonlinear term as

$$
\begin{aligned}
N= & \frac{\mathbf{r}^{\dagger} \mathbf{N} \overline{q_{C}^{\prime}} \mathbf{r}}{\mathbf{r}^{\dagger} \mathbf{s}}=\frac{\mathbf{r}^{\dagger} \mathbf{N r}}{\mathbf{r}^{\dagger} \mathbf{s}} \cdot \frac{l\left(F_{1}+F_{2}\right)}{\kappa^{2}\left(\kappa^{2}+F_{1}+F_{2}\right)} \\
& \cdot[\mathbf{r}(2) \mathbf{s}(1)-\mathbf{s}(2) \mathbf{r}(1)]\left(|A|^{2}-|A(0)|^{2}\right)
\end{aligned}
$$

So finally the equation for the slow time scale amplitude is

$$
\frac{\partial^{2} A}{\partial T^{2}}-k^{2} c_{i}^{2} A+k^{2} N A\left(|A|^{2}-|A(0)|^{2}\right)=0
$$

which gives equation 7.40 . 


\section{Bibliography}

Atkinson, D. H., Ingersoll, A. P., and Seiff, A. (1997). Deep zonal winds on Jupiter: Update of Doppler tracking the Galileo probe from the orbiter. Nature, 388:649650 .

Atkinson, D. H., Pollack, J. B., and Seiff, A. (1996). Galileo Doppler Measurements of the Deep Zonal Winds at Jupiter. Science, 272:842-843.

Atkinson, D. H., Pollack, J. B., and Seiff, A. (1998). The Galileo probe Doppler wind experiment: Measurement of the deep zonal winds on Jupiter. Journal of Geophysical Research, 103:22911-22928.

Aurnou, J. M. and Olson, P. L. (2001). Strong zonal winds from thermal convectionin a rotating spherical shell. Geophysical Research letters, 28(13):2557-2559.

Bagenal, F., Dowling, T. E., and McKinnon, W. B. (2004). Jupiter : the planet, satellites and magnetosphere. Jupiter. The Planet, Satellites and Magnetosphere.

Batchelor, G. K. (1953). The conditions for dynamical similarity of motions of a frictionless perfect-gas atmosphere. Quart. J. R. meteor. Soc., 79:224-235.

Belton, M. J. S., Head, III, J. W., Ingersoll, A. P., Greeley, R., McEwen, A. S., Klaasen, K. P., Senske, D., Pappalardo, R., Collins, G., Vasavada, A. R., Sullivan, R., Simonelli, D., Geissler, P., Carr, M. H., Davies, M. E., Veverka, J., Gierasch, P. J., Banfield, D., Bell, M., Chapman, C. R., Anger, C., Greenberg, R., Neukum, G., Pilcher, C. B., Beebe, R. F., Burns, J. A., Fanale, F., Ip, W., Johnson, T. V., Morrison, D., Moore, J., Orton, G. S., Thomas, P., and West, R. A. (1996). Galileo's First Images of Jupiter and the Galilean Satellites. Science, 274:377-385.

Bosak, T. and Ingersoll, A. P. (2002). Shear Instabilities as a Probe of Jupiter's Atmosphere. Icarus, 158:401-409. 
Boyd, J. P. (2001). Chebyshev and Fourier Spectral Methods. Dover, second edition.

Busse, F. H. (1970). Thermal instabilities in rapidly rotating systems. Journal of Fluid Mechanics, 44:441-460.

Busse, F. H. (1976). A Simple Model of Convection in the Jovian Atmosphere. Icarus, (29):255-260.

Busse, F. H. (1986). Asymptotic theory of convection in a rotating, cylindrical annulus. Journal of Fluid Mechanics, 173:545-556.

Busse, F. H. (1994). Convection Driven Zonal Flows and Vortices in the Major Planets. Chaos, 4(2):123-134.

Busse, F. H. (2002). Convective flows in rapidly rotating spheres and their dynamo action. Physics of Fluids, 14:1301-1314.

Busse, F. H., Hartung, G., Jaletzky, M., and Sommermann, G. (1998). Experiments on thermal convection in rotating systems motivated by planetary problems. Dynamics of Atmospheres and Oceans, 27:161-174.

Busse, F. H. and Hood, L. L. (1982). Differential rotation driven by convection in a rapidly rotating annulus. Geophysical and Astrophysical Fluid Dynamics, 21:59-74.

Campbell, J. K. and Synnott, S. P. (1985). Gravity field of the Jovian system from Pioneer and Voyager tracking data. The Astrophysical Journal, 90:364-372.

Cassini, S. (1672). A Relation of the Return of a Great Permanent Spot in the Planet Jupiter, Observed by Signor Cassini, One of the Royal Parisian Academy of the Sciences. Philosophical Transactions Series I, 7:4039-4042.

Chandrasekhar, S. (1952). Convection Under Terrestrial and Astrophysical Conditions. Publications of the Astronomical Society of the Pacific, 64:98-104.

Chandrasekhar, S. (1961). Hydrodynamic and hydromagnetic stability. International Series of Monographs on Physics, Oxford: Clarendon, 1961.

Cho, J. and Polvani, L. M. (1996). The formation of jets and vortices from freelyevolving shallow water turbulence on the surface of a sphere. Physics of Fluids, 8:1531-1552. 
Cho, J. Y.-K. and Polvani, L. M. (1996). The morphogenesis of bands and zonal winds in the atmospheres on the giant outer planets. Science, 273:335-337.

Christensen, U. R. (2002). Zonal flow driven by strongly supercritical convection in rotating spherical shells. Journal of Fluid Mechanics, 470:115-133.

Collins, G. W., da Silva, L. B., Celliers, P., Gold, D. M., Foord, M. E., Wallace, R. J., Ng, A., Weber, S. V., Budil, K. S., and Cauble, R. (1998). Measurements of the equation of state of deuterium at the fluid insulator-metal transition. Science, 281:1178-1181.

Conrath, B., Flasar, F. M., Hanel, R., Kunde, V., Maguire, W., Pearl, J., Pirraglia, J., Samuelson, R., Cruikshank, D., and Horn, L. (1989). Infrared observations of the Neptunian system. Science, 246:1454-1459.

Conrath, B. J., Flasar, F. M., Pirraglia, J. A., Gierasch, P. J., and Hunt, G. E. (1981). Thermal structure and dynamics of the Jovian atmosphere. II - Visible cloud features. Journal of Geophysical Research, 86:8769-8775.

Deming, D., Reuter, D., Jennings, D., Bjoraker, G., McCabe, G., Fast, K., and Wiedemann, G. (1997). Observations and Analysis of Longitudinal Thermal Waves on Jupiter. Icarus, 126:301-312.

Dowling, T. E. (1993). A relationship between potential vorticity and zonal wind on Jupiter. Journal of Atmospheric Sciences, 50:14-22.

Dowling, T. E. (1995). Estimate of jupiter's deep zonal-wind profile from shoemakerlevy 9 data and arnold's second stability criterion. Icarus, 117:439-442.

Dowling, T. E. and Ingersoll, A. P. (1988). Potential vorticity and layer thickness variations in the flow around jupiter's great red spot and white oval BC. Journal of Atmospheric Sciences, 45(8):1380-1396.

Dowling, T. E. and Ingersoll, A. P. (1989). Jupiter's Great Red Spot as a Shallow Water System. Journal of Atmospheric Sciences, 46(21):3256-3278.

Dowling, T. E. and Spiegel, E. A. (1990). Stellar and Jovian vortices. New York Academy Sciences Annals, 617:190-216. 
Durran, D. R. (1989). Improving the Anelastic Approximation. Journal of Atmospheric Sciences, 46:1453-1461.

Fernando, H. J. S., Chen, R.-R., and Boyer, D. L. (1991). Effects of rotation on convective turbulence. Journal of Fluid Mechanics, 228:513-547.

Flaser, F. M. and Gierasch, P. J. (1986). Mesoscale waves as a probe of Jupiter's deep atmosphere. NASA Conference Publication, 2441:193-194.

Flierl, G. R. (1987). Isolated eddy models in geophysics. Annual Review of Fluid Mechanics, 19:493-530.

Flierl, G. R. (1992). Deep jets and shallow spots. 1992 Summer Study Program in Geophysical Fluid Dynamics, Woods Hole Oceanographic Institution Technical Report No. WHOI-93-24, 1:134-135.

Galperin, B., Sukoriansky, S., and Huang, H.-P. (2001). Universal n-5 spectrum of zonal flows on giant planets. Physics of Fluids, 13:1545-1548.

Galperin, B., Sukoriansky, S., Read, P., Yamazaki, Y., and Wordsworth, R. (2006). Anisotropic turbulence and zonal jets in rotating flows with a beta effect. Nonlinear Processes in Geophysics, 13:83-98.

Gierasch, P. J. (1976). Jovian meteorology - Large-scale moist convection. Icarus, 29:445-454.

Gierasch, P. J., Magalhaes, J. A., and Conrath, B. J. (1986). Zonal mean properties of Jupiter's upper troposphere from Voyager infrared observations. Icarus, 67:456483.

Gilman, P. A. and Glatzmaier, G. A. (1981). Compressible convection in a rotating spherical shell. I - Anelastic equations. II - A linear anelastic model. III - Analytic model for compressible vorticity waves. Astrophysical Journal Supplement Series, $45: 335-388$.

Glatzmaier, G. and Olson, P. (1993). Highly supercritical thermal convection in a rotating spherical shell: Centrifugal vs. radial gravity. Geophysical and Astrophysical Fluid Dynamics, 70:113-136. 
Guillot, T. (2005). The Interiors of giant planets: Models and Outstanding Questions. Annual Review of Earth and Planetary Sciences, 33:493-530.

Guillot, T., Chabrier, G., Morel, P., and Gautier, D. (1994). Nonadiabatic models of Jupiter and Saturn. Icarus, 112:354-367.

Guillot, T. and Morel, P. (1995). CEPAM: a code for modeling the interiors of giant planets. Astronomy and Astrophysics Supplement series, 109:109-123.

Guillot, T., Stevenson, D. J., Hubbard, W. B., and Saumon, D. (2004). The interior of Jupiter, pages 35-57. Jupiter. The Planet, Satellites and Magnetosphere.

Hammel, H. B., Beebe, R. F., Ingersoll, A. P., Orton, G. S., Mills, J. R., Simon, A. A., Chodas, P., Clarke, J. T., de Jong, E., Dowling, T. E., Harrington, J., Huber, L. F., Karkoschka, E., Santori, C. M., Toigo, A., Yeomans, D., and West, R. A. (1995). HST Imaging of Atmospheric Phenomena Created by the Impact of Comet Shoemaker-Levy 9. Science, 267:1288-1296.

Hanel, R., Conrath, B., Herath, L., Kunde, V., and Pirraglia, J. (1981). Albedo, internal heat, and energy balance of Jupiter - Preliminary results of the Voyager infrared investigation. Journal of Geophysical Research, 86:8705-8712.

Hanel, R. A., Conrath, B. J., Kunde, V. G., Pearl, J. C., and Pirraglia, J. A. (1983). Albedo, internal heat flux, and energy balance of Saturn. Icarus, 53:262-285.

Harrington, J., Dowling, T. E., and Baron, R. L. (1996). Jupiter's Tropospheric Thermal Emission. II. Power Spectrum Analysis and Wave Search. Icarus, 124:3244.

Hart, G. (1985). Laboratory experiments on planetary and stellar convection preformed on Spacelab 3. Science, 234:61-234.

Heimpel, M. and Aurnou, J. (2007). Turbulent convection in rapidly rotating spherical shells: A model for equatorial and high latitude jets on Jupiter and Saturn. Icarus, $187: 540-557$.

Heimpel, M., Aurnou, J., and Wicht, J. (2005). Simulation of equatorial and highlatitude jets on Jupiter in a deep convection model. Nature, 438:193-196. 
Held, I. M. and Hou, A. Y. (1980). Nonlinear Axially Symmetric Circulations in a Nearly Inviscid Atmosphere. Journal of Atmospheric Sciences, 37:515-533.

Huang, H., Galperin, B., and Sukoriansky, S. (2001). Generation of mean flows and jets on a beta plane and over topography. Physics of Fluids, 13:225-240.

Huang, H. P. and Robinson, W. A. (1998). Two-Dimentional Turbulence and Persistent Jets in a Global Barotropic Model. Journal of Atmospheric Science, 55:611632.

Hubbard, W. B. (1968). Thermal structure of Jupiter. The Astrophysical Journal, 152:745-754.

Hubbard, W. B. (1999). NOTE: Gravitational Signature of Jupiter's Deep Zonal Flows. Icarus, 137:357-359.

Iacono, R., Struglia, M. V., and Ronchi, C. (1999a). Spontaneous formation of equatorial jets in freely decaying shallow water turbulence. Physics of Fluids, 11:12721274 .

Iacono, R., Struglia, M. V., Ronchi, C., and Nicastro, S. (1999b). High-resolution simulations of freely decaying shallow-water turbulence on a rotating sphere. Nuovo Cimento C Geophysics Space Physics C, 22:813-+.

Ingersoll, A. P. (1976). Pioneer 10 and 11 observations and the dynamics of Jupiter's atmosphere. Icarus, 29:245-252.

Ingersoll, A. P. (1990). Atmospheric Dynamics of the Outer Planets. Science, 248.

Ingersoll, A. P. (2005). Boussinesq and Anelastic Approximations Revisited: Potential Energy Release during Thermobaric Instability. Journal of Physical Oceanography, 35:1359-1369.

Ingersoll, A. P., Beebe, R. F., Conrath, B. J., and Hunt, G. E. (1984). Structure and dynamics of Saturn's atmosphere, pages 195-238. Saturn.

Ingersoll, A. P. and Cuong, P. G. (1981). Numerical model of long-lived Jovian vortices. Journal of Atmospheric Sciences, 38:2067-2076. 
Ingersoll, A. P. and Cuzzi, J. N. (1969). Dynamics of Jupiter's cloud bands. Journal of Atmospheric Sciences, 26:981-985.

Ingersoll, A. P. and Pollard, D. (1982). Motion in the Interiors and Atmospheres of Jupiter and Saturn: Scale Analysis, Anelastic Equations, Barotropic Stability Criterion. Icarus, 52:62-80.

Ingersoll, A. P. and Porco, C. C. (1978). Solar heating and internal heat flow on Jupiter. Icarus, 35:27-43.

Ioannou, P. and Lindzen, R. (1994). Gravitational tides in the outer planets 3.atmospheric response and the mean flow acceleration. The Astrophysical Journal, 424(2):1005-1013.

Ioannou, P. and Lindzen, R. S. (1993a). Gravitational tides in the outer planets 1.implications of classical tidal theory. The Astrophysical Journal, 406(1):252-265.

Ioannou, P. and Lindzen, R. S. (1993b). Gravitational tides in the outer planets 2. interior calculations and estimation of the tidal dissipation factor. The Astrophysical Journal, 406(1):266-278.

Irwin, P. G. J. (2003). Giant planets of our solar system : atmospheres compositions, and structure. Giant planets of our solar system : atmospheres, compositions, and structure, by P.G.J. Irwin. Springer Praxis books in geophysical sciences. Berlin: Springer, 2003.

Jones, C. A., Soward, A. M., and Mussa, A. I. (2000). The onset of thermal convection in a rapidly rotating sphere. Journal of Fluid Mechanics, 405:157-179.

Kaspi, Y. and Flierl, G. R. (2007). Formation of Jets by Baroclinic Instability on Gas Planet Atmospheres. The Journal of the Atmospheric Sciences, 64:3177-3194.

Kippenhahn, R. and Weigert, A. (1990). Stellar Structure and Evolution. Stellar Structure and Evolution, XVI, 468 pp. 192 figs.. Springer-Verlag Berlin Heidelberg New York. Also Astronomy and Astrophysics Library.

Kirk, R. L. and Stevenson, D. J. (1987). Hydromagnetic constraints on deep zonal flow in the giant planets. The Astrophysical Journal, 316:836-846. 
Knudson, M. D., Hanson, D. L., Bailey, J. E., Hall, C. A., Asay, J. R., and Anderson, W. W. (2001). Equation of State Measurements in Liquid Deuterium to 70 GPa. Physical Review Letters, 87(22):225501-+.

Kuo, H. L. (1949). Dynamic instability of two-dimensional nondivergent flow in a barotropic atmosphere. J. Meteor, 6:105-122.

Lee, S. (2004). Baroclinic multiple jets on a sphere. Journal of Atmospheric Science, 62:2484-2498.

Leovy, C. B., Friedson, A. J., and Orton, G. S. (1991). The quasiquadrennial oscillation of Jupiter's equatorial stratosphere. Nature, 354:380-382.

Lian, Y., Showman, A. P., and Gierasch, P. J. (2006). Three-Dimensional Nonlinear Simulations of Jets on Giant Planets. To be submitted to Icarus.

Lilly, J. M., Rhines, P. B., Visbeck, M., Davis, R., Lazier, J. R. N., Schott, F., and Farmer, D. (1999). Observing Deep Convection in the Labrador Sea during Winter 1994/95. Journal of Physical Oceanography, 29:2065-2098.

Limaye, S. S. (1986). Jupiter: New estimates of mean zonal flow at the cloud level. In Allison, M. and Travis, L. D., editors, Jovian Atmospheres, pages 124-128.

Lindal, G. F., Wood, G. E., Levy, G. S., Anderson, J. D., Sweetnam, D. N., Hotz, H. B., Buckles, B. J., Holmes, D. P., Doms, P. E., Eshleman, V. R., Tyler, G. L., and Croft, T. A. (1981). The atmosphere of Jupiter - an analysis of the Voyager radio occultation measurements. Journal of Geophysical research, 86:8721-8727.

Lindzen, R. S. (1977). Some Aspects of Convection in Meteorology. Lecture Notes in Physics, Berlin Springer Verlag, 71:128-+.

Lindzen, R. S. (1991). Some remarks on the dynamics of the jovian atmospheres. Geophysical and Astrophysical Fluid Dynamics, 58:123-131.

Liu, J. (2006). Interaction of magnetic field and flow in the outer shells of giant planets. PhD thesis, California Institute of Technology.

Magalhaes, J. A., Weir, A. L., Conrath, B. J., Gierasch, P. J., and Leroy, S. S. (1989). Slowly moving thermal features on Jupiter. Nature, 337:444-447. 
Manifori, A. J. and Young, W. R. (1999). Slow Evolution of Zonal Jets on a Beta Plane. Journal of the Atmospheric Sciences, 56:784-800.

Manneville, J. B. and Olson, P. (1996). Banded convection in rotating fluid spheres and the circulation of the jovian atmosphere. Icarus, 122:242-250.

Marcus, P. S. (1988). Numerical simulation of Jupiter's Great Red SPOT. Nature, 331:693-696.

Marcus, P. S. (1990). Vortex dynamics in a shearing zonal flow. Journal of Fluid Mechanics, 215:393-430.

Marshall, J. and Schott, F. (1999). Open-ocean convection: Observations, theory, and models. Reviews of Geophysics, 37:1-64.

Mehta, A. V. (1998). The role of vortices in the formation of the solar system. $\mathrm{PhD}$ thesis, M.I.T.

Morse, P. M. and Feshbach, H. (1953). Methods of theoretical physics. International Series in Pure and Applied Physics, New York: McGraw-Hill, 1953.

Nellis, W. J., Holmes, N. C., Mitchell, A. C., Governo, G. K., Ross, M., Young, D. A., and Trainor, R. J. (1984). Shock compression of liquid helium to $56 \mathrm{GPa}$ (560 kbar). Physical Review Letters, 53:1248-1251.

Ogura, Y. and Phillips, N. A. (1962). Scale Analysis of Deep and Shallow Convection in the Atmosphere. Journal of the Atmospheric Sciences, 19:173-179.

Ortiz, J. L., Orton, G. S., Friedson, A. J., Stewart, S. T., Fisher, B. M., and Spencer, J. R. (1998). Evolution and persistence of $5-\mu \mathrm{m}$ hot spots at the Galileo probe entry latitude. Journal of Geophysical Research, 103:23051-23069.

Orton, G. S., Friedson, A. J., Caldwell, J., Hammel, H. B., Baines, K. H., Bergstralh, J. T., Martin, T. Z., Malcom, M. E., West, R. A., Golisch, W. F., Griep, D. M., Kaminski, C. D., Tokunaga, A. T., Baron, R., and Shure, M. (1991). Thermal maps of Jupiter - Spatial organization and time dependence of stratospheric temperatures, 1980 to 1990. Science, 252:537-542. 
Orton, G. S., Friedson, A. J., Yanamandra-Fisher, P. A., Caldwell, J., Hammel, H. B., Baines, K. H., Bergstralh, J. T., Martin, T. Z., West, R. A., Veeder, Jr., G. J., Lynch, D. K., Russell, R., Malcom, M. E., Golisch, W. F., Griep, D. M., Kaminski, C. D., Tokunaga, A. T., Herbst, T., and Shure, M. (1994). Spatial Organization and Time Dependence of Jupiter's Tropospheric Temperatures, 1980-1993. Science, 265:625-631.

Panetta, R. L. (1993). Zonal jets in wide baroclinically unstable regions: Persistence and scale selection. Journal of the Atmospheric Sciences, 50(14):2073-2106.

Pearl, J. C. and Conrath, B. J. (1991). The albedo, effective temperature, and energy balance of Neptune, as determined from Voyager data. Journal of Geophysical research, 96:18921-+.

Pearl, J. C., Conrath, B. J., Hanel, R. A., and Pirraglia, J. A. (1990). The albedo, effective temperature, and energy balance of Uranus, as determined from Voyager IRIS data. Icarus, 84:12-28.

Pedlosky, J. (1970). Finite-amplitude baroclinic wave. Journal of the Atmospheric Science, 27:15-30.

Pedlosky, J. (1987). Geophysical Fluid Dynamics. Spinger.

Phillips, N. A. (1954). Energy transformations and meridional circulations associated with simple baroclinic waves in a two level quasi-geostrophic model. Telus, 6:273286.

Pirraglia, J. A., Conrath, B. J., Allison, M. D., and Gierasch, P. J. (1981). Thermal structure and dynamics of Saturn and Jupiter. Nature, 292:677-679.

Porco, C. C., Baker, E., Barbara, J., Beurle, K., Brahic, A., Burns, J. A., Charnoz, S., Cooper, N., Dawson, D. D., Del Genio, A. D., Denk, T., Dones, L., Dyudina, U., Evans, M. W., Giese, B., Grazier, K., Helfenstein, P., Ingersoll, A. P., Jacobson, R. A., Johnson, T. V., McEwen, A., Murray, C. D., Neukum, G., Owen, W. M., Perry, J., Roatsch, T., Spitale, J., Squyres, S., Thomas, P., Tiscareno, M., Turtle, E., Vasavada, A. R., Veverka, J., Wagner, R., and West, R. (2005). Cassini Imaging Science: Initial Results on Saturn's Atmosphere. Science, 307:1243-1247. 
Porco, C. C., West, R. A., McEwen, A., Del Genio, A. D., Ingersoll, A. P., Thomas, P., Squyres, S., Dones, L., Murray, C. D., Johnson, T. V., Burns, J. A., Brahic, A., Neukum, G., Veverka, J., Barbara, J. M., Denk, T., Evans, M., Ferrier, J. J., Geissler, P., Helfenstein, P., Roatsch, T., Throop, H., Tiscareno, M., and Vasavada, A. R. (2003). Cassini Imaging of Jupiter's Atmosphere, Satellites and Rings. Science, 299:1541-1547.

Reuter, D. C., Simon-Miller, A. A., Lunsford, A., Baines, K. H., Cheng, A. F., Jennings, D. E., Olkin, C. B., Spencer, J. R., Stern, S. A., Weaver, H. A., and Young, L. A. (2007). Jupiter Cloud Composition, Stratification, Convection, and Wave Motion: A View from New Horizons. Science, 318:223-.

Rhines, P. B. (1975). Waves and turbulence on a beta plane. Journal of Fluid Mechanics, 69:417-443.

Rhines, P. B. (1979). Geostrophic Turbulence. Annual Reviews of Fluid Mechanics, 11:401-441.

Roberts, P. H. (1968). On the thermal instability of a rotating-fluid sphere containing heat sources. Royal Society of London Philosophical Transactions Series A, 263:93117.

Robinson, A. R. and McWilliams, J. (1974). The baroclinic instability of the open ocean. Journal of physical oceanography, 14:281-294.

Salpeter, E. E. (1973). On Convection and Gravitational Layering in Jupiter and in Stars of Low Mass. The Astrophysical Journal, 181:L83+.

Sanchez-Lavega, A., Hueso, R., Lecacheux, J., Colas, F., Rojas, J. F., Gomez, J. M., Miyazaki, I., and Parker, D. (1998). Dynamics and Interaction between a LargeScale Vortex and the Great Red SPOT in Jupiter. Icarus, 136:14-26.

Sánchez-Lavega, A., Orton, G. S., Hueso, R., García-Melendo, E., Pérez-Hoyos, S., Simon-Miller, A., Rojas, J. F., Gómez, J. M., Yanamandra-Fisher, P., Fletcher, L., Joels, J., Kemerer, J., Hora, J., Karkoschka, E., de Pater, I., Wong, M. H., Marcus, P. S., Pinilla-Alonso, N., Carvalho, F., Go, C., Parker, D., Salway, M., Valimberti, M., Wesley, A., and Pujic, Z. (2008). Depth of a strong jovian jet from a planetary-scale disturbance driven by storms. Nature, 451:437-440. 
Sanchez-Lavega, A., Orton, G. S., Morales, R., Lecacheux, J., Colas, F., Fisher, B., Fukumura-Sawada, P., Golisch, W., Griep, D., Kaminski, C., Baines, K., Rages, K., and West, R. (2001). NOTE: The Merger of Two Giant Anticyclones in the Atmosphere of Jupiter. Icarus, 149:491-495.

Sánchez-Lavega, A., Pérez-Hoyos, S., Rojas, J. F., Hueso, R., and French, R. G. (2003). A strong decrease in Saturn's equatorial jet at cloud level. Nature, 423:623625.

Saumon, D., Chabrier, G., and van Horn, H. M. (1995). An Equation of State for Low-Mass Stars and Giant Planets. The astrophysical journal, 99:713-+.

Schneider, T. (2006). The General Circulation of the Atmospherea. Annual Review of Earth and Planetary Sciences, 34:655-688.

Seiff, A., Kirk, D. B., Knight, T. C. D., Mihalov, J. D., Blanchard, R. C., Young, R. E., Schubert, G., von Zahn, U., Lehmacher, G., Milos, F. S., and Wang, J. (1996). Structure of the Atmosphere of Jupiter: Galileo Probe Measurements. Science, 272:844-845.

Seiff, A., Kirk, D. B., Knight, T. C. D., Young, L. A., Milos, F. S., Venkatapathy, E., Mihalov, J. D., Blanchard, R. C., Young, R. E., and Schubert, G. (1997). Thermal structure of Jupiter's upper atmosphere derived from the Galileo probe. Science, 276:102-104.

Sheremet, V. A. (2004). Laboratory experiments with tilted convective plumes on a centrifuge: a finite angle between the buoyancy force and the axis of rotation. Journal of Fluid Mechanics, 506:217-244.

Showman, A. P. (2004). Effects of moist convection on the large-scale circulation of Jupiter and Saturn. In Bulletin of the American Astronomical Society, volume 36 of Bulletin of the American Astronomical Society, pages 1135-+.

Showman, A. P. (2007). Numerical simulations of forced shallow-water turbulence: effects of moist convection on the large-scale circulation of Jupiter and Saturn. The Journal of Atmos. Sci., 64:3132-3157.

Showman, A. P. and Dowling, T. E. (2000). Nonlinear Simulations of Jupiter's 5Micron Hot Spots. Science, 289:1737-1740. 
Showman, A. P., Gierasch, P. J., and Lian, Y. (2006). Deep zonal winds can result from shallow driving in a giant-planet atmosphere. Icarus, 182:513-526.

Simon, A. A. (1999). The Structure and Temporal Stability of Jupiter's Zonal Winds: A Study of the North Tropical Region. Icarus, 141:29-39.

Simon, A. A., Beebe, R. F., Gierasch, P. J., Vasavada, A. R., and Belton, M. J. S. (1998). Global Context of the Galileo-E6 Observations of Jupiter's White Ovals. Icarus, 135:220-229.

Simon-Miller, A. A., Gierasch, P. J., Beebe, R. F., Conrath, B., Flasar, F. M., Achterberg, R. K., and the Cassini CIRS Team (2002). New Observational Results Concerning Jupiter's Great Red Spot. Icarus, 158:249-266.

Smith, B. A., Soderblom, L., Batson, R., Bridges, P., Inge, J., Masursky, H., Shoemaker, E., Beebe, R., Boyce, J., Briggs, G., Bunker, A., Collins, S. A., Hansen, C. J., Johnson, T. V., Mitchell, J. L., Terrile, R. J., Cook, A. F., Cuzzi, J., Pollack, J. B., Danielson, G. E., Ingersoll, A., Davies, M. E., Hunt, G. E., Morrison, D., Owen, T., Sagan, C., Veverka, J., Strom, R., and Suomi, V. E. (1982). A new look at the Saturn system: The Voyager 2 images. Science, 215:505-537.

Smith, B. A., Soderblom, L. A., Beebe, R., Bliss, D., Brown, R. H., Collins, S. A., Boyce, J. M., Briggs, G. A., Brahic, A., Cuzzi, J. N., and Morrison, D. (1986). Voyager 2 in the Uranian system - Imaging science results. Science, 233:43-64.

Smith, K. S. (2003). A Local Model for Planetary Atmospheres Forced by Small-Scale Convection. Journal of Atmospheric Science, 61:1420-1433.

Sprague, M., Julien, K., Knobloch, E., and Werne, J. (2006). Numerical simulation of an asymptotically reduced system for rotationally constrained convection. Journal of Fluid Mechanics, 551:141-174.

Stamp, A. P. and Dowling, T. E. (1993). Jupiter's winds and Arnol'd's second stability theorem: Slowly moving waves and neutral stability. Journal of Geophysical Research, 98:18847-18855.

Steinsaltz, D. (1987). Instability of Baroclinic Waves with Bottom Slope. Journal of Physical Oceanography, 17(12):2343-2350. 
Stevenson, D. J. (1991). The search for brown dwarfs. Annual Review of Astronomy and Astrophysics, 29:163-193.

Stevenson, D. J. and Salpeter, E. E. (1976). Interior models of Jupiter. In Gehrels, T., editor, IAU Colloq. 30: Jupiter: Studies of the Interior, Atmosp here, Magnetosphere and Satellites, pages 85-112.

Sun, Z.-P., Schubert, G., and Glatzmaier, G. A. (1993). Banded surface flow maintained by convection in a model of the rapidly rotating giant planets. Science, 260:661-664.

Trigo-Rodriguez, J. M., Sánchez-Lavega, A., Gómez, J. M., Lecacheux, J., Colas, F., and Miyazaki, I. (2000). The 90-day oscillations of Jupiter's Great Red Spot revisited. Planatary and Space Science, 48:331-339.

Vallis, G. K. (2006). Atmospheric and Oceanic Fluid Dynamics. Atmospheric and Oceanic Fluid Dynamics, by Geoffrey K. Vallis, pp. 770. Cambridge University Press, November 2006. ISBN-10: 0521849691. ISBN-13: 9780521849692.

Vallis, G. K. and Maltrud, M. E. (1993). Generation of mean flows and jets on a beta plane and over topography. Journal of Physical Oceanography, 23:1346-1362.

Vasavada, A. R., Ingersoll, A. P., Banfield, D., Bell, M., Gierasch, P. J., Belton, M. J. S., Orton, G. S., Klaasen, K. P., Dejong, E., Breneman, H. H., Jones, T. J., Kaufman, J. M., Magee, K. P., and Senske, D. A. (1998). Galileo Imaging of Jupiter's Atmosphere: The Great Red Spot, Equatorial Region, and White Ovals. Icarus, 135:265-275.

Vasavada, A. R. and Showman, A. P. (2005). Jovian atmospheric dynamics: an update after Galileo and Cassini. Reports of Progress in Physics, 68:1935-1996.

Veronis, G. (1959). Cellular convection with finite amplitude in a rotating fluid. Journal of Fluid Mechanics, 5:401-435.

Weir, S. T., Mitchell, A. C., and Nellis, W. J. (1996). Metallization of Fluid Molecular Hydrogen at 140 GPa (1.4 Mbar). Physical Review Letters, 76:1860-1863.

Williams, G. P. (1978). Planetary Circulations: 1. Barotropic representation of the Jovian and Terrestrial Turbulence. Journal of the Atmospheric Sciences, 35. 
Williams, G. P. (1979). Planetary Circulations: 2. The Jovian Quasi-Geostrophic Regime. Journal of the Atmospheric Sciences, 36.

Williams, G. P. (2003). Jovian dynamics. part 3: Multiple, migrating and equatorial jets. Journal of the Atmospheric Sciences, 60:1270-1296.

Yano, J. (1994). A critical review on the dynamics of Jovian atmospheres. Chaos, $4: 287-297$.

Yano, J.-I. (1992). Asymptotic theory of thermal convection in rapidly rotating systems. Journal of Fluid Mechanics, 243:103-131.

Yano, J. I. and Flierl, G. R. (1994). Jupiter's Great Red Spot: compactness condition and stability. Annales Geophysicae, 12:1-18.

Yano, Y. I. (2005). Origins of atmospheric zonal winds. Nature, 421:36.

Youssef, A. and Marcus, P. S. (2003). The dynamics of jovian white ovals from formation to merger. Icarus, 162:74-93.

Zhang, K. (1992). Spiralling columnar convection in rapidly rotating spherical fluid shells. Journal of Fluid Mechanics, 236:535-556.

Zhang, K. (1994). On coupling between the Poincare equation and the heat equation. Journal of Fluid Mechanics, 268:211-229.

Zhang, K. and Busse, F. H. (1987). On the onset of convection in rotating spherical shells. Geophysical and Astrophysical Fluid Dynamics, 39:119-147.

Zhang, K. and Schubert, G. (1996). Penetrative Convection and Zonal Flow on Jupiter. Science, 273:941-943.

Zhang, K. and Schubert, G. (1997). Linear Penetrative Spherical Rotating Convection. Journal of the Atmospheric Sciences, 54(21):2509-2518. 